Astronomy and Astrophysics Review manuscript No.

(will be inserted by the editor)

\author{
Joachim Puls · Jorick S. Vink · \\ Francisco Najarro
}

\title{
Mass loss from hot massive stars
}

Received: date

\begin{abstract}
Mass loss is a key process in the evolution of massive stars, and must be understood quantitatively if it is to be successfully included in broader astrophysical applications such as galactic and cosmic evolution and ionization. In this review, we discuss various aspects of radiation driven mass loss, both from the theoretical and the observational side. We focus on developments in the past decade, concentrating on the winds from OB-stars, with some excursions to the winds from Luminous Blue Variables (including super-Eddington, continuum-driven winds), winds from Wolf-Rayet stars, A-supergiants and Central Stars of Planetary Nebulae. After recapitulating the 1-D, stationary standard model of line-driven winds, extensions accounting for rotation and magnetic fields are discussed. Stationary wind models are presented that provide theoretical predictions for the mass-loss rates as a function of spectral type, metallicity, and the proximity to the Eddington limit. The relevance of the so-called bi-stability jump is outlined. We summarize diagnostical methods to infer wind properties from observations, and compare the results from corresponding campaigns (including the VLT-FLAMES survey of massive stars) with theoretical predictions, featuring the mass loss-metallicity dependence. Subsequently, we concentrate on two urgent problems, weak winds and wind-clumping, that have been identified from various diagnostics and that challenge our present understanding of radiation driven winds. We discuss the problems of "measuring" mass-loss rates from weak winds and the potential of the NIR $\mathrm{Br}_{\alpha}$-line as a tool to enable a more pre-
\end{abstract}

Joachim Puls

Universitätssternwarte München, Scheinerstr. 1, D-81679 München, Germany

E-mail: uh101aw@usm.uni-muenchen.de

Jorick S. Vink

Armagh Observatory, College Hill, Armagh BT61 9DG, Northern Ireland

E-mail: jsv@arm.ac.uk

Francisco Najarro

Instituto de Estructura de la Materia, CSIC, Serrano 121, 28006 Madrid, Spain

E-mail: najarro@damir.iem.csic.es 
cise quantification, and comment on physical explanations for mass-loss rates that are much lower than predicted by the standard model. Wind-clumping, conventionally interpreted as the consequence of a strong instability inherent to radiative line-driving, has severe implications for the interpretation of observational diagnostics, since derived mass-loss rates are usually overestimated when clumping is present but ignored in the analyses. Depending on the specific diagnostics, such overestimates can amount to factors of 2 to 10 , and we describe ongoing attempts to allow for more uniform results. We point out that independent arguments from stellar evolution favor a moderate reduction of present-day mass-loss rates. We also consider larger scale wind structure, interpreted in terms of co-rotating interacting regions, and complete this review with a discussion of recent progress on the X-ray line emission from massive stars. Such emission is thought to originate both from magnetically confined winds and from non-magnetic winds, in the latter case related to the line-driven instability and/or clump-clump collisions. We highlight as to how far the analysis of such X-ray line emission can give further clues regarding an adequate description of wind clumping.

Keywords hydrodynamics $\cdot$ stars: atmospheres $\cdot$ stars: early-type $\cdot$ stars: mass loss $\cdot$ stars: winds, outflows

\section{Introduction}

Within the last decade, our understanding of the physics of massive stars has significantly improved. It has been realized that massive stars are critical agents in galactic evolution, during both the present epoch as well as in the early Universe, and they are regarded as "cosmic engines" (Bresolin et al 2008). For instance, a population of very massive, First Stars (for a recent review, see Johnson et al 2008) is thought to play a dominant role in the reionization of the Universe and its first enrichment with metals. Already with the next generation of telescopes, the integrated light from these First Generations of Stars might become observable in the near-infrared (NIR), by means of $\mathrm{Ly}_{\alpha}$ (Barton et al 2004) and He II $\lambda 1640$ (Kudritzki and The GSMT Science Working Group 2003) emission from the surrounding interstellar medium, if the corresponding initial mass function (IMF) is indeed top-heavy (Abel et al 2000, Bromm et al 2001). A proper knowledge of the mass-loss mechanisms during such early stages is crucial to understand the interplay of these First Stars with their environments. Another highlight concerns the long gamma-ray bursters (GRBs), which are likely the result of the terminal collapse of massive stars at low metallicity. GRBs may also become important tracers of the star formation history of the Universe at high redshift. Again, mass loss plays a dominant role in the evolution of angular-momentum loss, thus controlling whether the star will become such a burster.

There are three factors which have contributed to the aforementioned progress: (i) new observational facilities such as ground-based 10-m class telescopes equipped with multi-object spectrographs, and space-born observatories with spectroscopic capabilities in the ultraviolet (HST, FUSE, GALEX), infrared (SPITZER), X-ray (XMM-NEWTON, CHANDRA), and in the $\gamma$-ray (HETE-2, SWIFT) domains; (ii) the development of simulations of complex physical processes in the interior 
and outer envelopes of massive stars, such as rotation and magnetic fields; and (iii) the advancement of model atmospheres and radiative transfer techniques.

Modeling the atmospheres of hot stars is a tremendous challenge due to severe departures from Local Thermodynamic Equilibrium (LTE) because of the intense radiation, low densities, and the presence of supersonic velocity fields initiated by the transfer of momentum from the stellar radiation field to the atmospheric plasma. Radiation-driven winds are fundamentally important, in providing energy (kinetic and ionizing radiation) and momentum input into the ISM, in creating wind-blown bubbles and circumstellar shells, and in triggering star formation. They can affect stellar evolution by modifying evolutionary timescales, surface abundances, and stellar luminosities. The mass loss does not only influence the stellar evolution but also the atmospheric structure, and winds need to be properly modeled to derive the correct stellar parameters by means of quantitative spectroscopy.

The last review on radiation-driven winds from hot stars was given by Kudritzki and Puls (2000) who concentrated on the derivation and calibration of the so-called windmomentum luminosity relation (WLR) and its potential application as an extragalactic distance indicator. Thereafter, efficient methods have been developed that allow for theoretical predictions of mass-loss rates as a function of stellar parameters in the complete upper HRD (Vink et al 2000, 2001), and these can be used to check our standard picture of radiation-driven winds through comparison with empirical results. Owing to the enormous improvements in observational capabilities and diagnostic methods, a number of puzzling phenomena have meanwhile emerged, and this has resulted in exciting speculations and theoretical developments which are by no means settled. To name two of the most prominent, there is the so-called weak-wind problem which indicates that the mass-loss rates from late O-/early B-type dwarfs might be a factor of 10 to 100 lower than theoretically expected, challenging our understanding of radiation-driven winds. Second, there is the issue of wind-clumping which refers to small-scale density inhomogeneities distributed across the wind. Because clumping "contaminates" almost all our mass-loss sensitive diagnostics, it might result in severe down-sizing of previously derived mass-loss rates, and consequently impact significantly our understanding of stellar and galactic evolution.

It is the goal of this review to provide an overview of these developments, to outline their implications, and to indicate possible directions for future work. Because of the breadth of the topic and its complexity, we cannot cover all aspects. Instead, we primarily focus our review on the winds from single "normal" hot massive stars in well-established evolutionary stages such as OB-dwarfs, giants, and supergiants, and on some of the most relevant aspects of the winds from Luminous Blue Variables (LBVs) and Wolf-Rayet (WR) stars (detailed in a recent review by Crowther 2007). We also consider the winds from Central Stars of Planetary Nebulae (CSPN) which are thought to obey similar physical rules as their massive O-star counterparts. For a review on physical processes related to wind-wind collisions in binary systems, we refer to De Becker (2007).

This review is organized in such a way that most chapters can be read independently from each other. Sect. 2.1 provides the basic line-driven wind theory in terms of a 1-D, stationary model with statistically distributed lines. In Sect. 2.2 this "standard model" is extended by accounting for the interaction with rotation 
and magnetic fields, which are not only important when the field strengths are large, as it is the case for few "normal' 11 OB stars, but also when the wind density is quite low and relatively modest fields are present.

In Sect. 3, we compare and contrast the various stationary wind models providing theoretical predictions for the mass-loss rates as a function of spectral type, metallicity, and the proximity to the Eddington limit. We focus on predictions obtained by Monte Carlo methods for stars in various evolutionary phases (OBA, LBV, WR) and for various chemical mixtures. These prescriptions are used in most modern evolutionary calculations and are generally referred to when observational findings are compared to theory. An issue of particular relevance is the so-called bi-stability jump. Objects below a specific effective temperature (around $25 \mathrm{kK}, \mathrm{kK}=$ kilo Kelvin) should have systematically larger mass-loss rates and lower terminal wind velocities than objects above this jump temperature, due to an abrupt change in the ionization equilibrium of iron, the most important element with respect to line-driving in the inner wind.

After a brief discussion of various wind-diagnostics and their pros and cons, recent observational results and their comparisons with theoretical predictions are summarized in Sect. 4 We first demonstrate how the continuing development of NLTE model atmospheres has led to severe parameter changes due to the inclusion of metal-line and wind-blanketing. Most affected is the effective temperature scale of Galactic OB-stars, with the new temperatures significantly below the older ones. We subsequently present observationally derived wind-parameters for OBA-stars, LBVs, and WRs, and we discuss their implications. Special emphasis is given to the VLT-FLAMES survey of massive stars in the Galaxy and the Magellanic Clouds (Evans et al 2005, 2006) which allowed for a derivation of stellar and wind parameters of a large sample of objects, enabling a detailed empirical study of the mass loss-metallicity dependence. We also cover topics such as the winds from BA-supergiants beyond the Magellanic Clouds, the winds from CSPN, and we end with an excursion to super-Eddington, continuum-driven winds which might be relevant for the giant eruptions of LBVs.

At first glance, Sect. 4 might give the impression that theory and observations largely agree and that only a few items remain to be clarified before hot-star winds can be regarded as "understood". Indeed this was a widely held belief at the end of the 1990's. In the meantime, however, the issues of wind clumping and weak winds (see above) have now emerged as urgent.

In Sect. 5 we specify the "weak wind problem" and discuss various physical processes which might be responsible for the apparent dilemma. We demonstrate the challenges for conventional mass-loss diagnostics that become rather insensitive for very low mass-loss rates. As an alternative diagnostic, we discuss the potential of the NIR $\mathrm{Br}_{\alpha}$-line as a tool to enable more precise measurements and that might allow for a quantification of the mass-loss "deficit" with respect to theoretical predictions.

Sect. 6 is devoted to another central topic of current activity in stellar wind research, comprising various aspects of wind-structure and time-dependence neglected in the standard model. After a summary of observational findings, we provide theoretical arguments which show that line-driven winds are subject to a strong instability inherent to the line acceleration itself. This line-driven (or

\footnotetext{
1 as opposed to the chemically peculiar Ap/Bp-stars.
} 
de-shadowing) instability has been proposed to be the origin of wind-clumping, triggering the formation of small-scale structure. In Sect. 6.3, we discuss this in detail. Clumping has severe implications for the interpretation of observed lineprofiles, particularly with respect to the derived mass-loss rates. Conventional recombination-based $\rho^{2}$-processes such as $\mathrm{H}_{\alpha}$, IR, and radio-excess become very sensitive to clumping, resulting in overestimated mass-loss rates when clumping is present but has been neglected in the analyses. We discuss various diagnostic methods to investigate the clumping properties of stellar winds and to derive "true" mass-loss rates. Corresponding results disagree with respect to the estimated degree of mass-loss reduction, ranging from factors in between 2 and 10, and even more. Potential problems with these diagnostics have been identified by different research groups and are likely related to the porosity of a clumped wind medium and the corresponding velocity space, or vorosity. We finish the section with some independent insights from stellar evolution, which suggest that the reduction of the mass-loss rates is more likely to be moderate.

Turning to larger-scale structure, Sect.6.4 covers the discrete absorption components (DACs) and modulation features, as well as corresponding time-dependent models of co-rotating interaction zones (CIRs) and co-rotating, azimuthally extended structures which might be responsible for these other structural phenomena.

Since the first X-ray measurements of massive stars became available, it was clear that they are strong X-ray emitters. In Sect. 6.5, we review the status of the X-ray wind line emission, as observed by XMM-NEWTON and CHANDRA. This emission has been interpreted to either result from magnetically confined winds or to result from shocks embedded in the wind, likely related to the linedriven instability or clump-clump collisions. Recent diagnostics has enabled to "measure" the onset of this emission, and current analyses utilize the line emission as a tool to deduce the degree of wind-clumping, its radial distribution, and the shape of the clumps. We report on corresponding results and implications.

In Sect. 7 we summarize the most important achievements obtained during the last decade of wind research and provide some future prospects.

\section{Line driven winds of hot stars - theoretical background}

As each photon carries momentum, $h v / c$, it was speculated as early as in the 1920s (e.g., Milne 1926) that radiative acceleration by spectral lines might be capable of ejecting metal ions from stellar atmospheres. However, it was not until UV balloon flights in the late 1960s that the theory of radiation line driving became the leading theory for the steady outflows from hot luminous OB stars. Lucy and Solomon (1970) and Castor et al (1975) realized that if the acceleration exerted on the metal ions could be shared with the more abundant hydrogen and

helium species in the plasma, it could result in a significant mass-loss rate $\dot{M}$, of the order of $10^{-6} M_{\odot} \mathrm{yr}^{-1}$, which might affect the evolution of massive stars in a substantial manner. 


\subsection{The standard model (1-D, stationary, smooth)}

Momentum transfer by line scattering. In the following, we will concentrate on those lines that contribute to most of the total acceleration, i.e., resonance $=$ scattering lines from ionic ground states $(\mathrm{C}, \mathrm{N}, \mathrm{O}, \ldots)$ or low-lying meta-stable levels (iron group elements). The principal concept of momentum transfer by linescattering is that the initial photon originates from a particular direction in the stellar photosphere, whereas the subsequent re-emission is more or less isotropic, or, to be more precise, at least fore-aft symmetric with respect to the radial direction. This change in direction angle $\theta$ leads to a radial transfer of momentum, $\Delta P=h / c\left(v_{\text {in }} \cos \theta_{\text {in }}-v_{\text {out }} \cos \theta_{\text {out }}\right)$ (for details, see, e.g., Puls 1987), and this angle change (together with the Doppler-effect, see below) is the key of the momentum transfer and associated radiative line acceleration.

When integrating this momentum transfer over all scatterings, the losses or gains due to re-emission processes cancel because of fore-aft symmetry, resulting in a radially directed, total line acceleration, $g_{\text {rad }}^{\text {line }}$. This acceleration acts on the total wind plasma, as long as the momentum gained by the metal ions can be shared with the more abundant ions of $\mathrm{H}$ and/or He which are (usually) less accelerated due to far fewer lines close to the stellar flux maximum.

Coulomb coupling. For denser winds, the momentum transfer from the active metal ions to the passive hydrogen and helium ions is easily fulfilled via Coulomb collisions, but this may no longer hold for tenuous winds. The one-fluid approximation is applicable as long as the active ions are slowed down efficiently through electric charge interactions with the passive particles, i.e., if the relevant time scale for momentum transfer due to collisions is small in comparison to the time the active ions (accelerated by line acceleration) need to drift apart from the passive ones. For a one-component medium, this drift velocity, $v_{\mathrm{d}}$, scales with $\rho^{-1}$ (with density $\rho$; for details, see Springmann 1991). Thus, decoupling $\left(v_{\mathrm{d}}>v_{\text {th }}\right.$, with $v_{\text {th }}$ the thermal velocity, here for the dominant passive ion) and subsequent ionic runaway can be expected to become most relevant for stars with small mass-loss rates and large terminal wind velocities $v_{\infty}$ (Springmann and Pauldrach 1992, Babel 1995, 1996, Krtička and Kubát 2000, Owocki and Puls 2002, Krtička et al 2003).

Basic assumptions, equation of motion. In order to predict the physical structure of a line-driven wind, let us first employ the so-called standard model. This model assumes the wind to be stationary, homogeneous (e.g., no shocks or clumps) and spherically symmetric, which is justified as long as rotation and magnetic fields (covered in Sect.2.2) can be neglected, since all external forces are purely radial.

In this case, the total mass flux (or mass-loss rate) through a spherical shell with radius $r$ surrounding the star is conserved, as may be seen from the equation of continuity

$$
\dot{M}=4 \pi r^{2} \rho(r) v(r)=\text { constant. }
$$

The corresponding (stationary) momentum equation reads

$$
v \frac{\mathrm{d} v}{\mathrm{~d} r}=-\frac{G M}{r^{2}}-\frac{1}{\rho} \frac{\mathrm{d} p}{\mathrm{~d} r}+g_{\mathrm{rad}},
$$


with inwards directed gravitational acceleration and outwards directed pressure ( $p$ ) term and radiative acceleration. The necessary condition for initiating a stellar wind is that the total radiative acceleration, $g_{\mathrm{rad}}=g_{\mathrm{rad}}^{\text {line }}+g_{\mathrm{rad}}^{\text {cont }}$, exceeds gravity beyond a certain point in the outer photospheric layers. With the equation of state, $p=a^{2} \rho$, where $a$ is the isothermal speed of sound, and using Eq. 11 the equation of motion is given by

$$
\left(1-\frac{a^{2}}{v^{2}}\right) v \frac{\mathrm{d} v}{\mathrm{~d} r}=\frac{2 a^{2}}{r}-\frac{\mathrm{d} a^{2}}{\mathrm{~d} r}-\frac{G M}{r^{2}}+g_{\mathrm{rad}}
$$

The radiative acceleration. The prime challenge lies in accurately predicting the $g_{\text {rad }}$ term in the equation of motion. For free electrons (the Thomson acceleration), this is simply proportional to the Thomson opacity, $\sigma_{\mathrm{e}}=s_{\mathrm{e}} \rho\left(s_{\mathrm{e}}\right.$ proportional to cross section) times the flux:

$$
g_{\text {rad }}^{\mathrm{Th}}=\frac{1}{c \rho} \frac{\sigma_{\mathrm{e}} L}{4 \pi r^{2}}=g_{\text {grav }} \Gamma,
$$

with Eddington's $\Gamma$. However in reality line scattering is the more dominant contributor to the overall radiative acceleration. The reason is that (i) line scattering is intrinsically much stronger than electron scattering, due to the resonant nature of bound-bound transitions (Gayley 1995), and (ii) that although photons and matter are only allowed to interact at specific frequencies, they can be made to resonate over a wide range of stellar wind radii via the Doppler effect (see reviews by Abbott 1984, Owocki 1994a).

For a single line at frequency $v$ and line optical depth $\tau$ illuminated by radially streaming photons, the line acceleration can be approximated in terms of purely local quantities within the so-called Sobolev theory (Sobolev 1960). Under typical conditions, this approximation applies for the supersonic part of the wind, and, to a lesser extent, also for the transonic region as long as opacity, source function and velocity gradient do not change significantly over a velocity interval $\Delta v=v_{\text {th }}$, corresponding to a geometrically region of width $\Delta r \approx v_{\text {th }} /(\mathrm{d} v / \mathrm{d} r)=: L^{\mathrm{Sob}}$, the so-called Sobolev length. Insofar, the line optical depth in expanding atmospheres has an entirely different character than in hydrostatic photospheres: in winds, the interaction between photon and absorbing atom is restricted to a region of width $L^{\text {Sob }}$, which is usually very narrow, and the line optical depth becomes an (almost) local quantity.

Using this approximation, the line acceleration can be expressed as

$$
g_{\text {rad }, \mathrm{i}}^{\text {line }}=\frac{L_{v} v}{4 \pi r^{2} c^{2}}\left(\frac{\mathrm{d} v}{\mathrm{~d} r}\right) \frac{1}{\rho}\left(1-e^{-\tau}\right),
$$

where $L_{V}$ is the luminosity at line frequency, and the (radial) line optical depth, in the Sobolev approximation, is

$$
\tau \approx \tau^{\mathrm{Sob}}=\bar{\chi} \lambda /(\mathrm{d} v / \mathrm{d} r),
$$

with $\bar{\chi}$ the frequency integrated line-opacity and $\lambda$ the transition wavelength. Note that for optically thin lines, $\tau<1$, the acceleration becomes indendent of the velocity field and recovers the same functional form as Eq. 4, with $\sigma_{\mathrm{e}} L$ replaced by 
$\bar{\chi} L_{v}$, whereas for optically thick lines, $\tau>1$, the line acceleration depends on the spatial velocity gradient $\mathrm{d} v / \mathrm{d} r$. This dependence (unique in physics and being a consequence of the Doppler effect) is the origin of a number of peculiar features related to radiative line driving, as we will see in Sect.6

The final exercise is now to sum over all contributing lines, which, following the ideas by Castor et al (1975, "CAK"), is conventionally done by using so-called line-strength distribution functions, which describe the statistical dependence of the number of lines on frequency position and line-strength, $k_{\mathrm{L}}$,

$$
k_{\mathrm{L}}=\frac{\bar{\chi} \lambda}{\rho} \frac{1}{s_{\mathrm{e}} v_{\mathrm{th}}} .
$$

$k_{\mathrm{L}}$ measures the line opacity in units of the Thomson opacity and, for resonance lines and frozen in ionization, remains constant throughout the wind. From investigating an ensemble of C III lines (in LTE), CAK modeled the corresponding distribution function by a power law,

$$
d N\left(v, k_{\mathrm{L}}\right)=-N_{o} f_{v}(v) k_{\mathrm{L}}{ }^{\alpha-2} \mathrm{~d} v \mathrm{~d} k_{\mathrm{L}}
$$

with $0<\alpha<1, N_{o}$ a normalization constant and $f_{v}(v)$ the frequency distribution, which is rather independent from the distribution in line-strength. More elaborate calculations including ions from all participating elements have confirmed this approach (see Fig. 1, left panel).

Combining the radiative line acceleration (Eq. 5) and the distribution of lines, (Eq. 8), the total line acceleration can be calculated by integration, and, following CAK, expressed in terms of the Thomson acceleration (Eq. 4) multiplied by a factor $M$, the so-called force-multiplier,

$$
\frac{g_{\mathrm{rad}}^{\text {line }}}{g_{\mathrm{rad}}^{\mathrm{TH}}}=M(t)=k t^{-\alpha} \propto\left(\frac{\mathrm{d} v / \mathrm{d} r}{\rho}\right)^{\alpha}
$$

(for details see, e.g., Puls et al 2000, and for an alternative, sometimes advantageous, formulation Gayley 1995). The optical depth parameter, $t$,

$$
\tau=t k_{\mathrm{L}} ; \quad t=\frac{s_{\mathrm{e}} v_{\mathrm{th}} \rho}{\mathrm{d} v / \mathrm{d} r}=\frac{1}{k_{\mathrm{L}}(\tau=1)}
$$

is the optical depth of a line with an opacity equal to Thomson-scattering, or, alternatively, corresponds to the inverse of the line-strength of a line with unit optical depth. Because of the power-law distribution, the line-acceleration is dominated by lines distributed around a line-strength $k_{\mathrm{L}}(\tau=1)=t^{-1}(r)$ ( \pm 1 to 2 dex above and below this value, depending on $\alpha$ ).

The force-multiplier parameter $k$ is proportional to the effective, i.e., fluxweighted number of lines stronger than Thomson-scattering, $N_{\text {eff }}{ }^{2}$ and can be interpreted as the fraction of flux that would be blocked already in the photosphere if all lines were optically thick. $\alpha$ does not only correspond to the slope of the line-strength distribution, but, more generally, quantifies also the ratio of the

2 For the above power-law distribution, $N_{\text {eff }}=N_{o} /(1-\alpha) \times \int_{0}^{\infty}\left(L_{v} v / L\right) f_{v}(v) \mathrm{d} v, k=$ $\left(v_{\text {th }} / c\right) \Gamma(\alpha) \times N_{\text {eff }}$, with $v_{\text {th }}$ the thermal speed of a representative ion (chosen as hydrogen by CAK) and Gamma-function $\Gamma(\alpha) \approx 1 / \alpha$ for the values considered here. 
line acceleration from optically thick lines to the total one. In case of deviations from a power-law distribution, the radiative acceleration at location $r$ is controlled by its local slope $\alpha$ around $k_{\mathrm{L}}(\tau=1)$ (Puls et al 2000).

This line force description can easily be extended with an additional dependence via $\left(n_{\mathrm{e} 11} / W\right)^{\delta}$ to account for ionization effects (Abbott 1982), in the realistic case that the ionization is not frozen in $\left(n_{\mathrm{e} 11}\right.$ is the electron density in units of $10^{11} \mathrm{~cm}^{-3}$, and $W$ the dilution factor of the radiation field). Under typical Ostar conditions, $\delta$ is small (of the order of 0.1, see below), but can reach larger (positive or even negative) values if either the ionization equilibrium changes significantly throughout the wind (which might happen in dense winds) or the wind is mostly driven by hydrogen and helium lines, in case of extremely metal-poor stars (see page 11). Note that in any case $\delta$ is not a free parameter, but has to be determined - in parallel with the other two force-multiplier parameters $k$ and $\alpha$ - in a self-consistent way from the (NLTE-) line opacities and the radiation field (see, e.g., Pauldrach et al 1994).

Finally, the so-called finite cone angle correction factor has to be included to account for non-radially streaming photons (Pauldrach et al 1986, Friend and Abbott 1986).

Solving the equation of motion. Also for the complete line sample, the radiative acceleration depends on $\mathrm{d} v / \mathrm{d} r$ (now through a power of $\alpha$, Eq. 9), and it is conventionally assumed that this term has a similar meaning as the velocity gradient entering the inertial term on the left hand side (hereafter lhs) of Eq. 3 (but see Lucy 1998). In this case, the equation of motion becomes non-linear in the variable $y=r^{2} v \mathrm{~d} v / \mathrm{d} r$, and can be approximated by

$$
y=-G M(1-\Gamma)+f k L \dot{M}^{-\alpha} y^{\alpha}
$$

where $f$ is either constant (in the CAK case) or depends on $(r, v, \mathrm{~d} v / \mathrm{d} r, \dot{M})$ if one includes all subtleties. As the enthalpy term in the energy equation is much smaller than the potential and kinetic energy, the above equation has been simplified by neglecting the gas pressure. For constant $f$, this equation can be easily solved, and also the complete equation provides no real difficulty (see CAK and Pauldrach et al 1986). Note that these solutions involve the presence of a critical point which controls the mass-loss rate. For models including non-radial photons, this critical point is located close to the stellar photosphere and connects a somewhat shallow (with respect to $y$ ), sub-critical flow smoothly with a steeper, supercritical one. For further discussion regarding solution topologies and non-Sobolev line-forces, we refer the reader to Poe et al (1990), Owocki and Puls (1999), Feldmeier and Shlosman (2000, 2002) and Madura et al (2007).

As a final result, one obtains the following velocity law and scaling relations for $v_{\infty}$ and $\dot{M}$ :

$$
\begin{aligned}
& v(r)=v_{\infty}(1-R / r)^{\beta}, \quad\left\{\begin{array}{l}
\beta=0.5 \text { (CAK case) } \\
\beta=0.8 \text { (O-stars) ..2 (BA-supergiants) }
\end{array}\right. \\
& v_{\infty}=C_{\infty}\left(\frac{2 G M(1-\Gamma)}{R_{*}}\right)^{\frac{1}{2}}=C_{\infty} v_{\mathrm{esc}}, \quad \begin{cases}C_{\infty}=\left(\frac{\alpha}{1-\alpha}\right)^{0.5} & \text { CAK case } \\
C_{\infty} \approx 2.25 \frac{\alpha}{1-\alpha} & \text { incl. all details }\end{cases}
\end{aligned}
$$




$$
\dot{M} \propto(k L)^{1 / \alpha^{\prime}}(M(1-\Gamma))^{1-1 / \alpha^{\prime}}
$$

with photospheric escape velocity $v_{\text {esc }}$ (already corrected for the Thomson acceleration!) and $\alpha^{\prime}=\alpha-\delta$. For O-stars, $\delta$ is usually small (order of 0.1 ), $\alpha^{\prime} \approx 0.6$ and $k$ of the order of 0.1. From Eq. 12, we can also derive the behaviour of the optical depth parameter, $t$. It is proportional to $\left(v(r) / v_{\infty}\right)^{1 / \beta-2}$, i.e., remains constant throughout the wind for the CAK case, and increases from outside to inside for $\beta>0.5$.

These relations (e.g., Kudritzki et al 1989; for first order corrections to Eq.13 see Kudritzki and Puls 2000, their Eq. 15) compare very well with numerical results from integrating the complete equation, but are only valid for spatially constant force multiplier parameters, $k, \alpha$ and $\delta$. Kudritzki (2002) provides elaborate additions to consider depth dependent parameters as well.

Further assumptions involve the adoption of a core-halo structure that neglects continuum formation in the wind (important for dense winds), and the neglect of multi-line effects: in the approach as described above, each line is allowed to interact with unattenuated photospheric continuum radiation, irrespective of its frequency separation from other lines. This neglect leads to overestimated radiation forces in most cases (Friend and Castor 1983, Abbott and Lucy 1985, Puls 1987, Lucy and Abbott 1993, Springmann 1994, Gayley et al 1995, Vink et al 2000). In Sect. 3. most of these assumptions will be relaxed and more quantitative predictions will be provided. Note, however, that the basic scaling relations remain rather unaffected by these improvements.

The wind-momentum luminosity relation. Using the above scaling relations, one can construct a modified wind momentum rate, $D_{\text {mom }}=\dot{M} v_{\infty}\left(R_{*} / R_{\odot}\right)^{1 / 2}$. Given that $v_{\infty}$ scales with the escape velocity (Eq. 13), $D_{\text {mom }}$ scales with luminosity and effective line number alone,

$$
D_{\mathrm{mom}}=\dot{M} v_{\infty}\left(\frac{R_{*}}{R_{\odot}}\right)^{\frac{1}{2}} \propto\left(k \frac{L}{L_{\odot}}\right)^{\frac{1}{\alpha^{\prime}}}\left(\frac{M}{M_{\odot}}(1-\Gamma)\right)^{\frac{3}{2}-\frac{1}{\alpha^{\prime}}} \propto\left(k \frac{L}{L_{\odot}}\right)^{\frac{1}{\alpha^{\prime}}},
$$

as long as $\alpha^{\prime} \simeq 2 / 3$ (remember that $\alpha^{\prime} \approx 0.6$ for O-star). In this case, the effective stellar mass $M(1-\Gamma)$ cancels in the product $\dot{M} v_{\infty}$ when applying the scaling relations, which is rather convenient since masses are difficult to determine from observations and, moreover, seem to disagree when compared with evolutionary predictions (e.g., Puls 2008 and references therein). Taking the logarithm of Eq. (15) and assuming $\alpha^{\prime} \simeq 2 / 3$, we find

$$
\log D_{\text {mom }} \approx x \log \left(L / L_{\odot}\right)+D
$$

(with slope $x$ and offset $D$, depending on the flux-weighted number of driving lines), which is known as the "Wind momentum luminosity relationship (WLR)" (Kudritzki et al 1995, Puls et al 1996) and can be considered to be one of the big triumphs of the radiation-driven wind theory. This relationship can, e.g., be utilized to determine extragalactic distances by purely spectroscopic means (Kudritzki et al 1999, Kudritzki and Puls 2000, but see Sect.4.2.1), and it also played an instrumental role in determining the empirical mass-loss metallicity dependence for $\mathrm{O}$ stars in the Local Group, which will be discussed in Sect. 4. Equally important, 
observed and theoretically predicted WLRs can be compared to test the predictions and validity of the radiation driven wind theory for various spectral types, and to identify present shortcomings (e.g., Sect. 5).

Predictions from line-statistics. What determines the slope? One may now ask what controls the slope of the line-strength distribution function (Eq. 8), and in particular, why is $\alpha \approx 2 / 3$ ? Since the line-strength $k_{\mathrm{L}}$ is proportional to the product of absorber density per unit mass (depending on abundance, ionization fraction and excitation) and oscillator-strength, the answer to this question is rather difficult, in particular since a large fraction of the line acceleration, at least in the lower wind, is due to lines from iron group elements (see below) with a complex electronic configuration. In the following, we will highlight certain aspects (for details, see Puls et al 2000).

Most interestingly, it is rather easy to show that $\alpha$ would be almost exactly $2 / 3$ if all lines would originate from hydrogenic ions and would be resonance lines, i.e., excitation effects could be neglected. Then the line-strength distribution function depends solely on the distribution of the oscillator strengths $f$, and from the well known Kramers-formula for hydrogenic ions with transitions $1 \rightarrow n$ (with principal quantum number $n$ ) and corresponding selection rules, one finds that $f(1, n) \approx$ const $/ n^{3}$ and $\mathrm{d} N / \mathrm{d} f \propto f^{-4 / 3}$. Since, under the discussed conditions, this slope corresponds to $\alpha-2$, we immediately obtain $\alpha=2 / 3$.

Similarly important is the result that for trace ions one stage below the major ionization stage the equality $\alpha+\delta \approx 1$ is valid throughout the wind. Thus, for hot, metal-poor stars driven predominantly by $\mathrm{H}$ I and He II, the $\delta$-term can become much more influential on the wind solution than in "conventional" winds driven by ions of the dominant stage, where $\delta \approx 0.1$. This analytical result has been proven by Kudritzki (2002) by means of consistent wind calculations, and is of relevance in the context of radiation driven winds from the First Stars. Note that in these extreme cases the scaling relations for $\dot{M}$ and $v_{\infty}$ as provided above become invalid, since they are based on small values for $\delta$.

For non-hydrogenic ions (with many and low lying levels), the situation is more complicated. Per ion, the typical line-strength distribution consists of two parts, a steeper one dominated by excitation effects and a flatter one following the oscillator strength distribution, which, after summing up over all participating ions, becomes almost irrelevant. Most important for the shape of the total distribution is the difference in line-statistics between iron group and light ions as well as their different (mean) abundances. Since the former group comprises a large number of meta-stable levels, the line number from iron group elements is much higher, especially at intermediate and weak line-strengths. Additionally, this number increases significantly with decreasing temperature (more lines from lower ionization stages), which becomes important with respect to the so-called bi-stability mechanism (see below).

For solar abundances, iron group elements dominate the distribution at low and intermediate values of line-strengths, i.e., they dominate the acceleration of the inner wind (large $t$ ) and thus determine the mass-loss rate. Lines from light ions (including hydrogen under A-star conditions), on the other hand, populate the high $k_{\mathrm{L}}$ end and accelerate the outer wind (low $t$ ), i.e., determine $v_{\infty}$. Typically, this part of the distribution is steeper than the rest (e.g., Fig. 1), due to excitation effects, 

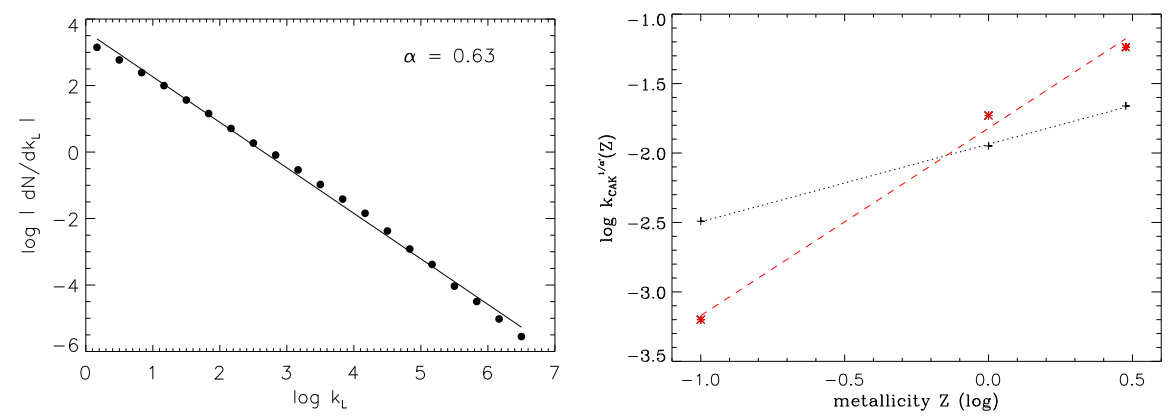

Fig. 1 Left: Logarithmic plot of the line-strength distribution function for an O-type wind of $40 \mathrm{kK}$ (solar abundance), and corresponding power-law fit.

Right: Predictions from line-statistics. Dependence of mass loss (via $k^{1 / \alpha^{\prime}}$, cf. Eq. 17) on metallicity, for $T_{\text {eff }}=40 \mathrm{kK}$ (dotted) and $T_{\text {eff }}=10 \mathrm{kK}$ (dashed). The slopes are 0.56 and 1.35, respectively. Data from Puls et al (2000, Table 3).

and only a small number of lines (Krtička 2006: "a few dozens") is responsible for this acceleration! Insofar, the rather large observed variance in $v_{\infty} / v_{\text {esc }}$ even for stars of similar spectral type (Sect. 4.2.1) is not surprising, since subtle effects (small variations in temperature, density, composition - cf. Pauldrach et al 1990, their Fig. 8 - and also X-rays, see Sect. 6.1) can have a significant effect on the population and line-strengths of light ions.

Note finally that the above results, which are based on the investigation of linestrength distribution functions alone, have been confirmed by direct wind calculations, see Pauldrach (1987), Vink et al (1999, 2001) and Krtička (2006).

Predictions from line-statistics. Dependence on temperature, wind-density and metallicity. Summarizing the above paragraphs, deviations from a perfect power law are to be expected, mainly due to the different distributions of "light" and "heavy" ions, their specific dependence on temperature and the fact that the distribution at the high $k_{\mathrm{L}}$ end is controlled by excitation effects for the predominant ion species, inducing a steeper slope. Translated to the effective value of $\alpha$ (i.e., evaluated at $k_{\mathrm{L}}=t^{-1}$ ) which controls the line-force (and the slope of the WLR), this quantity should decrease with decreasing $T_{\text {eff }}$, decreasing wind density and decreasing metallicity $Z$. In all these cases, the WLR is expected to become steeper than in the "standard" situation encountered for O-stars where $\alpha$ is of the order of 0.6...0.7. For A-supergiants, e.g., line-statistics predict $\alpha \approx 0.45$ such that in addition to a steeper WLR also the scatter introduced by a non-vanishing mass term should become significant.

Besides the variation of $\alpha$ with $Z$, the major consequence of a different metallicity is a variation of $k$, i.e., a different effective line-number. Roughly speaking, the higher the metallicity, the more effective lines are to be expected, and vice versa. Since $k_{\mathrm{L}}$ varies proportionally to $Z$, as long as the ionization equilibrium remains rather unaffected from the different metallicity, the assumption of a powerlaw line-strength distribution function (Eq. 8) immediately implies $N_{o}(Z) \propto Z^{1-\alpha}$, with similar scalings for $k$ and $N_{\text {eff. }}$ By means of Eq.14, the mass-loss rate should 
then scale according to

$$
\dot{M}(Z) \propto Z^{\frac{1-\alpha}{\alpha^{\prime}}} .
$$

From Fig. 11 right panel, we see that the predicted dependence of $k(Z) \propto Z^{1-\alpha}$ is actually present, and the derived slopes $(s=(1-\alpha) /(\alpha-\delta))$ are consistent with prototypical values, namely $\left(\alpha, \alpha^{\prime}\right)=(0.68,0.58)$ for the O-type and $\left(\alpha, \alpha^{\prime}\right)=(0.48$, 0.38 ) for the A-type models, respectively (lower $\alpha$ because of lower $T_{\text {eff }}$ : more Fe lines from lower stages). Moreover, these numbers are also consistent with results derived from the analysis of a large sample of O-stars in the Galaxy and the Magellanic Clouds (Sect.4.2.1). Detailed measurements of the metallicity dependence of A-supergiant winds are still missing though (but see page 50).

The bi-stability mechanism. Most of the previous paragraphs may have given the reader the impression that - save for their intrinsic luminosity dependence - the mass-loss properties from the winds of massive stars are more or less identical, but nothing is further from the truth: the wind properties of massive stars are intriguingly diverse! This diversity is, of course, related to the variation of effective line number and $\alpha^{\prime}$ as a function of spectral type and metallicity and to the different efficiencies of multi-line effects as a function of wind density.

Of particular interest is the so-called bi-stability mechanism, which was first encountered in the model calculations of the famous Luminous Blue Variable (LBV) P Cygni by Pauldrach and Puls (1990). At a critical effective temperature around $21000 \mathrm{~K}$ (Lamers et al 1995), the overall properties of $v_{\infty}$ and $\dot{M}$ are expected to change drastically. Vink et al (1999) showed that when a massive star evolves from hot temperatures to lower ones, the dominant wind driving element of iron $(\mathrm{Fe})$ recombines from FeIV to Fe III, which is predicted to result in an increase of $\dot{M}$ by a factor 5 and a drop of $v_{\infty}$ by a factor 2 .

As was pointed out already by Pauldrach and Puls (1990), such a recombination can be triggered by the optical depth of the hydrogen Lyman continuum 3 As long as this is optically thin (e.g., for hotter temperatures), the EUV continuum is rather strong, such that most iron resides in Fe IV, which has fewer lines than Fe III, resulting in a lower density, faster wind. When the Lyman continuum becomes optically thick, there is almost no EUV flux left, and iron recombines to Fe III with more lines, inducing a dense, low velocity wind. Since the optical depth of the Lyman continuum is closely coupled to wind density via recombination effects,

$$
\tau_{\mathrm{Ly}} \propto \rho^{2} \propto\left(\frac{\dot{M}}{v_{\infty}}\right)^{2} \propto(M(1-\Gamma))^{1-2 / \alpha^{\prime}} \approx(M(1-\Gamma))^{-2 \ldots-3},
$$

the abrupt (see below) change in wind conditions does not only depend on $T_{\text {eff, }}$, but also, and severely, on effective mass (e.g., Figs. 4 and 5 in Pauldrach and Puls 1990). As we will see in Sect.2.2.1 this can play an important role in understanding the $\mathrm{B}[\mathrm{e}]-$ star phenomenon.

\footnotetext{
3 At least for dense winds such as from P Cygni considered in the original explanation. For most "normal" massive stars as studied by Vink et al (1999, 2000), the Lyman continuum remains optically thin between photosphere and critical point, and the abrupt change of the Fe ionization equilibrium is rather due to conventional temperature effects and the runaway in density as described below. For these objects we consider $\tau_{\mathrm{Ly}}$ as an alias for the Fe III/Fe IV ionization equilibrium.
} 
The reason that the wind should react to the different accelerating conditions by an actual "jump" in $\dot{M}$ and $v_{\infty}$ is that the physics of the line driving implies that when the density increases due to an increasing $\dot{M}$, the inner wind is anticipated to recombine even more, leading to a run-away mass-loss increase until all iron has accumulated in Fe III. The line-driving in the outer wind is expected to react to an increased inner density by lowering its terminal wind velocity (remember that $g_{\text {rad }}^{\text {line }} \propto \rho^{-\alpha}$, Eq. 9]. In Sect. 4.2.1 we will discuss the empirical constraints on the bi-stability mechanism.

Also here, the spectral-type dependence of the mass-loss rate and terminal velocity occurs in conjunction with changes in $N_{\text {eff }}$ and $\alpha^{\prime}$, which could subsequently be reflected in the slope and offset of the WLR. Let us emphasize that the spectral-type dependence of the WLR represents a most active aspect of hot-star wind research (Benaglia et al 2007, Markova and Puls 2008).

\subsection{The impact of rotation and magnetic fields}

All previous results have been derived under the assumption of spherical symmetry, with gravity and radiative acceleration as the only external forces. In the following sections, we will summarize alterations due to rotation and magnetic fields, if present.

\subsubsection{Rotation and stellar winds}

(Almost) all massive stars start their evolution as rapid rotators, and remain rapidly rotating during the largest part of their life-time, at least with respect to the most decisive quantity, namely the so-called "critical ratio" between surface rotation $v_{\text {rot }}$ and critical velocity (Meynet and Maeder 2000). $v_{\text {rot }}$ itself decreases (almost) continuously during the main sequence and evolved phases, where the rate of this decrease is larger for the more massive stars due to the larger mass loss and accompanying larger removal of angular momentum. For supergiants, the reduction is due to an increasing stellar radius as well.

The role of rotation as a fundamental stellar parameter of massive stars has been highlighted by Langer et al (1997), and effects on stellar structure and evolution were detailed by the Geneva group (A. Maeder, G. Meynet and co-workers) in a series of publications. For corresponding reviews, we refer to Maeder and Meynet (2000b, 2003b) (see also material presented in Wolf et al 1999 and Maeder and Eenens 2004).

Rotation affects the physics and diagnostics of line-driven winds from massive stars at least in three ways: (i) the dynamics is modified, (ii) the diagnostics, particularly for $\dot{M}$, needs to consider rotation due to deviations from spherical symmetry, and (iii) line-profile variability is induced due to an interplay between disturbances in the photosphere (stellar spots, non-radial pulsations), rotation and wind-dynamics (co-rotating interaction regions, CIRs, see Sect.6.4).

1-D models, scaling relations. First attempts to include rotation into the stellar wind dynamics were based on a 1-D solution in the equatorial plane, and have 
been performed by Friend and Abbott (1986) and Pauldrach et al (1986), with applications regarding outflowing disks of Be-stars by de Araujo (1995), and investigations of the solution topology by Curé and Rial (2004, see also Curé 2004 and Madura et al 2007).

Under the assumption of purely radial line-forces, the equation of motion is governed by central forces, i.e., the angular momentum is conserved, $L \propto r v_{\phi}=$ const, and the azimuthal velocity in the equatorial plane is given by $v_{\phi}(r)=$ $v_{\text {rot }}\left(R_{*}\right) R_{*} / r$, with polar velocity $v_{\theta}(r, \theta=90)=0$ and co-latitude $\theta$. Thus, the equation of motion remains almost identical to Eq. 3 or the simplified version Eq. 11, except that the effective gravity (here defined as the difference between gravitational and Thomson acceleration) has to be corrected for the centrifugal acceleration $v_{\phi}^{2} / r$,

$$
-\frac{G M(1-\Gamma)}{r^{2}} \rightarrow-\frac{G M(1-\Gamma)}{r^{2}}\left(1-\Omega^{2} \frac{R_{*}}{r}\right) \quad \Omega=\frac{v_{\mathrm{rot}}\left(R_{*}\right)}{v_{\text {crit }}}
$$

with $v_{\text {crit }}$ the 1-D critical velocity $4, v_{\text {crit }}=v_{\text {esc }} / \sqrt{2}$. In the following, we will mostly consider models not too close to critical rotation $(\Omega \lesssim 0.75)$. For a discussion of more extreme situations in one dimension, see Curé (2004), Curé and Rial (2004) and Madura et al (2007). Since in the former case the critical point (page 9) of the non-rotating solution remains almost unaffected by the centrifugal term due to $r_{\text {crit }} \approx R_{*}$ (e.g., Pauldrach et al 1986, Owocki and ud-Doula 2004, Madura et al 2007), the effective mass can be replaced by $M(1-\Gamma)\left(1-\Omega^{2}\right)$, and $\dot{M}$ scales, to a very good approximation, with

$$
\dot{M}(\Omega) \approx \dot{M}(0)\left(1-\Omega^{2}\right)^{1-1 / \alpha^{\prime}} .
$$

Similarly, since $v_{\infty}$ scales with $v_{\mathrm{esc}}$, a rather good approximation for the dependence of $v_{\infty}$ on $\Omega$ is

$$
v_{\infty}(\Omega) \approx v_{\infty}(0)\left(1-\Omega^{2}\right)^{1 / 2} .
$$

For independent arguments leading to this equation, see Gayley (2000). Comparisons of exact vs. approximate solutions for $\dot{M}$ and $v_{\infty}$ as a function of $\Omega$ have been given, e.g., by Puls et al (1999).

Wind compressed disks and zones. In a seminal paper, Bjorkman and Cassinelli (1993) included the latitude dependence of the centrifugal forces into the previous 1-D description in order to generalize the approach. Since (at that time) it was still undebated to assume line-forces which are purely radially directed, under this assumption the angular momentum is conserved for each particle starting at a given co-latitude $0<\theta_{0}<\pi$, and its trajectory is restricted to an orbital plane tilted by $\theta_{0}$ with respect to the equatorial one. Thus, a 1-D solution is still possible per orbital plane, if one accounts for the centrifugal acceleration as a function of co-latitude,

$$
g_{\text {cent }}=\left(v_{\text {rot }}\left(R_{*}\right) \sin \theta_{0}\right)^{2} \frac{R_{*}^{2}}{r^{3}}
$$

\footnotetext{
4 for the radiatively reduced gravity as defined above and neglecting gravity darkening.
} 
and the scaling relations for the equatorial flow (Eqs. 20, 21) can be generalized with respect to $\theta_{0}$,

$$
\dot{M}\left(\Omega, \theta_{0}\right) \propto\left(1-\Omega^{2} \sin ^{2} \theta_{0}\right)^{1-1 / \alpha^{\prime}}, \quad v_{\infty}\left(\Omega, \theta_{0}\right) \propto\left(1-\Omega^{2} \sin ^{2} \theta_{0}\right)^{1 / 2} .
$$

In this description, $\dot{M}$ increases and $v_{\infty}$ decreases towards the equator, respectively. Since the particle is restricted to its orbital plane, the corresponding azimuthal angle, $\Phi^{\prime}(r)$, increases for increasing $r$ and can be calculated from integrating $\mathrm{d} \Phi^{\prime}(r) / \mathrm{d} r=v_{\phi}\left(r, \theta_{0}\right) /\left(r v_{r}\left(r, \theta_{0}\right)\right)$, using a $\beta$-velocity law (Eq. 12) in combination with $v_{\infty}\left(\theta_{0}\right)$ for the radial velocity component $v_{r}$.

$\Phi^{\prime}(r)$ may become large if either $v_{\text {rot }}$ is large, $v_{\infty}\left(\theta_{0}\right)$ is small, the velocity field is flat ( $\beta$ large) or the particles start from equatorial regions. In any case, however, the particles move towards the equator and, if $\Phi^{\prime}(r)$ became $\pi / 2$, would collide in the equatorial plane with particles from the opposite hemisphere which have started at similar (absolute) latitudes. Since the equatorwards directed motion corresponds to a non-vanishing polar velocity component in the stellar frame, and this component is small, but supersonic for large $\Phi^{\prime}$, a shock develops, and a "wind-compressed disk (WCD)" will be created, confined by the ram pressure of the wind. The WCD model has been confirmed in all aspects by numerical models using identical assumptions (Owocki et al 1994, and Fig. 2, left panel).

Even if the deflection in $\Phi^{\prime}$ is too small to allow for the creation of a disk, i.e., the equatorial plane cannot be reached by certain trajectories (because of too low rotation or too steep a velocity field) the above scaling relations (Eq. 23) remain valid, and a non-spherical, oblate wind is predicted, in this case denoted as a "wind-compressed zone (WCZ)" model. For such models, Petrenz and Puls (1996) have calculated $\mathrm{H}_{\alpha}$ profiles as a function of $v_{\text {rot }}$ and $v \sin i$, and concluded that standard 1-D diagnostics might lead to severe overestimates of $\dot{M}$ (50 to 70\%) in those cases were $v \sin i$ is small and the star is observed pole-on.

Non-radial line forces: the inhibition effect. After publication, the WCD model was regarded as a major step towards an explanation of disk phenomena in hot massive stars (e.g., Be-stars) until it became clear that it was based on an insufficient assumption (Owocki et al 1996, 1998a). Since, in a more-D situation, the optical depth depends on the directional derivative of the local velocity field (the radial gradient $\mathrm{d} v / \mathrm{d} r$ in Eq. 6 has to be replaced by $\mathbf{n} \cdot \nabla(\mathbf{n} \cdot \mathbf{v})$ ), and the radiative acceleration of strong lines is proportional to the integral over solid angle of this derivative times direction $\mathbf{n}$, the line force does not only depend on the radial velocity gradient, but also on other velocity gradients present in the flow. Most importantly, the dependence of $v_{r}$ on polar angle (increasing towards the pole, see Eq. (23) leads to a small but substantial polar acceleration, which is directed towards the pole as long as $\partial v_{r} / \partial \theta<0[$ i.e., as long as the equatorial outflow is slower than the polar one, which is (almost) inevitable for a rotating line-driven wind (due to the decrease of the effective, centrifugally corrected gravity towards the equator and $\left.v_{\infty} \propto v_{\mathrm{esc}}\right)$. This polar component of the line force,

$$
\frac{g_{\theta}^{\mathrm{rad}}}{g_{r}^{\mathrm{rad}}} \approx \frac{\alpha}{4} \frac{\partial v_{r} / \partial \theta}{\partial v_{r} / \partial r}\left(\frac{R_{*}}{r}\right)^{2}
$$

5 more precisely, if $\left(r \frac{\partial}{\partial r} \frac{v_{\theta}}{r}+\frac{1}{r} \frac{\partial v_{r}}{\partial \theta}\right)<0$ 

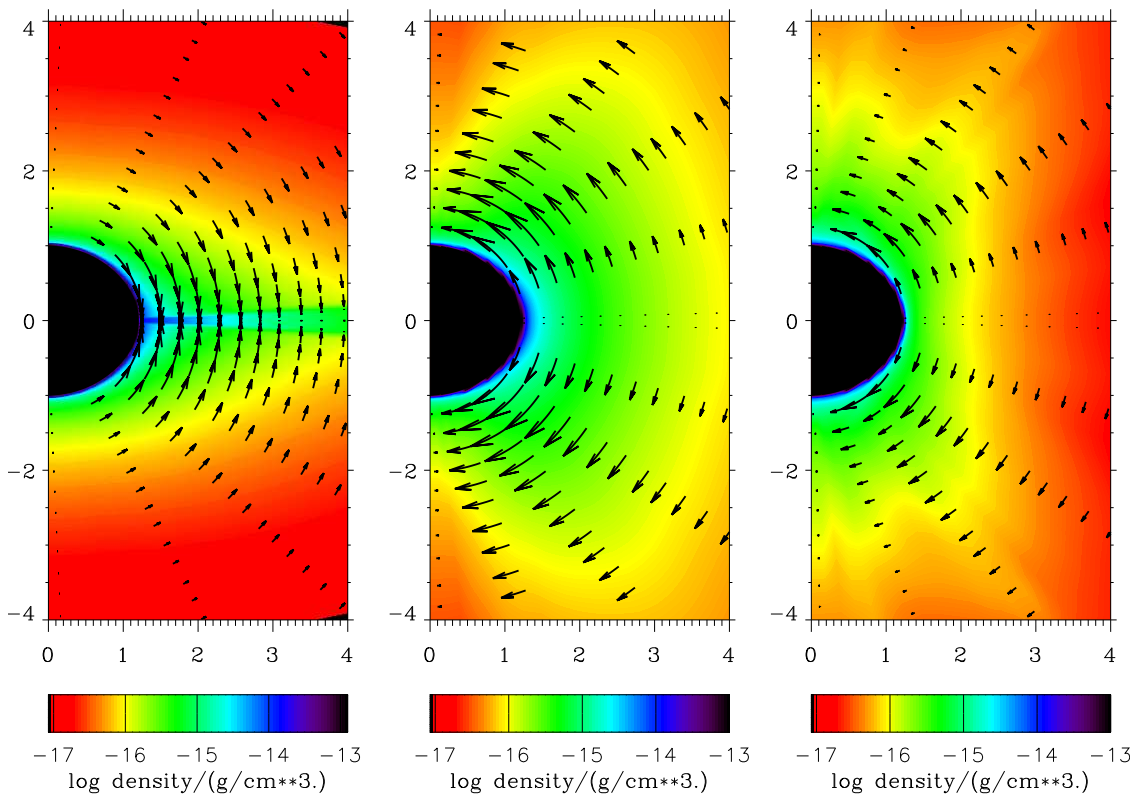

Fig. 2 Density and polar velocity of radiation driven wind models for a rapidly rotating B-dwarf, using different assumptions. The rotation axis is directed along the ordinate.

Left: radial line force $\rightarrow$ wind compressed disk. Middle: non-radial line force, uniform brightness temperature. Right: non-radial line force + gravity darkening.

Model parameters (polar values): $T_{\text {eff }}=20 \mathrm{kK}, \log g=4.11, R_{*}=4 R_{\odot}, \mathrm{M}=7.5 M_{\odot} \cdot v_{\text {rot }}=$ $350 \mathrm{~km} \mathrm{~s}^{-1}, v_{\text {esc }}($ pole $)=843 \mathrm{~km} \mathrm{~s}^{-1}, v_{\text {esc }}$ (equator) $=585 \mathrm{~km} \mathrm{~s}^{-1}, v_{\text {crit }}=487 \mathrm{~km} \mathrm{~s}^{-1}$ (see Eq. 26). From Petrenz (1999).

is sufficient to stop the small, equatorwards directed $v_{\theta}$ component of the WCD model and to induce a polewards directed velocity field, as shown by Owocki et al (1998a). This is the so-called "inhibition" effect (Owocki et al 1996), which can be seen in all numerical models including non-radial line forces (e.g., Owocki et al 1996 and Fig. 22 middle panel).

Gravity darkening: The $\Omega \Gamma$ limit and prolate winds. Because of the centrifugal forces, not only the wind is disturbed, but also the stellar surface distorted. The corresponding shape can be calculated from a Roche potential6 (see Collins 1963, Collins and Harrington 1966, and for applications Cranmer and Owocki 1995, Petrenz and Puls 1996). In this case, and with angular velocity $\omega$,

$$
\Omega=\frac{\omega}{\omega_{\text {crit }}}=\frac{v_{\text {rot }}}{v_{\text {crit }}} \frac{R_{\mathrm{eq}}^{\max }}{R_{\mathrm{eq}}(\Omega)}
$$

${ }^{6}$ First observations of such a surface distortion have been obtained by Domiciano de Souza et al (2003) for the rapidly rotating Be-star $\alpha$ Eri with the VLTI, resulting in a ratio between equatorial and polar radius of $1.56 \pm 0.05$. The shape of the observed distortion seems to indicate that the conventional assumption of a uniform rotation might not apply for this star. 
$\Omega$ becomes a function of equatorial radius $R_{\mathrm{eq}}$ (which itself is a function of $\Omega$ ). $\Omega=1$ (i.e., "total gravity" $=0$ at the equator, see below) occurs when the equatorial radius has reached its maximum extent, $R_{\mathrm{eq}}^{\max }=1.5 R_{\mathrm{p}}$, with polar radius $R_{\mathrm{p}}$.

The critical velocity, $v_{\text {crit }}$, requires special attention, due to the problem of gravity darkening. After some controversial discussions arising from the suggestion of an $\Omega$ limit by Langer (1997, 1998) which has been criticized by Glatzel (1998) (because of disregarding gravity darkening), Maeder and Meynet (2000a) performed a detailed study on the issue. According to this study, for not too large $\Gamma$ the critical speed has to be calculated from the actual mass (independent from $\Gamma$ ) and from the maximum equatorial radius, consistent with the arguments provided by Glatzel,

$$
v_{\text {crit }, 1}=\left(\frac{G M}{R_{\mathrm{eq}}^{\max }}\right)^{\frac{1}{2}}=\left(\frac{2 G M}{3 R_{\mathrm{p}}}\right)^{\frac{1}{2}} \quad \Gamma<0.639 .
$$

Note that this value is lower by a factor of $\sqrt{(2 / 3)}$ than in the corresponding 1-D case, due to the difference of polar and equatorial radius,

For such a distorted surface with latitude dependent effective gravity (in the following defined as gravity corrected for centrifugal terms), one has also to consider gravity darkening as a consequence of the von Zeipel-theorem (von Zeipel 1924) which states that the surface flux of a rotating star is proportional to the local effective gravity 7 Thus, the effective temperature depends on the latitude (in the example of Fig. [2, $T_{\text {eff }}$ (pole) $=21.3 \mathrm{kK}$ and $T_{\text {eff }}$ (equator) $=16.9 \mathrm{kK}$ ), which has to be accounted for in the calculation of the occupation numbers (through the radiation field).

The principle presence of gravity darkening has been recently confirmed by Monnier et al (2007), who combined the NIR radiation of the rapidly rotating A7V star Altair collected from four telescopes of the CHARA interferometric array to obtain an intensity image of the surface of this star. Interestingly, also their measurements (remember the problem for $\alpha$ Eri) showed a somewhat different temperature distribution compared to the standard approach assuming a uniformly rotating star and the von Zeipel theorem.

In any case, since the flux is modified by gravity darkening, also the $\Gamma$-term needs to be modified (Maeder 1999, Maeder and Meynet 2000a). Within the standard approach and to a good approximation, it turns out that

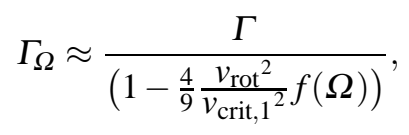

if one expresses the "total" gravitational acceleration (centrifugally corrected gravity, $g_{\text {eff }}$, minus radiative acceleration) as $g_{\text {tot }}=g_{\text {eff }}\left(1-\Gamma_{\Omega}\right)$, to preserve the formulations obtained so far. The function $f(\Omega)$ varies from 1 at low $v_{\text {rot }}$ to $0.813 \approx$

\footnotetext{
7 Though the original version of this theorem was restricted to the case of rotational laws that can be derived from a potential (e.g., cylindrical rotation), more recent investigations by Maeder (1999) have proven that this theorem is also applicable (within a 10\% error) to the case of "shellular" rotation $(\omega=\omega(r)$ for not too fast rotation) which has been proposed as the relevant rotational law at the surface levels of non-magnetic stars (Zahn 1992).
} 
$\sqrt{(2 / 3)}$ at critical rotation, and can be neglected in most cases. For large $\Gamma$ then, the critical speed needs to be redefined, and can be approximated (for exact expressions and details, see Maeder and Meynet 2000a) by

$$
v_{\text {crit }, 2} \approx v_{\text {crit }, 1}\left(\frac{3}{2}\right)^{1.25}(1-\Gamma)^{\frac{1}{2}} \quad \Gamma>0.639 .
$$

which results from the condition $g_{\text {tot }}=0$ when $\Gamma_{\Omega}=1$, the so-called $\Omega \Gamma$ limit (Maeder and Meynet 2000a): For large $\Gamma$, the critical velocity is significantly reduced by the proximity to the Eddington-limit. Thus, there is indeed a lower critical speed (as suggested by Langer 1997, 1998), at least when $\Gamma>0.64$, but it is important to emphasize that the $\Omega \Gamma$ limit occurs over all latitudes, not just near the equator, as assumed in the original idea of the $\Omega$ limit.

Note that the redefined $\Gamma_{\Omega}$-term needs to be considered in fast rotating models close to the Eddington-limit, a problem which has been neglected in all hydrodynamical wind calculations performed so far. In the present context, however, the most important effect of gravity darkening concerns the line force, due to the modified illumination of the lines. In a rotating wind illuminated by a uniformly bright disk, the mass flux increases from pole to equator according to Eq. $\left.{ }_{23}^{8}\right]^{8}$ The inclusion of gravity darkening into the luminosity dependence of the mass-loss rate (Eq. 14),

$$
L^{1 / \alpha^{\prime}} \rightarrow\left(F(\theta) R_{*}^{2}(\theta)\right)^{1 / \alpha^{\prime}} \underset{\propto}{\text { von Zeipel }}\left(g_{\text {eff }}(\theta) R_{*}^{2}(\theta)\right)^{1 / \alpha^{\prime}},
$$

thus leads to an additional dependence on $\left.\left(1-\Omega^{2} \sin ^{2} \theta\right)\right]^{1 / \alpha^{\prime}}$, such that in total

$$
\dot{M}(\theta) \propto L(\theta)^{1 / \alpha^{\prime}} M_{\text {eff }}(\theta)^{1-1 / \alpha^{\prime}} \propto\left(1-\Omega^{2} \sin ^{2} \theta\right)^{+1} \quad \text { (gravity darkening included) }
$$

the situation becomes reversed and the mass flux is predicted to increase from equator to pole, in parallel with the corresponding terminal velocity, inducing a prolate wind structure (cf. Owocki et al 1998a.b, Pelupessy et al 2000 and Fig. 2, right panel). The present-day stellar wind of $\eta$ Car has indeed been found to be elongated (Smith et al 2003a, van Boekel et al 2003) with a position angle equal to that of its ejecta.

Though the predicted angular dependence of the mass-loss rate is not affected by the modified $\Gamma_{\Omega}$-term (except for its influence on $\Omega$ via $v_{\text {crit }}$ ), this modification plays an important role when comparing the total, polar-angle integrated massloss rate with mass-loss rates from non-rotating models. To a similar degree of precision as valid for Eq. 27, one obtains (Maeder and Meynet 2000a)

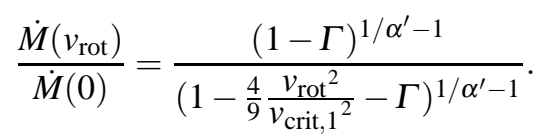

Thus, no dramatic influence of rotation on the total mass loss is to be expected in most cases. ?

\footnotetext{
${ }^{8}$ Note that the inhibition effect does not change this basic result, since it affects "only" the small polar velocity component and thus the disk formation.

9 The formal divergence at the $\Omega \Gamma$ limit is artificial, since in this case the material can no longer be lifted from the gravitational potential.
} 


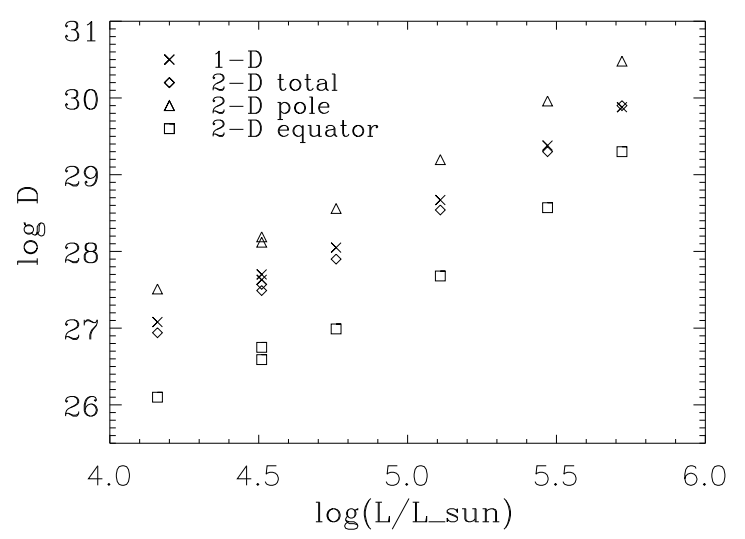

Fig. 3 Predicted WLR for rapidly rotating B-type stars $\left(v_{\text {rot }}=0.85 v_{\text {crit }}\right)$ with $T_{\text {eff }}=20 \mathrm{kK}$ and different radii. Compared are the modified wind-momenta $D_{\text {mom }}$ calculated from total (diamonds), equatorial (squares) and polar (triangles) mass-loss rates and average terminal velocities resulting from 2-D, NLTE simulations, and from corresponding 1-D models with $v_{\text {rot }}=0$ (crosses). From Petrenz and Puls (2000).

2-D NLTE models and the WLR of rapidly rotating stars. Strictly speaking, the scaling relations derived in the previous sections are only valid if the line-statistics remains unaffected from the modified wind structure. In order to investigate the influence of density, velocity and radiation field on the occupation numbers and lineforces as a function of $(r, \theta)$, Petrenz and Puls (2000) calculated self-consistent, 2-D, NLTE models for rapidly rotating B-star wind $\$$ T1, to investigate a spectral regime where the ionization structure is most sensitive to local conditions and variations of the radiation field. For all models, a prolate wind structure has been found, in accordance with the principal predictions from above.

As obvious from Fig. 3, the total wind momenta are hardly affected by rotation, since the increase in $\dot{M}, \mathrm{Eq}$. 30, is compensated by a decrease in the average $v_{\infty}$. Of course, the wind-momenta differ significantly when observed either pole or equator on. This problem can be mitigated when $\dot{M}$ diagnostics is used which scans mostly the lower wind region, e.g., $\mathrm{H}_{\alpha}$, since in these regions the density contrast between polar and equatorial regions is lower. Consequently, WLRs derived from 1-D analyses should remain rather unaffected from deviations from spherical symmetry, when samples of significant size are used, and objects with low $v \sin i$ (likely to be observed pole-on) are avoided. The scatter in the WLR increases due to rotational effects, of course.

B[e]-supergiants and the bi-stability. $\mathrm{B}[\mathrm{e}]$ supergiants (for an improved classification scheme, see Lamers et al 1998) show a hybrid spectrum (narrow Balmer lines and low ionization metal lines in emission, but also UV and Balmer P Cygni lines and a strong IR excess), which has been explained by an outflowing disk

\footnotetext{
10 computational feasible at that time because they applied a Sobolev line transfer and assumed an optically thin continuum.
} 

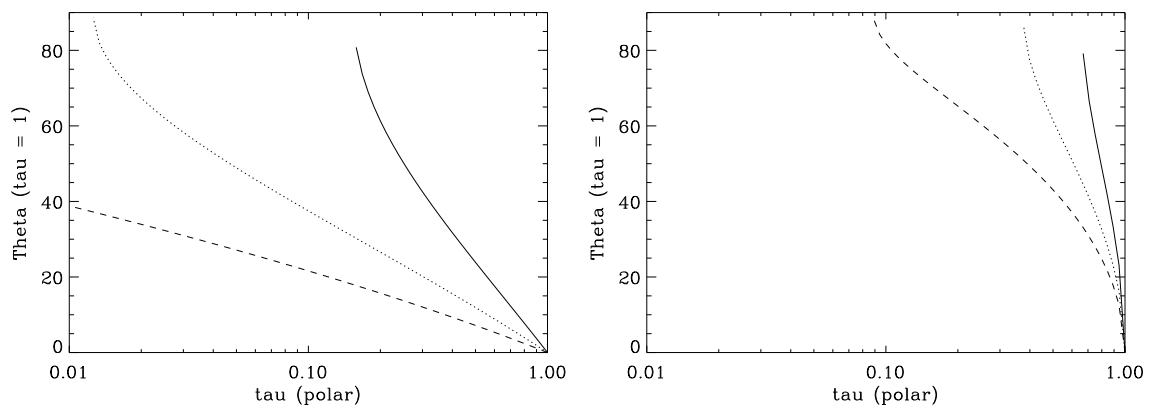

Fig. 4 Onset of bi-stability (co-latitude $\theta$ where $\tau_{\mathrm{Ly}}=1$ is reached) as a function of $\tau_{\mathrm{Ly}}$ (polar) and three rotational speeds, $\Omega=0.5,0.7,0.9$ (solid, dotted, dashed), for a B-supergiant with $T_{\text {eff }}=20 \mathrm{kK}$. Optical depth of Lyman continuum calculated according to Lamers and Pauldrach (1991). Left: original version. Right: mass-loss rate calculated including gravity darkening. The formation of a disk requires much larger polar mass loss and rotation than in the original version, see text.

with high density, low velocity and low ionization and a fast polar wind with low density and high ionization (Zickgraf et al 1986, 1989).

The origin of this phenomenon has been suggested by Lamers and Pauldrach (1991, see also Lamers et al 1999) to rely on a combination of rotation and bistability. Since, for a rotating wind, $\dot{M}$ was thought to increase and $v_{\infty}$ to decrease towards the equator (the impact of gravity darkening on $\dot{M}$ was yet unknown), the optical depth of the Lyman continuum

$$
\tau_{\mathrm{Ly}}=f\left[\left(\frac{\dot{M}}{v_{\infty}}\right)^{2}, T_{\mathrm{rad}}\right]
$$

becomes a strongly increasing function of $\theta$, particularly if including the von Zeipel theorem when calculating $T_{\mathrm{rad}}(\theta)$ which establishes the ionization equilibrium 11 Lamers and Pauldrach (1991) were able to parameterize the optical depth as a function of latitude-dependent gravity, $\tau_{\mathrm{Ly}} \propto\left(g_{\text {pole }} / g(\theta)\right)^{6.5}$. Because of the dependence of the bi-stability jump on this optical depth, the latitude where $\tau_{\mathrm{Ly}}$ reaches unity separates two regions, a polar one with low density and high velocity (predominantly driven by Fe IV) and an equatorial one with high density and low velocity, driven by Fe III (see Sect.2.1). The contrast between both regions results in an equatorial outflowing disk. From Fig. 4, left panel, one can see that even a thin polar wind with $\tau_{\mathrm{Ly}}=0.01$ (corresponding to $\dot{M}=0.35 \cdot 10^{-6} M_{\odot} \mathrm{yr}^{-1}$ ) can switch almost abruptly to $\tau_{\mathrm{Ly}}>1$ at $\theta \approx 40^{\circ}$ for $\Omega=0.9$ in the outlined scenario.

After the impact of gravity darkening on the mass-loss rate had been realized, Owocki et al (1998a) pointed out that the creation of $\mathrm{B}[\mathrm{e}]$ star disks is much more difficult than previously thought since $\dot{M}$ decreases equatorwards (Eq. 29], in contrast to the original approach. Thus, the increase of $\tau_{\mathrm{Ly}}$ towards equatorial regions is much weaker, $\tau_{\mathrm{Ly}} \propto\left(g_{\text {pole }} / g(\theta)\right)^{1.5}$, and even for highest rotational speeds a disk formation becomes impossible as long as the polar wind is not considerably dense (Fig. 4, right panel).

\footnotetext{
11 In a consistent calculation, the von Zeipel theorem has be employed both for the NLTE-
} aspect (occupation numbers) and for the flux-dependent line-acceleration. 
Based on their previous investigations, Curé et al (2005) suggest an alternative possibility to explain the $\mathrm{B}[\mathrm{e}]$ phenomenon. They propose that for near-critical rotation the wind can switch to a slower, shallow-acceleration solution which can lead to a density enhancement and, again in combination with the bi-stability mechanism, to the formation of a dense equatorial disk. From analytic considerations and (1-D) time-dependent hydrodynamics, Madura et al (2007) confirmed most of these results, in particular the slow acceleration for $\Omega>0.85$ and a corresponding increase of density. However, they argue that an inclusion of gravity darkening and 2-D flow effects still renders the formation of dense disks as unlikely.

Let us also note that Zsargó et al (2008b) have identified an additional problem. From 2-D, NLTE calculations by means of their axial-symmetric, NLTE code ASTROROTH they concluded that hydrogen recombination in the disks of $\mathrm{B}[\mathrm{e}]$-supergiants is almost impossible, due to strong ionization from excited levels. Thus, the chance that $\tau_{\mathrm{Ly}}$ becomes much larger than unity and enables line driving by Fe III is rather low, even if the problem of gravity darkening was not present.

On the other hand, Pelupessy et al (2000) solved the momentum equation for sectorial line-driven winds from rotating stars including both the stellar oblateness and gravity darkening, as well as force-multiplier parameters specifically computed for the bi-stability regime. In their computations for stellar parameters far from the bi-stability limit, they found the mass-loss rate and the terminal wind velocity to increase from the equator to the pole (thus confirming the reliability of their approach), however when the counteracting effect of wind bistability was included, they were able to produce a density contrast between the equator and the pole of a factor 10 for a relatively modest rotational velocity of $\Omega=0.6$. One of the attractions of the scenario remains that it may naturally account for the expectation to find outflowing disks only around B-type stars.

From the above discussion it should be evident that more work (in 2-D) is needed to prove whether or not the bistability mechanism is the root cause for the existence of disks around $\mathrm{B}[\mathrm{e}]$-supergiants.

\subsubsection{Winds and magnetic fields}

In contrast to the Sun and other cool stars where magnetic fields are thought to be generated through a dynamo mechanism related to convective motions in the $\mathrm{H} / \mathrm{He}$ recombination zones, hot stars have been considered to be free of strong, dynamo-created surface magnetic fields until recently, because of the absence of strong outer convection zones ( $\mathrm{H}$ remains completely ionized even throughout the photosphere). There are, however, several other possibilities to generate surface magnetic fields, (i) either related to a thin convection zone due to recombination of He III; (ii) due to the strongly convective cores of massive stars which might give rise to dynamo-generated flux tubes diffusing to the surface (Cassinelli and MacGregor 2000, but see MacDonald and Mullan 2004; (iii) by a dynamo that is operating in shear-unstable gas in the radiative stellar envelope (MacDonald and Mullan 2004); (iv) formed already in an early convective phase during stellar formation; (v) or even through compression of interstellar magnetic flux during the initial collapse. It has been shown by Moss (2001), 
Braithwaite and Spruit (2004) and Braithwaite and Nordlund (2006) that a dynamically stable configuration of such fossil fields on long time-scales is actually possible.

Another promising mechanism is the Tayler-Spruit dynamo (Spruit 1999, 2002 and generalizations by Maeder and Meynet 2004; see also Mullan and MacDonald 2005) which does not need a convection zone but is based on a strong instability for generating magnetic fields in radiative layers of differentially rotating stars. This dynamo has been incorporated into recent stellar structure/evolution calculations (Maeder and Meynet 2003a, 2004, 2005, Heger et al 2005, Brott et al 2008) to understand the internal angular momentum transport (though the induced mixing processes might be too strong, e.g., Brott et al 2008). Note, however, that this dynamo is able to create magnetic fields (in the azimuthal direction, of the order of a few $10^{4}$ Gauss $(\mathrm{G})$ ) only outside the stellar core and not in the outermost layers of the star (e.g., Maeder and Meynet 2005).

In recent years, considerable effort has been spent to measure surface magnetic fields, by means of spectropolarimetry (exploiting the circular polarization of the Zeeman components) and a so-called least square deconvolution (Semel 1989, Donati et al 1997) which allows to measure the longitudinal component of the $B$-field averaged over the stellar disk. Though large samples of OB-stars (> 45) have been analyzed (see the material presented by Schnerr 2007 and references therein and Petit et al 2008), significant field strengths above the present detection threshold $(1-\sigma: 40-100 \mathrm{G})$ were detected for only ten stars (excluding chemically peculiar Ap/Bp stars), where in most cases the observations could be fitted by an oblique dipole (but see Donati et al 2006 for the complex magnetic configuration in $\tau$ Sco). Among these ten stars, there are three OB-stars with well known peculiarities in their spectra $\left(\theta^{1}\right.$ Ori C (O4-6V), HD 192612 (O6.5f?peO8fp) and $\tau$ Sco (B0.2V)), with polar field-strengths from 500-1500 G (assuming a dipole), and three $\beta$ Cep stars which incidentally were found to be nitrogen rich (Morel et al 2006). Verdugo et al (2003) report the non-detection of surface magnetic fields in a sample of $12 \mathrm{~A}$-supergiants. Donati et al (2006) conclude that in non-peculiar hot stars, $B$ is weak and/or acts on small scales (spots?).

As should be clear from these introductory remarks, magnetic fields might be large in the stellar interior, but only few "normal" OB-stars show indications of strong surface fields, at least on larger scales. Even weak fields, however, can affect the wind, as we will see in the following. We will report on recent progress on magnetic winds, at first for a simplified case (no rotation) and then for the problem of field aligned rotation and the limiting case of an oblique rotator with very high field-strength.

Magnetically channeled line-driven winds - The confinement parameter. In order to explain the observed X-ray emission from the A0p star IQ Aur, Babel and Montmerle (1997a) suggested that the confinement of the wind by the strong magnetic field leads to a collision from the wind components of the two hemispheres in the closed magnetosphere and thus to the formation of a strong shock. Subsequently, Babel and Montmerle (1997b) used this "magnetically confined wind shock (MCWS) model to explain the periodic variations of the X-ray emission from the O-star $\theta^{1}$ Ori C (see above), requiring a polar field strength at the stellar surface of $B_{*} \approx$ $300 \mathrm{G}$ (present knowledge: $\approx 1100 \mathrm{G}$, Donati et al 2002), where the variability 
should be caused by a circumstellar disk predicted by the MCWS model. In these investigations, a prescribed, fixed magnetic field configuration had been used.

This approximation has been relaxed by ud-Doula and Owocki (2002, see also Owocki and ud-Doula 2004 for a comprehensive analytic description), who investigated the interaction between $B$-fields and winds in detail, based on 2-D time-dependent magneto-hydrodynamics for the simplified case of no rotation and a purely radial line-force. Thus, the external forces in the momentum equation are (effective) gravity, line-acceleration and Lorentz-acceleration, $(4 \pi \rho)^{-1}(\nabla \times \mathbf{B}) \times$ $\mathbf{B}$, and one has to solve for the $B$-field ${ }^{12}$ in parallel with the wind-dynamics. In the following, we will summarize the major findings by ud-Doula and Owocki (2002) and subsequent work.

As it turned out, the most important parameter controlling the structure of the modified wind, $\eta_{*}$ (see below), is related to the ratio of magnetic to wind energy,

$$
\eta(r, \theta)=: \frac{E_{\mathrm{B}}}{E_{\mathrm{wind}}} \approx \frac{B^{2} / 8 \pi}{\rho v^{2} / 2}=\frac{B^{2}\left(R_{*}, \theta\right) R_{*}^{2}}{\dot{M} v_{\infty}} \frac{\left(r / R_{*}\right)^{2-2 q}}{\left(1-R_{*} / r\right)^{\beta}},
$$

assuming a $\beta$-velocity field for the wind and $B(r) \propto\left(R_{*} / r\right)^{q}$ (e.g., $q=3$ for a dipole field). The scaling factor in front of the last expression, evaluated at the magnetic equator under the assumption of a dipole configuration $\left(B_{\mathrm{dipol}}\left(R_{*}, \theta\right)=\right.$ $\left.B_{\mathrm{p}}\left(\cos ^{2} \theta+1 / 4 \sin ^{2} \theta\right)^{1 / 2}\right)$ has been denoted as the "confinement parameter",

$$
\eta_{*}=\frac{B^{2}\left(\theta=90^{\circ}\right) R_{*}^{2}}{\dot{M} v_{\infty}}=\frac{\left(B_{\mathrm{p}} / 2\right)^{2} R_{*}^{2}}{\dot{M} v_{\infty}} \approx 0.19 \frac{B_{100}^{2} R_{10}^{2}}{\dot{M}_{-6} v_{\infty, 8}} .
$$

In the latter expression, the polar field strength is measured in units of $100 \mathrm{G}$, the stellar radius in units of $10 R_{\odot}$, the mass-loss rate in units of $10^{-6} M_{\odot} \mathrm{yr}^{-1}$ and the terminal velocity in units of $1000 \mathrm{~km} \mathrm{~s}^{-1}$.

As we will see below, a value of $\eta_{*}=1$ relates to the onset of larger structural modifications due to $B$. To reach this, e.g., for the prototypical O4(f) supergiant $\zeta \operatorname{Pup}\left(R_{10} \approx 2, \dot{M}_{-6} \approx 4, v_{8}=2\right)$, a field of $B_{\mathrm{p}} \approx 320 \mathrm{G}$ would be required, whereas for the sun (where the magnetic field has a dominating influence on the wind) one finds (with $\dot{M}_{-6} \approx 10^{-8}, v_{8}=0.5, B_{\mathrm{p}} \approx 1 \mathrm{G}$ ) a considerable value for the confinement parameter, $\eta_{*}(\odot) \approx 40$.

For so-called weak winds (Sect. 5], on the other hand, a magnetic field of only $B_{\mathrm{p}} \leq 30 \mathrm{G}$ (well below present detection limits) is needed to reach $\eta_{*}=$ 1 (assuming $\dot{M} \leq 10^{-8} M_{\odot} \mathrm{yr}^{-1}$ and $v_{\infty}=2000 \mathrm{~km} \mathrm{~s}^{-1}$ ). We note that $\tau$ Sco (a prototypical weak-winded star) reaches $\eta_{*} \approx 80$ for the measured field strength of $500 \mathrm{G}$ (Donati et al 2006), i.e., extreme effects are to be expected.

The reason to denote $\eta_{*}$ as a "confinement" parameter relates to the Alfvén radius of the wind. Since MHD waves propagate with the Alfvén speed,

$$
v_{\mathrm{A}}=\frac{B}{(4 \pi \rho)^{1 / 2}} \quad \Rightarrow \quad M_{\mathrm{A}}=\frac{v}{v_{\mathrm{A}}}=\frac{1}{\sqrt{\eta}}
$$

the Alfvén radius can be calculated from the corresponding Mach number $M_{\mathrm{A}}\left(R_{\mathrm{A}}\right)=$ 1, i.e., from the condition $\eta(r, \theta)=1$. For a dipole field, one obtains

$$
R_{\mathrm{A}} \rightarrow R_{*} \quad\left(\eta_{*} \ll 1\right), \quad R_{\mathrm{A}} \approx \eta_{*}^{0.25} R_{*} \quad\left(\eta_{*} \gg 1\right) .
$$

\footnotetext{
$12 \nabla \cdot \mathbf{B}=0$ and $\partial \mathbf{B} / \partial t=\nabla \times(\mathbf{v} \times \mathbf{B})$ in the MHD approximation of infinite conductivity.
} 
Since the Alfvén radius corresponds roughly to the maximum radius of closed loops (see the discussion in ud-Doula and Owocki 2002), the confinement parameter controls this size, i.e., controls whether the wind is confined in such loops.

Wind-structure as a function of $\eta_{*}$. In their calculations, ud-Doula and Owocki (2002) derived the wind-structure from evolving a model with initial conditions of a dipole field superposed upon a spherically symmetric wind. In dependence of the confinement parameter, three exemplary topologies have been found:

(i) For moderately small confinement, $\eta_{*}=0.1$, the surface magnetic fields are extended by the wind into an open, nearly radial configuration. There is still a noticeable global influence from $B$ on the wind, enhancing the density and decreasing the flow speed near the magnetic equator.

(ii) At intermediate confinement, $\eta_{*}=1$, the field lines are still opened by the wind, but retain near the surface a significant tilt which channels the flow towards the magnetic equator. The polar velocity components can become significant, of the order of a few $100 \mathrm{~km} \mathrm{~s}^{-1}$, and a thin disk begins to develop.

(iii) For strong confinement, $\eta_{*}=10$, the field remains closed in loops near the equatorial surface, and the wind is accelerated and channeled upwards from footpoints of opposite polarity. These flows collide near the loop tops, with shock velocity jumps of up to $1000 \mathrm{~km} \mathrm{~s}^{-1}$, leading to hard X-ray emission in the $\mathrm{keV}$ band. These findings are in qualitative agreement with the MCWS model by Babel and Montmerle (1997a), and also in more quantitative agreement with X-ray observations for $\theta^{1}$ Ori C (Gagné et al 2005 and Sect. 6.5.1). Since the shocked material at the loop tops is very dense, its support by magnetic tension becomes unstable, leading to complex infall patterns along the loop lines down to the star. Even for large $\eta_{*}$, the faster radial decline of the magnetic energy (compared to the kinetic energy of the wind) leads to a final dominance of flow effects, and the field lines are extended into an open configuration at larger radii.

Globally, the mass flux of the outer wind increases towards the equator due to the tendency of the field to divert the flow into this direction, whilst the base mass flux in equatorial regions (and beyond for large $\eta_{*}$ ) is lower than the field-free case (see Owocki and ud-Doula 2004 for further details). The terminal velocity, on the other hand, is larger than for a non-magnetic wind at all latitudes except for the equatorial plane, where it becomes significantly reduced due to the higher mass flux. Similar to the case of rotating winds, the total mass flux is barely affected as long a $\eta_{*} \lesssim 10$. For further scaling relations, see ud-Doula et al (2008).

In summary, non-rotating magnetic winds develop a geometrically thin, slow and dense disk, superimposed by a fast, thin polar wind, quite different from rotating non-magnetic winds. Non-radial line forces should be irrelevant since the polar velocities are much larger than in the WCD case. X-rays are to be expected from the channeled flows colliding at loop tops and from shocks neighboring the compressed disk. Finally, it might be speculated whether oblique magnetic rotators can explain part of the observed UV variability (Sect. 6.1) and induce CIRs (Sect. 6.4), because of the large density/velocity contrast which would occur between the magnetic and rotational equator in such stars.

Field aligned rotation and the rigidly rotating magnetosphere model. In a followup study, ud-Doula et al (2008, see also Owocki et al 2005) included rotation 
(aligned with the magnetic field to avoid 3-D calculations) and investigated the question whether magnetic fields could spin up the stellar wind outflow into a "magnetically torqued disk", as suggested by Cassinelli et al (2002). The closed loops present for larger $\eta_{*}$ tend to keep the outflow in rigid body rotation (i.e., $\left.v_{\phi}(r) \propto r\right)$, and it might be possible to propel material into a Keplerian disk if the Alfvén radius is somewhat larger than the Keplerian co-rotation radius, $R_{\mathrm{K}} \approx$ $\left(v_{\text {rot }} / v_{\text {orb }}\right)^{-2 / 3} R_{*}$, where rigid-body rotation would yield a balance between centrifugal and gravitational acceleration. $v_{\text {orb }}=\sqrt{G M / R_{*}}$ is the orbital speed near the equatorial surface 13 Note that strong confinement and rapid rotation are required to obtain $R_{\mathrm{A}}>R_{\mathrm{K}}$. Although various combinations of rotational speeds and magnetic field strengths have been considered, and the analysis has been extended to $\eta_{*}=100$, no stable Keplerian disk has been found to be formed in any of these cases, simply because most of the material does not have the appropriate velocity for a stationary orbit. However, there were clear indications of a "quasi-steady" rigid-body disk, as discussed further below. Note also that in the opposite case of slow rotation (more precisely, for $R_{\mathrm{A}}<R_{\mathrm{K}}$ ), the basic results as obtained for the non-rotating case remain valid.

For the limiting case of very high field strength, $\eta_{*} \rightarrow \infty$, Townsend and Owocki (2005) developed the alternative "rigidly rotating magnetosphere" model, originally designed to investigate the prototypical Bp star $\sigma$ Ori $\mathrm{E}$ with $B \sim 10^{4} \mathrm{G}$. In this model, the field lines behave like rigid tubes, and the outflowing material is constrained along corresponding trajectories fixed by the prescribed field geometry (see also the rigid field hydrodynamics approach by Townsend et al 2007).

For sufficient rotation then, minima in the effective potential along each field line should gradually fill with plasma, forming an almost steady, co-rotating magnetosphere. For the oblique rotator $\sigma$ Ori E, this should occur in the form of two co-rotating clouds. Time-series of synthetic $\mathrm{H}_{\alpha}$ profiles for such a geometry are consistent with the observed behaviour (Townsend et al 2005). As mentioned above, the time-dependent calculations for large $\eta_{*}$ by ud-Doula et al (2008) have confirmed the basic picture of a rigid-body disk (for aligned rotation), but with a complex dynamic behaviour with infall and outflow limiting the growth of the disk. E.g., the eventual centrifugal breakout of such material might suggest a new heating mechanism (via magnetic reconnection) to explain the hard X-ray flares, as observed in Bp-stars (Groote and Schmitt 2004) and simulated by ud-Doula et al (2006).

\section{Stationary models of radiation driven winds}

\subsection{Predictions from the improved CAK approach}

As was noted earlier, the original CAK approach to compute the wind structure has been massively improved (Friend and Abbott 1986, Pauldrach et al 1986, Pauldrach 1987, Pauldrach et al 1994, 2001). Whilst the CAK models were computed in local thermodynamical equilibrium (LTE), departures from LTE have been included because of the strong influence of the radiation field and the low

\footnotetext{
13 This approximation for $R_{\mathrm{K}}$ assumes the star to be far away from the $\Omega \Gamma$ limit.
} 
densities in the atmospheres of hot massive stars. Another significant improvement involved the inclusion of millions of lines (predominantly from iron-group elements) from some 150 different ions - in contrast to the single C III list used in the original work of CAK. Within the current state-of-the-art modified CAK modeling, the rate equations, radiative transfer equations, energy equation, and approximate hydrodynamic equations, using force-multiplier parameters, are all accounted for. More simplified models relying on an approximate solution for the rate-equations and an analytic approach to solve the hydrodynamic equations (with consistent, depth dependent force-multiplier parameters) have been presented by Kudritzki (2002).

A complementary approach currently in use concerns the hydrodynamical method of Krtička (2006). As in the models by Pauldrach and co-workers ("WMbasic", see Sect.4.2.1), the various equations are consistently solved, but the concept of force multipliers is dropped, though the Sobolev approximation is still applied. The major advantage of this method is that it allows for a multi-component description of the hydrodynamical equations, which enables an investigation of potential metal-ion decoupling from the $\mathrm{H} / \mathrm{He}$ plasma. A drawback of the Krtička (2006) models is that line-blocking and multi-line effects are not accounted for limiting their applicability to weaker winds.

\subsection{Predictions using a Monte Carlo radiative transfer approach}

A different approach involves the "Monte Carlo" method developed by Abbott and Lucy (1985), where photons are emitted at an inner boundary, and their scattering history is tracked on their journey outwards. At every interaction some amount of momentum and energy is transferred from the photon to the ion under consideration. From a macroscopic point of view, the overall integrated mass-loss rate is obtained from global energy conservation:

$$
\frac{1}{2} \dot{M}\left(v_{\infty}^{2}+v_{\mathrm{esc}}^{2}\right)=\Delta L
$$

where $\Delta L$ is the total amount of energy that has been transferred per second from the radiation field to the scattering ions in the wind. The main drawback of this approach is that one first needs to establish an appropriate velocity law, $v(r)$, generally using an empirically pre-determined $v_{\infty}$, although in principle it is possible to iterate and obtain dynamically consistent mass-loss rates (see Springmann and Puls 1998, Vink et al 1999).

One of the major advantages of the Monte Carlo method is that it easily allows for multi-line scattering, which becomes important in denser winds, as already outlined by Friend and Castor (1983, but see also Puls 1987). Before the year 2000, theoretical mass-loss rates fell short of the observed rates for dense $\mathrm{O}$ star and WR winds, whilst for weak winds the oft-used single line approach overestimated the mass-loss rates considerably. Although early Monte Carlo simulations (e.g. Abbott and Lucy 1985) only treated line scattering, this approximation has been relaxed in the mass-loss rate determinations by Vink et al (2000, 2001), although further improvements to the method, e.g., in terms of line branching (Sim 2004) and wind clumping need to be considered in future model generations. 
Here we wish to mention that the high mass-loss rates derived for dense winds when accounting for multi-line scattering (as in the Monte Carlo method) do not violate the principle of momentum conservation. An appealing picture is that of "hemispheric scattering" where a single photon can be launched in one direction, transfer its momentum to an ion, and travels towards the opposite hemisphere repeating the same process over and over again, thereby pumping up the momentum transfer in the wind. One may note that all what really happens is that at each iteration the photon is slightly red-shifted, until it eventually runs out of energy. In reality, we know that hemispheric scattering will not occur. It has been demonstrated (e.g., Springmann 1994, Gayley et al 1995) that when one employs realistic line lists, photons follow paths that are less extreme, but the essential point is that multiply scattered photons are always adding radially outward momentum to the wind, and the wind momentum may easily exceed the so-called single-scattering limit, i.e., $\dot{M} v_{\infty} /(L / c)$ can become larger than unity.

Vink et al (2000, 2001) used the Monte Carlo method over a wide range of stellar parameters and derived a "mass-loss recipe" using multiple linear regressions to their model results. On the hot side of the predicted bi-stability jump at $\sim 25000 \mathrm{~K}$ (but see Sect.4.2.1), the rates of OB supergiants should roughly scale as

$$
\dot{M} \propto L^{2.2} M^{-1.3} T_{\text {eff }}\left(v_{\infty} / v_{\text {esc }}\right)^{-1.3} .
$$

when $L$ is measured in $10^{5} L_{\odot}, M$ in $30 M_{\odot}$ and $T_{\text {eff }}$ in $40 \mathrm{kK}$. This relation predicts that the mass-loss rates should strongly depend on the luminosity $\left(L^{2.2}\right)$, steeper than $\dot{M} \propto L^{1.6}$ which is often quoted and relies on the simplified scaling relation Eq. 114 with $\alpha^{\prime} \approx 0.6$. The reason is that the efficiency of multi-line effects accounted for in Monte Carlo simulations increases with increasing wind density, i.e., for more luminous stars, whereas in (modified) CAK models these effects are normally neglected, particularly in the scaling relations presented in Sect.2.1. Because the mass-loss rate also scales with stellar mass as $M^{-1.3}$, and applying a typical $M-L$ ratio of $L \propto M^{x}$ with $x=2$ - 3 for massive stars, one obtains an overall $\dot{M}$ scaling as $L^{1.55-1.77}$, in agreement with observational findings.

The basic success of the Monte Carlo method is exemplified when comparing Figs. 1 and 3 from Vink (2006), which display the degree of agreement between modified CAK models and observations on the one side and Monte Carlo models and observations on the other: by properly including multiple scatterings in the predictions, the results are equally successful for relatively weak (with $\dot{M} \sim 10^{-7}$ $M_{\odot} \mathrm{yr}^{-1}$ ) as well as denser winds (with $\dot{M} \sim 10^{-5} M_{\odot} \mathrm{yr}^{-1}$ ). The predictions can be conveniently expressed via the WLR. For O-stars hotter than $27500 \mathrm{~K}$, the relation is shown in Fig. 5 and given by Eq. (16) with $x=1.83$, corresponding to $\alpha^{\prime}=0.55$. For objects on the cool side of the bi-stability jump, the mass-loss rate increases and the slope of the WLR becomes $x=1.91$, corresponding to $\alpha^{\prime}$ $=0.52$.

Monte Carlo predictions for hot stars of lower mass were computed by Vink and Cassisi (2002), whilst modified CAK predictions for Central Stars of Planetary Nebulae were computed by Pauldrach et al. (1988, 2004), see page 51. 


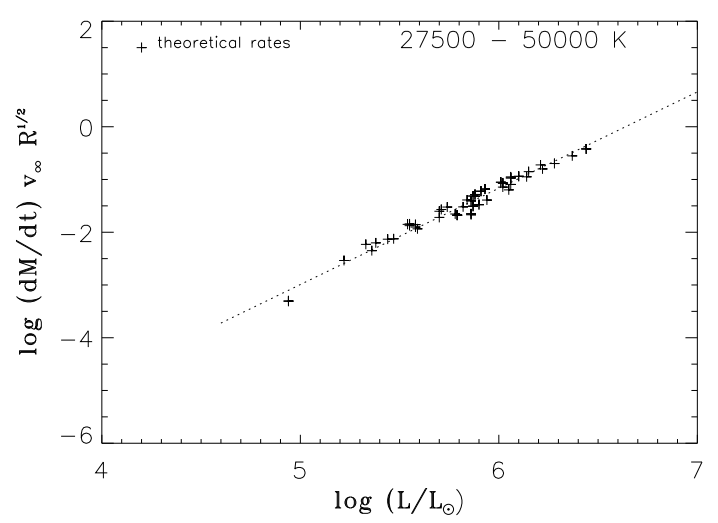

Fig. 5 Predicted WLR for O stars hotter than $27 \mathrm{kK}$ for a range of $(L, M)$-combinations in the upper HRD. From Vink et al (2000).

\subsection{Predictions for models close to the Eddington limit}

The predictions of Eq. (37) are valid for models that are located at a sufficient distance from the Eddington limit, with $\Gamma \leq 0.5$. There are two regimes where this is no longer the case: (i) stars that have formed with large initial masses and luminosities, i.e. very massive stars (VMS) with $M \gtrsim 100 M_{\odot}$, and (ii) less extremely luminous "normal" stars that approach the Eddington limit when they have lost a substantial fraction of their initial mass. Examples of such objects are the LBVs and the more common evolved WR stars.

Vink and de Koter (2002) and Smith et al (2004) showed with their Monte Carlo computations that when lowering the mass for constant luminosity, the mass-loss rate increases more rapidly than Eq. (37) indicates. For these LBV models, $\dot{M} \propto M^{-1.8}$ instead of $M^{-1.3}$ as for normal OB supergiants. In other words, when stars start to lose mass, not only does the mass-loss rate increase due to a lower stellar mass, but as the mass-loss rate increases more rapidly this leads to a strong positive feedback.

An illustration of this feedback-effect for VMS in the mass range 100-300 $M_{\odot}$ was provided by Vink (2006) where it was noted that VMS mass loss drastically increases when the objects approach the Eddington limit. Vink and de Koter (2005) also simulated mass loss close to the Eddington limit when performing a pilot study for evolved late-type WR (WN and WC) winds, as a function of metallicity. They showed that these mass-loss rates were a factor 6-7 higher than using the Vink et al (2000) recipe for OB supergiants - for objects with similar stellar parameters, as a result of the closer proximity to the Eddington limit.

A different approach to the problem of winds close to the Eddington limit was employed by Gräfener and Hamann (2008). One should note that their WR windmodels were not applied to evolved WR stars, but to WR stars in which the broad emission lines form due to a large intrinsic luminosity. For WR wind-models, the 


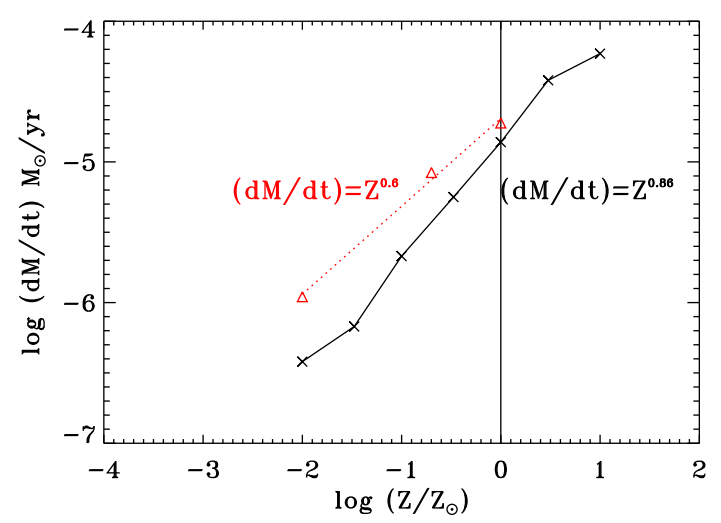

Fig. 6 Mass-loss predictions as a function of $Z$, for objects with $Z / Z_{\odot}>1 / 100$. The solid line indicates the $\dot{M}(Z)$ dependence from Monte Carlo simulations by Vink et al (2001) and the dotted line corresponds to the predictions by Kudritzki (2002) . See text.

CAK formalism is no longer applicable, as a myriad of weak iron lines provide massive line overlap, and the wind has become optically thick.

The framework of optically thick winds has been employed in studies of Kato (1985) and Nugis and Lamers (2002) where the basic assumption is that the critical point of the problem is the sonic point, similar to recent suggestions by Lucy (2007a b) regarding "normal" O-star wind $\$$, and that the corresponding conditions can be described in the diffusion limit. In the transonic region, the Rosseland mean opacity shows an outwards increasing behaviour and, through the use of the equation of continuity, $\dot{M}$ follows from the specific conditions at the sonic point. In the more advanced studies of Gräfener and Hamann (2005, 2008) the momentum equation of the entire wind is accounted for and a strong dependence of $\dot{M}$ on the proximity to the Eddington limit is also predicted for these models (see also Sect.4.2.3.

\subsection{Predictions for very low $\mathrm{Z}$ and Pop III stars}

\subsubsection{The observable massive stars at low $Z$}

Vink et al (2001) applied their Monte Carlo models to OB-stars within a Z-range representative for the observable Universe, $Z / Z_{\odot}>1 / 100$. The corresponding results are compared to those from Kudritzki (2002) in Fig. 6. Over the last decades the modified CAK models predicted $\dot{M} \propto Z^{m}$ with $m$ in the range 0.47-0.94 (Abbott 1982, Kudritzki et al 1987, Kudritzki 2002). The main reason for this scaling is that the CAK $k$ parameter is strongly $Z$-dependent, whilst second order effects that relate to how $\alpha$ varies with $Z$ have a larger effect on the terminal wind velocity instead (see pages 11ff.).

\footnotetext{
14 A discussion of this assumption is beyond the scope of this review.
} 
As can be noted from the figure, the Vink et al (2001) predictions exhibit a steeper slope $(m=0.85)$ than the Kudritzki (2002) predictions, with $m=0.5-0.6$. Again, the reasons are thought to be related to the neglect of multi-scattering in CAK-type models, i.e., the corresponding single-line approach could result in both an $\dot{M}-L$ dependence and an $\dot{M}-Z$ dependence that are too shallow. On the other hand, most results computed using the Monte-Carlo method have not accounted for a $v_{\infty}-Z$ dependence, which is anticipated to result in an overestimate of $m$. Therefore, a value of $m=0.7$ has been recommended as the appropriate value for the mass-loss scaling with $Z$ for OB-stars (Vink et al 2001, Krtička 2006). The observed metallicity dependence of OB-stars will be discussed in Sect.4.2.1.

\subsubsection{Objects at very low $Z$ and Pop III stars}

Extending the predictions to very low $Z / Z_{\odot} \lesssim 10^{-2}$, where stars can no longer be observed individually, it has been shown that $\dot{M}$ keeps dropping until the winds reach a regime where they become susceptible to ion-decoupling and multi-component effects (Krtička et al 2003). The only way to maintain a one-fluid wind model is through an increase of the Eddington factor by pumping up the stellar mass and luminosity. Such an increase in stellar mass is by no means artificial, as the earliest generations of massive stars are anticipated to be more massive than today's population since stars formed with fewer metals to provide the cooling at the very least resulting in a larger Jeans mass.

In case of Pop III stars with truly "zero" metallicity, i.e. only $\mathrm{H}$ and He present in the atmosphere, it seems unlikely that these stars would develop line-driven winds of significant strength (Kudritzki 2002, Krtička and Kubat 2006). Nonetheless, other effects may contribute to the driving. Interesting possibilities include stellar rotation and pulsations, although pure vibration models for Pop III stars also indicate little mass loss via pulsations alone (Baraffe et al 2001). Perhaps a combination of several effects would result in large mass loss close to the Eddington limit. Moreover, we know that even in the present-day Universe a significant amount of mass is lost in LBV type eruptions (potentially driven by continuum radiation pressure, see page (56) which might be also relevant for the First Stars (Vink and de Koter 2005, Smith and Owocki 2006).

\subsubsection{Winds enhanced by self-enrichment}

Given that the First Stars are potentially massive, luminous, and rapidly rotating, it is not inconceivable that, despite the fact that the first generation(s) of massive stars start their evolutionary clock with few metals, the objects may enrich their outer atmospheres with nitrogen and carbon due to rotational mixing (Mevnet et al 2006), inducing a strong line-driven wind (Vink 2006, Gräfener and Hamann 2008).

In a first attempt to investigate the effects of self-enrichment on the total wind strength, Vink and de Koter (2005) performed a pilot study of WR mass loss versus $Z$. The prime interest in WR stars here is that these objects, especially those of WC subtype, show the products of core burning in their outer atmospheres.

Although the last few decades provided increasing evidence that WR winds are radiatively-driven (Lucv and Abbott 1993, Gavlev et al 1995, Springmann and Puls 1998, Nugis and Lamers 2002, Gräfener and Hamann 2005), the question of a WR 


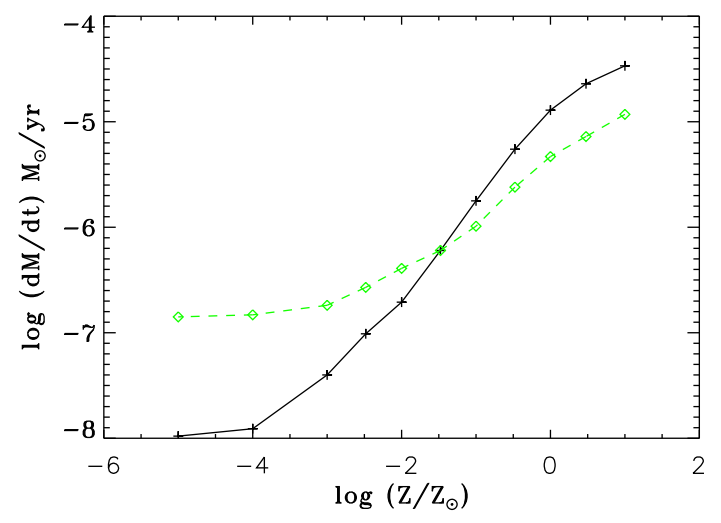

Fig. 7 Monte Carlo WR mass-loss predictions as a function of $Z$. The dark line represents the late-type WN stars, whilst the lighter dashed line shows the results for late-type WC stars. The slope for the WN models is similar to the predictions for OB-supergiants, whilst the slope is shallower for WC stars. At low $Z$, the slope becomes smaller, whilst it flattens off entirely at $Z / Z_{\odot}=10^{-3}$. The computations are from Vink and de Koter (2005).

$Z$-dependence remained controversial, with some stellar modelers extrapolating $Z$ dependencies from $\mathrm{O}$ stars, whilst others assumed WR winds to be $Z$-independent.

The reasoning behind the assertion that WR winds may not be $Z$-dependent was that WR stars enrich themselves by burning $\mathrm{He}$ into $\mathrm{C}$, and it could be the large $\mathrm{C}$-abundance that is the most relevant ion for the $\mathrm{WC}$ wind driving, rather than the sheer number of $\mathrm{Fe}$ lines. Figure 7 shows that despite the fact that the $\mathrm{C}$ ions overwhelm the amount of Fe, both late-type WN (dark line) and WC (light line) show a strong $\dot{M}-Z$ dependence, basically because Fe has such a complex electronic structure.

The implications of Fig.7 7are two-fold. The prediction that mass loss no longer decreases when $Z / Z_{\odot}$ drops below $\sim 10^{-3}$ (due to the final dominance of driving by carbon lines) suggests that once massive stars enrich their outer atmospheres, radiation-driven winds might still exist, even if the object started its life with negligible amounts of metals. Whether the rate of mass loss is high enough to seriously alter the evolutionary tracks of the First Stars also depends on other physical factors, such as the proximity to the Eddington- or the $\Omega \Gamma$-limit.

The second point to address regarding Fig. 7 is the new result that the massloss rates of WR stars drop steeply with $Z$ at subsolar $Z$. This is anticipated to have important consequences for black hole formation and the progenitor evolution of long duration gamma-ray bursts. The collapsar model of Woosley (1993) requires a rapidly rotating core before collapse, but at solar $Z$ stellar winds are anticipated to remove significant angular momentum (Zahn 1992). The WR $\dot{M}-Z$ dependence from Fig. 7 provides the intriguing possibility to maintain rapid rotation because WR winds are predicted to be weaker at lower $Z$.

Interestingly, the WR $\dot{M}-Z$ dependence also appears to be able to reproduce the drop in the WC/WN ratio at low $Z$ (Eldridge and Vink 2006). Although there are various reasons to assume that additional effects such as rotational mixing and 
binarity play an important role for the formation of WR stars, the results suggest that the mass-loss- $Z$ dependence is a first order effect as far as the WC/WN ratio is concerned.

\section{Observed wind parameters}

As we have seen in the preceeding sections, radiation driven wind theory provides precise predictions for the two global parameters of hot stellar winds, namely the terminal velocity, $v_{\infty}$, and the mass-loss rate, $\dot{M}$, as a function of stellar parameters (including abundances, rotation rates, magnetic field-strengths). The most prominent prediction concerns the presence of a wind-momentum luminosity relation (if "contaminating" effects do not dominate), which should allow for an easy check of the theory when the global wind parameters are known from observations.

Later on, we will summarize recent results on such comparisons. In order to do so, we need to know how and to which precision these wind parameters can be "measured".

A few caveats. Most of the following investigations (still) rely on the assumptions of the standard model (Sect.2.1), partly extended to allow for the presence of inhomogeneities (clumping) and X-rays (see below). Within this model, the massloss rate follows from the continuity equation (Eq. 1), and practically all diagnostics base on the approach that certain observational features (spectra or SEDs) are fitted by corresponding radiative transfer calculations on top of a wind model with a density expressed in terms of the mass-loss rate, $\rho(r)=\dot{M} /\left(4 \pi r^{2} v(r)\right)$, and a $\beta$-velocity field,

$$
v(r)=v_{\infty}\left(1-b \frac{R_{*}}{r}\right)^{\beta} .
$$

Remember that this functional form relies on the theoretical predictions (Eq. 12). The constant $b$ fixes the onset of the wind, usually obtained from requiring a smooth transition between photospheric density-/velocity-stratification and wind conditions, or by imposing a certain minimum velocity $v\left(R_{*}\right)=v_{\min }$, of the order of the isothermal speed of sound.

By varying $\dot{M}, v_{\infty}$ and $\beta$, an optimum fit is aimed at and the so-called "observed wind parameters" are just the outcome of such fitting procedures. Insofar, already their determination relies on a consistent description of the wind and the photosphere, since not only the wind properties but also additional atmospheric conditions such as $T_{\text {eff }}, \log g$ and abundances affect the radiative processes which are modeled to fit the observations. Whenever "observed wind parameters" are presented, one should be aware of the fact that already these are the result of diagnostic techniques based on a substantial amount of theoretical modeling. Depending on the degree of sophistication of the underlying model but also on the part of the spectrum and the specific process which is used for the determination, the reliability of the results can vary drastically.

Even worse, by investigating the scaling properties of particular solutions, it turns out that such fits are not unique, but depend, subject to the considered process, on certain combinations of wind parameters and stellar radius. Important quantities are the "optical depth invariants" $Q_{\text {res }}$ for resonance lines with a line 
opacity $\propto \rho$ and $Q$ for recombination based line processes (such as $\mathrm{H}_{\alpha}$ ) with opacities $\propto \rho^{2}$,

$$
Q_{\mathrm{res}}=\frac{\dot{M}}{R_{*} v_{\infty}^{2}}, \quad Q=\frac{\dot{M}}{\left(R_{*} v_{\infty}\right)^{1.5}}
$$

(details are given in the following). Individual mass-loss rates can be obtained only when the terminal velocity and the stellar radius (depending on distance which is problematic for Galactic objects) are known, or, at least in principle, if resonance and recombination processes are fitted simultaneously by means of NLTE atmosphere codes. Otherwise, the result of such fits is not the mass-loss rate but one of the above scaling invariants. Thus, even if the fit looks perfectly, the same needs not to be true for the derived mass-loss rate.

The standard model is certainly an (over?)-simplification. Significant evidence for deviations from spherical symmetry, non-stationarity, clumpiness and shocks has been found from the beginning of wind observations on. Aspects related to rotation and magnetic fields have already been discussed in Sect. 2.2. and we will turn to problems related to time-dependence and inhomogeneity in Sect. 6 . Though these phenomena had been widely ignored with respect to diagnostical methods until the late 1990's, increasing observational and computational feasibilities have lead to the conclusion that certain aspects must be included to avoid obvious inconsistencies. In due course, simplified approaches have been developed to account for these aspects, where the inclusion of wind-clumping and the X-ray/EUV emission from shocked material into the present generation of NLTE codes are the most prominent examples of such updates. Note, e.g., that the optical depth invariant $Q$ as defined in Eq. 39 needs to be modified with respect to the socalled clumping factor if the winds are considered as inhomogeneous (Sect. 6.3, Eq. 54).

Obviously, a detailed inclusion of deviations from the standard model requires significant effort regarding both diagnostics and the development of new models and methods, a task which is just at the beginning to date. Examples are the developments of multi-dimensional NLTE codes such as ASTROROTH (Georgiev et al 2006) mentioned already in the context of B[e] stars (Sect. 2.2.1) and a 2-D description of rotational effects within the spectrum synthesis based on NLTE model atmospheres, as reported by Bouret et al (2008). Further progress can be expected in the near future.

\subsection{Diagnostic methods}

Diagnostic methods can be roughly divided into two different classes. Approximate methods concentrate on the analysis of one specific process and try to deduce specific (wind-)parameters by a simplified description, usually by considering the wind physics alone and requiring additional information from external photospheric diagnostics.

Alternatively, modern diagnostics rely on the use of "unified" NLTE model atmospheres. Stellar and wind parameters are derived simultaneously by optimizing synthetic spectra and SEDs for a large wavelength range, comprising diagnostics of a multitude of processes with different dependencies and scaling relations. 
In order to draw correct conclusions, these processes have still to be understood though.

\subsubsection{Approximate methods}

A detailed discussion and comparison of the various approximate methods to derive global wind parameters has been given by Kudritzki and Puls (2000), and we will summarize only important aspects.

The two most prominent line types formed in a stellar wind are P Cygni profiles with a blue absorption trough and a red emission peak, and pure emission profiles (or absorption lines refilled by wind-emission). Their different line shapes are caused by different population mechanisms of the upper (emitting) energy level of the transition.

In a P Cygni line, the upper level is populated by the interplay between absorption from and spontaneous decay to the lower level, usually the ground state of an ion. This process is called line-scattering. If, on the other hand, the upper level is populated via independent processes, e.g., by recombination into this level or decay from levels above, a pure emission line (or an absorption line weaker than formed by purely photospheric processes) will result. The former line type as seen in the (F)UV is majorly used to determine the velocity field in the wind, particularly $v_{\infty}$, and the latter $\left(\mathrm{H}_{\alpha}\right.$, but also He II $\left.4686, \mathrm{Br}_{\alpha}\right)$ to derive $\dot{M}$, or, more precisely, $Q$.

Analysis of UV P Cygni profiles. (F)UV P-Cygni lines from hot stars (e.g., C IV, $\mathrm{N}$ v, Si IV, O VI, P V) are usually analyzed by means of the so-called "SEI" method (Sobolev plus exact integration, see Lamers et al 1987), based on a suggestion by Hamann (1981a). The central quantity which controls the line formation process is the (radial) Sobolev optical depth of the specified line (cf. page 7 )

$$
\tau_{\mathrm{Sob}}(r)=\frac{\bar{\chi}(r) \lambda}{\mathrm{d} v / \mathrm{d} r} \frac{R_{*}}{v_{\infty}}, \quad \bar{\chi}(r)=\frac{\pi e}{m_{\mathrm{e}} c} f n_{1}(r) .
$$

$f$ is the oscillator-strength and $n_{1}$ the lower occupation number of the transition, neglecting stimulated emission. Here and in the following, radii $r$ and velocities $v$ are measured in units of the stellar radius and the terminal velocity, respectively (remember that the line optical depth in a stellar wind is an almost purely local quantity, cf. page 7). Relating the occupation number, $n_{1}$, to the local density, this quantity can be expressed by

$$
\tau_{\mathrm{Sob}}(r)=\frac{k(r)}{r^{2} v \mathrm{~d} v / \mathrm{d} r}, k(r)=E(r) q(r) \frac{\dot{M}}{R_{*} \nu_{\infty}^{2}} \frac{\left(\pi e^{2}\right) /\left(m_{\mathrm{e}} c\right)}{4 \pi m_{\mathrm{H}}} \frac{A_{\mathrm{k}}}{1+4 Y_{\mathrm{He}}} f \lambda
$$

where $E$ is the excitation factor of the lower level ( $\equiv 1$ for resonance lines coupled to the ground-state), $q$ the ionization fraction, $A_{\mathrm{k}}$ the abundance of the element with respect to hydrogen and $Y_{\mathrm{He}}$ the helium abundance. Obviously, this expression is scaling invariant with respect to the quantity $Q_{\text {res }}=\dot{M} /\left(R_{*} v_{\infty}{ }^{2}\right)$ (cf. Eq. 39) as long as the ground-state population of the considered ion is proportional to $\rho$ throughout the wind, e.g., if the ion is a major one. From the latter equation and 

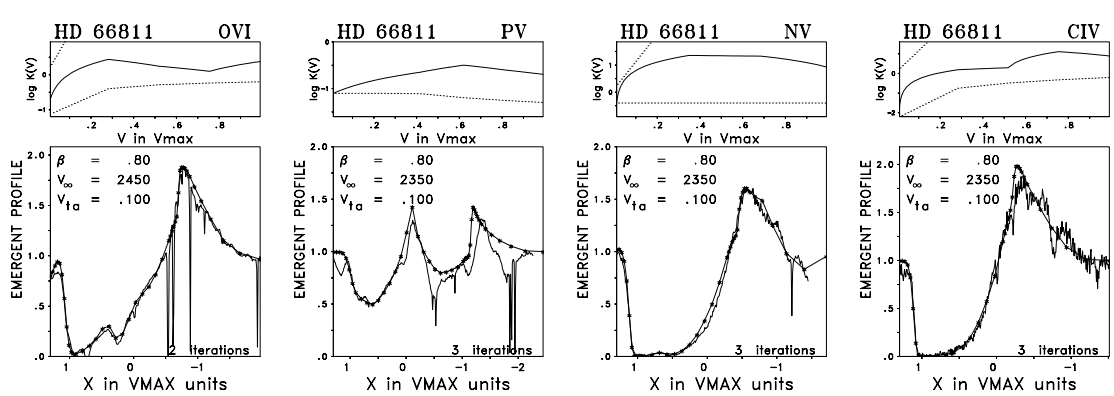

Fig. 8 SEI fits to the P Cygni profiles of O VI, P V, N V and C IV of $\zeta$ Pup (O4I(f)) as observed by COPERNICUS (Morton and Underhill 1977). An almost unique solution with $v_{\infty}=2350 \mathrm{~km} \mathrm{~s}^{-1}$ and $\beta=0.8$ is found for all four profiles, with a "micro-turbulence" of $10 \%$ of $v_{\infty}$. The upper panels show the derived run of the quantity $k(v) \propto \dot{M} q$ (Eq. 41). Note the black troughs in N v and C IV. From Haser (1995).

the assumed $\beta$-velocity law, it should be clear that the maximum set of deducible parameters from a fit to the observations consists of $\left(v_{\infty}, \beta, k(r)\right)$, see Fig. 8

Thus, information on the mass-loss rate is hidden in the quantity $k(r)$, and mass-loss rates can be derived from the P Cygni profiles of resonance lines (if at all) only when external knowledge about ionization, abundance and stellar radius is available. Exemplarily, Howarth and Prinja (1989) and Haser (1995) inferred the product $\dot{M}\langle q\rangle$ for large O-star samples from unsaturated profiles, where $\langle q\rangle$ is a suitable (spatial) average of the corresponding ionization fraction.

Most of the "strategic" P Cygni lines, however, are saturated under typical conditions ( $\mathrm{C} \mathrm{IV}, \mathrm{N}$ V in early O-stars and Si IV in late O-supergiants). In these cases, the derivation of mass-loss rates (or more precisely, of the product $\dot{M}\langle q\rangle$ ) becomes impossible, and only lower limits are accessible. The reason that these lines are often saturated has to do with the particular dependence of the line optical depth on the product $\left(r^{2} v \mathrm{~d} v / \mathrm{d} r\right)^{-1}$, which, as already stated on page 10, varies according to $v(r)^{1 / \beta-2}$. For lines with a rather constant ionization fraction throughout the wind and typical O-star parameters, this variation is only mild, and a line most likely remains optically thick throughout the wind (i.e., the P Cygni profile is saturated) when it has a large optical depth already in the sonic region. In this case, "only" terminal velocities (from the frequency position of the blue absorption edge) and velocity field parameters $\beta$ (from the shape of the emission peak) can be measured (e.g., Howarth and Prinja 1989, Groenewegen et al 1989 and Haser 1995). Typical values $\beta=0.7 \ldots 1.5$ have been found for OB-stars, where supergiants show a clear tendency towards higher $\beta$. For A-supergiants, values as high as $\beta=3 \ldots 4$ have been derived (e.g., Stahl et al 1991, Kudritzki et al 1999).

The most severe problem of P Cygni line diagnostics is related to the presence of so-called black troughs, which are extended regions in the absorption part of saturated profiles with almost zero residual intensity (see Fig. 8). These troughs cannot be explained without further assumptions. Hamann (1981a b) introduced a highly supersonic "microturbulence" (of the order of $10 \%$ of $v_{\infty}$ ) throughout the entire wind to overcome this and related problems, Since in this case the intrinsic absorption profile is extremely broad, the observed troughs can be simulated, in 
parallel with the red-shifted emission peaks (see also Groenewegen and Lamers 1989 and Haser 1995 for the inclusion of a depth-dependent velocity dispersion).

Lucy (1982, 1983) suggested an alternative, more physical explanation for the generation of black troughs due to enhanced back-scattering in multiply nonmonotonic velocity fields, which most likely are present according to observations and time-dependent hydrodynamical simulations (see Sect. 6). Detailed UV line formation calculations have shown that black troughs can be actually created in the profiles of such models (Puls et al 1993a, Owocki 1994b).

Note finally that until very recently UV resonance lines have been considered as being (almost) uncontaminated by clumping effects. As we will see in Sect.6.3, this might not be true, due to the effects of porosity or "vorosity" (= velocity porosity).

$H_{\alpha}$ diagnostics. To avoid the problems inherent to the $\dot{M}$-determination from UV P Cygni lines (which, moreover, require space-bound observations), the commonly accepted standard diagnostics to derive $\dot{M}$ relies on the wind emission in $\mathrm{H}_{\alpha}$, where the influence of ionization (which is almost complete) and abundance becomes almost negligible. The idea to use $\mathrm{H}_{\alpha}$ as a mass-loss indicator goes back to Klein and Castor (1978), and Leitherer (1988), Drew (1990), Gabler et al (1990), Scuderi et al (1992) and Lamers and Leitherer (1993) applied this method to derive first results for a variety of hot stars. Puls et al (1996) extended the method to obtain a fast analysis tool and to eliminate some systematic errors inherent to previous approaches.

The basic difference between $\mathrm{H}_{\alpha}$ (and similarly behaving lines such as He II 4686 and $\mathrm{Br}_{\alpha}$ ) and $\mathrm{P}$-Cygni lines is related to the source function, which in the former case is rather constant throughout the wind, as long as the involved levels are predominantly fed by recombinations or decays from upper levels. This condition is met in O- and early B-stars. For cooler stars, the population by pumping due to Lyman lines becomes equally important, until, in the A-supergiant domain, these lines and the Lyman continuum become optically thick and the corresponding transitions reach detailed balance. Consequently, the second level of hydrogen $\left(n_{2}\right.$, the lower level of $\left.\mathrm{H}_{\alpha}\right)$ becomes the effective ground state of $\mathrm{HI}$ and the line behaves as a scattering line, thus displaying a typical P-Cygni signature when the winds are sufficiently strong. In such cases, the profile is usually visible until a blue edge frequency corresponding to $v_{\infty}$, which allows to derive also this quantity and thus enables a complete wind analysis in the optical alone (see Kudritzki et al 1999).

Since neutral hydrogen is almost always a trace ion in the winds of OBA stars, the opacity of $\mathrm{H}_{\alpha}$ scales with $\rho^{2}$,

$$
\bar{\chi}(r) \propto b_{2}(r)\left(\frac{\dot{M}}{r^{2} v(r)}\right)^{2}, \quad \tau_{\mathrm{Sob}}(r)=\frac{\bar{\chi}(r) \lambda}{\mathrm{d} v / \mathrm{d} r} \frac{R_{*}}{v_{\infty}} \propto \frac{\dot{M}^{2}}{\left(R_{*} v_{\infty}\right)^{3}} \frac{b_{2}(r)}{r^{4} v^{2} \mathrm{~d} v / \mathrm{d} r},
$$

i.e., the scaling invariant is $Q^{2}$ (Eq. 39), and $b_{2}$ is the NLTE departure coefficient of $n_{2}$ which can be rather easily calculated 15 Although similar arguments hold for

15 When $n_{2}$ becomes the effective ground state, the opacity scales with $\rho^{2} / W$ with dilution factor $W$, see Kudritzki and Puls 2000. 
the formation of He II 4686, the corresponding departure coefficients have a more complicated (and more uncertain) stratification which favors the use of $\mathrm{H}_{\alpha}$.

Scaling relations for the equivalent width of $\mathrm{H}_{\alpha}$ in O-stars have been provided by Puls et al (1996), and typical errors for $Q$ are of the order of $30 \%$, where these errors can reach values below $10 \%$ for strong wind emission. Also here, the velocity field parameter $\beta$ can (and has to) be determined from the shape of the emission peak or wing, resulting in typical values $\beta \approx 1$ for O-type supergiants (Puls et al 1996). For weaker winds, on the other hand, the wind emission is low and "hidden" within the (rotationally broadened) photospheric profile, and accurate determinations of $\dot{M}$ become more difficult. Detailed NLTE calculations based on unified model atmospheres (Sect.4.1.2) are required to derive meaningful results in such cases. But even if doing so, uncertainties up to a factor of two in $\dot{M}$ can arise if the photospheric absorption profile is only marginally refilled. Such uncertainties are rooted in the strong dependence of the mass-loss rate on $\beta$ and the fact that this parameter can no longer be derived (because of the hidden wind emission) but has to be assumed instead (see, e.g., the corresponding error analysis in Repolust et al 2004).

At even lower wind-densities (roughly below a few times $10^{-8} M_{\odot} \mathrm{yr}^{-1}$ in the case of O-/early B-stars), $\mathrm{H}_{\alpha}$ completely looses its sensitivity to mass loss, due to a vanishing wind emission. For such low $\dot{M}$ (which is present, e.g, in many SMC O-stars and so-called weak-winded stars, see Sect. 5), other diagnostics must be used, either UV resonance lines or $\mathrm{Br}_{\alpha}$ (see below).

The major problem of $\mathrm{H}_{\alpha}$, however, is its $\rho^{2}$ dependence. Any significant degree of clumping will lead to an overestimate of the mass-loss rates if this is neglected in the analysis. Somewhat advantageous, however, is the fact that $\mathrm{H}_{\alpha}$ remains optically thin in the largest part of the emitting wind-volume, such that porosity/vorosity effects can be neglected, contrasted to the analysis of (strong) UV resonance lines. Further details and consequences are discussed in Sect.6.3.

Thermal radio and FIR continuum emission. A conceptually different approach to "measure" mass-loss rates is to exploit the information contained in the (F)IR, (sub)millimeter and radio continua. Actually, this approach provides a remarkably reliable and easy-to-use tool, since it is based on rather simple processes.

The basic idea is to measure the excess relative to the flux predicted by photospheric models, which is emitted by free-free ("Bremsstrahlung") and bound-free processes in the wind. This excess flux becomes significant at longer wavelengths, due to the $\lambda^{2}$ dependence of the corresponding opacities and the corresponding increase of the effective IR/radio-photosphere, and can be translated into a mass-loss rate. In the following, we will concentrate on purely thermal emission, whereas non-thermal effects are discussed further below.

The approach to exploit the radio emission (here, the "excess" corresponds to the totally emitted flux, due to negligible photospheric emission) was independently developed by Wright and Barlow (1975 ${ }^{16}$ and Panagia and Felli (1975), and applied to larger O-star samples by Abbott et al (1980, 1981a) and Lamers and Leitherer (1993). A generalization towards shorter IR-wavelengths has been provided in a series of papers by Lamers and Waters (1984a,b) and Waters and Lamers (1984), who expressed the flux excess in terms of a curve of growth, from which the

\footnotetext{
16 these authors considered IR emission as well.
} 
velocity law and the mass-loss rate can be derived simultaneously. A first application of this method was the analysis of IRAS observations at 12,25 and $60 \mu \mathrm{m}$ of $\zeta$ Pup (Lamers et al 1984). Further references (regarding radio, submm and IR observations and analyses) can be found in Kudritzki and Puls (2000).

Following Lamers and Waters (1984a), the combined free-free and bound free opacity (in units of $\mathrm{cm}^{-1}$ ) at frequency $v$ can be written as

$$
\kappa_{v}=3.692 \cdot 10^{8}\left(1-\exp \left(-\frac{h v}{k T}\right)\right) \overline{z^{2}}(g(v, T)+b(v, T)) \frac{\gamma n_{i}^{2}}{T^{\frac{1}{2}} v^{3}},
$$

with electron temperature $\mathrm{T}, \overline{z^{2}}$ the mean value of the squared atomic charge, $g(v, T)$ and $b(v, T)$ the Gaunt factors for free-free and bound-free emission, $\gamma$ the ratio between electron and ion density, and $n_{i}$ the ion density, which relates to the mass density via $\rho=n_{i} \mu m_{\mathrm{H}}$ with atomic weight $\mu m_{\mathrm{H}}$. For long wavelengths and (almost) completely ionized $\mathrm{H}$ and $\mathrm{He}$, the major dependencies are

$$
\kappa_{v} \propto \frac{g(\lambda, T) \lambda^{2} \rho^{2}}{T^{3 / 2}}
$$

which increases strongly with $\lambda$ and $\rho$. Inserting typical values, it is easy to show that from the FIR on the continuum becomes optically thick already in the wind, and the emitting wind volume increases as a function of $\lambda$. E.g., the radio photosphere of typical O-supergiants is located at 100 stellar radii or even further out. Exploiting this knowledge, one can approximate $v(r) \approx v_{\infty}$ at radio wavelengths, and an analytical solution of the radiative transfer problem becomes possible for an isothermal wind:

$$
F_{v} \approx 23.2\left(\frac{\dot{M}}{v_{\infty}}\right)^{4 / 3} \frac{(v g(v, T))^{2 / 3}}{d^{2}}\left(\frac{\gamma \bar{z}^{2}}{\mu}\right)^{2 / 3}
$$

when $F_{v}$ is the observed radio flux measured in Jansky $\left(10^{-26} \mathrm{Wm}^{-2} \mathrm{~Hz}^{-1}\right), \dot{M}$ in units of $M_{\odot} \mathrm{yr}^{-1}, v_{\infty}$ in $\mathrm{km} \mathrm{s}^{-1}$, distance $d$ to the star in kpc and frequency $v$ in $\mathrm{Hz}$. Thus, the spectral index of thermal wind emission is 0.6 , since

$$
F_{v} \propto\left((v g(v, T))^{2 / 3} \propto v^{0.6} .\right.
$$

The scaling invariant of both radio and IR emission is $\dot{M} / R_{*}{ }^{1.5}$, rather similar to the quantity $Q$, if the object's angular diameter $\propto d / R_{*}$ remains fixed during the analysis. For typical O-stars located at $1 \mathrm{kpc}, F_{V}$ is of the order of $0.1 \mathrm{mJy}$ in the radio range $(2-20 \mathrm{~cm})$. Thus, OB-stars are generally weak radio sources (which prohibits the use of this method for extragalactic work!) and useful radio observations can be obtained only for nearby stars with rather dense winds. Moreover, since the spectral index of a non-thermal component (see below) is negative (e.g., $=-0.7$ for synchrotron emission), the radio domain can be more easily contaminated than shorter wavelengths such as the (sub)millimeter regime.

Again, due to the scaling with $\rho^{2}$, the major problem is the impact of clumping, already discussed by Abbott et al (1981a) and Lamers and Waters (1984b). Puls et al (2006) have used this additional dependence to derive the radial stratification of the clumping factor, see Sect.6.3. 
Non-thermal (NT) emission. So far, we have concentrated on the thermal emission. However, there is ample evidence of non-thermal emission in hot stellar winds. First observational findings have been reported by White and Becker (1983) and Abbott et al (1984), and Bieging et al (1989) concluded that roughly $30 \%$ of all massive stars were non-thermal emitters. To date, there are 16 O-type stars confirmed as non-thermal radio sources (van Loo et al 2006 and references therein). A recent review on non-thermal emission from massive stars (with emphasis on massive binaries) has been given by De Becker (2007), which includes the most up-to-date census of non-thermal radio emitters (the aforementioned $16 \mathrm{O}$-stars plus all known WR sources) including their multiplicity status.

Although it seems to be clear that the non-thermal emission is due to synchrotron radiation from relativistic electrons, different agents responsible for the acceleration are discussed in the literature: wind accretion onto compact objects (Abbott et al 1984), magnetic reconnection in single or colliding winds (Pollock 1989, see also Litvinenko 2003) and first-order Fermi acceleration (Fermi 1949), which, in the presence of strong hydrodynamic shocks, is also referred to as the Diffusive Shock Acceleration (DSA) mechanism (Blandford and Ostriker 1978, Bell 1978a, b). With respect to massive stars, the latter process has been invoked by White (1985, for important modifications to the original idea see Chen and White 1994) to explain the NT emission in suspected single stars, where the required shocks should be those embedded in the wind and created by the line-driven instability (Sect.6.2). Eichler and Usov (1993), on the other side, showed that also the shocks created by stellar wind collisions in early type binaries are potential sources of the observed NT emission, in line with even earlier suggestions by Williams et al (1987, 1990) regarding the non-thermal emission from the WC7+05 binary WR140. More recently, Pittard et al (2006) and Reimer et al (2006) have developed models applying the DSA mechanism to the corresponding physical conditions of hydrodynmical shocks in colliding wind binaries.

The presently favorized hypothesis is that non-thermal emission from massive stars is generally procuded by the latter mechanism. Two elements have simultaneously led to this idea. On the observational side, a number of systematic observational studies of NT emitters to investigate their multiplicity have revealed that the vast majority of them are confirmed (or at least suspected) binaries (see De Becker 2007). Note, e.g., that already Dougherty and Williams (2000) found strong indications that at least for WR-stars a massive companion is a prerequisite for the appearance of NT emission. On the theoretical side, van Loo et al (2006, based on Van Loo 2005) showed that the observed spectral shape of non-thermal emission cannot be reproduced by current (1-D) hydro-simulations applying the wind-instability scenario in presumed single $\mathrm{O}$-stars. They suspected that also all O-stars with non-thermal radio emission should be members of binary or multiple systems, where the non-thermal component is created in the shocks associated with colliding stellar winds.

In the present context, NT emission is particularly important because of its impact on the radio spectrum and flux. If present, it can strongly perturb mass-loss rate determinations based on the thermal radio flux, as, e.g., pointed out by Stevens (1995). In this context and assuming the above hypothesis of NT emission created in colliding winds, high angular resolution observations allowing for a direct disentangling of purely thermal (single star winds) and non-thermal (colliding- 
winds) emission in wide binaries will prove as highly valuable. However, let us also note that an additional thermal radio contribution may be produced by the shocked gas in the colliding-wind region itself (Stevens 1995, Pittard et al 2006), therefore contributing to some additional confusion.

\subsubsection{Methods based on NLTE atmospheres}

As outlined above, more recent wind diagnostics is mostly based on NLTE model atmospheres. At least three different methods can be envisaged:

(i) Synthetic spectra from self-consistent atmospheric models (comprising a NLTE + hydro-description, where the wind-structure relies on a line force resulting from self-consistent NLTE occupation numbers and the corresponding radiation field, see, e.g., Fig. 1 in Pauldrach et al 1994) are fitted to observations which cover a significant spectral range and are sensitive to both photospheric and wind conditions. The only input (= fit) parameters are $T_{\mathrm{eff}}, \log g, R_{*}$ (or different combinations, e.g., $T_{\text {eff }}, L_{*}, M_{*}$ ), the individual elemental abundances, and, if necessary, (stratified) clumping factors and a description of the X-ray/EUV emission. Besides the fact that such an approach is very time-consuming, it relies on the assumption that the complete underlying wind-physics is correctly treated. This method does not allow for an independent test of the theory itself. All inaccuracies/problems of the theory will, of course, show up in deviations between synthetic spectra and observations. Unfortunately, however, these will be interpreted in terms of peculiar abundances, clumping factors, radii etc., since for a given set of stellar parameters only one specific wind-structure (i.e., a specific combination of $\dot{M}, v_{\infty}$ and velocity field) is possible, which may not be correct. The better the theory, the better the results from such an approach, of course. An interesting application of method (i) will be presented in the context of determining stellar parameters of Central Stars of Planetary Nebulae via wind diagnostics.

(ii) Synthetic spectra from consistent atmospheric models (NLTE + hydro-description) are fitted to observations. In contrast to method (i), $\dot{M}$ and $v_{\infty}$ are adapted to fit the wind-lines, but not directly (as in method (iii) discussed below), but by varying the force-multiplier parameters $\mathrm{k}, \alpha, \delta$ (or equivalent quantities) in a reasonable ${ }^{17}$ way. The fit parameters are as above, augmented by the force-multiplier parameters. The advantage of this method relies in the possibility to obtain a physically justified stratification of the velocity field (and the indirect possibility to check the wind-theory, by checking whether the observations allow for reasonable force-multiplier parameters), but this advantage can also become a disadvantage when the actual velocity field cannot be matched by the hydrodynamic approach, due to shortcomings in the description. Method (ii) is usually applied in investigations using the atmosphere/wind-code WM-Basic (see Sect. 4.2.1), and impressive fits of the complete UV region have been obtained for a (small) number of stars, e.g., $\zeta$ Pup, $\alpha$ Cam (O9.5Ia) (Pauldrach et al 2001) and HD 93129A (O2If*, Taresch et al 1997), in the latter case including the optical region. Similar analyses of a larger sample of Galactic $\mathrm{O}$ supergiants and dwarfs have been performed by Bianchi and Garcia (2002) and Garcia and Bianchi (2004) (see below). Note that in almost all these cases the inclusion of the observed X-ray/EUV

\footnotetext{
17 within the range predicted by line-statistics, see pages $11 \mathrm{ff}$.
} 
emission (interpreted as due to the emission from cooling zones of shocks embedded in the wind, see Sect. 6.1) has been crucial to explain the ionization of highly ionized species such as O VI and S VI, see Pauldrach et al (1994, 2001) and Macfarlane et al (1993). These ions which show strong P Cygni lines in the FUV (e.g., Fig. 8) cannot be created in "standard" NLTE calculations based on a wind with an electron temperature of the order of $T_{\text {eff }}$, a problem realized already at the beginning of hot star UV spectroscopy and denoted as superionization (e.g., Lamers and Morton 1976, Castor 1979, Hamann 1980).

(iii) The last method enumerated here is the most frequently used. Synthetic spectra from a NLTE model are fitted to the observations, where the wind-structure is analytically described via a $\beta$ velocity law (Eq. 38), and a smooth transition between the analytical wind structure and a quasi-hydrostatic photosphere is adopted. Input-parameters (in addition to those from method (i)) are $\dot{M}, v_{\infty}$ and $\beta$. Such models (and those calculated by method (ii)) are called Unified Model Atmospheres (Gabler et al 1989), and are standard nowadays. Corresponding codes will be discussed in the next section.

Let us finally note that not only different methods can be (and are!) combined with (different) atmospheric codes, but that also the fit-optimization method can be different. Even in recent investigations, a "fit-by-eye" method has been frequently used, but more objective and automatic methods such as the optimization by genetic algorithms (Mokiem et al 2005) or using predefined grids in combination with a $\chi^{2} /$ maximum likelihood optimization (Lefever 2007) or via a principal component analysis (Urbaneja et al 2008) have also entered the spectral analysis of hot stars with winds.

NIR spectroscopy. The dramatic progress of IR astronomy in the last two decades has opened a completely new window for the systematic investigation of hot star winds, particularly for the analysis of dust-enshrouded, very young objects and samples in highly reddened clusters. Note also that the current generation of $10 \mathrm{~m}$ class ground-based telescopes has been optimized to observe in this wavelength range.

Also the IR spectra from hot stars show pure emission lines, wind-contaminated absorption lines and sometimes even P Cygni profiles, and these lines have to be analyzed by means of NLTE models due to strong NLTE effects in certain transitions. Since the IR continuum of objects with strong winds is formed already in the wind, however, IR lines may sample different depths inside the wind and provide additional information about the shape of the velocity field and particularly on the clumping properties.

The winds of hot stars of extreme luminosity and with strong IR emission characteristics in the Galactic Center have been investigated by different groups (e.g., Naiarro et al 1997b, Najarro 2006, Martins et al 2007), and Lenorzer et al (2004), Repolust et al (2005) and Geballe et al (2006) have demonstrated in how far stellar and wind parameters of "normal" OB-stars may be constrained by IRspectroscopy alone. One of the most important applications of IR line diagnostics, however, will be the measurement of mass-loss rates from stars with very weak winds by means of $\mathrm{Br}_{\alpha}$, as detailed in Sect. 5. 


\subsection{Recent results}

\subsubsection{OBA-stars}

Most of the relevant results in the field of quantitative spectroscopy of massive hot stars that have emerged with the new millennium are the outcome of more than ten years of considerable efforts made by different research groups to incorporate, in a realistic way, the effects of line-blanketing in NLTE-modeling of the atmospheres of massive stars. Indeed, the possibility to include the role of several thousands (up to millions) of metal lines was intimately coupled to the revolution of computer facilities. First approaches were made by Abbott and Hummer (1985) who modeled the effect of "wind blanketing" by introducing a wavelength dependent albedo representing the radiation reflected back onto the photosphere by electron and line scattering from the wind. Abbott and Hummer showed that the main result of "wind blanketing" was to heat the photosphere from the surface to the depth of continuum formation ("back-warming"), so that the resulting spectrum would resemble that of a hotter star. Thus, depending on the strength of the stellar wind $(\dot{M})$, the albedo could lead to a net reduction of the derived stellar $T_{\text {eff }}$ by up to $20 \%$. The main caveat of this approach was the neglect of photospheric "line blanketing", so that a unified picture could not be provided. As well, effort has been undertaken to incorporate the effects of photospheric "flux blocking" alone (Herrero et al 2000, Villamariz 2001). However, since only blocking was considered but no back-warming, revised $T_{\text {eff }}$ values were significantly higher than models without blocking.

One of the major steps in the blanketing affair was presented by Anderson (1985, 1989) who introduced the so-called "superlevels". Instead of dealing with all individual energy levels of metal species, several states with close enough energies were packed into one superlevel. Thus, the number of statistical equilibrium equations could be drastically reduced saving an enormous amount of computing time (but see, e.g., Lucy|2001 on potential problems inherent to this approach). After some pioneering work (Hauschildt 1992), by the end of the nineties, four major codes, PHOENIX (Hauschildt|1992), CMFGEN (Hillier and Miller 1998), WMBasic (Pauldrach et al 1998, 2001) and TLUSTY (Hubeny 1998, plane-parallel, no wind) could address line blanketing in a realistic way. Just a couple of years later two more codes, PoWR (Gräfener et al 2002) and FASTWIND (Santolaya-Rey et al 1997, Puls et al 2005) became available. For a brief comparison of the features of these codes, see Puls (2008).

Effects of line blanketing. To illustrate the two main direct effects of line-blanketing, we have made use of CMFGEN and computed a model for a late Bhypergiant with a strong wind. Fig. 9 shows the comparison of the blanketed/nonblanketed cases for the emergent flux distribution and temperature structure of the atmosphere. The models confirm the effects anticipated from early on and discussed above. We clearly see how the blocked flux in the UV emerges at optical and longer wavelengths. As the flux is blocked and a certain amount of photons are backscattered, flux (luminosity) conservation demands a more efficient photon diffusion in the inner photosphere. Since this effect is controlled by the temperature gradient, the temperature is increased in this region (back-warming) 

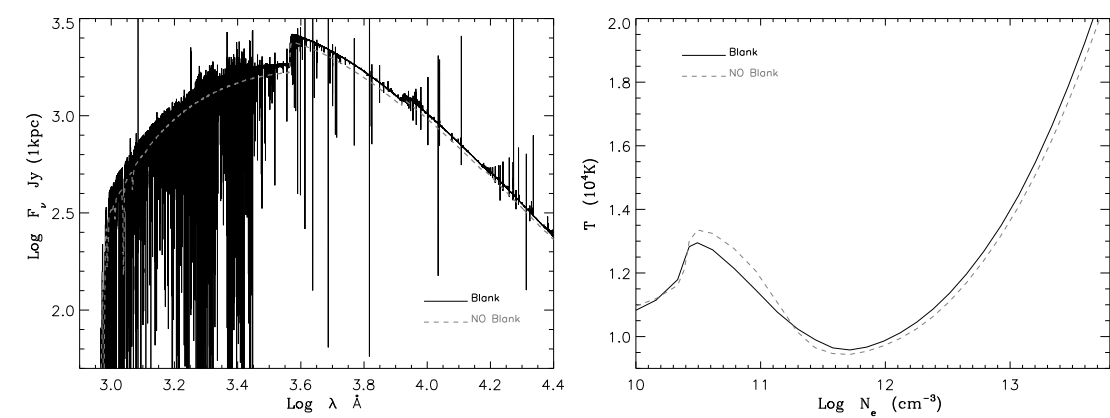

Fig. 9 Effects of line blanketing (solid) vs. unblanketed models (dashed) on the flux distribution ( $\log F_{V}$ (Jansky) vs. $\log \lambda(\AA)$, left panel) and temperature structure $\left(T\left(10^{4} \mathrm{~K}\right)\right.$ vs. $\log n_{\mathrm{e}}$, right panel) in the atmosphere of a late B-hypergiant. Blanketing blocks flux in the UV, redistributes it towards longer wavelengths and causes back-warming.
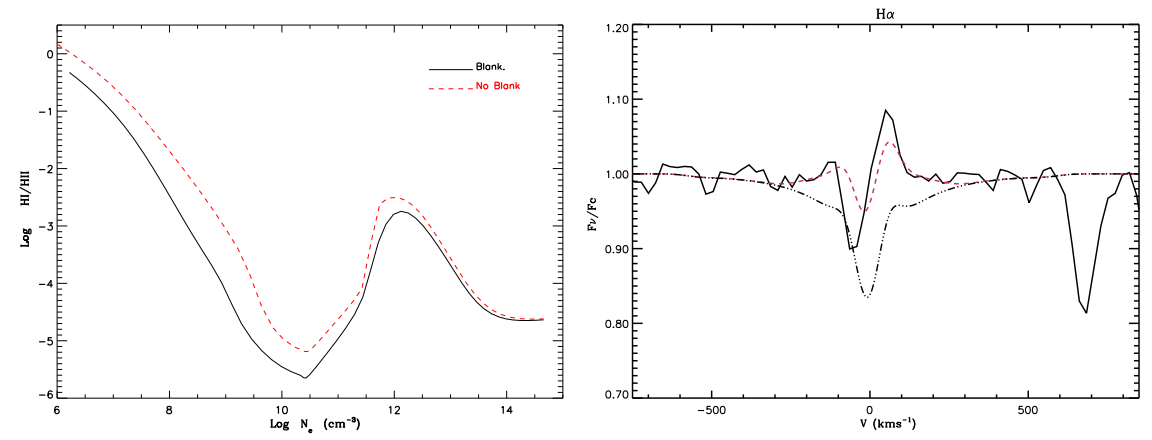

Fig. 10 Effects of line blanketing on the hydrogen ionization structure (log H I/H II vs. $\log n_{\mathrm{e}}$, left panel) and line profiles $\left(\mathrm{H}_{\alpha}\right.$, right panel) of a mid B-supergiant. The enhanced ionization caused by blanketing (solid vs. dashed) weakens considerably the $\mathrm{H}_{\alpha}$-profile (dashed-dotted) with respect to the unblanketed model (dashed) and requires an increase of $\dot{M}$ by up to a factor of three in B-supergiants to balance the effects of blanketing and to fit the observations (solid).

as shown in Fig. 9 Back-warming, in combination with the enhanced radiation field due to backscattering, will lead to enhanced ionization in the inner parts of the atmosphere. The question arising now is how the spectral lines will be affected and whether the effect of blanketing could be considered as a general shift in the ionizing conditions throughout the whole atmosphere (as found by Abbott and Hummer 1985) or whether it will affect differently the inner photosphere and the outer wind. Thus, we want to know whether this enhanced ionization will be kept throughout the atmosphere or whether the stellar wind will block enough ionizing radiation and decrease the ionization degree in the outer wind parts. The outcome of this competition will depend on the parameter domain in which the star is located, and will be very sensitive to stellar properties such as effective temperature, gravity and wind strength. For an illustration, we will compare the situation for a mid B-supergiant and a very hot early O-supergiant.

Fig. 10 shows the effects of line blanketing on the hydrogen ionization structure of the mid B-supergiant. Despite the strong wind, the enhanced ionization 

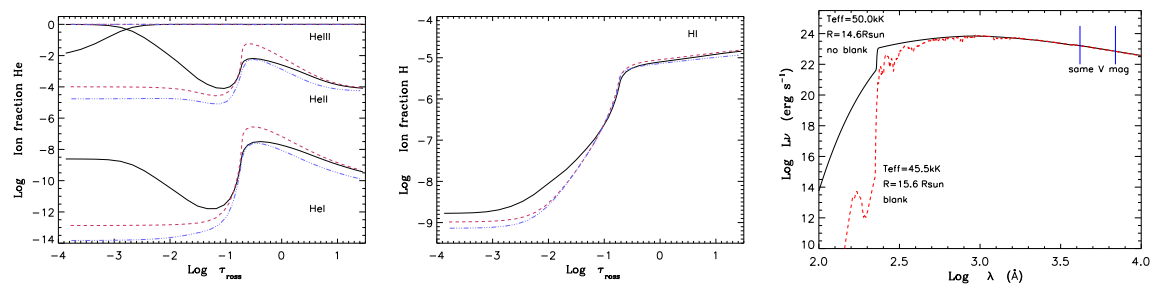

Fig. 11 Effects of line blanketing on the helium (left) and hydrogen (middle) ionization structure of an O3 If supergiant. Solid: $T_{\text {eff }}=45.5 \mathrm{kK}$, blanketed; dashed: $T_{\text {eff }}=45.5 \mathrm{kK}$, not blanketed; dashed-dotted: $T_{\text {eff }}=50 \mathrm{KK}$, not blanketed. See text. Right: Flux distribution of models with (dashed) and without (solid) blanketing matching the stellar line spectra and photometry.

caused in the inner photosphere is held throughout the wind. Further, the increased depletion of $\mathrm{HI}$ in the atmosphere through blanketing requires an enhancement of $\dot{M}$ by up to a factor of three to compensate the extra ionization and match the observed profile (see Fig. 10). Thus, for mid B-supergiants, blanketing affects majorly the derived mass-loss rate, while milder changes appear on the derived effective temperature. Similar trends have been found by Urbaneia (2004), Crowther et al (2006) and Markova and Puls (2008).

Fig. 11 displays the effects of line blanketing on the $\mathrm{He}$ and $\mathrm{H}$ ionization structure of an $\mathrm{O} 3$ If star. For the case presented in Fig. 11, we have selected three different models. The first one corresponds to a blanketed model with $T_{\text {eff }}$ $=45.5 \mathrm{kK}, \log g=3.7$ and $R=15.6 R_{\odot}$. The second model has the same parameters but no blanketing, while the third one, again not blanketed, is hotter, $T_{\text {eff }}=50 \mathrm{kK}\left(\log g=3.8\right.$ and $\left.R=14.6 R_{\odot}\right)$, and reproduces equally well the observed spectra of the $\mathrm{O}$ supergiant and the observed $\mathrm{V}$ magnitude. All models have the same wind-strength parameter $Q$. From Fig. 11-left we see that blanketing (solid) severely enhances the He ionization in the line forming region with respect to the cool unblanketed model (dashed), so that a degree of He ionization similar to the hot unblanketed model (dashed-dotted) is obtained by decreasing $T_{\text {eff }}$ by almost $5000 \mathrm{~K}$. In other words, the basic diagnostic for temperature determinations in $\mathrm{O}$ stars, namely the He ionization balance, reacts strongly to blanketing and causes the observed reduction on the effective temperature scale (for further details, see Repolust et al 2004).

Inspection of the impact of blanketing on the $\mathrm{H}$ ionization structure of $\mathrm{O}$ stars (Fig. 11-middle) tells us that only minor changes are produced in the ionization degree of $\mathrm{H}$ and, therefore, only small variations should be expected in the inferred mass loss rates (assuming they are derived from $\mathrm{H}_{\alpha}$, as $\mathrm{He}$ II 4686 would be severely altered). Finally, it should be noted that the relatively strong wind of the star blocks the He II ionizing radiation and forces recombination of He III to He II, with important implications on the number of He II ionizing photons (see also Fig 11-right). Thus, this is one case where the strong wind controls the ionization and outweighs the enhancement produced through backwarming in the inner regions.

First impact: The new temperature scale for massive OB stars. Vacca et al (1996) presented a compilation of the spectroscopic determinations of effective temper- 

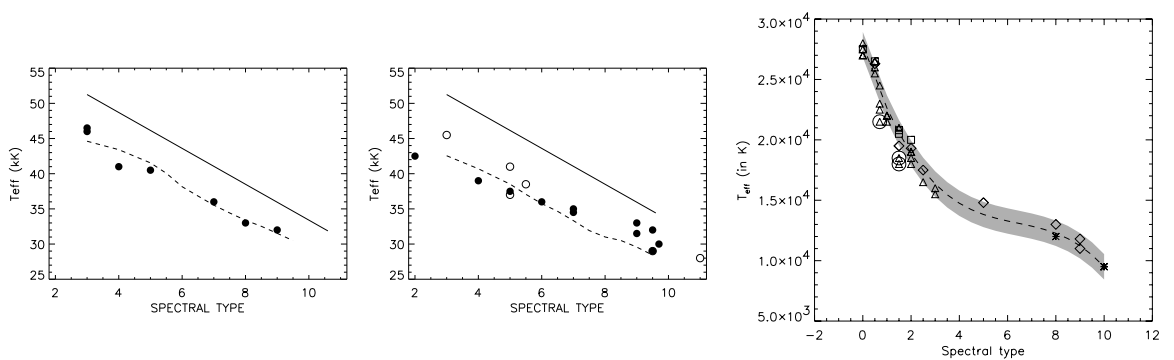

Fig. 12 The temperature scale for Galactic O-dwarfs (left) and supergiants (middle). The solid lines are for the Vacca et al (1996) scale, the dashed line for the "theoretical" scale defined by Martins et al (2005a), filled symbols are data from Repolust et al (2004) and open symbols are data from Herrero et al (2002). Right: The new temperature scale for B-supergiants from Markova and Puls (2008), combining results from Urbaneja (2004), Crowther et al (2006), Przybilla et al (2006), Lefever et al (2007) and their own data. See text.

atures of massive OB stars. They gave preference to the most recent calculations which at that time were mostly based on plane-parallel, hydrostatic, unblanketed NLTE model atmospheres. Interestingly, in their paper, Vacca et al (1996) warned about the significantly lower temperatures obtained by the few available windblanketed analyses when compared to unblanketed results.

Once the codes cited above became available, a series of papers were published mostly devoted to the issue of temperature scales. The first "modern" calculations pointing to a cooler temperature scale were those from Martins et al (2002) using CMFGEN. These authors limited their calculations to OB-dwarfs, so that the influence of mass-loss effects were negligible. Therefore, the main differences with Vacca et al were clearly due to line-blanketing. These differences could reach up to $4000 \mathrm{~K}$ for early types, and decreased towards $\mathrm{O} 9$ and $\mathrm{B} 0$ types, as can be appreciated in Fig. 112.

Herrero et al (2002) provided a temperature scale for supergiants in Cyg OB2 using FASTWIND. They found differences of up to $8000 \mathrm{~K}$. In this case both mass loss and line-blanketing played a role. These authors also showed that two stars with the same spectral type and luminosity class may have different effective temperatures if their wind densities are different. While the results from Herrero et al were based on the analyses of only seven Cyg OB2 supergiants, Repolust et al (2004) presented an analysis of 24 stars (17 supergiants and giants and 7 dwarfs), based on a slightly improved version of FASTWIND that confirmed the same trends.

(Almost) identical trends have been found by Martins et al (2005a) who provided 18 , by means of CMFGEN models, new temperature scales for massive OB stars of different luminosity classes. These scales agree quite well with those from Repolust et al, and confirm that new models result in effective temperatures that are several thousands Kelvin cooler for early and intermediate spectral types, decreasing towards late spectral types. A comparison of all these different temperature scales is presented in Fig. 12 .

Likewise detailed studies have been performed for B-supergiants (Urbaneja 2004, Crowther et al 2006, Lefever et al 2007, Markova and Puls 2008) which show

\footnotetext{
18 among other scaling relations for atmospheric parameters and ionizing photon numbers.
} 
that also the temperature scale of Galactic B-supergiants needs to be revised downwards. Compared to the unblanketed and "wind-free" scale by McErlean et al (1999), this amounts from 10 up to $20 \%$, the latter value being appropriate for stronger winds. The resulting new $T_{\text {eff-scale (described by a third order polynomial }}$ fit, with gray-shaded $1-\sigma$ uncertainties) is displayed in Fig. 12-right, and agrees perfectly with the results from an independent study by Trundle et al (2007).

Similar investigations have been performed for OB-stars in the Magellanic Clouds (MCs), in particular to study metallicity effects on the effective temperatures and mass-loss rates. Massey et al (2004, 2005) investigated a large sample of MC O-stars by means of FASTWIND, and provided a spectral-type- $T_{\text {eff }}$ calibration for the SMC. For the LMC, the situation remained unclear, since their sample was concentrated towards the hottest objects, O2-O4. Overall, it turned out that for a given spectral sub-type, $T_{\text {eff }}(\mathrm{SMC} \mathrm{dw})>T_{\mathrm{eff}}(\mathrm{MW} \mathrm{dw}) \approx T_{\mathrm{eff}}(\mathrm{SMC} \mathrm{sg})>$

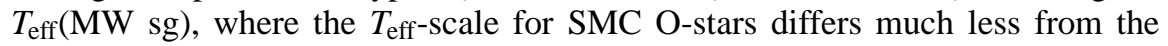
unblanketed Vacca et al (1996) calibration than the scale for their Galactic counterparts ('dw' = dwarfs, 'sg' = supergiants). This finding has been attributed to less blanketing and weaker winds because of the lower SMC metallicity (see below). For $d w a r f s$, most of the results derived by Massey et al. are in good agreement with investigations of different samples performed with CMFGEN (Martins et al 2004) and TLUSTY 19 (Bouret et al 2003), whereas a large number of MC supergiants analyzed by means of CMFGEN (Crowther et al 2002, Hillier et al 2003, Evans et al 2004a) turned out to be significantly cooler, even cooler than implied by the Galactic scale. In certain cases, this might be explained by wind-blanketing effects, since some of the discrepant objects are rather extreme, but other cases certainly need further inspection. Heap et al (2006) analyzed a sample of SMC Odwarfs and giants, and compared their results (using TLUSTY) with other studies. Though they stress the large scatter of $T_{\text {eff }}$ within individual sub-types, they found fair agreement with other investigations, except for Galactic data derived by Bianchi and Garcia (2002) and Garcia and Bianchi (2004) by means of a pure UV analysis (using WM-Basic), which seem to suggest systematically cooler temperatures than all other studies. Also this discrepancy has to be investigated in the near future.

The VLT-FLAMES survey of massive stars. One of the most important recent projects on OB-stars was the VLT-FLAMES survey of massive stars (FLAMES = Fibre Large Array Multi-Element Spectrograph). By means of this campaign, the massive stellar content of 8 young and old Galactic/MC clusters has been spectroscopically investigated, in order to answer urgent questions regarding (i) rotation and abundances (rotational mixing), (ii) stellar mass loss as a function of metallicity and (iii) fraction and impact of binarity. In total, $86 \mathrm{O}$-stars and $615 \mathrm{~B}$-stars have been observed at high resolution. An overview of objects and objectives has been given by Evans et al (2005, 2006), and a summary of important results can be found in Evans et al (2008).

Mokiem et al (2006, 2007a) 20 studied the O-/early B-star targets of the FLAMES survey in the SMC and LMC, respectively. They confirmed the basic results from Massey et al (2004, 2005), but refined the spectral-type- $T_{\text {eff }}$ scale, particularly

19 augmented by a CMFGEN analysis of the wind parameters.

20 by means of a genetic algorithm combined with FASTWIND, see Sect.4.1.2 
with respect to the LMC objects. They showed that, at least for O-dwarfs (which are not "contaminated" by additional wind-effects), the effective temperatures for a given spectral sub-type decrease with increasing metallicity, i.e., $T_{\text {eff }}(\mathrm{SMC})>$ $T_{\text {eff }}(\mathrm{LMC})>T_{\text {eff }}(\mathrm{MW})$. A similar result was derived by Trundle et al (2007) for the FLAMES B-type dwarfs, in this case based on TLUSTY model atmospheres.

Wind properties of $O B$ stars at different metallicities. Wind-properties of Galactic/MC OB-stars (primarily mass-loss rates, $\dot{M}$, and velocity field parameters, $\beta$ ) have been determined by numerous investigations (overlapping with those mentioned above and detailed in Puls 2008), from $\mathrm{H}_{\alpha}$ (partly combined with $\mathrm{He}$ II 4686) and the UV. In most cases, terminal velocities, $v_{\infty}$, have been adopted from UV-measurements (see Sect.4.1.1) and/or calibrations (Kudritzki and Puls 2000). Wind-momentum luminosity relations (WLR) have been inferred and compared with theoretical predictions, mostly from Vink et al (2000, 2001), see Sect. 3 . In summary, the following results have been obtained:

(i) For most $O$-learly $B$-stars, the theoretical WLR is met. Notable exceptions are O-supergiants with rather dense winds, where the "observed" wind-momenta appear as "too large" (see Markova et al 2004, Repolust et al 2004), which has been attributed to wind-clumping, see Sect. 6.3, and low luminosity O-dwarfs, where the "observed" wind-momenta are considerably lower than predicted, which has been denoted as the "weak wind problem" and will be covered in Sect.5.

(ii) The bi-stability jump in the B-star domain is represented by a gradual decrease in $v_{\infty}$ over the bi-stability region (Evans et al 2004b, Crowther et al 2006), by a factor of roughly 2.5 with respect to the ratio of $v_{\infty} / v_{\text {esc }}$. According to Markova and Puls (2008), the limits of this region are located at somewhat lower $T_{\text {eff }}$ (betweeen 18 to $23 \mathrm{kK}$ ) than those discussed by Vink et al (2000). Most important, however, is the finding that $\dot{M}$ changes over the bi-stability region by a factor (in between 0.4 and 2.5, Markova and Puls 2008) which is smaller than the predicted factor of 5. Thus it seems that the decrease in $v_{\infty}$ over the bi-stability region is not over-compensated by an increase of $M^{21}$. This finding seems to apply not only to the bi-stability region itself, but to the complete "low temperature region" (i.e., all mid/late B-type stars), where the predicted mass-loss rates are larger than those found from NLTE analyses based on unified atmospheres (Vink et al 2000, Trundle and Lennon 2005, Crowther et al 2006, Lefever et al 2007, Benaglia et al 2007, Markova and Puls 2008). This problem is reminiscent of the findings by Kudritzki et al (1999) who derived wind-momentum rates of mid B-supergiants which were much smaller (by a factor of 10) than those of early B-supergiants. Bearing in mind that Kudritzki et al (1999) used unblanketed atmospheres, even if applying an upwards "correction" of their mid B-supergiant mass-loss rates by a factor of three (accounting for the arguments from page 45), their results would still be in contrast to the predictions.

Nevertheless, a certain effect dividing hotter from cooler winds is present indeed, evident from the decrease in the ratio $v_{\infty} / v_{\mathrm{esc}}$ and likely related to the principal bi-stability mechanism. Moreover, as shown by Benaglia et al (2007, see also Markova and Puls 2008), a local increase of the ratio $\dot{M} / v_{\infty}$ seems to be present

21 at least not if the winds from hotter objects are not substantially stronger clumped than those from the cooler ones. 


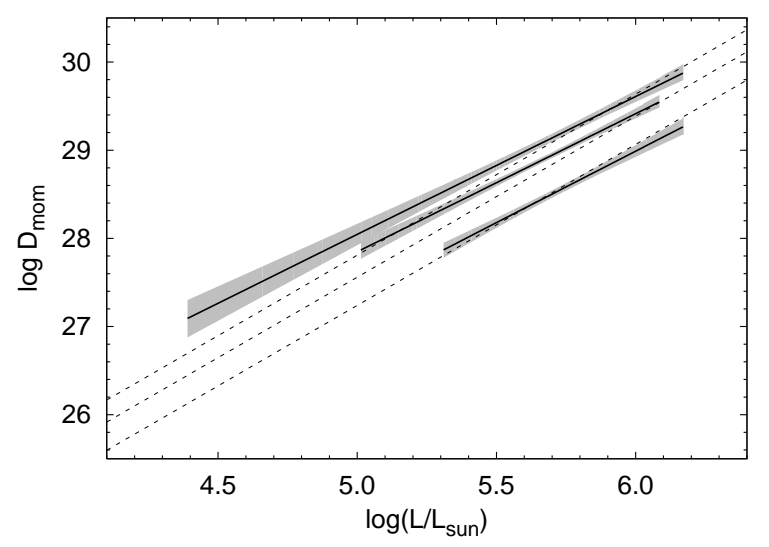

Fig. 13 Comparison of observed (solid, with grey shaded 1- $\sigma$ confidence intervals) and theoretically predicted (dashed, Vink et al 2000, 2001) WLRs for Galactic, LMC and SMC O-/early B-stars (from top to bottom). Adapted from Mokiem et al 2007b, see text.

at least inside the transition zone, which would partly support the predictions by Vink et al., though not regarding the mass-loss properties of objects below the jump. Further effort to clarify the situation is certainly needed.

(iii) Combining the results of the various investigations of Galactic OB-stars and A-supergiants (the latter from Kudritzki et al 1999), the corresponding WLRs (as a function of spectral type) extend over significant ranges in $T_{\text {eff }}$, assuming that clumping is not a strongly varying function of spectral type. Excluding weak winds, three rather well-defined relations for $\mathrm{O}$ and early $\mathrm{B}$-stars (roughly above $23 \mathrm{kK}$ ), for mid and late B-supergiants (between roughly $18 \mathrm{kK}$ and $10 \mathrm{kK}$ ) and for A-supergiants seem to exist, where the slope of the former two relations is rather similar, and the slope of the latter is steeper, in accordance with theoretical predictions. In particular, Kudritzki et al (1999) derived a slope corresponding to $\alpha^{\prime}=0.38 \pm 0.07$ for their sample of A-supergiants, which is in perfect agreement with corresponding results from line-statistics (page 13). Within the (re-defined) bi-stability region (18 to $23 \mathrm{kK}$ ), on the other hand, the scatter seems to be much larger, possibly due to ongoing changes in the global ionization equilibrium (Markova and Puls 2008).

Translated into the number of driving lines (or force-multiplier parameter $k$, cf. Eq. 15), the above results imply that this number remains rather constant over large ranges in $T_{\text {eff }}$ (again in accordance with theoretical predictions, Vink et al 2000 and Sect. 2.11, which are divided by the transition from Fe IV to Fe III and from Fe III to Fe II as the major driving agents. The "only" difference between these principal predictions and the observations is a quantitative one, regarding the location of the (first) bi-stability region and the degree to which extent $N_{\text {eff }}$ (or $k$ ) changes from one region to the other.

(iv) For a given luminosity, the mass-loss rates of SMC-stars are lower than for their Galactic counterparts, consistent with theory (Massey et al 2004, 2005). A more precise quantification of the metallicity dependence of the winds from $\mathrm{O}$ learly B-stars was possible within the FLAMES survey. From the analysis of the 


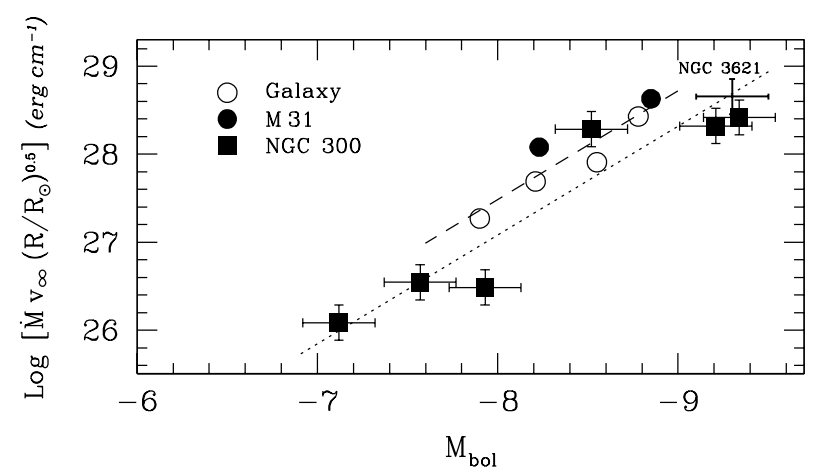

Fig. 14 WLR for Galactic and extragalactic A supergiants. Dashed: Linear regression for Galactic and M31 objects. Dotted: Galactic relation scaled to the mean abundance found for NGC 300 and NGC 3621, $Z / Z_{\odot}=0.4$. Adapted from Bresolin and Kudritzki 2004.

SMC/LMC objects by Mokiem et al (2006) and Mokiem et al (2007a), respectively, and in combination with data from previous investigations, Mokiem et al (2007b) derived the WLRs for Galactic, LMC and SMC objects, with rather narrow $1-\sigma$ confidence intervals, and showed that the wind-momenta strictly increase with metallicity $Z$ (i.e., the WLR of the LMC lies in between the corresponding relations for the MW and the SMC, see Fig. 13). Using $Z(\mathrm{LMC}) \approx 0.5 Z_{\odot}, Z$ (SMC) $\approx 0.2 Z_{\odot}$, and allowing for a modest "clumping correction" for dense winds (following the arguments by Repolust et al 2004, see also Markova et al 2004 and Sect. 6.3), they obtained $\dot{M} \propto Z^{0.72 \pm 0.15}$. This result is in very good agreement with theoretical predictions, both from (stationary) models (Sect. 3.4.1) and from line statistics (page 11). Mokiem et al (2007b) pointed out that the derived metallicity dependence should remain unaffected from potential future changes in the absolute values of mass-loss rates due to global clumping corrections, as long as the clumping properties were similar in winds of different metallicity.

Beyond the Magellanic Clouds. As already noted in our discussion of the (theoretical) concept of the WLR (Eq.16), one of the early aims to use this relationship was to derive extragalactic distances by purely spectroscopic means. Particularly advantageous for this purpose are early A-supergiants, because they are the visually brightest "normal" stellar objects. Also, problems of multiplicity and crowding are (almost) negligible because of their brightness, their short life-times, and their relatively old evolutionary ages (see Kudritzki et al 2008a). Moreover, their $\mathrm{H}_{\alpha}$-line can be used to measure $\dot{M}$ and $v_{\infty}$ simultaneously (e.g., Kudritzki et al 1999, Kudritzki and Puls 2000). Subsequently, a number of these objects have been analyzed in Local Group galaxies and beyond to compare with Galactic counterparts and to check the theoretical concept. A summary of the results has been given by Bresolin and Kudritzki (2004), highlighted in Fig. 14 which displays the corresponding WLRs. The relation for "solar" metallicity is defined by Galactic objects plus two M31 stars, and additional wind-momentum rates for 6 A-supergiants in NGC 300 and one object in NGC 3621 are displayed. Assuming that the latter stars have an abundance similar to the mean found for their host galaxies, $Z \approx 0.4 Z_{\odot}$, the dotted line provides the theoretical expectation when the 
Galactic WLR is scaled to the lower metallicity. It is evident that the WLR concept seems to hold. Details regarding extragalactic samples, observations and analyses can be found in Bresolin and Kudritzki (2004) and references therein.

After these promising investigations had been finished, however, no further attempt was made to derive distances via the WLR. The reason for that was that Kudritzki et al (2003) had developed an alternative possibility to use late B/early A-supergiants as standard candles, by means of what they called the Fluxweighted Gravity Luminosity Relationship. The authors showed that the fluxweighted gravity, $g / T_{\text {eff }}{ }^{4}$, and the absolute bolometric magnitude are strongly correlated for these objects, since they pass their short evolutionary phase with nearly constant luminosity and mass, and because luminosity and mass are related via $L \propto M^{x}$ with $x \approx 3$. Thus, the underlying difference in both methods is that the WLR method exploits the information on radius contained in the stellar wind, whereas the FGLR method relies on evolutionary facts.

As shown in the original paper and a follow-up study (Kudritzki et al 2008b), this new method to derive distances seems to have advantages over the WLR method, because of the high precision that is possible even for low-resolution observations ( $0.1 \mathrm{mag}$ in the distance module when analyzing 10 target stars) and its simplicity. E.g., the FGLR method requires much less (and only photospherid 22 ) parameters to be determined ( $T_{\text {eff }}, \log g$, and metallicity), and is also much less affected by additional dependencies. Remember, e.g., that the mass term in Eq.14 only partly vanishes for A-supergiants, since $\alpha^{\prime} \sim 0.4$ and not close to $2 / 3$ as in the OB-star case. Moreover, it is still unclear whether and in how far clumping affects the WLR method.

A first impressive demonstration of the power of the FGLR method has been given by Urbaneja et al (2008) who determined the distance to the Local Group Galaxy WLM with a distance modulus of $24.99 \pm 0.10 \mathrm{mag}$ (which is only $0.07 \mathrm{mag}$ larger than the most recent Cepheid distance). Further applications within the Araucaria project ("Measuring improved distances to nearby galaxies", Gieren et al 2005) are to be expected soon.

Central Stars of Planetary Nebulae. Although Central Stars of Planetary Nebulae (CSPN) are objects of significantly lower luminosity and of completely different evolutionary status, they have winds with a mean density similar to massive stars, and their wind-momenta seem to correspond to an extrapolation of the WLR of O-stars (Kudritzki et al 1997, 2006, Hultzsch et al 2007), though with a larger scatter. This finding must be regarded as an encouraging success of the interpretation of winds in terms of radiative driving and of the concept of the WLR. Let us point out that recent analyses by means of FASTWIND have suggested that some (but not all) of these winds are strongly clumped, which could be quantified because of the different reaction of the $\mathrm{He}$ II 4686 and the $\mathrm{H}_{\alpha}$ line on wind-clumping at temperatures around $T_{\text {eff }} \approx 30 \mathrm{kK}$ (Kudritzki et al 2006).

In an interesting study, Pauldrach et al (2004) analyzed the winds of 9 CSPN by means of WM-Basic and method (i) as outlined in Sect.4.1.2, i.e., they derived the complete set of stellar parameters by comparing self-consistent wind models with UV-observations. The information provided by the wind features $\left(v_{\infty}, \dot{M}\right)$

\footnotetext{
22 at least when discarding extreme objects with wind-affected SEDs.
} 
permits to derive the stellar radius via such models, since the corresponding dependencies $v_{\infty}=f\left(v_{\mathrm{esc}}\right)$ and $\dot{M}=f\left(L_{*}, M_{*}, \Gamma\right)$ allow for a unique solution for $R_{*}$ given the observational constraints (for a discussion of the underlying principles, see Kudritzki et al 1992). By this method, all quantities such as luminosity, mass, and distance can be constrained without relying on the (core)mass-luminosity relation taken from stellar structure theory and post-AGB evolution 23 Notably, the results by Pauldrach et al (2004) showed severe departures from this generally accepted relation, and masses from 0.4 to $1.4 M_{\odot}$ have been derived, with five out of the nine stars located close to (but not above) the Chandrasekhar mass limit for white dwarfs. Pauldrach (2005) argues that these objects might belong "to a not yet understood subgroup of CSPNs that evolve to white dwarfs which can end up as supernovae of type Ia" (see also Maoz 2008). For further progress on this issue, it needs to be clarified in how far the wind and spectrum synthesis calculations are not biased by contaminating effects. An important step towards this is the determination of precise distances to CSPN (to enable a measurement of the stellar radius) for which fairly resolved spectra can be obtained as well.

\subsubsection{Luminous Blue Variables}

What is an LBV? Luminous Blue Variables represent a short-lived $\left(\sim 10^{4}-10^{5}\right.$ years) phase of massive star evolution in which the stars are subject to significant effective temperature changes. They come in two flavors. The largest population of $\sim 30$ LBVs in the Galaxy and the Magellanic Clouds is that of the S Doradus variables with magnitude changes of 1-2 magnitudes on timescales of years to decades. These are the characteristic S Dor variations, which are represented by the dotted horizontal lines in Fig. 15. The general understanding is that the S Dor cycles occur at approximately constant bolometric luminosity (which has yet to be proven) - principally representing temperature variations. The second type of LBV instability involves objects that show truly giant eruptions with magnitude changes of order $3-5$ during which the bolometric luminosity most certainly increases. In the Milky Way it is only the cases of P Cygni and Eta Carina which have been witnessed to experience such extreme behaviour.

Whether these two types of variability occur in similar or distinct objects is not yet clear, however in view of the "unifying" properties of the LBV P Cygni it is highly probable that the S Dor variables and giant eruptors are related, that they are in a similar evolutionary state, and that they are subject to the same type of instabilities near the Eddington limit (see Vink 2009 for a review). We note that the true nature of the LBV instability has yet to be revealed.

LBV parameters and abundances. Reliable determinations of stellar properties in LBVs became possible only after the advent of unified model atmospheres. Even then, however, in some extreme objects like $\eta$ Car, the enormous complexity of the observed ground based spectra (Hillier and Allen 1992), contaminated by multiple emission regions, hinders a proper quantitative spectroscopic analysis. In this case, stellar parameters need to be determined either from "cleaner" wavelength regions (e.g., from the millimeter continuum, Cox et al 1995) or from "cleaner",

\footnotetext{
23 which is usually done when purely spectroscopic analyses such as outlined above are per-
} formed. 


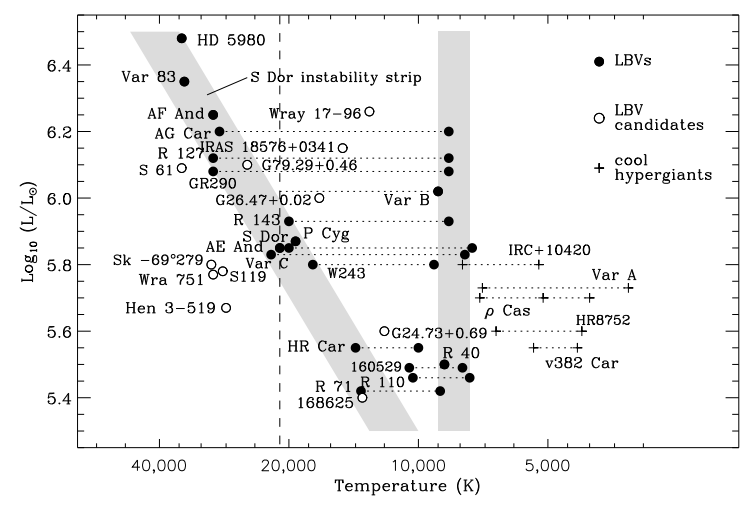

Fig. 15 The position of the LBVs in the upper Hertzsprung-Russell diagram. Note that the general population of blue supergiants that occupy the same part of the HRD are not plotted. The slanted band running from $30 \mathrm{kK}$ at high $L / L_{\odot}$ to $15 \mathrm{kK}$ at lower luminosity is often referred to as the S Dor instability strip. The vertical band at a temperature of $\sim 8000 \mathrm{~K}$ represents the position of the LBVs in outburst. The vertical line at $21000 \mathrm{~K}$ is the position of the observed bistability jump (the center of the observed bi-stability region). Adapted from Smith et al (2004).

resolved observations (e.g., using the HST, Hillier et al 2001, or the recent, really promising results from long baseline interferometers such as AMBER-VLTI, Weigelt et al 2007).

The outstanding wind density (with $\dot{M} \sim 10^{-3} M_{\odot} \mathrm{yr}^{-1}$ as estimated for this object, Cox et al 1995, Hillier et al 2001) places $R\left(\tau_{\text {Ross }}=2 / 3\right)$ at $80 \%$ of the terminal velocity, impeding any derivation of the hydrostatic radius. Nevertheless, $\mathrm{CNO}$ abundances could be determined and are consistent with those found for the surrounding nebula (for a recent review, see Najarro and Hillier 2008).

For less extreme LBVs such as P Cygni ( $\dot{M} \sim 10^{-5} M_{\odot} \mathrm{yr}^{-1}$, Najarro 2001), more detailed investigations could be carried out. A major breakthrough was obtained by Langer et al (1994) who presented a quantitative study of this object, combining evolutionary and hydrodynamical aspects. Subsequently, Najarro et al (1997a) were able to accurately constrain the main stellar properties, including Helium abundance, by means of homogeneous, unblanketed atmospheric models and UV to radio observations. These results were only minorly altered by utilizing blanketed models including clumping (Najarro 2001), which confirmed the presence of $\mathrm{CNO}$ processing and provided, for the first time, direct metallicity measurements for an LBV.

Apart from the problem of determining the (pseudo-hydrostatic) core radius (see also below), two other major difficulties arise when analyzing the spectra of LBVs. The first one is the determination of $T_{\text {eff. }}$. Only if both He I and He II are available (as for, e.g., He3-519 and AG Car, Smith et al 1994) a robust estimate may be obtained (although in some cases the He I spectra may be controlled by the presence of a binary companion, e.g., in $\eta$ Car). Otherwise, the effective temperature has to be obtained from simultaneous fits to lines from species with different ionization potentials (e.g. Si II, Mg II, Fe II). This is the case for the two LBVs in the Quintuplet cluster at the Galactic Center (the Pistol Star and \#362, Figer et al 


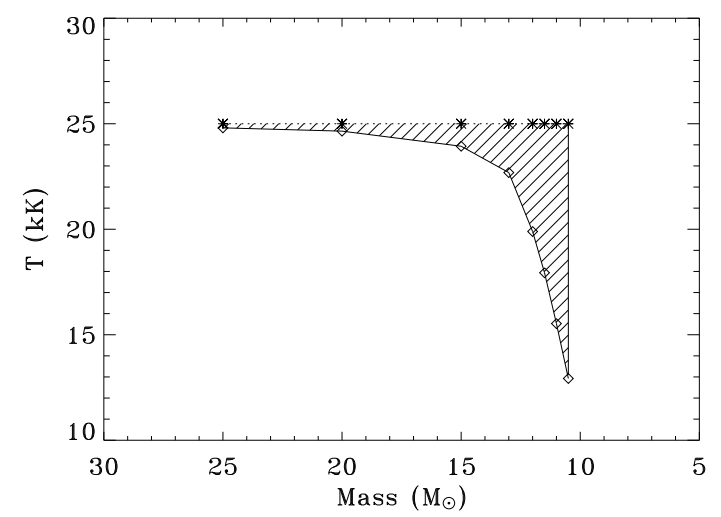

Fig. 16 The possible formation of a "pseudo-photosphere" for a low mass LBV. The difference in inner (dashed) and apparent temperature (representative for the size of the computed pseudophotosphere) is plotted against the stellar mass. These computations have been performed for a constant luminosity of $\log L / L_{\odot}=5.7$. The mass is gradually decreased until the object approaches the Eddington limit, and the apparent temperature drops as a result of low effective gravity and very high mass loss, forming a pseudo-photosphere. Adapted from Smith et al (2004).

1998, Geballe et al 2000, Najarro 2008). The second problem arises for low excitation LBVs and is related to the Helium abundance. Given the low excitation, $\mathrm{He}$ II recombines in the inner regions and the weak He I lines form very close to $R\left(\tau_{\text {Ross }}=2 / 3\right)$. In consequence, a degeneracy of the helium abundance appears (as for, e.g., HDE 316285, Hillier et al 1998) as basically for each $\mathrm{H} / \mathrm{He}$ ratio there is a $\dot{M}$-value that reproduces the observed $\mathrm{H}$ and $\mathrm{He}$ I lines equally well.

Do LBVs form pseudo-photospheres? It is evident that extreme objects such as $\eta$ Car with present-day mass-loss rates as high as $10^{-3} M_{\odot} \mathrm{yr}^{-1}$ form pseudophotospheres, but it is not generally accepted whether the mass-loss rates of the more common S Dor variables are large enough to form false photospheres. The issue is of particular interest for the following reason: whilst it appears that the photospheric temperatures of the objects in Fig. 15 change during HRD transits, there is the alternative possibility that the underlying object does not change its temperature but changes its mass-loss rate instead - resulting in the formation of an optically thick wind (e.g. Davidson 1987). As a result of increased mass loss it is hypothetically possible to form a pseudo-photosphere. Until the late 1980s this was the leading idea to explain the color changes of S Dor variables. Using more advanced NLTE model atmosphere codes, Leitherer et al (1989) and de Koter et al (1996) showed that the color changes consistent with measured LBV mass-loss rates are not large enough to make an LBV appear cooler than the temperature of its underlying surface. But is the issue of pseudo-photospheres in LBVs completely settled?

In NLTE codes used to investigate pseudo-photosphere formation in LBVs, the surface layers are based on the physical relation between $L, R$, and $T$, with 
$L=4 \pi R_{\mathrm{in}}^{2} \sigma_{\mathrm{B}} T_{\mathrm{in}}^{4}$. As the inner radius, $R_{\mathrm{in}}$, is chosen to lie in the photosphere, the input temperature $T_{\text {in }}$ does not necessarily equal the output apparent temperature, and one therefore defines the "effective" temperature $T_{\text {eff }}$ at a position where the thermalization optical depth equals $1 / \sqrt{3}$ in the optical $\mathrm{V}$ band (see de Koter et al 1996 and references therein). For the optically thin winds of OB stars, $T_{\text {eff }}$ equals $T_{\text {in }}$, but for some LBVs that lose mass at rates as high as $\dot{M} \sim 10^{-4} M_{\odot} \mathrm{yr}^{-1}$ cases might exist where there are significant differences between $T_{\text {in }}$ and $T_{\text {eff. }}$. If the wind is dense enough for the optical photons to emerge from a layer of rapid wind acceleration, the LBV can be considered to be forming a pseudo-photosphere. This may be favoured by lower LBV masses, as proximity to the Eddington limit provides larger mass loss and an increase of the photospheric scale-height.

Despite the proximity of LBVs to the Eddington limit, current consensus is that optically thick winds are generally not present in LBVs. However, Smith et al (2004) studied a special group of "missing" LBVs where the luminosity is relatively large (with $\log L / L_{\odot}=5.7$ ) but the stellar masses are possibly much lower than those of the classical LBVs (at $\log L / L_{\odot} \sim 6$ ). In other words, the lower-luminosity LBVs may well be in closer proximity to their Eddington limit. Figure 16 shows the potential formation of an optically thick wind for a relatively low-mass LBV. The size of the temperature difference (dashed vs. solid) is a proxy for the extent of the pseudo-photosphere. The figure demonstrates that for masses in the range 15-25 $M_{\odot}$, the winds remain optically thin, but when the stellar mass approaches $10 M_{\odot}$ and the star enters the regime near the Eddington limit, the photospheric scale-height blows up - resulting in the formation of a pseudo-photosphere. It should be noted that the reality of this scenario hinges critically on the input stellar mass, and LBV masses are poorly constrained from observations.

The line-driven winds during $S$ Doradus variations. Whilst most Galactic and Magellanic Cloud LBVs have been subject to photometric monitoring, only few have been analyzed spectroscopically in sufficient detail to understand the driving mechanism of their winds. As reported above, mass-loss rates are of the order of $10^{-3}-10^{-5} M_{\odot} \mathrm{yr}^{-1}$, whilst terminal wind velocities are in the range $\sim 100-500 \mathrm{~km} \mathrm{~s}^{-1}$. Of course, these values vary with $L$ and $M$, but there are also indications that the mass loss varies as a function of $T_{\text {eff }}$ when the $\mathrm{S}$ Dor variables transit the upper HRD on timescales of years. It is this aspect that provides us an ideal laboratory for testing the theory of radiation-driven winds.

The Galactic LBV AGCar is one of the more comprehensively monitored S Dor variables. Stahl et al (2001) investigated its mass-loss behaviour during the 1990s - modeling the $\mathrm{H}_{\alpha}$ line profile in detail. Vink and de Koter (2002) showed that the Stahl et al. mass-loss rates rise, drop, and rise, in line with radiation-driven wind models in which the variations in mass-loss rate and wind velocity are attributable to ionization shifts of $\mathrm{Fe}$ - the dominant element driving the wind.

It is relevant to mention here that this variable wind concept (basically wind bi-stability) has been put forward as a potential explanation for the circumstellar density variations inferred from quasi-periodic modulations in the radio lightcurves of some transitional supernovae (Kotak and Vink 2006). Additionally, the same mechanism is able to account for wind-velocity variations in the P Cygni absorption line spectra of supernovae such as SN2005gj (Trundle et al 2008). As 
current stellar models predict massive stars with $M \geq 30 M_{\odot}$ to explode at the end of the WR phase, rather than during or after the LBV phase, the ultimate implications could be far-reaching - impacting even our most basic understanding of massive-star evolution.

Continuum driven winds during giant eruptions. Whilst, during "quiet" phases, LBVs lose mass most likely via ordinary line-driving, they also appear to be subject to one or more phases of much stronger mass loss. For instance, the giant eruption of $\eta$ Car with a cumulative loss of $\sim 10 M_{\odot}$ between 1840 and 1860 (Smith et al 2003b) which resulted in the Homunculus nebula corresponds to $\dot{M} \approx 0.1-0.5 M_{\odot} \mathrm{yr}^{-1}$, which is a factor of 1000 larger than that expected from a line-driven wind at that luminosity. Such a strong mass loss has been frequently attributed to the star approaching or even exceeding the Eddington limi 24 , but linedriven mass loss is difficult to invoke, since the formal divergence of $\dot{M}$ for $\Gamma \rightarrow 1$ (Eq. 14) is accompanied by a vanishing terminal speed, $v_{\infty} \propto v_{\mathrm{esc}} \propto(1-\Gamma)^{0.5} \rightarrow 0$. (Remember that the wind-momentum rate is almost independent of $\Gamma$ ).

Building upon pioneering work by Shaviv (1998, 2001), Owocki et al (2004) investigated this problem in some detail and developed a simple theory of "porositymoderated" continuum driving in stars that exceed the Eddington limit. At first, they showed that the divergence of line-driven mass loss for $\Gamma \approx 1$ is actually limited by the photon tiring associated with the work needed to lift material out of the star's gravitational potential. The authors argue that continuum-driven winds in super-Eddington stars with a mass loss close to this tiring limit, $\dot{M}_{\mathrm{tir}}=L_{*}$ l $\left(G M_{*} / R_{*}\right)$, will result in a stagnating flow at a finite radius, which should lead to extensive variability and spatial structure. This should also be true for other instabilities (such as a convective one), which are expected to be present in the envelopes and atmospheres of stars close to or beyond the Eddington limit.

As first noted by Shaviv (1998), the porosity of such a structured medium can reduce the effective coupling between the matter and radiation by lowering the effective opacity in deeper layers (i.e., $\Gamma_{\mathrm{eff}}<1$ for $\Gamma>1$ ), thus enabling a quasihydrostatic photosphere, but allowing for a transition to a supersonic outflow when the structures become optically thin, i.e., $\Gamma_{\text {eff }} \rightarrow \Gamma$ for decreasing optical depth. As detailed in Sect.6.3.2, the effective opacity can be approximated by

$$
\kappa_{\mathrm{eff}} \approx \frac{\bar{\kappa}}{\tau_{\mathrm{c}}}=\frac{1}{h} \quad\left(\tau_{\mathrm{c}}>1\right), \quad \kappa_{\mathrm{eff}} \approx \bar{\kappa} \quad\left(\tau_{\mathrm{c}}<1\right), \quad \tau_{\mathrm{c}}=\int \kappa_{\mathrm{eff}} \mathrm{d} r
$$

for the optically thick and thin case, respectively, where $\bar{\kappa}$ is the mean opacity allowing for micro-clumping, $\tau_{\mathrm{c}}$ is the continuum optical depth and $h \equiv L^{3} / l^{2}$ the porosity length introduced by Owocki et al (2004) with $l$ the size and $L$ the separation of individual clumps. In other words, the porosity length is the photon's mean free path for a medium consisting of optically thick clumps.

Based on this porosity length and the Ansatz that it should scale with the gravitational scale height $H$ (in analogy to the mixing length theory), the authors derived scaling laws for a porosity-moderated, continuum-driven mass-loss rate from stars with $\Gamma>1$. For a super-Eddington model with a single porosity length $h \approx H$

\footnotetext{
24 here and in the following, we will consider a generalized Eddington limit, accounting for Thomson scattering and bf/ff continuum processes.
} 
and $\Gamma>>1, \dot{M} \approx L_{*} /(a c)$, consistent with the earlier findings by Shaviv (2001). This is much higher than typical for line-driven winds but still only a few percent of the tiring limit. Even higher $\dot{M}$ as implied from the ejecta of $\eta$ Car could be obtained by a power-law porosity model (in analogy to the line-strength distribution) which should mimic a broad range of clump scales. For power law exponents $\alpha_{p}<1, \dot{M}$ becomes enhanced over the single-scale model by a factor increasing with $\Gamma^{1 / \alpha_{p}-1}$, approaching the tiring limit under certain circumstances. Together with quite fast outflow speeds, $v_{\infty} \approx \mathscr{O}\left(v_{\text {esc }}\right)$ and a velocity law corresponding to $\beta=1$, the derived wind structure can actually explain the observational constraints of giant outbursts in $\eta$ Car and other LBVs. Moreover, the porosity model retains the essential scalings with gravity and radiative flux (the von Zeipel theorem, cf. Sect. 2.2.1 that would give a rapidly rotating, gravity-darkened star an enhanced polar mass loss and flow speed, similar to the bipolar Homunculus nebula. As stressed by van Marle et al (2008), continuum driving does not require the presence of metals in the stellar atmosphere. Thus, it is well-suited as a driving agent in the winds of low-metallicity and First Stars and may play a crucial role in their evolution.

\subsubsection{WR-stars}

The subject of WR stars was recently reviewed by Crowther (2007) to which we refer for details. Here we briefly discuss some of the recent progress in WR wind research.

WR stars can broadly be divided into nitrogen-rich WN stars and carbon (and oxygen) rich WC (and WO) stars. The principal difference between the two subtypes is believed to be that the $\mathrm{N}$-enrichment in $\mathrm{WN}$ stars is merely a by-product of H-burning, whilst the $\mathrm{C}$ in WC/WO stars is a sign of the fact that He-burning products have reached the stellar surface. As a result, WC stars are thought to be more evolved than WN stars.

We re-iterate that a classification as a WR star reflects purely spectroscopic terminology - signaling the presence of strong and broad emission lines in the spectra which are produced by strong winds. Such spectra can originate in evolved stars that have lost a significant amount of their initial mass, or alternatively from an object that has formed with a large initial stellar mass and luminosity. This latter group of WR stars may thus include objects still in their core H-burning phase of evolution.

During the last decade, we have witnessed at least two important developments in the study of WR stars. One advancement concerned the inclusion of line-blanketing in NLTE models which, contrary to the situation for the O stars (see Sect. 4.2.1), led to an increase in $T_{\text {eff }}$ and $L$ for WR stars (Schmutz 1997, Hillier and Miller 1999): The strong dependence of the ionization equilibrium of the WR wind on the interaction of the He II $\mathrm{L}_{\alpha}$ line with metal lines close in wavelength (Schmutz 1997) and the correct treatment of the He II $n \rightarrow 1$ series near the He II Lyman limit (Hillier and Miller 1998) lead to a lower ionization in the wind. Thus, a higher temperature is required to reproduce the observed WR lines.

A second development was the result of the realization that WR winds are clumped (e.g. Moffat et al. 1988) leading to a decrease in the empirical WR mass-

25 calculated here without the correction for $\Gamma$. 


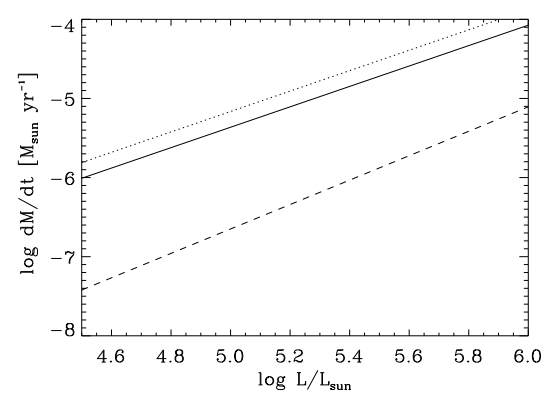

Fig. 17 Comparison of mass-loss rates from WR and Galactic OB-supergiants. Solid and dotted lines represent mean relations for $\mathrm{H}$-poor WN (solid) and WC stars (dotted) as provided by Nugis and Lamers (2000), with $\{Y=0.98, Z=0.02\}$ and $\{Y=0.55, Z=0.45\}$, respectively. See also Fig. 7 in Crowther (2007). The dashed line corresponds to Galactic O-supergiants, and has been taken from the WLR derived by Mokiem et al (2007b, see Fig 13), assuming prototypical values for $v_{\infty}\left(2000 \mathrm{~km} \mathrm{~s}^{-1}\right)$ and $R_{*}\left(15 R_{\odot}\right)$.

loss rate by a factor $2-4$ (e.g., Hamann and Koesterke 1998). Because on the one hand mass-loss rates have been down-revised, whilst on the other hand luminosities have been revised in an upwards direction, the burden for radiative acceleration to drive WR mass loss to values exceeding the single-scattering limit has become less severe. Nevertheless, compared to O-stars, WRs display significantly larger wind densities and mass-loss rates (factors $\gtrsim 10$ for the latter, see Fig. 177). In contrast, the terminal velocities of WR stars are comparable to those from Ostars, of the order of $1000 \mathrm{~km} \mathrm{~s}^{-1}$ (late WNs and WCs) to $2500 \mathrm{~km} \mathrm{~s}^{-1}$ and higher (early WNs and WCs), see Table 2 in Crowther (2007).

It is relevant to note that despite the fact that WR temperatures are now calculated to be larger than a decade ago thanks to the inclusion of metal-line blanketing, the inner core radii (which are a factor of several smaller than the "photospheric" radii) computed from the most sophisticated NLTE models are still a factor of several $(\sim 3)$ larger than those predicted for the He-main sequence by stellar evolution models. In other words, the problem of how WR winds are connected to their stellar core is still lingering, despite the impressive progress recently reported (Crowther 2007).

One might now question why there is a difference between the winds from WR and O-stars if also WR winds were accelerated by radiative line-driving, as supported by a number of investigations (for references, see Sect. 3.4.3). This problem has been extensively discussed in the literature, but cannot yet be conclusively answered. However, there are some facts and findings which might serve as a guideline.

(i) Since for a given luminosity the initiated mass-loss rate is considerably larger than in O-stars, whereas the terminal velocities are quite similar, the momentum transfer in WR winds must be more efficient. This efficiency is supposed to be related to very efficient multi-line scattering events, possible because of photon trapping (Lucy and Abbott 1993, Springmann and Puls 1998): In O-star winds the ionization equilibrium is almost frozen-in, leading to gaps in the frequency distribution of the lines and thus to a "premature" photon escape when such gaps 
are encountered. In WR stars with a high core temperature and a larger winddensity, the ionization equilibrium is stratified, and the previous gaps are closed due to the emergence of new lines from a new ionization stage. Thus, photons become trapped instead of escaping, and can transfer more momentum. (ii) Moreover, again due to the larger wind-densities, the ionization equilibrium in the outer WR winds is shifted towards lower stages than present in the corresponding Ostar winds, and the number of lines is larger, leading to an efficient acceleration of the outer wind. (iii) Following the ionization stratification inwards, there is a (hot) iron opacity peak around $160 \mathrm{kK}$ in the deeper atmospheres of WR star26 (Nugis and Lamers 2002) which according to Gräfener and Hamann (2005) provides the required opacity for initiating WR winds (as shown for a WC5-type object). (iv) Finally, it should be mentioned that theoretical models of WR stars display the strongest strange-mode instabilities (Gautschy and Glatzel 1990; for a review, see Glatzel and Chernigovski 2001) found so far (Glatzel et al 1993). Recent non-linear simulations (Wende et al 2008) have shown that Wolf-Rayet models can reach radial velocity amplitudes which amount to up to $30 \%$ of their escape velocity. The corresponding mechanical energy transferred by shock waves to the stellar atmosphere might easily help or even dominate the initiation of mass-loss and the acceleration of the lower WR wind. But note also that Moffat et al (2008) found no coherent oscillations in the hot WC-star WR 111 after photometrically monitoring this object with the microsatellite MOST for three weeks. The derived upper limit for coherent Fourier components turned out to be almost two orders of magnitude below corresponding predictions from strange-mode pulsation simulations.

Returning to the (more conventional) WR wind model by Gräfener and Hamann (2005), let us stress that there are two crucial aspects which had to be adopted in order to enable a consistent wind driving: a rather large clumping in the outer wind $\left(f_{\mathrm{cl}}=50\right.$, but see Sect. 6.3.2), and the proximity of WRs to the Eddingtonlimit. The latter was suggested by these authors as the most critical parameter for initiating a strong mass flux (see Eq. 14 and Sect. 3.3). Note that there are no present self-consistent models of WR winds with the underlying star being (far) away from this limit!

We now turn to the empirical dependence of WR winds on initial metallicity. Until a few years ago, there were both indications for as well as against the existence of a mass-loss- $Z$ dependence for WR winds, however quantitative numbers on the exponent $m$ of the mass-loss $Z$ exponent were not provided until theoretical results of a $\dot{M}-Z$ dependence for WR stars became available (Vink and de Koter 2005 and Sect. 3.4.3), and empirical exponents have been quoted for both WN $(m$ $\sim 0.8)$ and $\mathrm{WC}(m \sim 0.6)$ stars - similar to those predicted.

An oft-used formula in stellar evolution computation for WR winds is the one provided by Nugis and Lamers (2000, see also Fig. 17) in which the mass-loss rate depends on both the $\mathrm{He}(\mathrm{Y})$ and the total metallicity $(\mathrm{Z})$. We note that the $Z$ in this formula represents the total $Z$, whilst, theoretically, $\mathrm{Fe}$ is thought to be the dominant wind driving ion for WR stars (Vink and de Koter 2005, Gräfener and Hamann 2008). For this reason we should not attach too much physical significance to the $Z$ dependencies quoted by Nugis and Lamers (2000). Nevertheless, the formula may well provide a proper representation of empirical mass-loss rates (cf. Fig. 7

26 Additionally, there is also a weaker, (cool) opacity peak around $40 \mathrm{kK}$. 
in Crowther 2007), and since a general theoretical description of mass-loss rates from WR stars is no yet available, they probably present the most reliable formulae for use in stellar evolution calculations of He stars.

Particularly relevant for the issue of LGRBs are the rotational properties of their progenitor stars. Owing to the broad emission lines in their spectra, traditional $v \sin i$ studies are not feasible for WR stars. Luckily, there is a powerful alternative in the form of linear spectropolarimetry. Apart from its role in constraining wind clumping properties (Sect.6.3.2), large scale asymmetries resulting from rotationally-flattened winds may also be probed with the technique. Harries et al (1998) and Vink (2007) studied samples of resp. Galactic and LMC WR stars and found equal incidences of polarization "line effects" in WR stars at $Z_{\odot}$ and $\sim 0.4$ $Z_{\odot}$. As there exists no evidence for significant differences in rotational properties of the presumed progenitors of LGRBs, these results suggest that one needs to study lower than LMC $Z$ environments to detect a significant population of rapid rotators at the end of their lives, if there is a low $Z$ bias for the creation of LGRBs via the collapsar model.

\section{The weak wind problem}

First statistically relevant evidence for the presence of O-stars with theoretically unexpected thin, weak winds was provided already by Chlebowski and Garmany (1991). From studies of the wind and X-ray properties of O-stars, they found that the mass-loss rates derived for the $O$ - $d$ warfs of later spectral type were significantly lower (up to a factor of 10) than expected from the $\dot{M} \propto L^{x}$ power-law relation (Sects.2.1 and 3) known for brighter O-stars. In the same year, Kudritzki et al (1991) analyzed the UV spectrum of the B-type giant $\beta$ CMa (B1 II) and concluded that its theoretically predicted mass-loss rate $\left(3 \cdot 10^{-8} M_{\odot} \mathrm{yr}^{-1}\right)$ had to be reduced by a factor of 5 to reproduce the Si IV $\lambda 1397$, C III $\lambda 1175$, and C IV $\lambda 1550$ profiles. This result was confirmed by Drew et al (1994) from an analysis of the ROSAT X-ray emission of $\beta \mathrm{CMa}$, who claimed a similar reduction for the $\mathrm{B} 2 \mathrm{II}$ star $\varepsilon$ CMa and presented two alternative explanations for this behaviour. The first one invoked the X-ray emission from an "outer zone of hot shocked gas" to penetrate far enough inwards to increase the ionization around the critical point, thus reducing the radiative acceleration and the mass-loss rate. Alternatively, the source of wind heating could be due to ion-drag effect:27 (Springmann and Pauldrach 1992). Using corresponding scaling relations from Gayley and Owocki (1994), Drew et al argued that the winds of these two B-giants were not significantly affected, leaving wind-shocked $\mathrm{X}$-rays as the most plausible heat source.

Using $\mathrm{H}_{\alpha}$ as a more reliable mass-loss indicator, Puls et al (1996) showed that the observed WLR for O-type dwarfs exhibited a severe curvature towards very low wind-momenta for luminosities lower than $10^{5.3} L_{\odot}$, indicating a break-down of the WLR in that domain. It should be stressed, however, that their sample was biased towards higher luminosity stars for which $\mathrm{H}_{\alpha}$ could be optimally used. In fact, their $\mathrm{H}_{\alpha}$-diagnostics could provide only upper $\dot{M}$-limits for the lower lumi-

\footnotetext{
27 frictional heating accompanying the collisionial momentum transfer between radiatively accelerated metals and the bulk wind matter, see page 6
} 

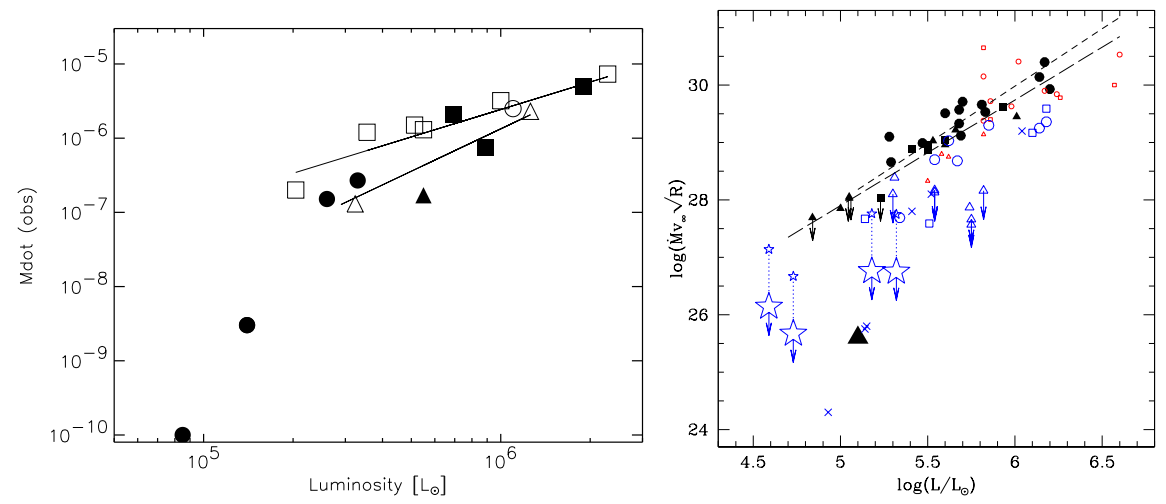

Fig. 18 The weak wind problem. Left: Mass-loss rates for O-dwarfs in NGC 346 (Bouret et al 2003, LMC, filled circles), compared to "normal" winds from Galactic (squares) and other SMC stars (triangles) as analyzed by Puls et al (1996). Right: Modified wind momenta as a function of stellar luminosity for O-stars (adapted from Martins et al 2004). Filled and open symbols correspond to Galactic and LMC (small red)/SMC (blue) stars, respectively. Luminosity class $\mathrm{V}$, III and I are represented by asterisks, squares and circles. Note the low wind-momenta of the SMC objects and the Galactic star 10 Lac (large triangle).

nosity objects, and subsequently the $\dot{M}$-determination in weak-winded stars was deferred until unified atmosphere models were able to do so.

From a theoretical point of view, physical possibilities to explain this behaviour continued to be explored (Puls et al 1998, Owocki and Puls 1999, Babel 1995, 1996, Krtička and Kubát 2000), where three effects were considered as particularly promising and have been reviewed by Kudritzki and Puls (2000). The first one relates to the potential decoupling of metal ions from the bulk plasma, which should happen below a critical density when the Coulomb-collisions become ineffective (Sect. 2.1 and references therein). The second one refers to the shadowing of the wind-driving lines by the photospheric ones, which considerably lowers the line force, resulting in a reduction of $\dot{M}$, especially in the case of B-dwarfs (Babel 1996). Finally, for low mass-loss rates where the continuum is thin throughout the transonic region, curvature terms of the velocity field leading to source function gradients may reduce the line-accelerations to values much smaller than in the standard computations, resulting again in reduced mass-loss rates (Puls et al 1998, Owocki and Puls 1999). Unfortunately, due to the lack of reliable $\dot{M}$ estimates it remained open to which extent the above processes could account for the weak wind problem.

First results with modern unified codes using both UV and optical lines were obtained by Herrero et al (2002). For the Galactic O9V star 10 Lac they derived an upper limit of $\dot{M} \leq 10^{-8} M_{\odot} \mathrm{yr}^{-1}$ from the optical and $\dot{M} \sim 10^{-10} M_{\odot} \mathrm{yr}^{-1}$ by including additional constraints from the UV, which is more than one dex below the theoretical predictions (see also Fig 19). Subsequently, Bouret et al (2003) and Martins et al (2004) obtained similar results for a larger sample of SMC O-dwarfs (see Fig. 18). The latter authors studied in detail the reliability of their mass-loss rate estimates derived from UV spectra and concluded that, at most, the true $\dot{M}$ could be underestimated by a factor of six. With these determined mass-loss rates 


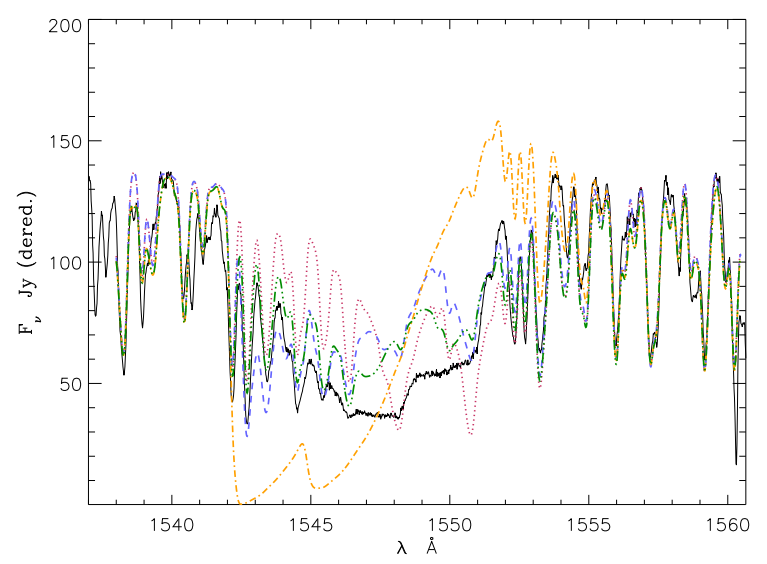

Fig. 19 The role of X-rays in the O-dwarf weak wind problem. Model fits to C IV $\lambda 1550$ from the $\mathrm{O} 9 \mathrm{~V}$ star $10 \mathrm{Lac}$, using different mass-loss rates. Without X-rays, an $\dot{M}$-value as low as 2.5·10 $10^{-10}$ (dashed blue, $\dot{M}$ in $M_{\odot} \mathrm{yr}^{-1}$ ) is required, whilst a value of $10^{-10}$ (dotted red) produces a much too weak profile, and a value of $10^{-9}$ (dashed-dotted orange) results in a too strong, P Cygni type profile. If X-rays are included $\left(L_{x} / L_{*} \sim 10^{-6.7}\right.$, dashed-dotted-dotted green curve), however, the observations can be reproduced with $\dot{M}=10^{-9} M_{\odot} \mathrm{yr}^{-1}$. Adapted from Najarro, Puls \& Herrero (in prep. for A\&A).

Martins et al revisited in detail the physical possibilities discussed above and concluded that none of them could account for such a strong mismatch between observed and theoretically expected $\dot{M}$. They speculated that although the physical mechanism leading to such weak winds remained unknown, the low mass-loss rates were intrinsically related to the youth of the stars, possibly testifying the phase of wind onset in young O-stars shortly after their formation.

Martins et al (2005b) extended this study to Galactic objects by performing a combined UV and optical analysis of a sample of $12 \mathrm{O}$-dwarfs. Again, a mismatch as high as a factor of 100 was found for later spectral types with $\log \left(L / L_{\odot}\right)<$ 5.2. A major step was the investigation of the effects of X-rays, magnetic fields, advection and adiabatic cooling in the determination of $\dot{M}$ from UV spectra. This lead Martins et al to conclude that the inclusion of X-ray emission (likely related to magnetic mechanisms, see Sects.2.2.2 and 6.5.1) in models with low density is crucial to derive accurate mass-loss rates from UV lines, while it was found to be unimportant for high density winds. This is shown in Fig 19, where models with and without X-rays and different mass-loss rates are compared for C IV $\lambda 1550$ from $10 \mathrm{Lac}$.

One important conclusion from the above studies is that the discrepancy in the derived mass-loss rates seems not to be related to metallicity, as it is present in both the Galactic and SMC stars. Interestingly, however, the break-down of the WLR starts at earlier spectral types (higher luminosity) for the lower metallicity environment of the SMC (around O6-6.5V, Bouret et al 2003, Martins et al 2004) than for the Galaxy (O9V, Martins et al 2005b). Thus, although the principal problem appears to be unrelated to metallicity, its onset (with respect to luminosity) could be linked to the metal content. 

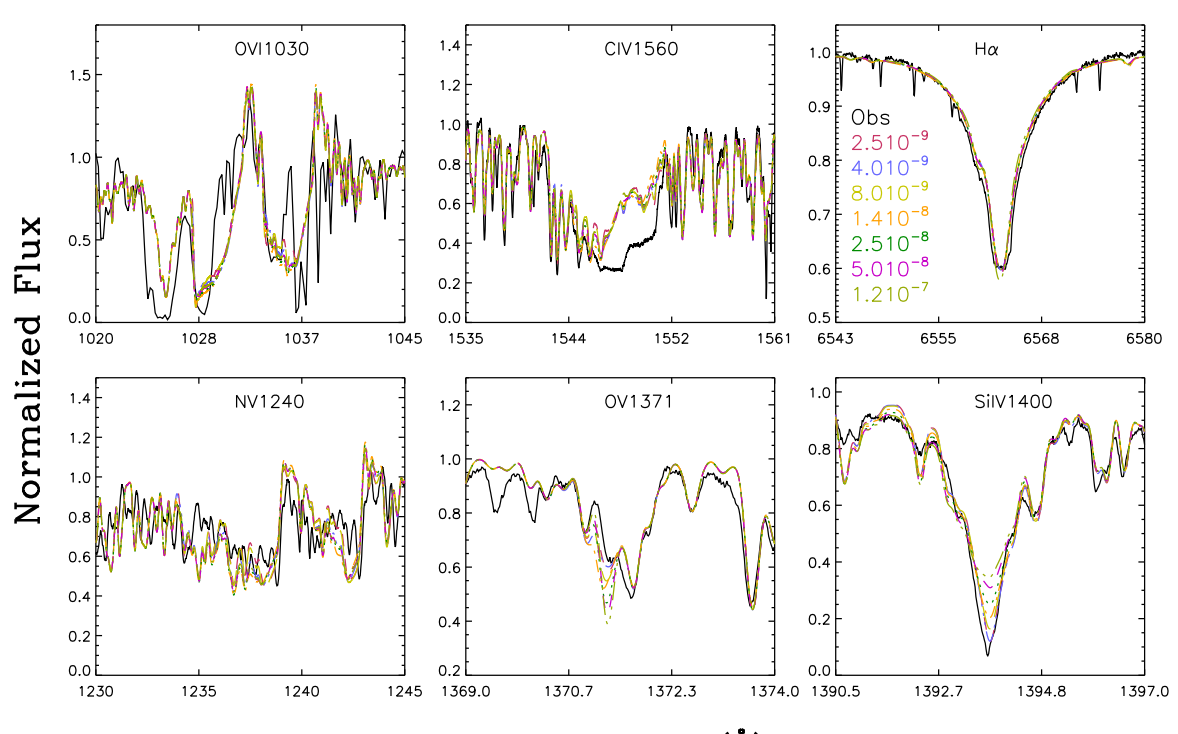

Wavelength $(\AA)$

Fig. 20 The $\dot{M}$-X-ray degeneracy. Model profiles computed for different mass-loss rates and scaled X-ray fluxes for strategic UV lines from $10 \mathrm{Lac}$, with $\dot{M}$-values in units of $M_{\odot} \mathrm{yr}^{-1}$. Adapted from Najarro, Puls \& Herrero (in prep. for A\&A).

Not only the mass-loss rates, but also the terminal velocities seem to behave unexpectedly in weak winds. The values derived by Martins et al (2005b) lie far below the expected and observed average value of $v_{\infty} / v_{\mathrm{esc}} \approx 3$ for stars above the bi-stability jump, rather close to a ratio of unity. Given the low wind densities, only upper limits could be obtained from C IV $\lambda 1550$, which constitutes the line with the highest sensitivity to $\dot{M}$ and $v_{\infty}$ for these late spectral types. Inverting the scaling relations for radiation driven winds (Eq. 13), Martins et al argued that a value of $\alpha \sim 0.3$ was required to reproduce the observed ratios, again quite different from the expected $\alpha \sim 0.6$ value. Interestingly, the mechanism related to X-ray emission proposed by Drew et al (1994, see above) was recalled to explain such a low value.

A crucial point regarding the reliability of UV $\dot{M}$-determinations for weakwinded stars is posed in Fig. 20. Here, we show the diagnostic UV and $\mathrm{H}_{\alpha}$ profiles for a set of models where the mass-loss rate is varied by almost two orders of magnitude whilst the X-ray luminosity is increased in parallel to keep the ionization structure and thus the UV lines at the observed level as far as possible. Note that the changes imposed on the wind do not reach the photospheric levels, which is obvious from the fact that the underlying UV iron forest does not change. Apart from the cores of Si IV $\lambda 1397$ and O v $\lambda 1371$, the observations can be equally well reproduced with any $\dot{M}$ combined with an appropriate $L_{x}$. Thus, no independent UV "measurement" of mass-loss rates may be obtained unless the $\mathrm{X}$-ray properties of the star are accurately known. 

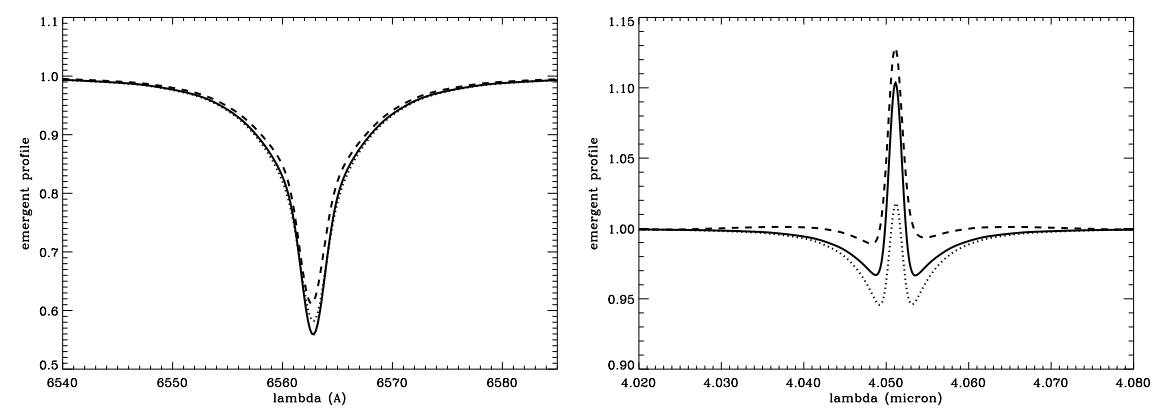

Fig. 21 Left: Synthetic $\mathrm{H}_{\alpha}$ profiles for $\tau$ Sco, at different mass-loss rates $\dot{M}=2 \cdot 10^{-9}, 2 \cdot 10^{-8}$ and $6 \cdot 10^{-8} M_{\odot} \mathrm{yr}^{-1}$ (dotted, bold, and dashed, respectively). Note the insensitivity of $\mathrm{H}_{\alpha}$ at lowest mass-loss rates. Right: Synthetic $\mathrm{Br}_{\alpha}$ profiles, for the same models. The line is a sensitive diagnostics for $\dot{M}$ even at lowest values.

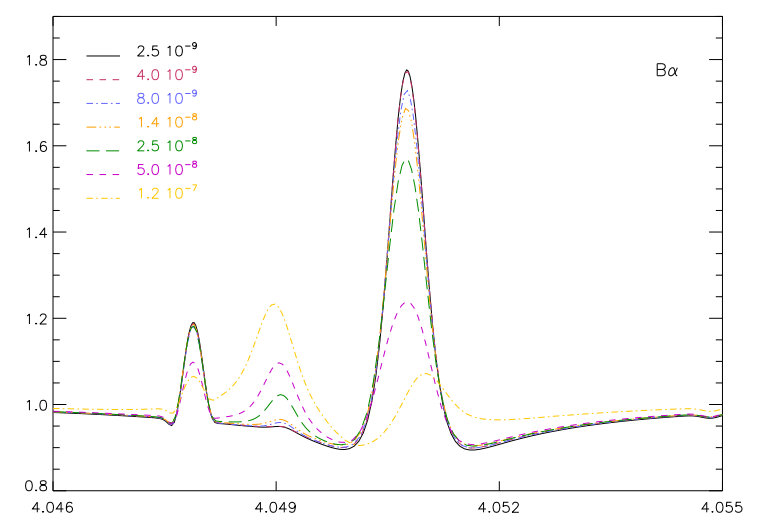

Fig. 22 Synthetic $\mathrm{Br}_{\alpha}$ profiles for $10 \mathrm{Lac}$ for the model sequence displayed in Figure20 Note the sensitivity of $\mathrm{Br}_{\alpha}$ on $\dot{M}$ even at lowest values. At larger $\dot{M}$, the He II-component becomes sensitive on the amount of X-ray emission as well (see text).

Consequently, a reliable $\dot{M}$-diagnostics is required that does not suffer from effects such as X-rays, advection, or adiabatic cooling. This is provided by the $\mathrm{Br}_{\alpha}$ line in the infrared. The relevance of $\mathrm{Br}_{\alpha}$ has been pointed out already by Auer and Mihalas (1969), who predicted that even for hydrostatic atmospheres the (narrow) Doppler-cores should be in emission, superimposed on rather shallow Stark-wings. The origin of this emission is a combination of various effects in the upper photosphere and the transonic region that depopulate the lower level of $\mathrm{Br}_{\alpha}, n=4$, stronger than the upper one, $n=5$. As detailed by Najarro, Hanson $\&$ Puls (in prep. for A\&A), this is the consequence of a typical nebula-like situation, due to the competition between recombinations and downwards transitions, where the strong decay channel $(4 \rightarrow 3)$ (stronger than $5 \rightarrow 4$ itself) is of particular importance.

As shown in Fig. 21 (right), the emission component of $\mathrm{Br}_{\alpha}$ (and the line wings!) react strongly on $\dot{M}$, particularly for very weak winds (see also Najarro et al 
1998). In fact, if we now investigate the same $\dot{M}-L_{x}$ combinations as in Fig.20]using $\mathrm{Br}_{\alpha}$, we obtain a really promising result. From Fig. 22 we note that $\mathrm{Br}_{\alpha}$ reacts to changes in $\dot{M}$ even at lowest values. Evidently, for such low values (with a deepseated line formation region), the line profile is basically not affected by X-rays. With increasing mass-loss rate, however, the line starts to react on changes both in $\dot{M}$ and X-rays, due to an outwards moving line formation zone, reaching the layers to which X-rays can penetrate. This is clearly seen in Fig. 22 from the strong reaction of the He II emission component of $\mathrm{Br}_{\alpha}$, which is caused by the large sensitivity of the He II/He III ionization equilibrium to X-rays in the region where the emission arises. Our models indicate that under "normal" X-ray luminosities $\left(L_{\mathrm{x}} / L_{\mathrm{bol}}<10^{-4}\right)$ the (hydrogen) core of $\mathrm{Br}_{\alpha}$ is not affected though.

We thus expect that $\mathrm{Br}_{\alpha}$ will become the primary diagnostic tool to measure very low mass-loss rates at unprecedented accuracy, thus allowing to clarify whether they actually lie well below the theoretical predictions and to which degree.

\section{Inhomogeneous winds}

In our discussion of diagnostical methods (Sect.4.1) and recent results (Sect. 4.2) we have encountered a multitude of findings which are difficult or even impossible to reconcile with the standard model of a smooth and stationary wind. Even the inclusion of rotation and magnetic fields does not help to explain additional features, at least not at first glance. In the following, we will summarize various observational findings (ordered from low to high energies) which point towards a structured and variable wind. Further evidence will be outlined in Sect. 6.3 where we will concentrate on wind-clumping, maybe the most important aspect with respect to deviations from the standard model.

\subsection{Observational background}

Non-thermal radio emission has been discussed on page 40. According to recent investigations, the chances are high that this emission might be related to colliding winds alone. Insofar, we refrain here from considering non-thermal emission as an indication for shocks being present in the wind, in contrast to previous reasoning by various authors (including ourselves).

Electron-scattering wings of recombination lines in Wolf-Rayet stars are weaker than predicted by smooth models, indicating the presence of clumping (Hillier 1991). Due to a routine inclusion of the latter process into recent spectroscopic analyses of WR-stars, perfect fits have been obtained for (almost) all lines, and the derived mass-loss rates are a factor of two to three lower than if derived by means of unclumped models. Note that this diagnostics cannot be used in OBstars, due to the much lower wind-density and the corresponding lower optical depth in electron-scattering.

Optical line profile variability has been detected in the wind lines of OB-stars $\left(\mathrm{H}_{\alpha}\right.$ : Markova et al 2005 and references therein; He II 4686: Grady et al 1983, Henrichs 1991) and BA-supergiants (Kaufer et al 1996). Typical time-scales range from few 
hours/days (wind-flow times) to several months. Direct evidence for outward accelerating wind inhomogeneities (clumps) is implied by the "moving bumps" superimposed on the emission lines of Wolf-Rayet stars (e.g., Robert 1994), whilst similar evidence for O-stars has been reported for only few objects (see Sect.6.3).

Not only wind lines, but also lines which form to a significant or major part in the photosphere (e.g., He I $\lambda$ 5876) are variable. Fullerton et al (1996, and references therein) studied a large sample of O-stars and found that almost $80 \%$ of the investigated stars (all supergiants and a few late-type dwarfs) displayed photospheric line profile variability, with an amplitude increasing with stellar radius and luminosity. These findings were interpreted as being likely related to strange-mode oscillations (page 59), linking photospheric variability and structure formation in the wind.

Spectropolarimetric variations were detected by Lupie and Nordsieck (1987) in a sample of $10 \mathrm{OB}$ supergiants which pointed to the presence of clumps. Given that the position angles of the polarization, and thus the orientations of the electron scattering plane, were found to vary randomly, this implied that the polarization changes were not attributable to an orderly interaction with a companion, but the data provided direct evidence for the presence of an inhomogeneous wind structure.

Black troughs in saturated UV P Cygni profiles strongly point towards the presence of multiply non-monotonic flows, and can be simulated in stationary models only if allowing for a supersonic velocity dispersion (increasing with distance from the star) as the dominating line-broadening agent (page 36).

UV line profile variability is evident in UV P Cygni lines, with large variations of the blue edge, contrasted to an almost stationary red emission part (Henrichs 1991, Prinja 1992), implying a significant fraction of small-scale structure.

Discrete absorption components (DACs) detected in the absorption troughs of unsaturated P-Cygni profiles are the most intensively studied indicators of wind structure and variability. To date, they are believed to be formed in co-rotating interaction regions (CIRs) and will be discussed in Sect. 6.4, together with the so-called "modulation features".

$X$-ray emission from hot stars has been measured mostly at soft energies ( 0.1 to $\gtrsim 2 \mathrm{keV}$ ), either at low resolution in the form of a quasi-continuum or at high resolution allowing to see individual lines. The latter will be covered in the last part of this section (6.5).

Already the first X-ray satellite observatory, EINSTEIN, revealed that O-stars are soft X-ray sources. Follow-up investigations, mostly by ROSAT, have confirmed this notion and also allowed for quantifying the X-ray properties for a multitude of OB-stars (see Kudritzki and Puls 2000 and references therein). Roughly, the (soft) X-ray luminosity scales with $L_{\mathrm{x}} \approx 10^{-7} L_{\mathrm{bol}}$. First there appeared to be a rather large scatter indicating the influence of additional parameters, however a more recent XMM-NEWTON study by Sana et al 2006 of the young open cluster NGC 6231 showed that the majority of O-stars display a very limited dispersion (of 40 or only 20 per cent depending on whether cool dwarfs are included or not). This suggests that the intrinsic X-ray emission of normal O-stars is rather constant for any given object and that the level of X-ray emission is accurately related to the basic stellar and wind parameters. 
The source of the X-ray emission is widely believed to be shocks embedded in the stellar wind and related (though not necessarily directly, see Sect. 6.2) to the line-driven instability. In terms of a stationary description 28 , a simple model (e.g., Hillier et al 1993, Cassinelli et al 1994) assumes randomly distributed shocks where the hot shocked gas (with temperatures of a few million Kelvin and a volume filling factor of the order of $10^{-3} \ldots 10^{-2}$ in the case of denser O-type winds) is collisionally ionized/excited and emits X-ray photons due to spontaneous decay, radiative recombinations and bremsstrahlung. The ambient "cool" stellar wind (with a kinetic temperature of the order of $T_{\text {eff }}$ ) can re-absorb part of the emission due to $\mathrm{K}$ - and L-shell processes if the corresponding optical depths are large. This simple model (sometimes extended to account for the different post-shock cooling zones of radiative and adiabatic shocks, see Feldmeier et al 1997) has been used to analyze large samples of O-stars observed with ROSAT (Kudritzki et al 1996), and is still used in the context of NLTE diagnostics to account for the influence of X-ray/EUV emission on the photo-ionization rates (see below). A simple scaling analysis of the involved X-ray emission and absorption processes by Owocki and Cohen (1999) showed that the "natural" scaling for optically thin (with respect to X-ray absorption) winds is given by $L_{\mathrm{x}} \propto\left(\dot{M} / v_{\infty}\right)^{2}$ and for optically thick winds by $L_{\mathrm{X}} \propto\left(\dot{M} / \nu_{\infty}\right)^{1+s}$, if the volume filling factor follows a radial stratification $f \propto r^{s}$. This would imply, for O-stars, $s \approx-0.4$ to explain $L_{\mathrm{x}} \propto L_{\text {bol }}$ by means of the scaling relations for line-driven winds (Eqs. 13, 14 ).

Whereas ROSAT observations of OB-star winds showed correlations between $L_{\mathrm{X}}$ and $L_{\text {bol }}$, WC stars were not found to emit in the X-ray regime, unless the star is part of a binary system and the X-ray emission can be attributed to colliding winds. The fact that single WC stars (for WN stars the situation is less clear) are no significant X-ray emitters does not imply that shocks do not develop in their winds. In fact, Ignace and Oskinova (1999) could explain the lack of X-ray emission in single WR winds by realizing that the wind is optically thick to the hot X-ray gas. The absence of X-ray detections in single WC stars was reinforced by more sensitive XMM-NEWTON and CHANDRA data, where the lack of X-ray emission was attributed to photoelectric absorption by the wind and where the large opacity of the WR winds was thought to put the radius of optical depth unity at hundreds or thousands of stellar radii for much of the X-ray band (Oskinova et al 2003).

Since the X-ray and associated EUV luminosity emitted by the shocks is quite strong, it can severely affect the degree of ionization of highly ionized species such as CV, Nv and Ovi, by Auger-ionization (Macfarlane et al 1993) and even more by direct ionization in the EUV (Pauldrach et al 1994). In fact, the so-called superionization in the winds of $\mathrm{O}$ supergiants (exemplarily, the strength of the OVI resonance line in $\zeta$ Pup, see Sect.4.1) can be explained only by means of this additional source of photons. A systematic investigation of these effects on the complete FUV spectrum, as a function of stellar parameters, mass loss and X-ray luminosity has been performed by Garcia (2005).

Correlated variability. Finally, let us note that both $\mathrm{H}_{\alpha}$ and UV variability (Kaper et al 1997) and $\mathrm{H}_{\alpha}$ and $\mathrm{X}$-ray variability (Berghoefer et al 1996) have been found to be correlated, which indicates the propagation of disturbances throughout the entire wind.

\footnotetext{
${ }^{28}$ Generally, the X-ray luminosity of O and early B-stars is constant to within 10 to $20 \%$, see
} Berghoefer and Schmitt 1994. 
6.2 Theoretical background: The line-driven instability and time-dependent models

Historically, most of the theoretical effort to explain the above observational features has concentrated on the line-acceleration itself. Later on, however, it turned out that at least one of the primary indicators of time-dependence and structure, the DACs, can be understood within the concept of CIRs, rather (but not completely) independent on the specific physics of the wind acceleration (for details, see Sect.6.4).

Linear stability analysis. Already in their pioneering paper on line-driven winds, Lucy and Solomon (1970) pointed out that the accelerating mechanism of these winds should be subject to a strong instability, and subsequent investigations tried to quantify its properties, at first based on linear stability analyses. By linearizing the time-dependent equations for density and momentum in a frame comoving with the mean fluid and using a planar approximation with height co-ordinate $z$, it is easy to show that the conventional ansatz

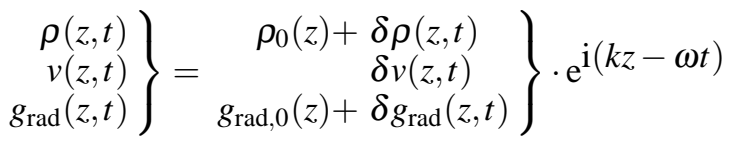

for the density, velocity and line force (quantities with subscript ' 0 ' refer to the mean flow, $v_{0}=0$, and $\delta \rho, \delta v, \delta g_{\text {rad }}$ are the corresponding amplitudes of a sinusoidal disturbance with wavenumber $k$ and frequency $\omega$ ) results in

$$
\left.\begin{array}{rl}
-\mathrm{i} \omega \delta \rho+\rho_{0} \mathrm{i} k \delta v & =0 \\
-\mathrm{i} \omega \delta v & =\delta g_{\mathrm{rad}} \quad \Rightarrow \quad \frac{\delta v}{\delta \rho}=\frac{1}{\rho_{0}} \frac{\omega}{k} \\
\delta & =\mathrm{i} \frac{\delta g_{\mathrm{rad}}}{\delta v}
\end{array}\right\} \quad \frac{\delta v}{\delta \rho}=\frac{\mathrm{i}}{\rho_{0} k} \frac{\delta g_{\mathrm{rad}}}{\delta v}
$$

In the latter equation, we have neglected the pressure terms (for a complete analysis, see Owocki and Rybicki 1984). Thus, the phase angle between density and velocity disturbance, $\cos \varphi$, the growth-rate of the instability, $\Omega$, and its phase speed with respect to the mean flow, $v_{\varphi}$,

$$
\cos \varphi=\frac{-\mathfrak{I}\left(\delta g_{\mathrm{rad}} / \delta v\right)}{\left.\mid \delta g_{\mathrm{rad}} / \delta v\right) \mid}, \quad \Omega=\mathfrak{I}(\omega), \quad v_{\varphi}=\mathfrak{R}(\omega) / k
$$

are completely determined by the response of the line-acceleration on the velocity disturbance, at least in the supersonic regime where the neglect of the pressure force is justified $(\mathfrak{R}(x)$ and $\mathfrak{I}(x)$ are the real and the imaginary part of the $x$ quantity, respectively). Thus, a correct description of the line force is of highest importance for time-dependent simulations (see below).

Within the Sobolev approximation 29 (page 7) it turns out that $\delta g_{\mathrm{rad}}^{\mathrm{Sob}} / \delta v \propto \mathrm{i} k$, i.e., it is purely imaginary. Consequently $\cos \varphi=-1$ and density and velocity are completely anti-correlated. There is no growth of the instability, $\Omega^{\mathrm{Sob}}=0$, but only an oscillatory behaviour, and the phase speed (independent of $k$, i.e., the

\footnotetext{
29 Remember that most stationary wind models are based on this approach.
} 
medium is non-dispersive in this approximation) is inwards directed. The inclusion of pressure terms leads to slight modifications: the inwards directed phasespeed becomes marginally larger (roughly by $a^{2} / v_{\varphi}$ ), and a slow, outwards propagating mode becomes possible, at a speed $\approx a^{2} / v_{\varphi}<a$ (Abbott 1980).

The (absolute) value of $v_{\varphi}$ (inwards mode) at the critical point (page 9) of stationary models based on a Sobolev line-force turns out to be identical with the corresponding flow speed, much larger than the speed of sound. Insofar, this critical point may be considered as a critical point in the "usual" sense (Holzer 1977): It is the outermost point in the flow where a communication with lower layers is possible, in this case via the above radiative-acoustic waves which have been named as Abbott-waves according to their "inventor". Following Holzer's "windlaws", $\dot{M}$ then depends solely on processes in the sub-critical region, whilst $v_{\infty}$ is controlled by the acceleration in the super-critical part. For a detailed discussion of Abbott-waves and the corresponding modifications of the above wind-laws for line-driven wind we refer the reader to Feldmeier and Shlosman (2002). In any case, however, remember that the use of the Sobolev approximation is justified only for wavelengths which are large compared with the Sobolev length (page 7), $L^{\mathrm{Sob}}=v_{\mathrm{th}} /(\mathrm{d} v / \mathrm{d} r)$, i.e., for small $k$. A discussion of critical points in models based on non-Sobolev line-forces is beyond the scope of this review.

A somewhat opposite approach (MacGregor et al 1979, Carlberg 1980) results in a purely real response, $\delta g_{\text {rad }}=A \delta v$, with $\mathfrak{I}(A)=0$ and $A>0$, which is valid as long as the disturbances remain optically thin. In this case, the finite sound-speed is of importance and disturbances can propagate only for large $k$, in both directions and becoming strongly amplified, with a phase-speed of the order of $a$.

A combination and generalization of these two alternative approaches has been obtained by Owocki and Rybicki (1984) in the form of a "bridging-law", which results from a perturbation analysis of the line-force. In this case, $\delta g_{\mathrm{rad}} / \delta v$ has both a real and an imaginary component, and includes the two limiting cases for small and large $k$ from above 30 The resulting phase relation between density and velocity is predominantly anti-correlated for all $k$, and there is a fast, exponential growth of the inwards propagating modes, within a dispersive medium. Important additions to this analysis have been provided by Lucy (1984) and Owocki and Rybicki (1985), by considering the effects of the disturbed diffuse radiation field and associated line-force (the so-called "line drag' 31 ) which had been neglected in the previous approaches.

Time-dependent models. After these initial studies, effort has concentrated on a direct, hydrodynamical modeling of the time-dependent structure of line-driven winds (for reviews, see Owocki 1994c and Feldmeier 1999). As already pointed out, the key aspect concerns a consistent description of the line force which follows the local and non-local conditions (due to the coupling with the radiation field) at all scales of structure formation. In order to keep the problem computationally feasible, an "exact" calculation of the line-force is prohibitive, and

\footnotetext{
30 The critical length scale is here the "bridging length" $\chi_{\mathrm{B}}^{-1}$, with $\chi_{\mathrm{B}}$ the line opacity required to reach unit optical depth.

31 The disturbed diffuse radiation field in resonance lines leads to a damping effect which, at the foot-point of the wind, exactly cancels and otherwise diminishes the instability resulting from pure absorption lines.
} 
complex integral forms of various degrees of approximation have been developed (Owocki and Puls 1996, 1999) to allow for this objective.

After incorporating the stabilizing effect of the line drag by the diffuse, scattered radiation field (see above), hydrodynamical models have proven to be significantly more stable in the lower wind than the first ones based on pure absorption (Owocki et al 1988). The outer wind $\left(r \gtrsim 1.3 R_{*}\right)$, however, still develops extensive structure that consists of strong reverse 32 shocks separating slower, dense material from high-speed rarefied regions in between. This is a direct consequence of the strong amplification of the inwards propagating mode with a predominant anti-correlation of density and velocity, as derived from the linear analysis. Such structure is the most prominent and robust result from time-dependent modeling, and the basis for our interpretation and description of wind-clumping. Moreover, the multiply non-monotonic nature of the velocity field explains the black absorption troughs observed in saturated UV P Cygni lines in a natural way (Lucy 1982, 1983, Puls et al 1993a, Owocki 1994c).

Feldmeier (1995) extended these models by accounting for the energy transport including radiative cooling. By investigating the impact of various photospheric disturbances which can affect the on-set of structure formation (via exciting the line-force instability), Feldmeier et al (1997) concluded that clump-clump collisions (and not the cooling of the reverse shocks themselves, as previously suspected, since this results in much too low filling factors) are the origin of the observed X-ray emission.

Recent effort has focused on more-dimensional simulations and on the study of the outermost wind. By means of an efficient 1-D "pseudo-periodic box formalism" to investigate the evolution at distances very far from the central star, Runacres and Owocki (2005, see also Runacres and Owocki 2002) showed that structure can survive out to distances of more than 1000 stellar radii, due to supersonic clump-clump collisions which counteract the pressure expansion.

Initial results of 2-D simulations have been obtained using different approximations for the calculation of the line-force. In the simplest case (radiatively isolated azimuthal zones and neglect of lateral line-drag 33 ), the shells arising in 1-D models are broken up by Rayleigh-Taylor or thin-shell instabilities, resulting in a completely incoherent lateral structure (Dessart and Owocki 2003). A follow-up investigation (Dessart and Owocki 2005) showed that by accounting for the lateral line-drag and the lateral mixing in a more self-consistent manner (three rays at different impact parameters, as already used by Owocki 1999), such models - at least at highest resolution - show a much larger lateral coherence than corresponding one-ray models. Quantitative results are still lacking though. Further effort is needed to answer the important question about the lateral and radial length scale of clumps, required to check and improve present assumptions when modeling the spectra from the IR to the X-ray regime.

Time-dependent versus stationary approach. Obviously, time-dependent models based on a non-local, unstable line-force are able to explain a large number

\footnotetext{
32 Reverse shocks travel backwards in the comoving frame (though outwards in the stellar frame). In the pre-shock region (starwards from the shock), the velocity is high and the density low, and vice versa in the post-shock region.

33 see Rybicki et al (1990).
} 
of observational findings, at least qualitatively. On the other hand, they appear to be in stark contrast with our assumptions within the standard model (Sect. 2.1), both with respect to modeling and diagnostics. However, when viewed with respect to the mass distribution of density and velocity, the stationary and timedependent models are quite similar (e.g., Fig. 32, left panel). Given the intrinsic mass-weighting of spectral formation, this explains why detailed line-synthesis calculations assuming line-opacities proportional to the local density are able to generate (time-dependent) line profiles quite similar to observations (Puls et al 1993a).

Let us also emphasize that the gross wind properties derived from time-dependent models like the terminal flow speed and time-averaged mass loss rate agree well with those following from a stationary approach. Deviations from this rule due to source function gradients in the transonic region (Owocki and Puls 1999, see also Sect. 5) are not a consequence of the line-driven instability. Instead, they originate from an incorrect use of the local, Sobolev approach in the stationary models in a region where this approach is no longer justified, mainly because of the strong curvature of the velocity field 34

\subsection{Wind clumping}

In our review of "observed" wind parameters (Sect. 4), we have frequently referred to the process of wind-clumping, and particularly to its impact on the derived mass-loss rates, without providing further details. Indeed, the investigation of this process and its consequences constitutes a major fraction of studies performed in the last decade, and various contributions regarding the present statusquo can be found in the proceedings of a recent workshop dedicated to this topic (Hamann et al 2008b).

In the previous two sections, we have summarized important observational and theoretical findings which unambiguously indicate that radiation driven winds are structured and time-variable, and we have outlined that the line-driven instability is a primary candidate for explaining at least part of these findings. Thus, we are now in a position to consider wind-clumping in more detail, starting with a discussion of more specific evidence.

\subsubsection{Observational evidence}

Half a century of clumping. The presence of clumping as "extreme density fluctuations" around hot stars was first invoked by Osterbrock and Flather (1959) to explain the discrepancy between observed and predicted fluxes in the $\mathrm{H}$ II region in the Orion nebula. Two decades later, Lucy and White (1980) proposed a phenomenological model to explain the observed X-ray luminosity of $\zeta$ Pup, in which the stellar wind would break up into a population of radiatively driven blobs and where the radiation should originate from the bow shocks preceding the blobs (see also Sect.6.5.2). Applying the same formalism as Osterbrock and Flather (1959),

\footnotetext{
34 Remember that standard Sobolev theory requires a vanishing curvature over the corresponding Sobolev length, which is small in the transonic region.
} 
Abbott et al (1981b) investigated the effects of dense clumps on the thermal freefree radio emission of stellar winds as a function of both the volume filling factor $\left(f_{\mathrm{vol}} \leq 1\right)$ and the density ratio between clumped and inter-clump matter. For the standard assumption of vanishing inter-clump density (see below), they showed that the radio flux is a factor of $f_{\mathrm{vol}}{ }^{-2 / 3}$ larger than that from a homogeneous wind with the same average mass-loss rate. Thus, using Eq. 45, radio mass-loss rates derived from clumped winds are lower by a factor of $f_{\mathrm{vol}}{ }^{1 / 2}$ compared to unclumped winds.

Wind-clumping in dense winds. In the mid-80s, two more observational diagnostics suggested that the standard, and convenient, assumption of homogeneous stellar winds might fail due to clumping. The first line of evidence came from studies of the light-curve eclipses as a function of wavelength in the $\mathrm{WR}+\mathrm{O}$ binary V444 Cyg (Cherepashchuk et al 1984). The differences in shape and depth of eclipses from the UV to the IR could not be simultaneously interpreted using a smooth electron density distribution in the wind. The authors required the presence of individual density condensations becoming optically thick to reconcile the electron scattering $(\propto \rho)$ dominated UV and optical fluxes with the free-free and bound-free $\left(\propto \rho^{2}\right)$ infrared values.

The second line of evidence relied on studies of continuum and line variability. For continuum investigations, linear polarization variability is particularly advantageous. This process, being directly related to the electron density through electron scattering processes, provides information on the presence of localized inhomogeneities and is more reliable than pure continuum photometric variability studies, where other processes such as rotational modulation or pulsations may come into play. In addition to the OB-star work by Lupie and Nordsieck (1987), mentioned already in Sect.6.1, extensive and systematic monitoring of linear polarization in WR-stars (St.-Louis et al 1987) and LBVs (Taylor et al 1991, Davies et al 2005) revealed stochastic variability on short time scales, indicating that the material had to be confined in clumps. More recent results helping to quantify the clumping properties are discussed below.

In the case of lines, time-resolved spectroscopic monitoring of WR-stars (Moffat et al 1988, Robert 1994) revealed the presence of narrow emission features on top of the broad emission lines that appeared to propagate from the line centers to the line wings on timescales of the wind flow-time. These moving sub-peaks were interpreted as evidence for blob ejections into the wind and are now considered to be one of the most clear-cut evidences for clumping in WR winds.

Lepine and Moffat (1999) monitored the line-profile variations in the He II emission lines of WN stars and in C III emission lines of WC stars, and studied these variations by means of a detailed wavelet analysis. As well, these authors performed simulations of line-profile variability (lpv) assuming a wind consisting of a large number $\left(\sim 10^{4}\right)$ of discrete wind-emission elements (DWEEs). Observations were best reproduced by assuming a large ratio of the radial to the lateral velocity dispersion for the DWEEs, consistent with the nature of the line-driven instability and the corresponding lateral line-drag (page 70). Physical models of such lpv interpreted in terms of clumps generated by the line-driven instability have been provided by Dessart and Owocki (2002a,b), where the latter study extends the Lepine and Moffat (1999) wavelet formalism to modeling lpv from hy- 
drodynamic simulations. A key result is that one can fit most of the characteristics if one assumes a lateral coherence scale of $\sim 3$ degrees.

Wind-clumping in OB-star winds. For O-stars, observational lpv-studies are more difficult due to their roughly factor of ten thinner winds. First investigations were carried out by Eversberg et al (1998) on the He II 4686 line in $\zeta$ Pup, which revealed outward-moving inhomogeneities in its wind that started near the stellar surface and became invisible at $\sim 2 R_{*}$. From the wind acceleration, the authors derived a value of $\beta=1$, consistent with results from independent diagnostics assuming unclumped winds. Thus, it seems that the clumping properties of O- and WR stars are similar, and this "universality" has been recently confirmed by Lepine and Moffat (2008) who extended the study to five hot stars in different evolutionary stages. They show that the moving sub-peaks identified on top of the broad emission lines in WR-stars appear also in the Of stars $\zeta$ Pup and HD 93129A, in the more evolved hydrogen-rich and luminous Of-like WN stars HD 93131 and HD 93162, and in the more mass-depleted WC star in $\gamma^{2}$ Vel. The authors conclude that stochastic wind clumping is a universal phenomenon in the radiation-driven, hot winds from all massive stars.

Markova et al (2005) presented a study on the lpv of $\mathrm{H}_{\alpha}$ for a sample of 15 O-type supergiants, ranging from $\mathrm{O} 4$ to O9.7. All stars showed evidence of significant lpv, mostly dominated by processes in the wind, where the variations were found to occur from the wind base on out to $\sim 0.3 v_{\infty}$. From profile simulations, they concluded that the mass-loss rate is only marginally variable, resulting in insignificant variations of the wind momenta and hence of the WLR.

Of course, best direct evidence for the presence and behaviour of clumps in stellar winds would be reached using imaging devices with enough spatial resolution to "see" the individual clumps close to the star and track them as they expand with the wind flow. By such imaging, also geometric constraints on, for instance, the sizes of the clumps might be obtained (e.g. Chesneau et al 2000). Unfortunately, micro-arcsec resolution would be required to carry out such studies, even for the nearby O-stars. Present observing facilities allow to see such clumps only at much larger scales, such as the outer wind regions where the thermal radio emission originates from (Williams et al 1997). However, although these observations indicate that clumps seem to survive over the entire stellar wind (see also Runacres and Owocki 2005), no information is provided on their formation region nor how they evolve throughout the wind. It should be noted that even after the possible commissioning of 100 -m class telescopes, one would still not be able to spatially resolve the innermost wind regions where the clumps originate, and it is thus pivotal that alternative methods to constrain clumping properties are further developed, such as linear polarimetry (see below).

At least for O-stars, however, we presently have to rely mostly on indirect methods to address the behaviour of clumping throughout the stellar wind. Such indirect evidence for the presence of clumping was found in parallel by Markova et al (2004) and Repolust et al (2004). By means of a comparison between the observed and predicted WLR for large samples of Galactic O-stars, these authors showed that if the stellar sample was plotted as a function of $\mathrm{H}_{\alpha}$ profile type (stars with $\mathrm{H}_{\alpha}$ in emission and those with $\mathrm{H}_{\alpha}$ in absorption, partly filled in by wind emission), two different WLRs appeared, inconsistent with the basic theoretical 
prediction that the wind-momentum rates of O-stars should depend on luminosity alone (Sect. 2] and Fig. [5). It was suggeste 23 that this difference is related to windclumping (being "visible" only in denser winds, i.e., in those with $\mathrm{H}_{\alpha}$ in emission) and shown that a unification of both "observed" WLRs with the theoretical one is possible when the average clumping factor, $f_{\mathrm{cl}}\left(=f_{\mathrm{vol}}{ }^{-1}\right.$, see below) of the clumped matter in stars with $\mathrm{H}_{\alpha}$ in emission is of the order of $f_{\mathrm{cl}} \approx 5$, implying a net reduction of $\dot{M}$ by a factor of 2.3. Note that these values differ significantly from those derived by means of UV studies with net $\dot{M}$ reductions up to factors of 10 (e.g., Bouret et al 2003, Fullerton et al 2006, see below).

Therefore, at this stage, several fundamental questions need to be addressed:

- Which are the upper and lower limits for the clumping factor obtained from observationally driven studies and how reliable are they?

- What is the stratification of the clumping factor throughout the wind, and what is the shape of the clumps?

- How realistic is the treatment of clumping assumed so far and how well can modern, sophisticated atmospheric codes account for it?

- How much mass-loss reduction can evolutionary models of massive stars cope with to still be consistent with independent constraints such as the observed numbers of WR-stars?

Before we can consider these points in detail, we have to describe the basic concept underlying most of the following analyses.

"Micro-clumping". This concept bases on the hypothesis that the wind consists of small-scale density inhomogeneities, which redistribute matter into overdense clumps and underdense inter-clump matter. Motivated by the principal results from hydrodynamical simulations including the line-driven instability, the inter-clump matter is assumed to be void ${ }^{36}$ In this case then, the average density $\langle\rho\rangle=\dot{M} /\left(4 \pi r^{2} v\right)$ can be described by

$$
\langle\rho\rangle=f_{\mathrm{vol}} \rho^{+}, \quad\left\langle\rho^{2}\right\rangle=f_{\mathrm{vol}}\left(\rho^{+}\right)^{2}
$$

where $\rho^{+}$is the density inside the overdense clumps, and $\left\langle\rho^{2}\right\rangle$ is the mean of the squared density. Thus, the clumping factor as introduced by Owocki et al (1988),

$$
f_{\mathrm{cl}}=\left\langle\rho^{2}\right\rangle /\langle\rho\rangle^{2} \Rightarrow f_{\mathrm{cl}}=f_{\mathrm{vol}}^{-1} \quad \text { and } \rho^{+}=f_{\mathrm{cl}}\langle\rho\rangle,
$$

corresponds to the inverse of the volume filling factor and measures the overdensity of the clumps. Since we assume void inter-clump material, matter is present only inside the clumps, with density $\rho^{+}$, and the corresponding opacity is given by $\kappa=\kappa_{C}\left(f_{\mathrm{cl}}\langle\rho\rangle\right)$, where $C$ denotes the process evaluated inside the clump. Optical depths have to be calculated via $\tau=\int \kappa_{C}\left(f_{\text {cl }}\langle\rho\rangle\right) f_{\text {vol }} \mathrm{d} r$, with a reduced path length $\left(f_{\text {vol }} \mathrm{d} r\right)$ to correct for the volume where a clump is "seen". Note that this

\footnotetext{
35 following a previous idea by Puls et al (2003).

36 Alternatives from this assumption are rare, and not used in standard diagnostical tools until recently when Zsargó et al (2008a) re-investigated the "superionization" of O vi (page 67) in $\zeta$ Pup. Their simulations show that clumped wind models that assume a void interclump medium cannot reproduce the observed O VI profiles, though enough O VI can be produced if the voids are filled by a low-density gas.
} 
expression is valid only if all clumps are optically thin. Extensions for optically thick clumps are given in Sect.6.3.3. Combining these results, optical depths for a clumped medium can be expressed by a mean opacity, $\bar{\kappa}$,

$$
\tau=\int \bar{\kappa} \mathrm{d} r \quad \text { with } \quad \bar{\kappa}=\kappa_{C}\left(f_{\mathrm{cl}}\langle\rho\rangle\right) f_{\mathrm{vol}}=\frac{1}{f_{\mathrm{cl}}} \kappa_{C}\left(f_{\mathrm{cl}}\langle\rho\rangle\right) .
$$

Thus, for processes that are linear in density, the mean opacity of a clumped medium is the same as in the homogeneous case, whereas for processes scaling with the square of density, mean opacities (and emissivities) are effectively enhanced by a factor $f_{\mathrm{cl}}$. Moreover, radiative processes described by the microclumping approach do not depend on the size, the geometry or the distribution of the clumps, but on the radial stratification of the clumping factor alone. The enhanced opacity for $\rho^{2}$ dependent processes has the consequence that mass-loss rates derived by such diagnostics are lower by a factor of $\sqrt{f_{\mathrm{cl}}}$ than corresponding mass-loss rates assuming no clumping. Consequently, the optical depth invariant, $Q$ (see Eq. 39), for such processes might be extended according to

$$
Q=\frac{\dot{M} \sqrt{f_{\mathrm{cl}}}}{\left(R_{*} v_{\infty}\right)^{1.5}}=\frac{\dot{M}}{\sqrt{f_{\mathrm{vol}}}\left(R_{*} v_{\infty}\right)^{1.5}}
$$

as long as there is no strong radial variation of $f_{\mathrm{cl}}$ or $f_{\mathrm{vol}}$.

\subsubsection{Quantification of wind-clumping}

Let us now review how observations and models have evolved during the last decade and summarize the most important results concerning the "chase" of the clumping factor.

One of the first (and still most important) attempts to quantify wind-clumping was carried out by Hillier (1991) who analyzed the corresponding effects on the $\rho^{2}$-dependent emission components and the $\rho$-dependent electron scattering wings in WR emission lines. Noting that standard, homogeneous models severely overestimated the electron scattering wings of the observed profiles, Hillier (1991) showed how both components could be simultaneously reproduced when clumping is accounted for and the mass-loss rate is reduced by a factor of $\lesssim 2$.

For the method to work, the electron scattering wings have to be strong and clearly defined, i.e., a good continuum rectification is required. The method also assumes that the strength of the $\rho^{2}$-dependent emission can be described by microclumping alone. Thus, this method may also be applied to "well behaved" LBVs like P Cygni, for which Najarro (2001) found $f_{\mathrm{vol}} \approx 0.5$ by analyzing all the Balmer lines.

Results using the new generation of unified model atmospheres accounting for blanketing and clumping started to appear with the new millennium (see Sect. 4.2.17. From quantitative IR studies in the Galactic Center, Figer et al (2002) found

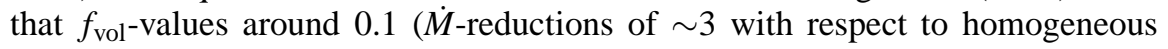
models) were required to best reproduce the spectra of WNL and Of stars in the Arches Cluster. Analyses carried out for $\mathrm{O}$ supergiants in the Magellanic Clouds (Crowther et al 2002, using UV and optical data) revealed that $f_{\text {vol }}=0.1$ could reproduce the observed $\mathrm{P} \vee \lambda \lambda$ 1118-1128 doublet without requiring a reduced phosphorus abundance as suggested by Pauldrach et al (1994, 2001). Further analyses 

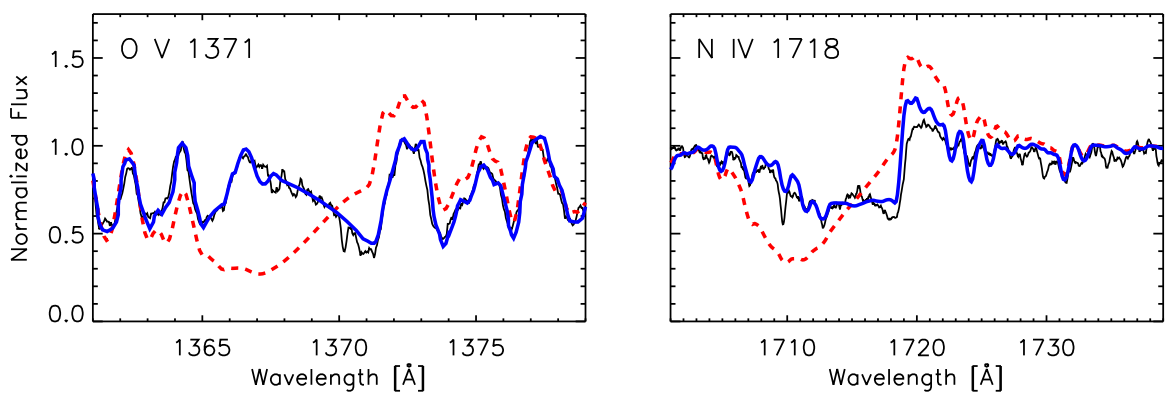

Fig. 23 Effects of clumping and a correspondingly reduced mass-loss rate in the UV O v $\lambda 1371$ and N IV $\lambda 1718$ lines of HD 96715 (O4((f))). The derived $f_{\text {vol }}=0.02$ value implies a factor of 7 reduction in $\dot{M}$. Dotted: Profiles from an unclumped model which reproduces other, clumpinginsensitive lines equally well. Adapted from Bouret et al (2005).

in the SMC found likewise $f_{\text {vol }}$ values around 0.1 (Hillier et al 2003) and lower $\left(f_{\mathrm{vol}}=0.1-0.01\right.$ in four O stars of the SMC cluster NGC346, Bouret et al 2003). A similar study was carried out using UV (FUSE + IUE) spectra of two Galactic $\mathrm{O} 4$ stars by Bouret et al (2005). In this case they found that values as low as $f_{\mathrm{vol}}=$ 0.02 and 0.04 were required to satisfactorily reproduce not only the $P \vee \lambda \lambda 1118$ 1128 and the $\mathrm{O} \vee \lambda 1371$ lines, but also N IV $\lambda 1718$, as shown in Fig. 23. Though indicating an enormous degree of clumping (overdensities of factors between 25 to 50!), these numbers are consistent with the alternative explanation in terms of a strong oxygen depletion (factor 50) in early $\mathrm{O}$ stars, suggested by Pauldrach et al (2001) as a result of analyses using homogeneous models.

Interestingly, the lower $f_{\mathrm{vol}}$-values found for the SMC objects seem to correspond to the Galactic ones down-scaled by the abundance ratio. Major uncertainties in these UV-based methods are due to the role of X-rays and the validity of micro-clumping (see below) assumed for the models. Other effects such as changes in the ionization structure driven by clumping are included, however.

The $P$ v problem. Although the problem with the $\mathrm{O} \vee \lambda 1371$ line had been extensively debated in the literature (Haser et al 1998, Pauldrach et al 2001, Bouret et al 2003), it was the advent of the FUSE mission providing the cleaner $P \vee \lambda \lambda 1118$ 1128 mass-loss diagnostics that gave rise to the so called $\mathrm{P} v \mathrm{v}$ problem (see Fullerton et al 2008 for a comprehensive review of this issue). Due to the very low cosmic abundance of phosphorus, the $\mathrm{P} \vee \lambda \lambda 1118-1128$ doublet basically never becomes saturated, not even when $\mathrm{P}^{+4}$ is the dominant ion. This enables to obtain a direct estimate of the product $\dot{M}\langle q\rangle$, where $\langle q\rangle$ is a spatial average of the ion fraction of the species (cf. page 36). Unfortunately, estimates of $\langle q\rangle$ for any given resonance line is problematic unless detailed NLTE modeling is performed, and even then, problems such as X-rays or subtle blanketing/blocking effects may lead to incorrect predictions. On the other hand, an empirical determination of ionization fractions is not feasible, since access to resonance lines from consecutive ionization stages are not available in the far-UV and UV regions. In the case of $\mathrm{PV}$, some insight is gained from the available FUSE atlases of Galactic and Magellanic OB spectra 

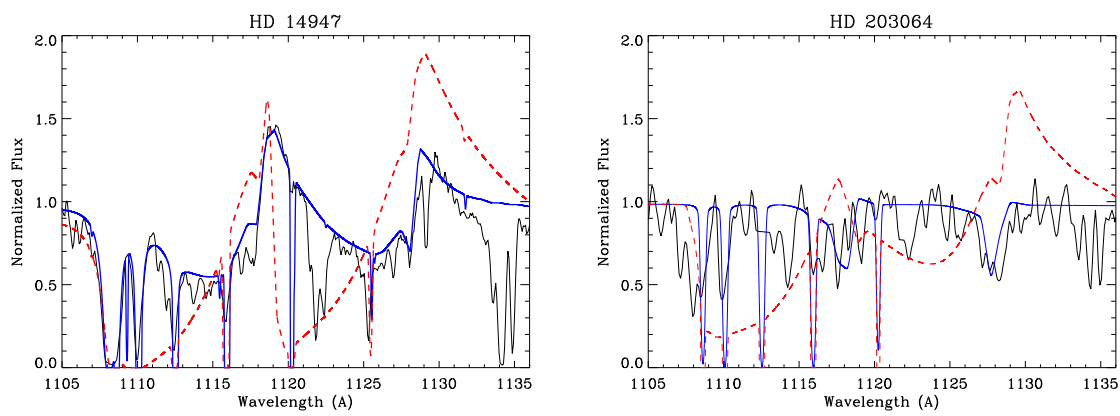

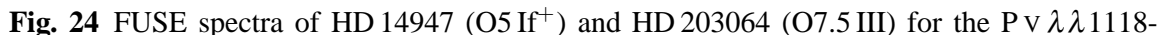
1128 doublet, together with the best fits from the simulations by Fullerton et al (2006) with a low $\dot{M}$ (solid) and the $\dot{M}$ derived from $\mathrm{H}_{\alpha}$ using homogeneous models (dashed). To unify

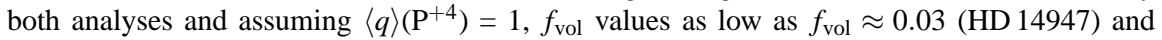
$f_{\mathrm{vol}} \approx 0.003$ (HD 203064) are required, resulting in a down-scaling of the "unclumped" massloss rates by factors of 6 and 18, respectively.

(Pellerin et al 2002, Walborn et al 2002). These show that the doublet is very weak for the earliest O-subtypes, exhibits a stable maximum over a wide range of spectral types, and then starts to disappear rather quickly for stars between $\mathrm{O} 9.5$ and B0. Such morphology seems to secure a maximum of the $\mathrm{P}^{+4}$ ionization fraction for mid O-type stars, a behaviour confirmed by model atmosphere calculations. Therefore, for those O-stars within a safe $\langle q\rangle \approx 1$ region, the $\mathrm{P} \vee \lambda \lambda 1118-1128$ line should provide a robust and independent estimate of $\dot{M}$ (pure $\rho$-diagnostic).

Fullerton et al (2006) carefully selected a sample of 40 Galactic O-stars, for which also reliable $\rho^{2} \dot{M}$-estimates $\left(\mathrm{H}_{\alpha}\right.$, radio) were available, and carried out a detailed comparison between both methods. Surprisingly (see Figs. 24 and 25) they found a severe discrepancy by a factor of $\sim 10$ in $M$ for mid-range O-stars and by substantially larger factors for earlier and later spectral types. A more restrictive consideration, taking into account only those stars for which $\langle q\rangle\left(\mathrm{P}^{+4}\right)$ is expected to be 1, i.e., the "safer" mid-range $\mathrm{O}$ supergiants, resulted in a median discrepancy $\dot{M}\left(\rho^{2}\right) /(\dot{M}(\mathrm{P} \vee)\langle q\rangle)=20$, which would imply $f_{\mathrm{cl}} \approx 400$ if interpreted in terms of micro-clumping, i.e., extreme clumping, much larger than derived by other methods. This discrepancy is the $\mathrm{PV}$ problem and is nicely illustrated in Fig.25. At the end of this section we present a possible solution of this problem.

Polarimetric studies. Soon after the detection of moving subpeaks on top of the broad emission lines, other indications for clumping of WR winds were found. Besides the diagnostics provided by the electron scattering wings, a straightforward comparison of mass-loss rates based on $\rho^{2}$-diagnostics with rates determined using other methods, such as those from polarization variations in $\mathrm{WR}+\mathrm{O}$ binaries 37 (e.g. St.-Louis et al 1993), suggested that the mass-loss rates determined from emission-lines are overestimated by about a factor three (Robert 1994), which

37 The basic principle is that the light from the O-star acts as a probe of the WR-wind via electron scattering. O-star photons scatter off the abundant free electrons in the WR wind, which polarizes the light received by the observer, where the level of polarization depends on the orbital-phase. 


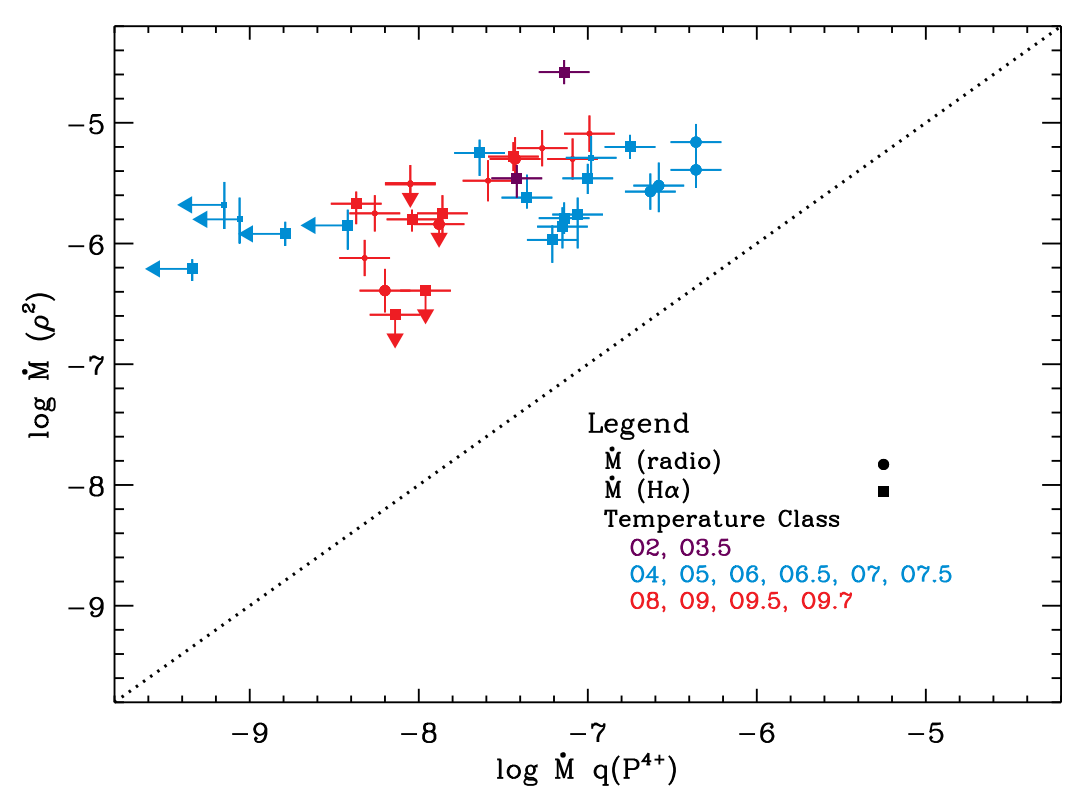

Fig. 25 Comparison of $\dot{M}$ (derived from $\rho^{2}$-diagnostics) with $\dot{M}\langle q\rangle\left(P^{+4}\right)$ for a large sample of Galactic O-stars. Adapted from Fullerton et al (2006), see text.

agrees with the factors of 2-4 derived from NLTE analyses using clumped models (e.g. Hamann and Koesterke 1998).

Extending the above technique, St.-Louis and Moffat (2008) studied the polarimetric variability of $\mathrm{O}$-stars winds in six massive $\mathrm{O}+\mathrm{O}$ binary systems. They reported clumping independent mass-loss rate estimates that suggest only small clumping corrections. However, the $\mathrm{O}$-stars analyzed by this method had relatively weak winds and, as a consequence, the electron scattering optical depth of the wind is low. Thus, a significant fraction of the polarization signal originates from scattering off the photosphere, implying that the derived mass-loss rates should be lowered.

In their investigation of the polarization variability in WR-winds, Robert et al (1989) found an anti-correlation between the terminal wind velocity and the degree of random, intrinsic scatter in polarization, and interpreted this finding as due to the presence of blobs that survive or grow more effectively in slow winds. Davies et al (2005) found this trend to continue into the regime of the LBVs, which have much lower $v_{\infty}$ than WR-winds and are an ideal testbed for constraining clumping properties, due to their long wind-flow times.

Davies et al (2005) performed a spectropolarimetric survey of LBVs in the Galaxy and the Magellanic Clouds and found more than 50\% of them to be intrinsically polarized. As the polarization angle was found to vary irregularly with time, the polarization line effects were attributed to wind clumping. Monte Carlo models for scattering off wind clumps were developed by Code and Whitney (1995), 


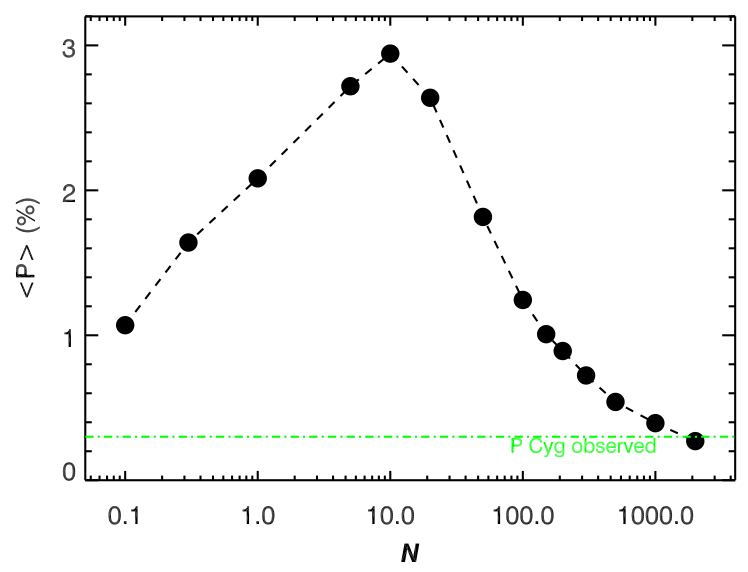

Fig. 26 Time-averaged polarization over a broad range of ejection rates per wind flow-time, $\mathscr{N}$. At $\mathscr{N} \sim 20$, the optical depth per clump exceeds unity and the overall polarization falls off (see Davies et al 2007 for details). The observed level of polarization for P Cygni is marked by the dash-dotted line. Note that there are two ejection-rate regimes where the observed polarization level can be achieved.

Rodrigues and Magalhaes (2000), Harries (2000), whilst analytical models to produce the variability of the linear polarization were presented by Davies et al (2007).

An example of an analytical model that predicts the time-averaged polarization for the LBV P Cygni is presented in Fig. 26, The clump ejection rate per wind flow-time $\mathscr{N}$ is defined as $\mathscr{N}=\dot{N} t_{\mathrm{fl}}=\dot{N} R_{\star} / v_{\infty}$, where the clump ejection rate, $\dot{N}$, is related to the mass-loss rate via $\dot{M}=\dot{N} N_{e} \mu_{e} m_{H}$, with $N_{e}$ the number of electrons in each clump and $\mu_{e}$ the mean mass per electron. There are two ejectionrate regimes where the observed polarization level can be achieved. One regime is where the ejection rate is low and a few optically thick clumps are expelled, and the other regime is that of optically thin clumps where the number of clumps is very large. These two models can be distinguished via time resolved polarimetry. Given the relatively short timescale of the observed polarization variability, Davies et al 2007 favor the latter scenario, suggesting that LBV winds consist of thousands of optically thin clumps in close proximity to the photosphere.

Stratification of the clumping factor. Most of the studies considered so far assume that clumping starts just above the sonic point and remains constant throughout the wind. We now address whether this approximation is feasible or needs to be relaxed by requiring stratified clumping. Obviously, this can be achieved by identifying reliable clumping diagnostics at different distances from the star. First indications that $f_{\mathrm{vol}}$ (or $f_{\mathrm{cl}}$ ) vary as a function of radial distance from the star were obtained by Nugis et al (1998) by means of IR to $\mathrm{mm}$ and radio continuum studies of WRs. To explain a spectral index from $\mathrm{mm}$ to $\mathrm{cm}$ wavelengths of the order of $\sim 0.76$ (steeper than a value of 0.6 expected for smooth winds, see Eq. 46), Nugis et al (1998) proposed a stratification of the clumping factor such that $f_{\mathrm{cl}}$ is unity at the wind base, reaches a maximum at 5-10 $R_{*}$, and then recovers unity in the outer wind parts. With respect to mass-loss rates derived from homogeneous 


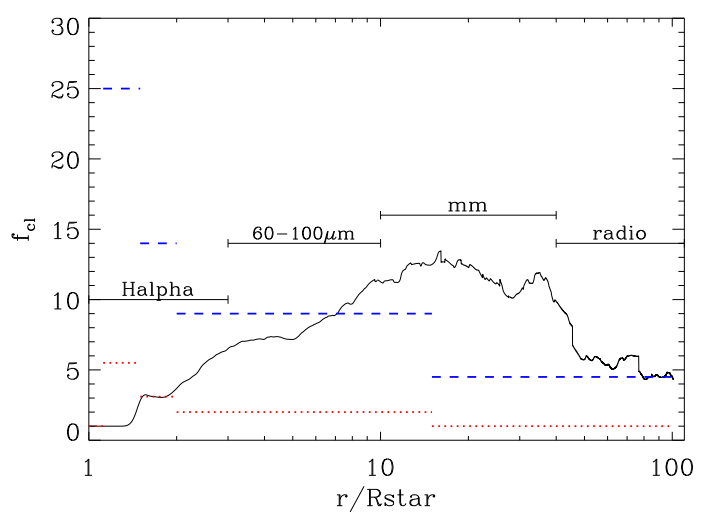

Fig. 27 Radial stratification of the clumping factor $f_{\mathrm{cl}}$ for an O-supergiant wind. Solid: Theoretical predictions by Runacres and Owocki (2002) from hydrodynamical models, with self-excited line-driven instability. The formation regions of different diagnostics are indicated. Dotted: Average clumping factors, as derived for the wind from $\zeta$ Pup, assuming an unclumped outer wind (Puls et al 2006). Dashed: as dotted, but assuming an outer wind with clumping as theoretically predicted. The corresponding mass-loss rate is a factor of $\left(f_{\mathrm{cl}}^{\text {out }}\right)^{0.5}$ smaller than in the dotted case. See text.

models, Nugis et al (1998) concluded that these were overestimated by $\sim 0.2 \mathrm{dex}$ for WN stars and by $\sim 0.6$ dex for WC star 38

A similar parameterization of the clumping factor was used by Figer et al (2002) in their analysis of WNL and $\mathrm{OIf}^{+}$stars in the Arches Cluster. Only a vanishing clumping $\left(f_{\mathrm{cl}}=1\right)$ in the outer wind zones of these objects could account for the observed $\mathrm{Pa}_{\alpha}$ equivalent widths and radio fluxes of the stars. These authors used $\mathrm{Pa}_{\alpha}, \mathrm{Br}_{\gamma}$, and the radio fluxes as diagnostics for the radial dependence of $f_{\mathrm{cl}}$.

A further step towards the characterization of wind clumping was taken by Puls et al (2006), who were able to derive constraints on the radial stratification of $f_{\text {cl }}$ by simultaneously modeling $\mathrm{H}_{\alpha}$ and the $\mathrm{IR} / \mathrm{mm} /$ radio emission from a sample of 19 O-stars with well-known parameters. Actual constraints could be obtained in the $\left(1-5 R_{*}\right)$ lower and intermediate wind region where $\mathrm{H}_{\alpha}$ and the IR form, and in the outer $\left(10-50 R_{*}\right)$ wind region where the $\mathrm{mm} /$ radio emission arises. Particularly for the best constrained object, $\zeta$ Pup, the derived stratification is in strong contrast to theoretical predictions (see Fig. 277). Actually, Puls et al (2006) found considerable clumping already close to the stellar surface for all denser winds, consistent with the findings from other diagnostics covered in previous sections. Clumping would then remain rather constant over a large volume before decreasing in the outer wind, with ratios of clumping factors between the inner and outer regions ranging from 3 to $6\left(\dot{M}\left(\mathrm{H}_{\alpha}\right)=1.7-2.5 \dot{M}_{\text {radio }}\right)$, which is very similar to the values suggested by Markova et al (2004) and Repolust et al (2004) on a completely different basis (page 73). For weaker winds, on the other hand, Puls et al (2006) found similar clumping factors for the inner and outermost regions, i.e., $\dot{M}\left(\mathrm{H}_{\alpha}\right) \approx \dot{M}_{\text {radio }}$.

\footnotetext{
38 In a subsequent study, Nugis and Lamers (2000) derived an empirical mass loss formula for
} Galactic WR stars accounting for these reduced rates, see Sect.4.2.3 


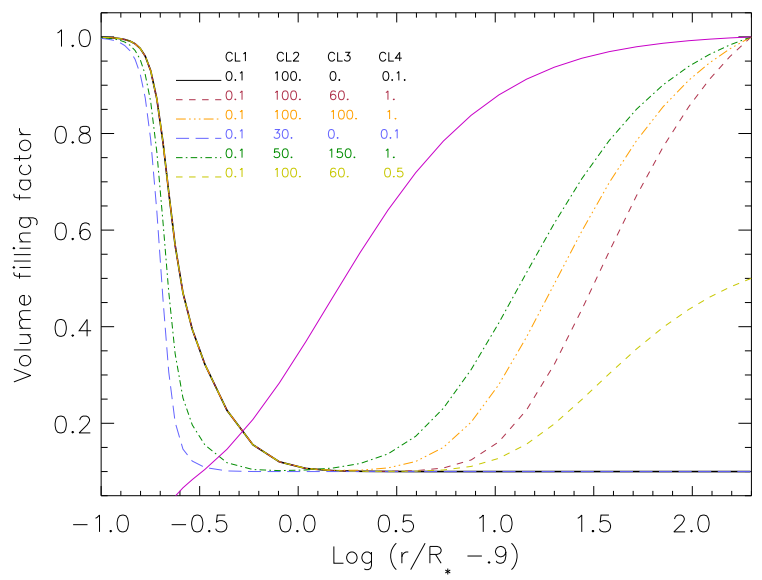

Fig. 28 Examples for the radial structure of the volume filling factor, $f_{\text {vol }}(r)$, for different values of $\mathrm{CL}_{2,3,4}$ according to Eq. 55 and $\mathrm{CL}_{1}=0.1$. For models with an unclumped outer wind, $\mathrm{CL}_{4}=$ 1 , whilst for models with constant clumping in the outer part, $\mathrm{CL}_{4}=\mathrm{CL}_{1}$. The velocity structure (solid magenta, in units of $v_{\infty}$ ) is displayed to illustrate the onset of the clumping variations.

A major shortcoming of this study is caused by the $\rho^{2}$ dependence of all considered diagnostics, which lead the authors to derive only relative clumping factors. Note, however, that mass-loss rates based on the assumption that $f_{\mathrm{cl}}$ (radio) $=1$, i.e., an unclumped outer wind, would give rise to a unique WLR (dependent only on $L$ ), being in very good agreement with the theoretical predictions by Vink et al (2000).

The next natural step is then to perform multi-wavelength analyses including both $\rho$ and $\rho^{2}$ diagnostics. These have been recently presented by Najarro (2008) and Najarro et al (2008) by means of UV to radio observations of OB stars and IR observations of LBVs. These authors identify key diagnostics lines to obtain the clumping structure throughout the stellar wind, and provide guidelines to constrain the degree of clumping in stellar winds for different stellar types.

To investigate the clumping stratification in detail, Najarro et al (2008) utilized the $\mathrm{O} \mathrm{If}^{+}$star CyOB2\#7 as a work bench and suggested the following clumping law (see Fig. 28):

$$
f_{\text {vol }}(r)=C L_{1}+\left(1-C L_{1}\right) \mathrm{e}^{\frac{-v(r)}{C L_{2}}}+\left(C L_{4}-C L_{1}\right) \mathrm{e}^{\frac{-\left(v_{\infty}-v(r)\right)}{C L_{3}}},
$$

where $\mathrm{CL}_{1}$ and $\mathrm{CL}_{4}$ are volume filling factors and $\mathrm{CL}_{2}$ and $\mathrm{CL}_{3}$ are velocity terms defining locations in the stellar wind where the clumping stratification changes. $\mathrm{CL}_{1}$ sets the maximum degree of clumping reached in the stellar wind (provided $\mathrm{CL}_{4}>\mathrm{CL}_{1}$ ) whilst $\mathrm{CL}_{2}$ determines the velocity of the onset of clumping. $\mathrm{CL}_{3}$ and $\mathrm{CL}_{4}$ control the clumping structure in the outer wind. This is illustrated in Fig. 28 , which displays the behavior of clumping in a stellar wind for different values of $\mathrm{CL}_{2}, \mathrm{CL}_{3}$ and $\mathrm{CL}_{4}$. The clumping law Eq. 55 has been constructed in such a way that as the wind velocity approaches $v_{\infty}$, the volume filling factor starts to change from $\mathrm{CL}_{1}$ towards $\mathrm{CL}_{4}$. If $\mathrm{CL}_{4}$ is set to unity, the wind will be unclumped in 


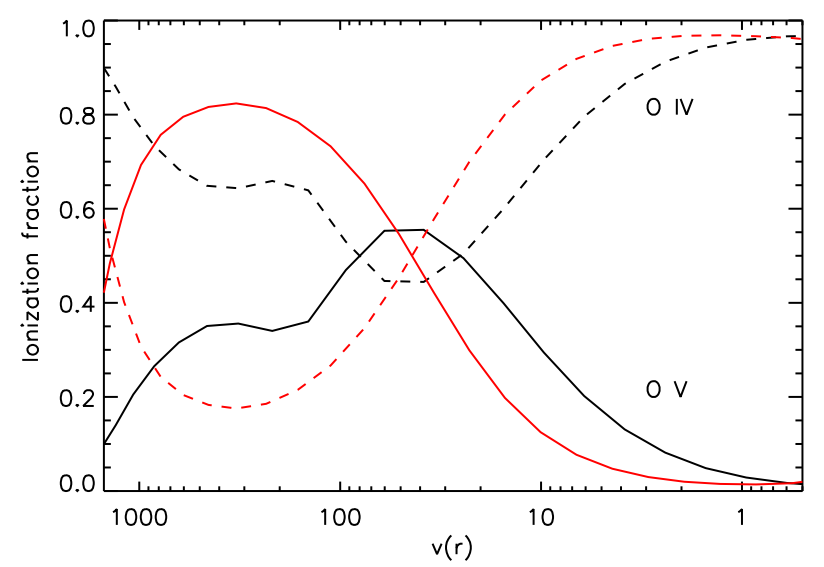

Fig. 29 Run of the O IV (dashed) and OV (solid) ionization fractions of an O supergiant wind for a clumped (black) and homogeneous (light color) model. Adapted from Bouret et al (2005).

the outermost region (as suggested by Nugis et al 1998 and adopted by Figer et al 2002 and Najarro et al 2004 for the case of WR-winds, see above). From Eq. 55 we note that for $\mathrm{CL}_{3} \rightarrow 0$ we recover the law as proposed by Hillier and Miller (1999).

All clumping effects considered so far refer to a situation where the ionization fraction of the ion responsible for the considered process is not or only weakly affected by the clumping itself (at least beyond the conventional $\dot{M} \sim \sqrt{f_{\text {vol }} \text {-scaling }}$ Eq. 54). Thus, for a resonance line (e.g., P v) it must be secured that the ionization stage the line belongs to $\left(\mathrm{P}^{+4}\right)$ clearly remains as the dominant stage, so that by varying the clumping alone no changes in the line profile are produced. On the other hand, for a recombination line, the ionization stage the line belongs to must be clearly less populated than the next higher ionization stage (i.e., $\mathrm{H} \mathrm{II} \gg \mathrm{H} \mathrm{I}$ for the hydrogen lines), so that an $\dot{M} \sim \sqrt{f_{\text {vol }}}$-scaling preserves the number of recombinations. We consider the corresponding regime of the ionization equilibrium to be on the "safe" side.

Most worrying is the case where two adjacent ionization stages have similar populations. Then clumping will cause a net reduction of the mean ionization, due to an enhanced number of recombinations $\left(\propto \rho^{2}\right)$ compared to ionizations (roughly $\propto \rho$ ). This will result in a line-profile behaviour similar to a reduction of the stellar temperature. We denote such a regime of the ionization equilibrium by the term "changing". This indirect effect of clumping has been warned about from early times on (e.g., Puls et al 1993b), and Bouret et al (2005) have investigated this effect in detail. As an example, Fig. 29 shows how the oxygen ionization equilibrium is significantly altered due to wind clumping.

One may, therefore, distinguish between lines formed in a "safe" region and those arising from a "changing" region. Figure 30 shows that the N IV $\lambda 4058$ and $\mathrm{N} v \lambda 4600$ lines in CygOB2 \#7 are clearly formed in such a "changing" region, whilst the rest of the optical lines are less affected by the large change of clumping and seem to follow the "safe" region behaviour. On the other hand, most of the infrared lines react strongly to changes in the clumping law. Since, as out- 

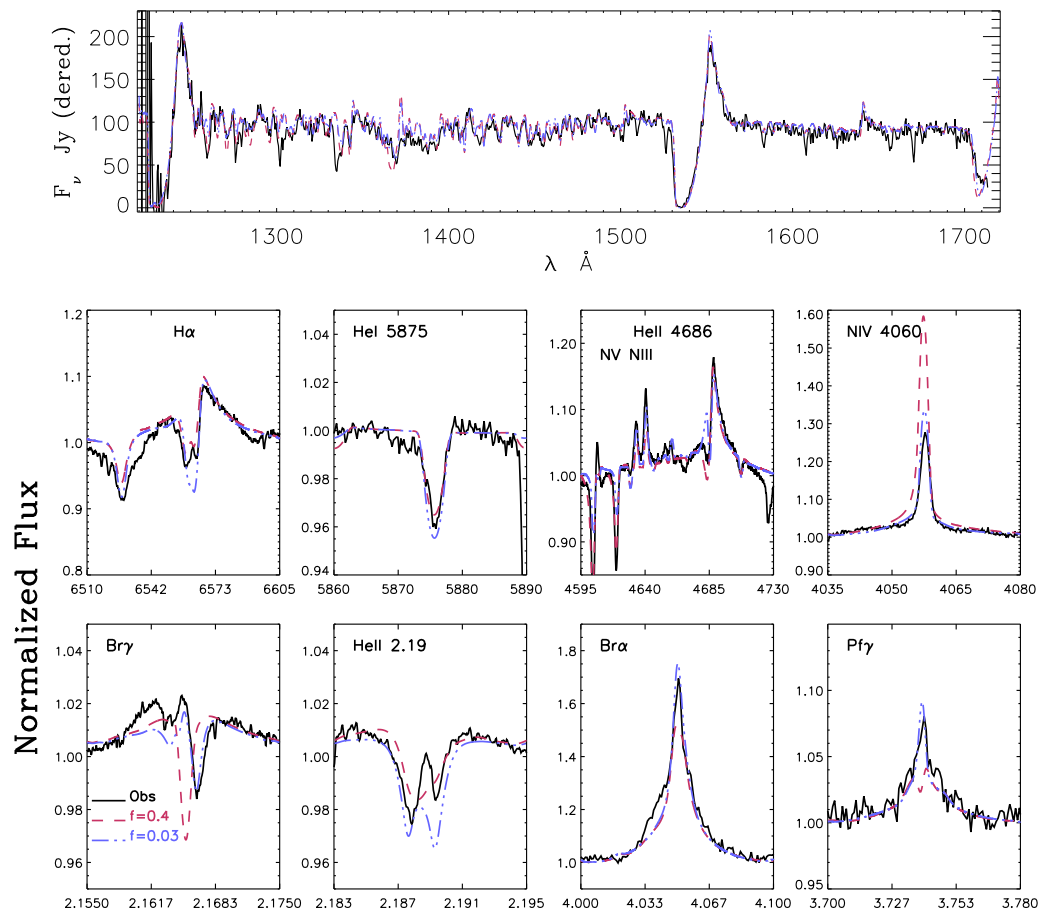

Wavelength

Fig. 30 Best model fits to UV, optical, and infrared profiles of CygOB2 \#7, for two values of $\mathrm{CL}_{1}$, denoted in the figure by $f$ and corresponding to the maximum degree of clumping in the wind (= minimum volume filling factor). The weakly clumped model (dashed) with $\mathrm{CL}_{1,2,3,4}=$ $\left(0.4,350 \mathrm{~km} \mathrm{~s}^{-1}, 25 \mathrm{~km} \mathrm{~s}^{-1}, 1\right.$.) has a mass-loss rate of $6 \cdot 10^{-6} M_{\odot} \mathrm{yr}^{-1}$, whereas the strongly clumped model (dashed-dotted) with a clumping law according to Hillier and Miller (1999), $\mathrm{CL}_{1,2}=\left(0.025,120 \mathrm{~km} \mathrm{~s}^{-1}\right)$, has $\dot{M}=1.4 \cdot 10^{-6} M_{\odot} \mathrm{yr}^{-1}$.

lined on page 42, the continuum adjacent to NIR-lines is already formed in the wind, not only the lines, but also the continuum will depend on clumping (via bound-free and free-free processes $\propto \rho^{2}$ ), resulting in a high sensitivity of the continuum-rectified line profiles on the clumping stratification, particularly on $\mathrm{CL}_{1}$ and $\mathrm{CL}_{2}$. Najarro et al (2008) found that if clumping starts with large clumping factors relatively close to the photosphere of $\mathrm{CygOB} 2 \# 7$ (with $f_{\mathrm{cl}}=5$ reached at $\sim 200 \mathrm{~km} \mathrm{~s}^{-1}$, corresponding to $\sim 1.1 R_{*}$, similar to the results by Puls et al 2006 for this object), consistent simultaneous fits to the UV, optical, and IR observations are possible (see Fig. 30).

Whilst the optical and IR spectra of CygOB2 \#7 provide strong constraints on $\mathrm{CL}_{1}$ and $\mathrm{CL}_{2}$, the $\mathrm{UV}$, submillimeter, and radio observations constitute crucial diagnostics to determine $\mathrm{CL}_{1}$ and $\mathrm{CL}_{3}$. Indeed, $\mathrm{UV}$ and submillimeter data support the presence of constant clumping (Najarro et al 2008), at least up to mid/outer wind regions where the millimeter continua of $\mathrm{CygOB} 2$ \#7 are formed. However, radio observations by Puls et al (2006) with a formation region at much larger radii 
showed that clumping may begin to vanish in the outermost wind regions, which is consistent with the fact that the radio emission from such models with constant clumping severely overestimates the upper limits provided by the observations by Puls et al (2006) of CygOB2 \#7. This demonstrates the need of multi-wavelength observations to constrain the run of the complete clumping stratification.

Finally, for quantitative analyses one would wish to have a nice toolkit providing diagnostic lines that, depending on the stellar type of the object, could be used to constrain the absolute degree of wind clumping. At temperatures around $30 \mathrm{kK}$, there is the possibility to use $\mathrm{He}$ II 4686 and $\mathrm{H}_{\alpha}$ in parallel, as outlined on page 51. Otherwise, one has particularly to rely on the "changing" region situation discussed above. No strong UV lines should be included as they may be significantly affected by X-rays and corresponding EUV emission. Note, however, that even UV-lines formed in the lower wind could be affected by very hot material potentially located close to the wind base (see Sect.6.5). For early O supergiants, the $\mathrm{N}$ IV $\lambda 4058$ and $\mathrm{N} v \lambda 4600$ lines, and for late O supergiants, the He II, N III and $\mathrm{C}$ III lines, turn into important clumping diagnostic lines. Valuable clumping information may be obtained from cool LBVs for which the He I and Fe II] (Najarro 2008) lines will arise from "changing" regions.

\subsubsection{Treatment of clumping - Micro- and macro-clumping}

With studies yielding clumping factors ranging from $f_{\mathrm{cl}}=2$ to $\gtrsim 100$ (corresponding to volume filling factors $f_{\mathrm{vol}}=0.5$ to $\lesssim 0.01$ ), one may question whether our present treatment of clumping is physically correct. If related to a certain type of instability (e.g., the line-driven one), we may expect this treatment to be inadequate in those regions where the instability is not fully grown but still in its linear or only weakly non-linear phase. In these (lowermost) wind regions, the assumption of a void inter-clump medium is certainly questionable. Most of the atmospheric codes take into account only variations in the density, but the hydrodynamic simulations also reveal strong changes of the velocity field inside the clumps, sometimes even changing the sign of the velocity gradient. Most important, however, is the recent finding that the prerequisite of the standard microclumping approach, namely that the clumps are optically thin, needs to be relaxed.

Within the micro-clumping approach (page 74), an optically thin clump is identified as a clump with a size smaller than the photon mean free path of the relevant matter-light interaction. In an optically thick clump ("macro-clumping"), photons will interact with matter many times before eventually being destroyed or scattered off the clump and escaping through the inter-clump matter. Of course, whether a clump is optically thin or thick will depend on the abundance, ionization fraction, and cross-section of the involved transition. Thus, we will have clumps that are optically thick for some processes while being optically thin for others. For a recent review of micro- vs. macro-clumping, we refer the reader to Hamann et al (2008a, see also Oskinova et al 2007).

Micro-clumping has been firstly implemented into NLTE model atmospheres by Schmutz (1995), and the corresponding formalism was outlined on page 74. For our discussion of macro-clumping we follow the same notation (see also Owocki et al 2004 and the review by Hamann et al 2008a). 
For optically thick clumps, photons $d o$ care about the distribution, the size and the geometry of the clump (see Sect. 6.5) they will encounter and multi-interact with. The conventional description of macro-clumping bases on a uniform clump size, $l(r)$, and an average separation among the statistically distributed clumps, $L(r)$, which are related by the volume filling factor,

$$
f_{\mathrm{vol}}=\left(\frac{l}{L}\right)^{3}=\frac{1}{f_{\mathrm{cl}}} \text {. }
$$

Following the nomenclature of Eq. 53, the optical depth across a clump of size $l$ and constant opacity $\kappa_{C}$ is given by

$$
\tau_{C}=\kappa_{C} l=\bar{\kappa} f_{\mathrm{cl}} l=\bar{\kappa} \frac{L^{3}}{l^{2}}=\bar{\kappa} h,
$$

with mean opacity $\bar{\kappa}$ (Eq. 53) and porosity length $h=L^{3} / l^{2}$, as introduced in Eq.47 Remember that the porosity length constitutes a key parameter to quantify the characteristics of a structured medium and corresponds to the photon mean free path in a medium consisting of optically thick clumps (page 56).

The effective cross section of the clumps, i.e., their geometrical cross section (surface) diminished by the fraction of transmitted radiation, is given by

$$
\sigma_{C}=l^{2}\left(1-\mathrm{e}^{-\tau_{C}}\right)
$$

such that the effective opacity that needs to be used in the models results in

$$
\kappa_{\text {eff }}=n_{C} \sigma_{C}=\frac{l^{2}\left(1-\mathrm{e}^{-\tau_{C}}\right)}{L^{3}}=\frac{\left(1-\mathrm{e}^{-\tau_{C}}\right)}{h}=\bar{\kappa} \frac{\left(1-\mathrm{e}^{-\tau_{C}}\right)}{\tau_{C}},
$$

when $n_{C}$ is the number density of the clumps. Note that this equation holds for clumps of arbitrary optical thickness. When taking the optically thin limit, we immediately recover the micro-clumping approximation, $\kappa_{\text {eff }}=\bar{\kappa}$, which depends on the volume filling factor alone but not on clump sizes and distribution, whereas in the optically thick case, the effective opacity becomes strongly reduced, $\kappa_{\mathrm{eff}}=$ $\bar{\kappa} / \tau_{C}=h^{-1}$ and depends only on the porosity length, i.e., it is grey. Further comments on effects resulting from specific clump geometries are given in Sect. 6.5.

Effects on wind structure. Krtička et al (2008) have studied the influence of micro- and macro-clumping on the predicted wind structure of O-type stars, by artificially including clumping into their stationary wind models which have been described in Sect. 3.1 .

If they assumed all clumps to be optically thin, the radiative line force increased compared to corresponding unclumped models, due to an increase of the electron density dependent $\delta$-term in the force-multiplier (page 9), representing a changed ionization equilibrium with more lines from lower stages. This increase of the line-force leads to an increase of either the mass-loss rate or the terminal velocity, depending on the onset of wind-clumping (below or above the critical point). Similar results have been found by de Koter et al (2008).

If, on the other hand, the clumps were considered as optically thick, Krtička et al (2008) showed that a net reduction of the line-force may occur. Note, however, that this finding is in contrast to hydrodynamical simulation $\$$, and originates from as-

\footnotetext{
39 which do not find changes of the gross wind properties compared to a stationary solution, though the medium is clumpy (page 71).
} 

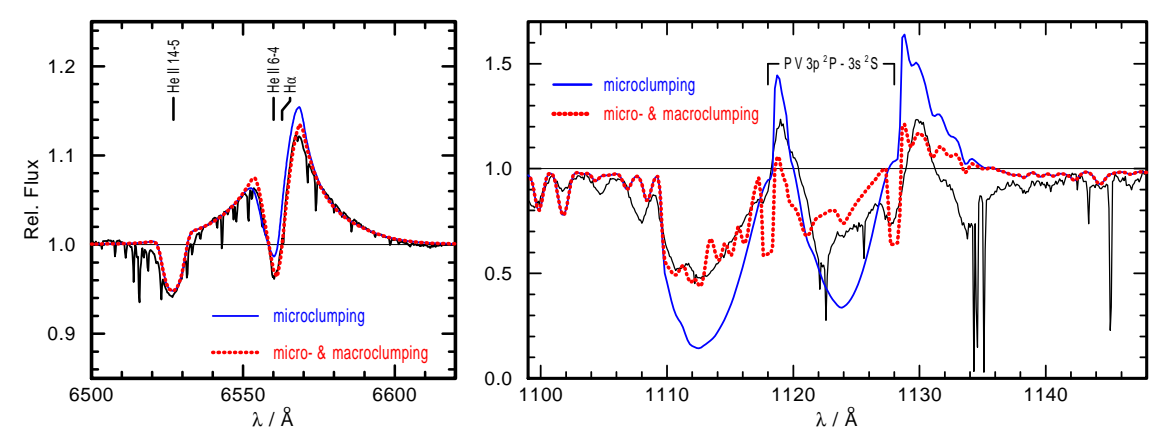

Fig. 31 Porosity as a possible solution for the PV problem. Adapted from Oskinova et al (2007), see text.

suming that the velocity law remains unchanged, in contrast to what would happen in a consistent approach. Finally, an inclusion of wind porosity into the continuum opacity (with the onset of porosity below the critical point) might also lead to a reduced $\dot{M}$, due to an increase of the wind-ionization.

In conclusion, a further, consistent investigation of the impact of wind-clumping (with its various facets) on the wind structure of massive $\mathrm{O}$ stars is crucial, since this might influence the predicted wind parameters, to which the observed ones have to be compared.

Effects on line profiles. Oskinova et al (2007) investigated the effects of porosity on the line profiles of $\zeta$ Pup, by means of the above formalism (Eq. 59) and assuming a "high" value for the mass-loss rate, $\dot{M}=2.510^{-6} M_{\odot} \mathrm{yr}^{-1}$, which is only mildly lower than the upper limit derived by Puls et al (2006). Using overdensities, $f_{\mathrm{cl}}$, inferred from diagnostic lines, and the equation of continuity to parameterize the radial dependence of the clump separation, $L(r)$, they were able to account for (part of 0 ) the radiative transfer effects of clumping.

In particular, only changes in the "formal integral" were considered (by using the effective opacity), whereas the feedback of macro-clumping on the NLTEpopulations was neglected by arguing that most transition rates are not affected. From Fig. 31 it becomes clear that the most important effect regards strong resonance lines like $\mathrm{P} \vee \lambda \lambda 1118-1128$, which can be reproduced by this approach without relying on (very) low mass-loss rates, due to the reduction of the effective opacity when the clumps become optically thick. On the other hand, $\mathrm{H}_{\alpha}$ is not affected by wind porosity, remaining optically thin inside the clumps and being reproduced in parallel with $\mathrm{P} v$. Insofar, these results might open a way out to the "P v problem".

Clumping from the other side: "Vorosity". As indicated above, line transitions should be strongly affected by a strongly non-monotonic velocity field as resulting from the line-driven instability. Aiming to examine the potential role of such a

\footnotetext{
40 Note that the original porosity concept had been developed for continuum processes; line processes are additionally affected by changes in the velocity field, which are simulated here by adopting a velocity dispersion inside the clumps (cf. page 36), but see below.
} 

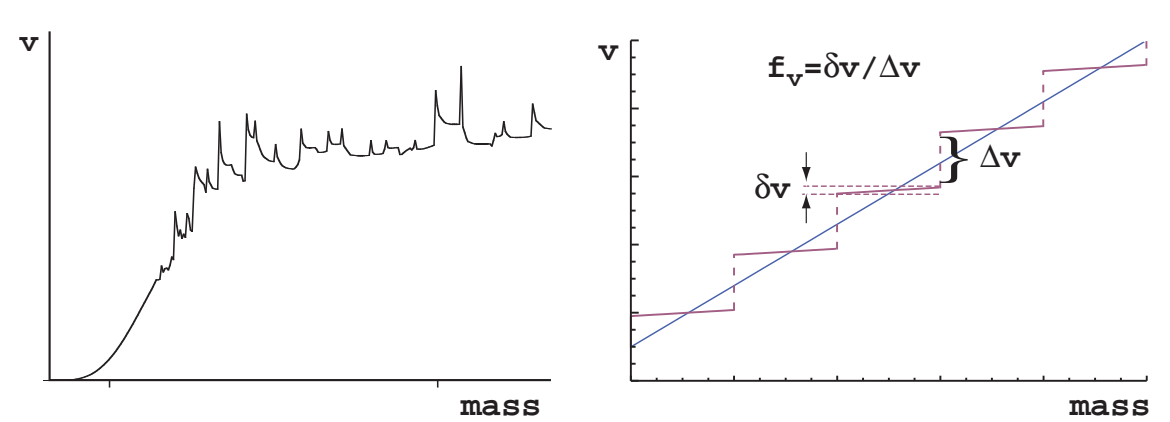

Fig. 32 Vorosity. Multiply non-monotonic velocity structure arising in a 1-D hydrodynamic simulation with self-excited line-driven instability, plotted versus a mass-coordinate (left), and schematic simplification (right). The smooth wind flow is represented by the straight line. Adapted from Owocki (2008), see text.

flow structure in reducing the observed strength of wind absorption lines, Owocki (2008) performed dynamical simulations of the line-driven instability in which the line strength can be described in terms of a "velocity clumping" factor. Interestingly, this velocity clumping factor is insensitive to spatial scales.

The left panel of Fig. 32 shows the velocity structure arising in a 1-D simulation. The intrinsic instability of line-driving leads to substantial velocity structure, with narrow peaks corresponding to spatially extended but tenuous regions of high-speed flow. Such regions are followed by dense, spatially narrow clumps/ shells which, when plotted as a function of mass-coordinate, give rise to extended velocity plateaus. The right panel illustrates the situation by means of a simplified model, where the velocity clumping is represented by a simple staircase structure, quantified by a velocity clumping factor. This value is set by the ratio between the internal velocity width $\delta v$ to the velocity separation $\Delta v$ of the clumps.

Using this model, Owocki (2008) computed dynamic spectra directly from instability simulations, which indeed exhibited a net reduction in absorption, due to the porosity in velocity space, an effect he denoted by "vorosity". The key problem in explaining a "reduced" $\mathrm{P} \vee$ line-strength in terms of vorosity is that one needs to have a large number of big velocity gaps, which is not what comes "naturally" from the instability simulations (Fig. 32- left). Thus, only a modest reduction at a 10-20\% level was found, which is well short of the factor 10 (or more) required in terms of the $\mathrm{P} v$ problem.

Obviously, it remains a future challenge to investigate scenarios including both porosity and vorosity and their possible inter-relation.

\subsubsection{Evolutionary limits}

If mass-loss rates would need to be revised drastically (e.g., by factors of $\sim 10$ or more), this would have dramatic consequences for the evolution of and feedback from massive stars. Recalling a corollary statement in massive star evolution, that a change of the mass-loss rates of massive stars by even a factor of two has a dramatic effect on their evolution (Meynet et al 1994), one immediately realizes the importance of quantifying the wind clumping phenomenon. This statement 
illuminates a potential alternative to constrain the "allowed" range of mass-loss reductions, namely by evolutionary considerations themselves.

Hirschi (2008) has recently explored the evolutionary implications. Taking for example a $120 M_{\odot}$ star with a lifetime of 2.5 million years and an average $\dot{M} \sim$ $2.5 \cdot 10^{-5} M_{\odot} \mathrm{yr}^{-1}$, the star will have lost around $50 M_{\odot}$ at the end of the main sequence using the theoretical prescriptions of Vink et al 2000, 2001, whilst the star would lose only $5 M_{\odot}$ if the mass-loss rate was reduced by a factor of 10 . The two big questions regarding evolution are then how to produce WR-stars with such low mass-loss rates and whether one would end up with too many critically rotating stars.

Concerning the WR issue, one way out would be to shift the bulk of mass loss on other evolutionary phases such as the LBV (e.g. Nugis and Lamers 2000, Smith and Owocki 2006) and the red supergiant (RSG) stages. However, although mass-loss rates are more challenging to determine in these two stages, it seems unlikely that they could cope with the bulk of it.

A second, provocative, possibility is to place all massive stars in close binary systems (Kobulnicky and Fryer 2007). This scenario, however, would not only fail to produce the many RSG stars observed, but there is also observational evidence against all WR-stars being in close binary systems (only 30-40\% in the Magellanic Clouds, Foellmi et al 2003a,b). Another possibility is to assume that WRs are produced by strong rotational mixing rather than by mass loss. Since fast rotators constitute only a small fraction of the whole population, this scenario seems very unlikely though.

Combining the above points, Hirschi (2008) concluded that evolutionary models could survive with $\dot{M}$ reductions of at most a factor of $\sim 2$ in comparison to the rates from Vink et al (2000), whilst factors around 10 are strongly disfavored. We note that a reduction of the Vink et al $(2000,2001)$ rates by a factor of two may correspond to an "allowed" reduction of the empirical mass-loss rates of a factor of about four - at most.

\subsection{Co-rotating interaction regions (CIRs)}

In the previous section, we have extensively discussed features attributed to microstructure in the wind, expressed in terms of micro- and macro-clumping, and consequences of this. However, there is also large-scale structure present, inferred most directly from the so-called discrete absorption components (DACs).

Discrete absorption components. These are optical depth enhancements observed in the absorption troughs of unsaturated UV P Cygni profiles and are present in most O-/early B-star winds (Howarth and Prinja 1989), but also in late B-supergiants (Bates and Gilheany 1990) and at least one WN7 star (Prinja and Smith 199241.

Typically, these DACs accelerate to the blue wing of the profile on timescales of a few days, becoming narrower as they approach an asymptotic velocity. Howarth and Prinja (1989) and Prinja et al (1990) have used this asymptotic value to determine $v_{\infty}$ for

41 Absorption components have been also observed in the Balmer lines of the LBV P Cygni (Markova 1986). 
objects with no black trough in CIV (see Sect. 4.1). The acceleration and recurrence time scales of DACs were found to be correlated with $v \sin i$, i.e., with the rotational period (Prinia 1988, Henrichs et al 1988, Kaper et al 1999). The acceleration of most strong DACs is much slower than the mean wind acceleration (Prinja et al 1992, Prinja 1994), suggesting that DACs might arise from a slowly evolving perturbation through which the wind material flows. Such a perturbation could consist of a higher density or a lower velocity gradient (e.g., a velocity plateau, as speculated already by Lamers et al 1982), or result from a combination of both (Fullerton and Owocki 1992).

Though the lack of variability in the emission component of P Cygni lines (Prinja 1992) and the lack of significant infrared variability (Howarth 1992) seems to rule out a spherically-symmetric disturbance, the structure must be large enough to cover a substantial fraction of the stellar disk in order to produce the observed strong absorption features. The latter constraint strongly argues against any microstructure related to the wind instability as the source of the DACs, because (i) micro-structure would be averaged out and (ii) the presence of the lateral linedrag (Rybicki et al 1990) should suppress any lateral velocity disturbances and maintain lateral length scales as set by a potential base perturbation.

Dynamical models of CIRs. The above arguments suggest that DACs should originate from coherent structures of significant lateral extent, with a clock related to stellar rotation and an increased optical line depth due to density and/or velocity field effects. Already in 1984, i.e., well before these constraints were known, Mullan had suggested co-rotating interaction region 42 (CIRs) as a potential candidate (see also Mullan 1986). Over the following years, this suggestion became more and more likely, due to an amount of empirical knowledge and since CIRs are compatible with all constraints as outlined above.

Cranmer and Owocki (1996) were the first to investigate the CIR scenario in detail, by means of 2-D time-dependent hydrodynamic modeling of the wind of a rotating O-star. Photospheric disturbances in the form of azimuthal variations were induced by a local increase of the line force 43 due to a bright stellar spot in the equatorial plane. Such a stellar spot should be regarded as a representative for various kinds of photospheric disturbances (e.g., localized magnetic fields or non-radial pulsations), since the induced wind structure turned out to be rather insensitive to the particular way by which the photospheric conditions were disturbed.

A locally enhanced line acceleration generates streams of higher density and lower velocity (compared to the undisturbed mean flow), and CIRs of enhanced density form where the faster mean flow collides supersonically with the slow material at the trailing edge of the stream. When the unperturbed wind collides with the CIR, a non-linear signal is sent back towards the star which forms a sharp, propagating discontinuity ("Abbott-kink", see also Feldmeier and Shlosman 2000, 2002) in the radial velocity gradient. The inwards directed propagation velocity (with respect to a comoving frame) of this kink is just the characteristic speed of

\footnotetext{
42 well known and studied in the solar wind.

43 Actually, the authors investigated also the consequences of a local decrease in the line force due to dark spots. Such a model, however, could be ruled out, since the resulting synthetic profiles behave quite different from the observed variability pattern.
} 
the radiative-acoustic Abbott waves considered in Sect.6.2 In the stellar frame, this feature travels slowly outwards and a velocity plateau-like structure is formed between the trailing Abbott-kink and the CIR compression.

By calculating the corresponding line optical depths and time-dependent ("dynamic") synthetic profiles, Cranmer and Owocki (1996) showed that these slowly moving kinks, together with their low velocity gradients $\left(\tau \propto(\mathrm{d} v / \mathrm{d} r)^{-1}\right.$ !), are the likely origin of the observed DACs rather than the CIRs themselves (but see below). A fit to the temporal wavelength drift of the synthesized DACs resulted in a velocity field parameter $\beta \approx 2-4$, i.e., in a slow apparent acceleration, in accordance with observations. Though the recurrence time scale of the DACs is strictly correlated with $v_{\text {rot }}$, due to the link of CIRs and the rotating photosphere, no correlation between acceleration time scale and rotational speed was found in the synthetic profiles, in contrast to the observational indications as outlined above.

This "missing" correlation is the central topic of an interesting kinematical investigation by Hamann et al (2001), who stressed the fact that the low drift rate is not a consequence of the CIRs themselves. Features formed within the CIR would display a drift even faster than features formed in a non-rotating wind. Instead, the slow drift is a consequence of the difference between mean flow and the velocity field of the pattern in which the features form. A pattern with upstream propagation (as the kink in the model by Cranmer and Owocki 1996) inevitably results in a wavelength drift with a slower apparent acceleration than displayed by features formed in the bulk or within the CIR itself. Any of these drifts, however, were shown to be independent on the rotation rate, leaving the observed correlation (if actually present) still unexplained.

An implicit assumption in the work by Hamann et al (2001) is that the CIRs are induced by disturbances locked to the stellar surface, in accordance with the model by Cranmer and Owocki (1996). In a detailed study, Lobel and Blomme (2008) relaxed this assumption in order to enable a quantitative analysis of the time evolution of DACs for the fast-rotating B0.5 Ib supergiant HD 64760, one of the best observed objects (see below). The authors use their own 3-D radiative transfer code, coupled with 3-D hydrodynamics restricted to the equatorial plane. A large grid of models and dynamic spectra for different spot parameters (brightness, opening angle and velocity) has been computed, and the effects of these parameters on the wind structure and DAC evolution studied. A best fit could be obtained with a model with two spots of unequal brightness and size on opposite sides of the equator, with spot velocities being five times slower than $v_{\text {rot }}$. All basic conclusions of Cranmer and Owocki (1996) could be confirmed, particularly the importance of velocity plateaus in between kinks and CIRs. For their models of spots which are not locked to the stellar surface, however, the independence of the DAC acceleration on $v_{\text {rot }}$ can no longer be warranted. In this case, the internal clock is given by the rotational speed of the spots, $v_{\mathrm{sp}}$, whereas a change in the stellar rotational speed modifies the underlying bulk flow (densities and velocities) via a different centrifugal acceleration (Eqs. 20, 21) such that the time-scales are no longer conserved when $v_{\text {rot }}$ is changed. In other words, two models with $v_{\mathrm{sp}}=v_{\text {rot }}$ (locked) and $v_{\mathrm{sp}}<v_{\text {rot }}$ (non-locked) which both reproduce the DAC recurrence time behave differently with respect to the acceleration time scale.

The physical scenario of different spot and stellar rotation velocities follows a suggestion by Kaufer et al (2006). These authors detected non radial pulsations 
in the photospheric lines of HD 64760, with closely spaced periods. In order to explain the observed $\mathrm{H}_{\alpha}$ variability, they invoked the beat period between two of these periods (lower than the rotational one!) to be responsible for the CIRs in the wind. Interestingly, however, this beat period is not consistent with the spot velocity derived from the UV by Lobel and Blomme (2008). For further discussion we refer to the latter publication.

Reality or artefact? As should be clear from our discussion of the onset of Abbott-waves, the dynamic models discussed above rely on a Sobolev line-force, because of computational feasibility. Thus, two questions need to be answered.

(i) In how far do the kinks still occur when the Sobolev approximation is relaxed? (ii) In how far can the line-driven instability disrupt the CIR structure? A preliminary answer might be found in Fig. 2c from Owocki (1999), which displays the radial velocity calculated for a CIR model based on a non-local line force calculated from a three-ray treatment (cf. page 70). Also here, somewhat "rounded" kinks followed by a plateau are present! Unfortunately, however, the line-driven instability effectively destroys all macro-structure, but this might be related to a still inadequate three-ray treatment and/or insufficient resolution, leading to too low a lateral damping. To cite Stan Owocki (2008, priv. comm.), "there is a strong potential that such kinks and plateaus are real physical effects in a line-driven wind, and not just an artefact of the Sobolev approximation."

Future work using computationally expensive methods to calculate a consistent more-D line force will hopefully prove this expectation.

Modulation features. The observed correlations of the DAC properties with $v \sin i$ inspired the "IUE MEGA Campaign" (Massa et al 1995), during which three prototypical massive stars ( $\zeta$ Pup (O4If), HD 64760 (B0.5Ib) and HD 50896 (WN5)) were monitored almost continuously over 16 days. Results of this campaign have been summarized by Fullerton (1999). The most important finding was the detection of a new type of variability, namely periodic modulations in the UV wind lines, derived from the dynamic spectra of HD 64760 (Prinia et al 1995) which most likely is observed equator-on. As shown by Fullerton et al (1997), these modulations result from two quasi-sinusoidal fluctuations with periods of 1.2 and 2.4 days, which propagate, as a function of phase and starting at roughly $750 \mathrm{~km} \mathrm{~s}^{-1}$, simultaneously towards lower and higher line-of-sight velocities, until a certain minimum and maximum is reached and the pattern begins to repeat itself. Because of its peculiar shape, this effect has been called "phase bowing". Similar features (though not as pronounced) have been detected in only two other stars, $\zeta$ Pup (Massa et al 1995, Howarth et al 1995) and $\xi$ Per (O7.5 III (n)((f)), Kaper et al 1999). Note that these features occur in parallel with the conventional DACs.

The peculiar phase properties of the modulation features indicate the presence of absorbing material with the same phase at two different projected velocities. By means of a kinematical model and corresponding synthetic spectra, Owocki et al (1995) and Fullerton et al (1997) suggested that these features are formed in azimuthally extended, co-rotating and spirally-shaped wind structures linked to a surface density which is modulated by non-radial pulsations. Due to rotation, a certain spiral exits from the lines of sight in front of the stellar disk first at an intermediate projected velocity $\left(750 \mathrm{~km} \mathrm{~s}^{-1}\right.$ in case of $\left.\mathrm{HD} 64760\right)$. At later times 
and because of its curvature, this structure exits from the absorption region simultaneously at lower and higher velocities. Since the spirals are assumed to be linked to the surface density modulation, different locations along the spiral have the same phase, because they arise from the same "spot" on the surface. In concert with the kinematics of the spirals, this explains the modulation features and their phase-bowing, verified in the aforementioned synthetic spectra. Consistent hydrosimulations of this scenario are still missing, but these features are a strong hint on the presence of azimuthally extended macro-structure and observational "evidence for co-rotating wind streams rooted in surface variations" (Owocki et al 1995).

\subsection{X-ray line emission}

After the advent of the X-ray satellites XMM-NEWTON and CHANDRA, with their capability to perform high-resolution spectroscopy, detailed knowledge about the $\mathrm{X}$-ray line emission from hot stars has been accumulated (for recent reviews, see Oskinova et al 2008 and Cohen 2008). Two "classes" of spectra have been found. On the one side, the prototypical spectrum of $\theta^{1}$ Ori $C$, one of few massive stars with a strong magnetic field (Sect. 2.2.2), is rather hard (implying high plasma temperatures) and shows lines with small widths. On the other side, the spectrum of the prototypical "normal" supergiant $\zeta$ Pup is softer and displays much broader, asymmetric lines. Moreover, the lines from hydrogen-like ions are stronger in the former class, and vice versa for the lines from helium-like ions, which immediately points to differences in the ionization balance and thus again to different plasma temperatures. Wojdowski and Schulz (2005) derive a peak value $T=30 \cdot 10^{6} \mathrm{~K}$ for $\theta^{1}$ Ori $\mathrm{C}$ and $1-2 \cdot 10^{6} \mathrm{~K}$ for six other O-stars (incl. $\zeta$ Pup), where the latter values are of the same order as those derived from earlier, low resolution data (e.g., by ROSAT, Kudritzki et al 1996, see Sect.6.1).

\subsubsection{Magnetically confined winds}

By means of a tailored MHD mode ${ }^{44}$ for the wind of $\theta^{1}$ Ori C, Gagné et al (2005) showed that the magnetically confined wind shock (MCWS) model (discussed in Sect.2.2.2 fits quite well all the data, predicting the temperature, luminosity, and occultation of the X-ray emitting plasma with rotation phase. Particularly, the bulk of the shock-heated plasma should be located in the magnetically confined region close to $r \approx 2 R_{*}$, where the speed and line-of-sight velocity of this material is low, giving rise to rather narrow X-ray emission lines. Similar arguments might hold for the X-ray spectrum of $\tau$ Sco which also shows narrow lines, consistent with the finding that also $\tau$ Sco has a strong magnetic field.

\subsubsection{Non-magnetic stars}

In accordance with previous results derived from lower resolution observations (Sect. 6.1), also the gross properties of highly resolved X-ray spectra from "normal" O-stars may be well explained by the line-driven instability scenario (either

\footnotetext{
44 extending the work by ud-Doula and Owocki (2002) with respect to the energy equation.
} 


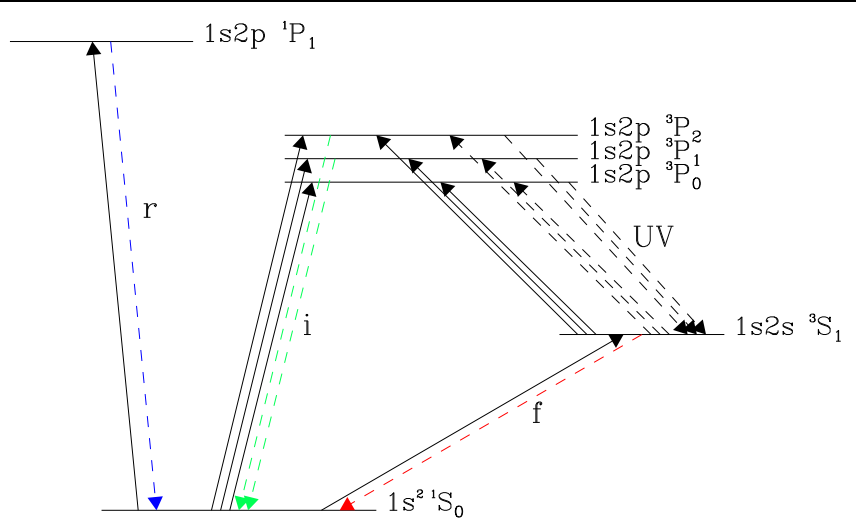

Fig. 33 Schematic Grotrian diagram for Helium-like ions, showing levels and transitions involved in the formation of the fir-complex in X-ray spectra of hot stars. Solid arrows: collisional excitations. Dashed arrows: radiative transitions. The f,i,r transitions are denoted by corresponding letters. The transitions between the ${ }^{3} \mathrm{~S}$ and the ${ }^{3} \mathrm{P}$ levels are located in the (F)UV. Adapted from Gabriel and Jordan (1969).

self-excited or triggered, with dominating clump-clump collisions). This refers particularly to the softness of the spectra and the large line widths, resulting from the high velocity of the shock-heated wind. Remember, e.g., that the line-driven instability should be fully grown above 1.3-1.5 $R_{*}$ and that the predicted shock temperatures are of the order of "only" a few million Kelvin, due to jump velocities of a few hundred $\mathrm{km} \mathrm{s}^{-1}$.

fir-diagnostics of Helium-like ions. A key diagnostics to observationally constrain the onset of the X-ray emission is provided by the ratio of line fluxes from Helium-like ions such as OVII, NeIX, MgXI and SiXIII. These ions show characteristic fir triplets (Gabriel and Jordan 1969) of a forbidden (f), an intercombination (i) and a resonance (r) component (cf. Fig. 33).

In hot stars, the $\mathscr{R} \equiv f / i$ line ratio is sensitive to the local mean intensity of the UV radiative field, since UV photons can excite the metastable ${ }^{3} \mathrm{~S}_{1}$ level to the ${ }^{3} \mathrm{P}_{0,1,2}$ levels. To a good approximation, this mean intensity is given by $W(r) H_{v}^{\text {phot }}$, with dilution factor $W(r)$ and photospheric flux $H_{v}^{\text {phot }}$. If the latter is known (from observation 45 or model atmospheres), the dilution factor $W(r)$ may be inferred from $\mathscr{R}$, which constrains the formation radius $R_{\text {fir }}$ of the X-ray emitting plasma. Applying this method, Leutenegger et al (2006) ${ }^{46}$ studied the $X$ ray lines of Helium-like ions in four evolved O-stars (incl. $\zeta$ Pup) and derived minimum radii of X-ray line formation in the range of $1.25 R_{*}<R_{\mathrm{fir}}<1.67 R_{*}$, consistent with the theoretical expectation.

On the other hand, Waldron and Cassinelli (2007) published rather puzzling results for a larger sample of OB-stars. Based on similar fir diagnostics, they concluded that high-Z ions were predominantly located closer to the photosphere, a

\footnotetext{
45 if the transition is located in the observable part of the UV spectrum, and not contaminated by wind-lines.

46 see also Leutenegger et al (2007) for the effects of resonance scattering in the r-component, which give rise to more symmetric fir-complexes.
} 


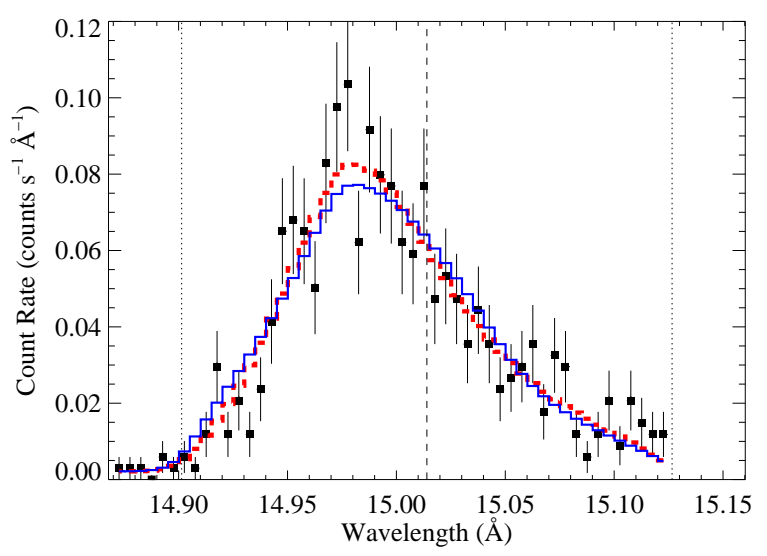

Fig. 34 Two different models that fit the FeXVII line in the CHANDRA spectrum of $\zeta$ Pup. Dashed histogram: non-porous model with $\tau_{*}=2$. Solid histogram: porous model with $\tau_{*}=8$. $h_{\infty}=3.3$. The dashed vertical line refers to the transition frequency at rest and the dotted lines to frequencies corresponding to $\pm v_{\infty}$. See text. (From D. Cohen, priv. comm.)

problem which they named the "near-star high-ion" problem (see also Waldron and Cassinelli 2001). The derived conditions (temperatures $\sim 1-2 \cdot 10^{7} \mathrm{~K}$ close to the star, with $R_{\text {fir }}$ as low as $1.1 R_{*}$ ) would be in stark contrast to the standard shocked-wind scenario, since, e.g., the implied jump velocities would be higher than the velocity of the underlying wind. Moreover, and assuming that the average wind velocity increases with the radius, lines from lower $\mathrm{Z}$ ions should be broader than from ions with higher Z, at least in terms of the standard model of X-ray emission (see below) 47 Contradictory to this expectation, the observed widths of all X-ray lines are similar (e.g., Waldron and Cassinelli 2001, Kramer et al 2003, Waldron and Cassinelli 2007). More investigations are certainly needed to clarify the situation.

Analysis of emission line profiles. Further clues on the X-ray emission from hot stars are provided from the profile shape of strong, single lines (mostly hydrogenlike, e.g., OvIII, NeX, MgXII, SixIV, but also FeXVII), which is asymmetric and skewed. Note first that such a line shape is consistent with the "standard model" (e.g., Hillier et al 1993) which assumes the presence of hot, line-emitting material mixed with a "warm", continuum-absorbing mean wind flow. If the wind is optically thick in the continuum, the emission from the receding hemisphere will be significantly attenuated, i.e., a deficit of red-shifted photons will be observed, whereas from the front, less attenuated side there will be comparatively more emission, contributing to the blue-shifted part. Taken together, an asymmetric profile should be created, already predicted by Macfarlane et al (1991) and indeed observed, as shown in Fig. 34.

Unfortunately, however, this principal agreement was severely challenged after quantitative investigations had been performed. Already from the first analyses on

47 Cassinelli et al (2008) speculate that the "near-star high-ion" problem might be explained by infalling clumps and corresponding bow shocks. 
(Owocki and Cohen 2001, Kramer et al 2003) it turned out that the red parts of the profiles were much less attenuated by the underlying wind than expected. Even worse, the continuum opacity, $\kappa_{v}$, increases with wavelength, and consequently the lines of lower $\mathrm{Z}$ ions are expected to be more skewed compared to the higher $\mathrm{Z}$ ones. Such a dependence, however, has not been found, and the wavelength shifts of line centroids are similar for lines of all ions (Waldron and Cassinelli 2007).

Lower mass-loss rates or porosity/macro-clumping? Different hypotheses to explain these problems have been proposed and are summarized by Oskinova et al (2008). In the following, we will concentrate on two promising alternatives that at least allow for reasonable profile fits, but are still subject to a lively debate (see, e.g., the discussions recorded in Hamann et al 2008b). Both approaches rely on the notion that a reduced continuum opacity can lead to a better agreement between modeled and observed line shapes. This can be achieved either by reducing the adopted mass-loss rate or by accounting for porosity effects. The latter have the additional advantage that the effective opacity, in the optically thick limit, becomes frequency-independent, i.e., grey (Eq. 47), thus potentially explaining the similarity 48 of the line-shapes from different ionization stages.

As a typical example, Fig. 34 displays the observed FexVII profile from $\zeta$ Pup and two fits. The dashed histogram refers to the best fit obtained by Cohen (2008), based on the empirical model by Owocki and Cohen (2001), which optimizes three parameters: the normalization, the inner radius below which there shall be no emission $\left(\mathrm{R}_{\mathrm{o}}\right)$, and the continuum optical depth of the "warm" wind, parameterized by the quantity $\tau_{*}=\kappa_{v} \dot{M} /\left(4 \pi R_{*} v_{\infty}\right)$. The fit is formally good, and the best fit values (within $68 \%$ confidence limits) are $\mathrm{R}_{\mathrm{o}}=1.53_{-0.15}^{+0.12} R_{*}$ and $\tau_{*}=2.0 \pm 0.4$.

Thus, the $R_{0}$ derived from the profile is consistent with the expected onset of shock formation and the optical depth is quite small indeed. Translated to a mass-loss rate, this would imply $\dot{M}=1.5 \cdot 10^{-6} M_{\odot} \mathrm{yr}^{-1}$, which is (much) lower than recent estimates from NLTE analyses based on unclumped models $\left(\dot{M} \approx 8.8 \cdot 10^{-6} M_{\odot} \mathrm{yr}^{-1}\right.$, Repolust et al 2004), and a factor of 2.8 lower than the maximum mass-loss rate $\left(\dot{M}=4.2 \cdot 10^{-6} M_{\odot} \mathrm{yr}^{-1}\right)$ constrained by independent $\rho^{2}$ diagnostics when the outer wind is assumed to be homogeneous (Puls et al 2006, see Sect. 6.3).

Alternative models were simultaneously presented by Oskinova et al (2006) and Owocki and Cohen (2006). Both publications (which differ with respect to important details) suggested that also porosity associated with macro-clumping (Sect. 6.3.3 can account for the low degree of asymmetry, without any need to worry about reduced mass-loss rates. The solid histogram in Fig. 34 bases on the approach by Owocki and Cohen (2006) and displays the best fit for such a porous model, assuming spherical clumps. By fixing the mass-loss rate at $\dot{M}=$ $6 \cdot 10^{-6} M_{\odot} \mathrm{yr}^{-1}$ (corresponding to $\tau_{*}=8$ ), the porosity length, $h$ (Eq. 47) is a free fit-parameter in this model. It is evident that the best fitting porous model with the "high" mass-loss rate is almost indistinguishable from the best fitting non-porous model with the low $\dot{M}$. The optimum terminal porosity length (the value of $h$ in the outer wind region) is rather large, $2.5 R_{*}<h_{\infty}<4 R_{*}$ at a $68 \%$ confidence level. Cohen (2008) argued that this is much larger than any porosity length seen

\footnotetext{
48 The issue of the actual degree of grayness has not been settled though.
} 
in state-of-the-art hydrodynamical simulations, and concluded that "there is no compelling evidence" for invoking porosity rather than a reduced mass-loss rate.

Oskinova et al (2006) pointed out that by combining the fits for $\tau_{*}$ from $\zeta$ Pup, based on non-porous models (from Kramer et al 2003) with appropriate absorption coefficients, different lines would imply significantly different mass-loss rates. Based on previous work by Feldmeier et al (2003) and Oskinova et al (2004), they describe the wind by a flow of clumps obeying the equation of continuity. As in the porosity length formalism, the effective opacity of the clumps becomes grey when the clumps become optically thick. They assume that the emission originates between two radii $r_{1}$ and $r_{2}$ (derived from fir-lines), and investigate the influence of the clump geometry. Comparing spherical clumps with (infinitesimally) thin shell fragments (pancakes) oriented perpendicular to the radial direction, they argue that the latter geometry leads to more symmetric profiles than the former one, as long as the clumps subtend a constant solid angle as they propagate outwards. In their subsequent simulations of synthetic X-ray lines, the shape of the clumps is simulated by an anisotropic opacity (per default assumed to be pancake-like), and the only parameter to be specified (in addition to the wind parameters, $\dot{M}, v_{\infty}$, and $\beta$, adopted from the literature) is the quantity $n_{0}=n(r) v(r)$. This quantity defines the number of fragments passing through some reference radius per unit time (constant due to mass conservation), and was set to $n_{0} \equiv v_{\infty} / R_{*}$, in order to obtain average clump separations $L(r)$ which are compatible with predictions from hydrodynamic simulations, $L(r)=R_{*} v(r) / v_{\infty} \leq R_{*}$. This model has no free parameter, but nevertheless provides a satisfactory agreement between a variety of synthetic and observed lines from different ions (thus supporting the grey character of the opacity). Oskinova et al (2008) conclude that these results provide evidence that the wind clumps are not optically thin, that they are compressed in the radial direction, and that they are separated by a few tenths of the stellar radius in the wind acceleration zone.

Let us finish this section with our conclusions. Whilst the X-ray spectra from magnetic winds seem to confirm the magnetically channeled wind shock scenario, the situation for non-magnetic winds remains unclear. Both non-porous models with reduced mass-loss rates and porous winds with "conventional" $\dot{M}$ are able to explain the observed spectra, where the role of non-isotropic opacity needs further confirmation by independent diagnostics. Future modeling with a simultaneous analysis of processes from the X-ray to the radio domain will certainly uncover the most appropriate description and the "real" mass-loss rates and clarify the "near-star high-ion" problem. Such modeling should include not only the X-ray line-features, but also the SED in the complete X-ray band including the wealth of the ROSAT data.

\section{Summary and further implications}

We reviewed the current status of both theoretical and observational aspects of mass loss from hot massive stars, starting with the standard model and the windmomentum luminosity relation (WLR). Mass-loss predictions from line statistics were discussed with particular emphasis on the temperature and metallicity dependence of the wind momenta, emphasizing that the spectral-type dependence 
of the WLR, especially with respect to the bi-stability jump, is still a most active aspect of present-day hot-star wind research.

We continued with the impact of rotation. Wind compression and the predictions of predominantly prolate winds due to gravity darkening were reviewed, whilst we concluded that the rotationally-induced bi-stability concept for explaining the equatorially outflowing winds from $\mathrm{B}[\mathrm{e}]$ supergiants requires future $2 \mathrm{D}$ modeling. With respect to magnetic fields, we made a clear distinction between internal and surface magnetic fields and note that firm observational constraints on the strength and geometry of the fields have become within reach of current instrumentation (e.g., using ESPADONS or NARVAL). We reviewed the wind structure as a function of the magnetic confinement parameter, and pointed out that weak magnetic fields below present detection limits could have a large impact on weak winds. It remains a possibility that the co-rotating interaction regions that are the likely root of ultraviolet line profile variability of O-stars might be related to an anchored field on the surface.

A key topic concerned stationary models of radiation-driven winds. We described two basic methods in use to predicting the mass-loss rates of massive stars and their pros and cons: hydrodynamical methods based on the improved CAK approach and those based on Monte Carlo radiative transfer methods. Predictions were provided for $\mathrm{O}$ and $\mathrm{B}$ supergiants, followed by those for stars in closer proximity to the Eddington limit, including Luminous Blue Variables (LBVs) and Wolf-Rayet (WR) stars. Furthermore, we provided some basic insights into the possible existence of stellar winds at very low and zero metallicity content (Population III) involving issues such as the potential self-enrichment of metals through the evolution of the first generations of massive stars themselves, and the potential importance of continuum driven winds from super-Eddington stars, which do not require the presence of metals in their atmospheres. Particularly, we considered the predicted metallicity dependence of WR winds since this has relevant implications for the angular momentum evolution of massive stars at low metal content and the progenitor stars of long GRBs.

As far as observational wind diagnostics are concerned, we discussed the traditional $\mathrm{UV}, \mathrm{H}_{\alpha}$, and continuum bound-free/free-free emission, in addition to modern non-LTE atmosphere analyses and emphasized the future potential for NIR spectroscopy. One of the major steps in the last decade has been the inclusion of metal line-blanketing, which has led to a new temperature scale of OB-stars, with generally lower temperatures and luminosities. Due to these lower luminosities the O-star mass-loss predictions per object have been down-revised as a consequence of the changes in underlying stellar parameters - without actually changing the predictions themselves. Empirical mass-loss rates have been determined in parallel with stellar parameters, by profile fitting methods (using the conventional "by eye" method or optimization via genetic algorithms), for a variety of spectral types, luminosity classes and environments, particularly within the VLTFLAMES survey of massive stars. In many cases, the derived mass-loss rates of O-supergiants were a factor of $\sim 2$ above predicted values. This could either mean that the theoretical values are not large enough, or that the empirical values are too high (or that both need revision). For mid and late type B-supergiants, on the other hand, empirical $\dot{M}$-values were found to be lower than the theoretical ones. Comparing the mass-loss rates for the Galaxy, the LMC and the SMC, and accounting 
for an average clumping correction, the empirical dependence of O-star mass-loss rates on metallicity has been derived, which agrees quite well with the theoretical predictions.

In addition to the minor problems outlined in the last paragraph, our general understanding of radiation driven winds is challenged by two urgent problems, the weak wind and the clumping problem. Regarding the former, we have discussed that the presence of X-rays can lead to a degeneracy of mass-loss rates derived from the UV, and suggested the NIR $\mathrm{Br}_{\alpha}$-line as a promising tool for further progress on quantifying mass-loss rates for thin winds. We have also outlined that just these $\mathrm{X}$-rays might be the origin of the weak wind problem, by modifying the ionization equilibrium and thus the efficiency of radiative driving in the lower wind. Future work has to provide tight constraints on the domain of occurrence of weak winds, and particularly on the question which parameters discriminate objects with "normal" from those with weak winds. To be even more provocative: Can we be sure that there are any late O-dwarfs with "normal" winds at all, or did we over-interpret present mass-loss diagnostics, and the weak wind case is the normal one?

In the last part of this review we discussed important issues related to timedependence and structure, featuring the clumping problem. We reported that different diagnostics seem to suggest also different clumping properties (with clumping factors differing by factors up to 20-100), and summarized recent attempts to unify these results by invoking optical depth effects (micro- vs. macro-clumping) and clumping in velocity space. One particular aspect concerns the radial stratification of the clumping. Most analyses seem to have at least one result in common, namely the indication of considerable clumping close to the wind-base. This is in strong contrast to the conventional interpretation of wind-clumping as caused by the line-driven instability (either self-excited or triggered), since all corresponding simulations predict the onset of wind-clumping only at rather large velocities (some hundreds of $\mathrm{km} \mathrm{s}^{-1}$ ). Thus, future effort should be dedicated to improve our understanding of the physical cause of the clumping, but also to continue with empirical studies, by combining evidences from different diagnostics that are formed in different positions in the wind. We have provided a promising "toolkit" for this objective. Of course, the final goal is not only to "measure" clumping/volume filling factors and porosity lengths, but also to accumulate information about the shape of the clumps. Most spectral diagnostics (UV, $\mathrm{H}_{\alpha}, \mathrm{Br}_{\alpha}$, IR-/radio continuum) do not provide such geometric constraints, but we have pointed out that linear polarimetry could help, as well as the diagnostics of X-ray line emission.

Given the overwhelming observational evidence for wind clumping, it is evident that those empirical rates which are based on $\rho^{2}$ diagnostics and homogeneous wind models yield maximum values and that the real mass-loss rates must be lower. Still, the most relevant question is by how much these empirical rates must be down-revised. If the clumping factor is only a factor of $\sim 4$, mass-loss rates would need to be reduced by a corresponding factor of $\sim 2$. Then, at least for O- and early B-stars, empirical rates would be in full agreement with present theoretical rates (still assumed to be uncontaminated by clumping effects), which are used in current models of massive star evolution.

If, on the other hand, the empirical rates needed to be down-revised by very large factors, of 10 or more, they would fall significantly below theoretical rates, 
with some devastating effects on current massive star evolution models. This would tie in with the downwards revision of WR mass-loss rates about a decade ago, with implications involving that black holes have larger masses than previously appreciated, and the possibility that a significant fraction of the total mass loss would need to be lost in super outbursts of LBVs. We note that for a variety of reasons including stellar rotation rates and the origin of WR stars, present day stellar evolution models could not survive with theoretical rates down-revised by more than a factor of two, or, equivalently, empirical rates reduced by more than a factor of four.

Only the future will tell!

Acknowledgements We are indebted to Jean Surdej, Stan Owocki, David Cohen and Ken Gayley for their insightful comments and suggestions. Many thanks to Jon Sundqvist for carefully reading the manuscript and additional suggestions for improvements. F.N. acknowledges a grant by the Spanish MEC through project AYA2007-67546.

\section{References}

Abbott DC (1980) The theory of radiatively driven stellar winds. I - A physical interpretation. ApJ 242:1183-1207

Abbott DC (1982) The theory of radiatively driven stellar winds. II - The line acceleration. ApJ 259:282-301

Abbott DC (1984) The Theory of Radiative Driven Winds from Early-Type Stars. In: Stalio R (ed) Relations Between Chromospheric-Coronal Heating and Mass Loss in Stars, p 265

Abbott DC, Hummer DG (1985) Photospheres of hot stars. I - Wind-blanketed model atmospheres. ApJ 294:286-302

Abbott DC, Lucy LB (1985) Multiline transfer and the dynamics of stellar winds. ApJ 288:679693

Abbott DC, Bieging JH, Churchwell E, Cassinelli JP (1980) VLA radio continuum measurements of mass loss from early-type stars. ApJ 238:196-202

Abbott DC, Bieging JH, Churchwell E (1981a) Mass loss from very luminous OB stars and the Cygnus superbubble. ApJ 250:645-659

Abbott DC, Bieging JH, Churchwell E (1981b) Mass loss from very luminous OB stars and the Cygnus superbubble. ApJ 250:645-659

Abbott DC, Bieging JH, Churchwell E (1984) The detection of variable, nonthermal radio emission from two O type stars. ApJ 280:671-678

Abel T, Bryan GL, Norman ML (2000) The Formation and Fragmentation of Primordial Molecular Clouds. ApJ 540:39-44

Anderson LS (1985) Line blanketing without local thermodynamic equilibrium. I - A hydrostatic stellar atmosphere with hydrogen, helium, and carbon lines. ApJ 298:848-858

Anderson LS (1989) Line blanketing without local thermodynamic equilibrium. II - A solar-type model in radiative equilibrium. ApJ 339:558-578

Auer LH, Mihalas D (1969) Brackett-Alpha Emission in Non-Lte Model Stellar Atmospheres. ApJL 156:L151

Babel J (1995) Multi-component radiatively driven winds from A and B stars. I. The metallic wind of a main sequence A star. A\&A 301:823

Babel J (1996) The fading of radiatively driven winds in B stars. A\&A 309:867-878

Babel J, Montmerle T (1997a) On the Periodic X-Ray Emission from the O7 V Star theta 1 Orionis C. ApJL 485:L29

Babel J, Montmerle T (1997b) X-ray emission from Ap-Bp stars: a magnetically confined windshock model for IQ Aur. A\&A 323:121-138

Baraffe I, Heger A, Woosley SE (2001) On the Stability of Very Massive Primordial Stars. ApJ 550:890-896

Barton EJ, Davé R, Smith JDT, Papovich C, Hernquist L, Springel V (2004) Searching for Star Formation beyond Reionization. ApJL 604:L1-L4 
Bates B, Gilheany S (1990) IUE observations of mass-loss spectral features in B5-B9 supergiants. MNRAS 243:320-329

Bell AR (1978a) The acceleration of cosmic rays in shock fronts. I. MNRAS 182:147-156

Bell AR (1978b) The acceleration of cosmic rays in shock fronts. II. MNRAS 182:443-455

Benaglia P, Vink JS, Martí J, Maíz Apellániz J, Koribalski B, Crowther PA (2007) Testing the predicted mass-loss bi-stability jump at radio wavelengths. A\&A 467:1265-1274

Berghoefer TW, Schmitt JHMM (1994) ROSAT X-ray light curves of early-type stars. Ap\&SS 221:309-320

Berghoefer TW, Baade D, Schmitt JHMM, Kudritzki RP, Puls J, Hillier DJ, Pauldrach AWA (1996) Correlated variability in the X-ray and $\mathrm{H} \alpha$ emission from the O4If supergiant $\zeta$ Puppis. A\&A 306:899

Bianchi L, Garcia M (2002) The Effective Temperatures of Mid-O Stars. ApJ 581:610-625

Bieging JH, Abbott DC, Churchwell EB (1989) A survey of radio emission from Galactic OB stars. ApJ 340:518-536

Bjorkman JE, Cassinelli JP (1993) Equatorial disk formation around rotating stars due to Ram pressure confinement by the stellar wind. ApJ 409:429-449

Blandford RD, Ostriker JP (1978) Particle acceleration by astrophysical shocks. ApJL 221:L29_ L32

Bouret JC, Lanz T, Hillier DJ, Heap SR, Hubeny I, Lennon DJ, Smith LJ, Evans CJ (2003) Quantitative Spectroscopy of O Stars at Low Metallicity: O Dwarfs in NGC 346. ApJ 595:11821205

Bouret JC, Lanz T, Hillier DJ (2005) Lower mass loss rates in O-type stars: Spectral signatures of dense clumps in the wind of two Galactic O4 stars. A\&A 438:301-316

Bouret JC, Hillier DJ, Lanz T (2008) Clumping and Rotation in $\zeta$ Puppis. In: Bresolin F, Crowther PA, Puls J (eds) Massive Stars as Cosmic Engines, IAU Symposium, vol 250

Braithwaite J, Nordlund Å (2006) Stable magnetic fields in stellar interiors. A\&A 450:10771095

Braithwaite J, Spruit HC (2004) A fossil origin for the magnetic field in A stars and white dwarfs. Nature 431:819-821

Bresolin F, Kudritzki RP (2004) Stellar Winds of Hot Massive Stars Nearby and Beyond the Local Group. In: McWilliam A, Rauch M (eds) Origin and Evolution of the Elements, p 283

Bresolin F, Crowther PA, Puls J (eds) (2008) IAU 250 Massive Stars as Cosmic Engines, IAU Symposium, vol 250

Bromm V, Kudritzki RP, Loeb A (2001) Generic Spectrum and Ionization Efficiency of a Heavy Initial Mass Function for the First Stars. ApJ 552:464-472

Brott I, Hunter I, Anders P, Langer N (2008) How Efficient is Rotational Mixing in Massive Stars ? In: First Stars III, American Institute of Physics Conference Series, vol 990, pp 273-275

Carlberg RG (1980) The instability of radiation-driven stellar winds. ApJ 241:1131-1140

Cassinelli JP, MacGregor KB (2000) The Rise of a Magnetic Flux Tube through the Radiative Envelope of a 9 M_solar Star. In: Smith MA, Henrichs HF, Fabregat J (eds) IAU Colloq. 175: The Be Phenomenon in Early-Type Stars, Astronomical Society of the Pacific Conference Series, vol 214, p 337

Cassinelli JP, Cohen DH, Macfarlane JJ, Sanders WT, Welsh BY (1994) X-ray emission from near-main-sequence B stars. ApJ 421:705-717

Cassinelli JP, Brown JC, Maheswaran M, Miller NA, Telfer DC (2002) A Magnetically Torqued Disk Model for Be Stars. ApJ 578:951-966

Cassinelli JP, Ignace R, Waldron W, Cho J, Murphy N, Lazarian A (2008) X-ray line emission produced in clump bow shocks. In: Hamann WR, Oskinova LM, Feldmeier A (eds) Clumping in Hot-Star Winds, pp 217-219

Castor JI (1979) Radiatively-driven winds - Model improvements, ionization balance and the infrared spectrum. In: Conti PS, de Loore CWH (eds) Mass Loss and Evolution of O-Type Stars, IAU Symposium, vol 83, pp 175-189

Castor JI, Abbott DC, Klein RI (1975) Radiation-driven winds in Of stars. ApJ 195:157-174

Chen W, White RL (1994) Nonthermal radio emission from hot star winds: Its origin and physical implications. Ap\&SS 221:259-272

Cherepashchuk AM, Khaliullin KF, Eaton JA (1984) Ultraviolet photometry from the Orbiting Astronomical Observatory. XXXIX - The structure of the eclipsing Wolf-Rayet binary V444 Cygni as derived from light curves between 2460 A and 3. 5 microns. ApJ 281:774-788 
Chesneau O, Roche M, Boccaletti A, Abe L, Moutou C, Charbonnier F, Aime C, Lanteri H, Vakili F (2000) Adaptive optics imaging of P Cygni in Halpha. A\&AS 144:523-532

Chlebowski T, Garmany CD (1991) On winds and X-rays of O-type stars. ApJ 368:241-251

Code A, Whitney B (1995) Polarization from scattering in blobs. ApJ 441:400-407

Cohen DH (2008) X-ray Emission from O Stars. In: Bresolin F, Crowther PA, Puls J (eds) Massive Stars as Cosmic Engines, IAU Symposium, vol 250

Collins GW II (1963) Continuum Emission from a Rapidly Rotating Stellar Atmosphere. ApJ 138:1134

Collins GW II, Harrington JP (1966) Theoretical H-Beta Line Profiles and Related Parameters for Rotating B Stars. ApJ 146:152

Cox P, Mezger PG, Sievers A, Najarro F, Bronfman L, Kreysa E, Haslam G (1995) Millimeter emission of eta Carinae and its surroundings. A\&A 297:168-174

Cranmer SR, Owocki SP (1995) The effect of oblateness and gravity darkening on the radiation driving in winds from rapidly rotating B stars. ApJ 440:308-321

Cranmer SR, Owocki SP (1996) Hydrodynamical Simulations of Corotating Interaction Regions and Discrete Absorption Components in Rotating O-Star Winds. ApJ 462:469

Crowther P (2007) Physical properties of Wolf-Rayet stars. ARA\&A 45:177-219

Crowther PA, Hillier DJ, Evans CJ, Fullerton AW, De Marco O, Willis AJ (2002) Revised Stellar Temperatures for Magellanic Cloud O Supergiants from Far Ultraviolet Spectroscopic Explorer and Very Large Telescope UV-Visual Echelle Spectrograph Spectroscopy. ApJ 579:774-799

Crowther PA, Lennon DJ, Walborn NR (2006) Physical parameters and wind properties of galactic early B supergiants. A\&A 446:279-293

Curé M (2004) The Influence of Rotation in Radiation-driven Wind from Hot Stars: New Solutions and Disk Formation in Be Stars. ApJ 614:929-941

Curé M, Rial DF (2004) The influence of rotation in radiation driven winds from hot stars. II. CAK topological analysis. A\&A 428:545-554

Curé M, Rial DF, Cidale L (2005) Outflowing disk formation in B[e] supergiants due to rotation and bi-stability in radiation driven winds. A\&A 437:929-933

Davidson K (1987) The relation between apparent temperature and mass-loss rate in hypergiant eruptions. ApJ 317:760-764

Davies B, Oudmaijer R, Vink J (2005) Asphericity and clumpiness in the winds of Luminous Blue Variables. A\&A 439:1107-1125

Davies B, Vink J, Oudmaijer R (2007) Modelling the clumping-induced polarimetric variability of hot star winds. A\&A 469:1045-1056

de Araujo FX (1995) The equatorial plane of Be stars: an outflow driven by optically thin lines? A\&A 298:179

De Becker M (2007) Non-thermal emission processes in massive binaries. A\&ARv14:171-216

de Koter A, Lamers HJGLM, Schmutz W (1996) Variability of luminous blue variables. II. Parameter study of the typical LBV variations. A\&A 306:501

de Koter A, Vink J, Muijres L (2008) Constraints on wind clumping form the empirical massloss vs. metallicity relation for early-type stars. In: Hamann WR, Oskinova LM, Feldmeier A (eds) Clumping in Hot-Star Winds, pp 47-49

Dessart L, Owocki SP (2005) 2D simulations of the line-driven instability in hot-star winds. II. Approximations for the 2D radiation force. A\&A 437:657-666

Dessart L, Owocki SP (2002a) Emission profile variability in hot star winds. A pseudo-3D method based on radiation hydrodynamics simulations. A\&A 383:1113-1124

Dessart L, Owocki SP (2002b) Wavelet analysis of instability-generated line profile variations in hot-star winds. A\&A 393:991-996

Dessart L, Owocki SP (2003) Two-dimensional simulations of the line-driven instability in hotstar winds. A\&A 406:L1-L4

Domiciano de Souza A, Kervella P, Jankov S, Abe L, Vakili F, di Folco E, Paresce F (2003) The spinning-top Be star Achernar from VLTI-VINCI. A\&A 407:L47-L50

Donati JF, Semel M, Carter BD, Rees DE, Collier Cameron A (1997) Spectropolarimetric observations of active stars. MNRAS 291:658

Donati JF, Babel J, Harries TJ, Howarth ID, Petit P, Semel M (2002) The magnetic field and wind confinement of $\theta^{1}$ Orionis C. MNRAS 333:55-70

Donati JF, Howarth ID, Jardine MM, Petit P, Catala C, Landstreet JD, Bouret JC, Alecian E, Barnes JR, Forveille T, Paletou F, Manset N (2006) The surprising magnetic topology of $\tau$ 
Sco: fossil remnant or dynamo output? MNRAS 370:629-644

Dougherty SM, Williams PM (2000) Non-thermal emission in Wolf-Rayet stars: are massive companions required? MNRAS 319:1005-1010

Drew JE (1990) A new theoretical calibration of the relation between mass-loss rate and $\mathrm{H}$-alpha emission for O star winds. ApJ 357:573-581

Drew JE, Hoare MG, Denby M (1994) Soft X-ray observations of the early B giants Beta and Epsilon CMa. MNRAS 266:917

Eichler D, Usov V (1993) Particle acceleration and nonthermal radio emission in binaries of early-type stars. ApJ 402:271-279

Eldridge J, Vink J (2006) Implications of the metallicity dependence of Wolf-Rayet winds. A\&A 452:295-301

Evans CJ, Crowther PA, Fullerton AW, Hillier DJ (2004a) Quantitative Studies of the FarUltraviolet, Ultraviolet, and Optical Spectra of Late O- and Early B-Type Supergiants in the Magellanic Clouds. ApJ 610:1021-1037

Evans CJ, Lennon DJ, Trundle C, Heap SR, Lindler DJ (2004b) Terminal Velocities of Luminous, Early-Type Stars in the Small Magellanic Cloud. ApJ 607:451-459

Evans CJ, Smartt SJ, Lee JK, and 23 coauthors (2005) The VLT-FLAMES survey of massive stars: Observations in the Galactic clusters NGC 3293, NGC 4755 and NGC 6611. A\&A 437:467-482

Evans CJ, Lennon DJ, Smartt SJ, Trundle C (2006) The VLT-FLAMES survey of massive stars: observations centered on the Magellanic Cloud clusters NGC 330, NGC 346, NGC 2004, and the N11 region. A\&A 456:623-638

Evans CJ, Hunter I, Smartt SJ, Lennon DJ, de Koter A, Mokiem MR, Trundle C, Dufton PL, Ryans RSI, Puls J, Vink JS, Herrero A, Simon-Diaz S, Langer N, Brott I (2008) The VLTFLAMES Survey of Massive Stars. ArXiv e-prints 803,0803.2820

Eversberg T, Lepine S, Moffat AFJ (1998) Outmoving Clumps in the Wind of the Hot O Supergiant zeta Puppis. ApJ 494:799

Feldmeier A (1995) Time-dependent structure and energy transfer in hot star winds. A\&A 299:523

Feldmeier A (1999) The Line-Driven Instability. In: Wolf B, Stahl O, Fullerton AW (eds) IAU Colloq. 169: Variable and Non-spherical Stellar Winds in Luminous Hot Stars, Lecture Notes in Physics, Berlin Springer Verlag, vol 523, p 285

Feldmeier A, Shlosman I (2000) Runaway of Line-driven Winds toward Critical and Overloaded Solutions. ApJL 532:L125-L128

Feldmeier A, Shlosman I (2002) Abbott Wave-triggered Runaway in Line-driven Winds from Stars and Accretion Disks. ApJ 564:385-394

Feldmeier A, Puls J, Pauldrach AWA (1997) A possible origin for X-rays from O stars. A\&A 322:878-895

Feldmeier A, Oskinova L, Hamann WR (2003) X-ray line emission from a fragmented stellar wind. A\&A 403:217-224

Fermi E (1949) On the Origin of the Cosmic Radiation. Physical Review 75:1169-1174

Figer DF, Najarro F, Morris M, McLean IS, Geballe TR, Ghez AM, Langer N (1998) The Pistol Star. ApJ 506:384-404

Figer DF, Najarro F, Gilmore D, Morris M, Kim SS, Serabyn E, McLean IS, Gilbert AM, Graham JR, Larkin JE, Levenson NA, Teplitz HI (2002) Massive Stars in the Arches Cluster. ApJ 581:258-275

Foellmi C, Moffat AFJ, Guerrero MA (2003a) Wolf-Rayet binaries in the Magellanic Clouds and implications for massive-star evolution - I. Small Magellanic Cloud. MNRAS 338:360-388

Foellmi C, Moffat AFJ, Guerrero MA (2003b) Wolf-Rayet binaries in the Magellanic Clouds and implications for massive-star evolution - II. Large Magellanic Cloud. MNRAS 338:10251056

Friend DB, Abbott DC (1986) The theory of radiatively driven stellar winds. III - Wind models with finite disk correction and rotation. ApJ 311:701-707

Friend DB, Castor JI (1983) Stellar winds driven by multiline scattering. ApJ 272:259-272

Fullerton AW (1999) Rotationally Modulated Winds of O Stars. In: Wolf B, Stahl O, Fullerton AW (eds) IAU Colloq. 169: Variable and Non-spherical Stellar Winds in Luminous Hot Stars, Lecture Notes in Physics, Berlin Springer Verlag, vol 523, p 3

Fullerton AW, Owocki SP (1992) Can Nonstationary Velocity Plateaus Account for Slowly Moving Discrete Absorption Components? (Contributed Poster). In: Drissen L, Leitherer C, Nota 
A (eds) Nonisotropic and Variable Outflows from Stars, Astronomical Society of the Pacific Conference Series, vol 22, p 177

Fullerton AW, Gies DR, Bolton CT (1996) Absorption Line Profile Variations among the O Stars. I. The Incidence of Variability. ApJS 103:475

Fullerton AW, Massa DL, Prinja RK, Owocki SP, Cranmer SR (1997) Wind variability of B supergiants. III. Corotating spiral structures in the stellar wind of HD 64760. A\&A 327:699720

Fullerton AW, Massa DL, Prinja RK (2006) The Discordance of Mass-Loss Estimates for Galactic O-Type Stars. ApJ 637:1025-1039

Fullerton AW, Massa DL, Prinja RK (2008) Revised mass-loss rates for O stars from the Pv resonance line. In: Hamann WR, Oskinova LM, Feldmeier A (eds) Clumping in Hot-Star Winds, pp 23-26

Gabler A, Gabler R, Pauldrach A, Puls J, Kudritzki RP (1990) On the use of H-alpha and He II 4686 as mass-loss and luminosity indicators for hot stars. In: Garmany CD (ed) Properties of Hot Luminous Stars, Astronomical Society of the Pacific Conference Series, vol 7, pp 218-229

Gabler R, Gabler A, Kudritzki RP, Puls J, Pauldrach A (1989) Unified NLTE model atmospheres including spherical extension and stellar winds - Method and first results. A\&A 226:162182

Gabriel AH, Jordan C (1969) Interpretation of solar helium-like ion line intensities. MNRAS 145:241

Gagné M, Oksala ME, Cohen DH, Tonnesen SK, ud-Doula A, Owocki SP, Townsend RHD, MacFarlane JJ (2005) Chandra HETGS Multiphase Spectroscopy of the Young Magnetic O Star $\theta^{1}$ Orionis C. ApJ 628:986-1005

Garcia M (2005) A Study of Massive Stars from High Resolution Spectroscopy in the Far-UV and UV. PhD thesis, University of La Laguna (Teneriffe)

Garcia M, Bianchi L (2004) The Effective Temperatures of Hot Stars. II. The Early-O Types. ApJ 606:497-513

Gautschy A, Glatzel W (1990) On Highly Non-Adiabatic Stellar Pulsations and the Origin of Strange Modes. MNRAS 245:597

Gayley KG (1995) An Improved Line-Strength Parameterization in Hot-Star Winds. ApJ 454:410

Gayley KG (2000) The Surprisingly Weak Effect of Gravity in Retarding Hot-Star Wind Acceleration. ApJ 529:1019-1025

Gayley KG, Owocki SP (1994) Acceleration efficiency in line-driven flows. ApJ 434:684-694

Gayley KG, Owocki SP, Cranmer SR (1995) Momentum deposition on Wolf-Rayet winds: Nonisotropic diffusion with effective gray opacity. ApJ 442:296-310

Geballe TR, Najarro F, Figer DF (2000) A Second Luminous Blue Variable in the Quintuplet Cluster. ApJL 530:L97-L101

Geballe TR, Najarro F, Rigaut F, Roy JR (2006) The K-Band Spectrum of the Hot Star in IRS 8: An Outsider in the Galactic Center? ApJ 652:370-375

Georgiev LN, Hillier DJ, Zsargó J (2006) 2D non-LTE modeling for axisymmetric winds. Method and test cases. A\&A 458:597-608

Gieren W, Pietrzynski G, Bresolin F, Kudritzki RP, Minniti D, Urbaneja M, Soszynski I, Storm J, Fouque P, Bono G, Walker A, Garcia J (2005) Measuring Improved Distances to Nearby Galaxies: The Araucaria Project. The Messenger 121:23-28

Glatzel W (1998) Remarks on the "Omega -limit". A\&A 339:L5-L8

Glatzel W, Chernigovski S (2001) Strange-mode instabilities in the nonlinear regime. In: de Groot M, Sterken C (eds) P Cygni 2000: 400 Years of Progress, Astronomical Society of the Pacific Conference Series, vol 233, p 227

Glatzel W, Kiriakidis M, Fricke KJ (1993) On the stability and pulsations of Wolf-Rayet stars. MNRAS 262:L7-L11

Grady CA, Snow TP Jr, Timothy JG (1983) Observations of Of-star wind variability. ApJ 271:691-701

Gräfener G, Hamann WR (2005) Hydrodynamic model atmospheres for WR stars. Selfconsistent modeling of a WC star wind. A\&A 432:633-645

Gräfener G, Hamann WR (2008) Mass loss from late-type WN stars and its Z-dependence. Very massive stars approaching the Eddington limit. A\&A 482:945-960

Gräfener G, Koesterke L, Hamann WR (2002) Line-blanketed model atmospheres for WR stars. 
A\&A 387:244-257

Groenewegen MAT, Lamers HJGLM (1989) The winds of O-stars. I - an analysis of the UV line profiles with the SEI method. A\&AS 79:359-383

Groenewegen MAT, Lamers HJGLM, Pauldrach AWA (1989) The winds of O-stars. II - The terminal velocities of stellar winds of O-type stars. A\&A 221:78-88

Groote D, Schmitt JHMM (2004) Discovery of X-ray flaring on the magnetic Bp-star $\sigma$ Ori E. A\&A 418:235-242

Hamann WR (1980) The expanding envelope of Zeta Puppis - A detailed UV-line fit. A\&A 84:342-349

Hamann WR (1981a) Line formation in expanding atmospheres - On the validity of the Sobolev approximation. A\&A 93:353-361

Hamann WR (1981b) The expanding envelope of Tau Scorpii - A detailed UV-line fit. A\&A 100:169-174

Hamann WR, Koesterke L (1998) Spectrum formation in clumped stellar winds: consequences for the analyses of Wolf-Rayet spectra. A\&A 335:1003-1008

Hamann WR, Brown JC, Feldmeier A, Oskinova LM (2001) On the wavelength drift of spectral features from structured hot star winds. A\&A 378:946-953

Hamann WR, Feldmeier A, Oskinova LM (2008a) Spectrum formation in clumpy stellar winds. In: Hamann WR, Oskinova LM, Feldmeier A (eds) Clumping in Hot-Star Winds, pp 75-80

Hamann WR, Oskinova LM, Feldmeier A (eds) (2008b) Clumping in Hot-Star Winds, Potsdam: Universitätsverlag Potsdam

Harries T (2000) Synthetic line profiles of rotationally distorted hot-star winds. MNRAS 315:722-734

Harries T, Hillier DJ, Howarth ID (1998) A spectropolarimetric survey of northern hemisphere Wolf-Rayet stars. MNRAS 296:1072-1088

Haser SM (1995) UV spectroscopy of hot stars in the Local Group (in German). PhD thesis, Ludwig-Maximilians-Universität München

Haser SM, Pauldrach AWA, Lennon DJ, Kudritzki RP, Lennon M, Puls J, Voels SA (1998) Quantitative UV spectroscopy of early O stars in the Magellanic Clouds. The determination of the stellar metallicities. A\&A 330:285-305

Hauschildt PH (1992) A fast operator perturbation method for the solution of the special relativistic equation of radiative transfer in spherical symmetry. Journal of Quantitative Spectroscopy and Radiative Transfer 47:433-453

Heap SR, Lanz T, Hubeny I (2006) Fundamental Properties of O-Type Stars. ApJ 638:409-432

Heger A, Woosley SE, Spruit HC (2005) Presupernova Evolution of Differentially Rotating Massive Stars Including Magnetic Fields. ApJ 626:350-363

Henrichs H (1991) Why Are Winds of O Stars Variable? In: Baade D (ed) Rapid Variability of OB-stars: Nature and Diagnostic Value, p 199

Henrichs HF, Kaper L, Zwarthoed GAA (1988) Rapid variability in O star winds. In: Rolfe EJ (ed) A Decade of UV Astronomy with the IUE Satellite, Volume 2, vol 2, pp 145-149

Herrero A, Puls J, Villamariz MR (2000) Fundamental parameters of Galactic luminous OB stars. IV. The upper HR diagram. A\&A 354:193-215

Herrero A, Puls J, Najarro F (2002) Fundamental parameters of Galactic luminous OB stars VI. Temperatures, masses and WLR of Cyg OB2 supergiants. A\&A 396:949-966

Hillier DJ (1991) The effects of electron scattering and wind clumping for early emission line stars. A\&A 247:455-468

Hillier DJ, Allen DA (1992) A spectroscopic investigation of Eta Carinae and the Homunculus Nebula. I - Overview of the spectra. A\&A 262:153-170

Hillier DJ, Miller DL (1998) The Treatment of Non-LTE Line Blanketing in Spherically Expanding Outflows. ApJ 496:407

Hillier DJ, Miller DL (1999) Constraints on the Evolution of Massive Stars through Spectral Analysis. I. The WC5 Star HD 165763. ApJ 519:354-371

Hillier DJ, Kudritzki RP, Pauldrach AW, Baade D, Cassinelli JP, Puls J, Schmitt JHMM (1993) The 0.1-2.5-KEV X-Ray Spectrum of the O4F-STAR Zeta-Puppis. A\&A 276:117

Hillier DJ, Crowther PA, Najarro F, Fullerton AW (1998) An optical and near-IR spectroscopic study of the extreme P Cygni-type supergiant HDE 316285. A\&A 340:483-496

Hillier DJ, Davidson K, Ishibashi K, Gull T (2001) On the Nature of the Central Source in $\eta$ Carinae. ApJ 553:837-860

Hillier DJ, Lanz T, Heap SR, Hubeny I, Smith LJ, Evans CJ, Lennon DJ, Bouret JC (2003) A 
Tale of Two Stars: The Extreme O7 Iaf+ Supergiant AV 83 and the OC7.5 III((f)) star AV 69. ApJ 588:1039-1063

Hirschi R (2008) The impact of reduced mass loss rates on the evolution of massive stars. In: Hamann WR, Oskinova LM, Feldmeier A (eds) Clumping in Hot-Star Winds, pp 9-14

Howarth ID, Prinja RK (1989) The stellar winds of 203 Galactic O stars - A quantitative ultraviolet survey. ApJS 69:527-592

Holzer TE (1977) Effects of rapidly diverging flow, heat addition, and momentum addition in the solar wind and stellar winds. J Geophys Res 82:23-35

Howarth ID (1992) Intrinsic Stellar-Wind Variability (Invited Paper). In: Drissen L, Leitherer C, Nota A (eds) Nonisotropic and Variable Outflows from Stars, Astronomical Society of the Pacific Conference Series, vol 22, p 155

Howarth ID, Prinja RK, Massa D (1995) The IUE MEGA Campaign: The Rotationally Modulated Wind of zeta Puppis. ApJL 452:L65

Hubeny I (1998) Non-LTE line-blanketed model atmospheres of hot stars. In: Chan KL, Cheng KS, Singh HP (eds) 1997 Pacific Rim Conference on Stellar Astrophysics, Astronomical Society of the Pacific Conference Series, vol 138, p 139

Hultzsch PJN, Puls J, Méndez RH, Pauldrach AWA, Kudritzki RP, Hoffmann TL, McCarthy JK (2007) Central stars of planetary nebulae in the Galactic bulge. A\&A 467:1253-1264

Ignace R, Oskinova L (1999) An explanation of observed trends in the X-ray emission from single Wolf-Rayet stars. A\&A 348:L45-L48

Johnson JL, Greif TH, Bromm V (2008) The First Stars. In: F Bresolin PC, Puls J (eds) IAU 250 Massive Stars as Cosmic Engines

Kaper L, Henrichs HF, Fullerton AW, Ando H, Bjorkman KS, Gies DR, Hirata R, Kambe E, McDavid D, Nichols JS (1997) Coordinated ultraviolet and $\mathrm{H} \alpha$ spectroscopy of bright Otype stars. A\&A 327:281-298

Kaper L, Henrichs HF, Nichols JS, Telting JH (1999) Long- and short-term variability in O-star winds. II. Quantitative analysis of DAC behaviour. A\&A 344:231-262

Kato M (1985) A new type of solution for very massive stars and the occurrence of steady mass loss. PASJ 37:311-323

Kaufer A, Stahl O, Wolf B, Gaeng T, Gummersbach CA, Kovacs J, Mandel H, Szeifert T (1996) Long-term spectroscopic monitoring of BA-type supergiants. I. $\mathrm{H}_{\alpha}$ line-profile variability. A\&A 305:887

Kaufer A, Stahl O, Prinja RK, Witherick D (2006) Multi-periodic photospheric pulsations and connected wind structures in ${ }_{i}$ ASTROBJ $_{i}$ HD $64760_{i} /$ ASTROBJ $_{i}$. A\&A 447:325-341

Klein RI, Castor JI (1978) H and He II spectra of Of stars. ApJ 220:902-923

Kobulnicky HA, Fryer CL (2007) A New Look at the Binary Characteristics of Massive Stars. ApJ 670:747-765

Kotak R, Vink JS (2006) Luminous blue variables as the progenitors of supernovae with quasiperiodic radio modulations. A\&A 460:L5-L8

Kramer RH, Cohen DH, Owocki SP (2003) X-Ray Emission-Line Profile Modeling of O Stars: Fitting a Spherically Symmetric Analytic Wind-Shock Model to the Chandra Spectrum of $\zeta$ Puppis. ApJ 592:532-538

Krtička J (2006) NLTE models of line-driven stellar winds - II. O stars in the Small Magellanic Cloud. MNRAS p 266

Krtička J, Kubát J (2000) Isothermal two-component stellar wind of hot stars. A\&A 359:983990

Krtička J, Kubat J (2006) The winds of hot massive first stars. A\&A 446:1039-1049

Krtička J, Owocki SP, Kubát J, Galloway RK, Brown JC (2003) On multicomponent effects in stellar winds of stars at extremely low metallicity. A\&A 402:713-718

Krtička J, Puls J, Kubat J (2008) The influence of clumping on predicted O star wind parameters. In: Hamann WR, Oskinova LM, Feldmeier A (eds) Clumping in Hot-Star Winds, pp 111114

Kudritzki RP (2002) Line-driven Winds, Ionizing Fluxes, and Ultraviolet Spectra of Hot Stars at Extremely Low Metallicity. I. Very Massive O Stars. ApJ 577:389-408

Kudritzki RP, Puls J (2000) Winds from Hot Stars. ARA\&A 38:613-666

Kudritzki RP, The GSMT Science Working Group (2003) GSMT Science Working Group Report http://www.aura-nio.noao.edu

Kudritzki RP, Pauldrach A, Puls J (1987) Radiation driven winds of hot luminous stars. II - Wind models for O-stars in the Magellanic Clouds. A\&A 173:293-298 
Kudritzki RP, Pauldrach A, Puls J, Abbott DC (1989) Radiation-driven winds of hot stars. VI Analytical solutions for wind models including the finite cone angle effect. A\&A 219:205218

Kudritzki RP, Puls J, Gabler R, Schmitt JHMM (1991) Hot Stars - what can BE Learned from Extreme Ultraviolet Spectroscopy. In: Malina RF, Bowyer S (eds) Extreme Ultraviolet Astronomy, p 130

Kudritzki RP, Hummer DG, Pauldrach AWA, Puls J, Najarro F, Imhoff J (1992) Radiation-driven winds of hot luminous stars. $\mathrm{X}$ - The determination of stellar masses, radii and distances from terminal velocities and mass-loss rates. A\&A 257:655-662

Kudritzki RP, Lennon DJ, Puls J (1995) Quantitative Spectroscopy of Luminous Blue Stars in Distant Galaxies. In: Walsh JR, Danziger IJ (eds) Science with the VLT, p 246

Kudritzki RP, Palsa R, Feldmeier A, Puls J, Pauldrach AWA (1996) The X-ray emission from O stars. In: Zimmermann HU, Trümper J, Yorke H (eds) Roentgenstrahlung from the Universe, pp 9-12

Kudritzki RP, Mendez RH, Puls J, McCarthy JK (1997) Winds in the atmospheres of central stars of Planetary Nebulae (Invited Review). In: Habing HJ, Lamers HJGLM (eds) Planetary Nebulae, IAU Symposium, vol 180, p 64

Kudritzki RP, Puls J, Lennon DJ, Venn KA, Reetz J, Najarro F, McCarthy JK, Herrero A (1999) The wind momentum-luminosity relationship of galactic A- and B-supergiants. A\&A 350:970-984

Kudritzki RP, Bresolin F, Przybilla N (2003) A New Extragalactic Distance Determination Method Using the Flux-weighted Gravity of Late B and Early A Supergiants. ApJL 582:L83-L86

Kudritzki RP, Urbaneja MA, Puls J (2006) Atmospheres and Winds of PN Central Stars. In: Barlow MJ, Méndez RH (eds) Planetary Nebulae in our Galaxy and Beyond, IAU Symposium, vol 234, pp 119-126

Kudritzki RP, Urbaneja MA, Bresolin F, Przybilla N (2008a) Extragalactic Stellar Astronomy with the Brightest Stars in the Universe. In: Bresolin F, Crowther PA, Puls J (eds) Massive Stars as Cosmic Engines, IAU Symposium, vol 250

Kudritzki RP, Urbaneja MA, Bresolin F, Przybilla N, Gieren W, Pietrzynski G (2008b) Quantitative Spectroscopy of 24 A supergiants in the Sculptor galaxy NGC 300: Flux weighted gravity luminosity relationship, metallicity and metallicity gradient. ArXiv e-prints 803, 0803.3654

Lamers HJG, Pauldrach AWA (1991) The formation of outflowing disks around early-type stars by bi-stable radiation-driven winds. A\&A 244:L5-L8

Lamers HJGLM, Leitherer C (1993) What are the mass-loss rates of O stars? ApJ 412:771-791

Lamers HJGLM, Morton DC (1976) Mass ejection from the O4f star Zeta Puppis. ApJS 32:715736

Lamers HJGLM, Waters LBFM (1984a) The infrared and radio spectrum of early-type stars with mass loss. I - The curve-of-growth method. A\&A 136:37-52

Lamers HJGLM, Waters LBFM (1984b) The infrared and radio spectrum of early type stars with mass loss. III - The effect of coronae, hot blobs and clumping on the IR and radio spectrum. A\&A 138:25-35

Lamers HJGLM, Gathier R, Snow TP Jr (1982) Narrow components in the profiles of ultraviolet resonance lines - Evidence for a two-component stellar wind for $\mathrm{O}$ and B stars. ApJ 258:186-200

Lamers HJGLM, Waters LBFM, Wesselius PR (1984) The IRAS infrared spectrum of Zeta Puppis (O4If). A\&A 134:L17-L20

Lamers HJGLM, Cerruti-Sola M, Perinotto M (1987) The 'SEI' method for accurate and efficient calculations of line profiles in spherically symmetric stellar winds. ApJ 314:726-738

Lamers HJGLM, Snow TP, Lindholm DM (1995) Terminal Velocities and the Bistability of Stellar Winds. ApJ 455:269

Lamers HJGLM, Zickgraf FJ, de Winter D, Houziaux L, Zorec J (1998) An improved classification of B[e]-type stars. A\&A 340:117-128

Lamers HJGLM, Vink JS, de Koter A, Cassinelli JP (1999) Disks formed by Rotation Induced Bi-Stability. In: Wolf B, Stahl O, Fullerton AW (eds) IAU Colloq. 169: Variable and Nonspherical Stellar Winds in Luminous Hot Stars, Lecture Notes in Physics, Berlin Springer Verlag, vol 523, p 159

Langer N (1997) The Eddington Limit in Rotating Massive Stars. In: Nota A, Lamers H (eds) 
Luminous Blue Variables: Massive Stars in Transition, Astronomical Society of the Pacific Conference Series, vol 120, p 83

Langer N (1998) Coupled mass and angular momentum loss of massive main sequence stars. A\&A 329:551-558

Langer N, Hamann WR, Lennon M, Najarro F, Pauldrach AWA, Puls J (1994) Towards an understanding of very massive stars. A new evolutionary scenario relating O stars, LBVs and Wolf-Rayet stars. A\&A 290:819-833

Langer N, Heger A, Fliegner J (1997) Rotation: a fundamental parameter of massive stars. In: Bedding TR, Booth AJ, Davis J (eds) IAU Symposium, IAU Symposium, vol 189, pp 343348

Lefever K (2007) Fundamental parameters of B-type stars - Application to a HIPPARCOS sample of B supergiants and a CoRoT sample of B dwarfs. PhD thesis, Catholic University Leuven, Belgium

Lefever K, Puls J, Aerts C (2007) Statistical properties of a sample of periodically variable Btype supergiants. Evidence for opacity-driven gravity-mode oscillations. A\&A 463:10931109

Leitherer C (1988) H-alpha as a tracer of mass loss from OB stars. ApJ 326:356-367

Leitherer C, Schmutz W, Abbott DC, Hamann WR, Wessolowski U (1989) Atmospheric models for luminous blue variables. ApJ 346:919-931

Lenorzer A, Mokiem MR, de Koter A, Puls J (2004) Modeling the near-infrared lines of O-type stars. A\&A 422:275-288

Lepine S, Moffat AFJ (1999) Wind Inhomogeneities in Wolf-Rayet Stars. II. Investigation of Emission-Line Profile Variations. ApJ 514:909-931

Lepine S, Moffat AFJ (2008) Direct spectroscopic observations of clumping in O-star winds. ArXiv e-prints 805,0805.1864

Leutenegger MA, Paerels FBS, Kahn SM, Cohen DH (2006) Measurements and Analysis of Helium-like Triplet Ratios in the X-Ray Spectra of O-Type Stars. ApJ 650:1096-1110

Leutenegger MA, Owocki SP, Kahn SM, Paerels FBS (2007) Evidence for the Importance of Resonance Scattering in X-Ray Emission Line Profiles of the O Star $\zeta$ Puppis. ApJ 659:642-649

Litvinenko YE (2003) Particle Acceleration by Magnetic Reconnection. In: Klein L (ed) Energy Conversion and Particle Acceleration in the Solar Corona, Lecture Notes in Physics, Berlin Springer Verlag, vol 612, pp 213-229

Lobel A, Blomme R (2008) Modeling Ultraviolet Wind Line Variability in Massive Hot Stars. ApJ 678:408-430

Lucy LB (1982) The formation of resonance lines in locally nonmonotonic winds. ApJ 255:278285

Lucy LB (1983) The formation of resonance lines in locally nonmonotonic winds. II - an amplitude diagnostic. ApJ 274:372-379

Lucy LB (1984) Wave amplification in line-driven winds. ApJ 284:351-356

Lucy LB (1998) Thirty Years of Radiation-Driven Hot Star Winds. In: Kaper L, Fullerton AW (eds) Cyclical Variability in Stellar Winds, p 16

Lucy LB (2001) An iterative technique for solving equations of statistical equilibrium. MNRAS 326:95-101

Lucy LB (2007a) Mass fluxes for O stars. A\&A 468:649-655

Lucy LB (2007b) The structure of line-driven winds. A\&A 474:701-706

Lucy LB, Abbott DC (1993) Multiline transfer and the dynamics of Wolf-Rayet winds. ApJ 405:738-746

Lucy LB, Solomon PM (1970) Mass Loss by Hot Stars. ApJ 159:879-893

Lucy LB, White RL (1980) X-ray emission from the winds of hot stars. ApJ 241:300-305

Lupie O, Nordsieck K (1987) Visible and infrared continuum spectropolarimetric observations of ten OB supergiant and O emission-line stars. Astron Nachr 93:214-230

MacDonald J, Mullan DJ (2004) Magnetic fields in massive stars: dynamics and origin. MNRAS 348:702-716

Macfarlane JJ, Cassinelli JP, Welsh BY, Vedder PW, Vallerga JV, Waldron WL (1991) Predicted extreme-ultraviolet line emission for nearby main-sequence B stars. ApJ 380:564-574

Macfarlane JJ, Waldron WL, Corcoran MF, Wolff MJ, Wang P, Cassinelli JP (1993) Effects of Coronal and Shock-produced X-Rays on the Ionization Distribution in Hot Star Winds. ApJ 419:813 
MacGregor KB, Hartmann L, Raymond JC (1979) Radiative amplification of sound waves in the winds of $\mathrm{O}$ and B stars. ApJ 231:514-523

Madura TI, Owocki SP, Feldmeier A (2007) A Nozzle Analysis of Slow-Acceleration Solutions in One-dimensional Models of Rotating Hot-Star Winds. ApJ 660:687-698

Maeder A (1999) Stellar evolution with rotation IV: von Zeipel's theorem and anisotropic losses of mass and angular momentum. A\&A 347:185-193

Maeder A, Eenens P (eds) (2004) Stellar Rotation, IAU Symposium, vol 215

Maeder A, Meynet G (2000a) Stellar evolution with rotation. VI. The Eddington and Omega -limits, the rotational mass loss for OB and LBV stars. A\&A 361:159-166

Maeder A, Meynet G (2000b) The Evolution of Rotating Stars. ARA\&A 38:143-190

Maeder A, Meynet G (2003a) Stellar evolution with rotation and magnetic fields. I. The relative importance of rotational and magnetic effects. A\&A 411:543-552

Maeder A, Meynet G (2003b) The role of rotation and mass loss in the evolution of massive stars. In: van der Hucht K, Herrero A, Esteban C (eds) A Massive Star Odyssey: From Main Sequence to Supernova, IAU Symposium, vol 212, p 267

Maeder A, Meynet G (2004) Stellar evolution with rotation and magnetic fields. II. General equations for the transport by Tayler-Spruit dynamo. A\&A 422:225-237

Maeder A, Meynet G (2005) Stellar evolution with rotation and magnetic fields. III. The interplay of circulation and dynamo. A\&A 440:1041-1049

Maoz D (2008) On the fraction of intermediate-mass close binaries that explode as Type Ia supernovae. MNRAS 384:267-277

Markova N (1986) The ejection of shells in the stellar wind of P CYG - The most plausible explanation of the Balmer-line radial velocity variations. A\&A 162:L3-L5

Markova N, Puls J (2008) Bright OB stars in the Galaxy. IV. Stellar and wind parameters of early to late B supergiants. A\&A 478:823-842

Markova N, Puls J, Repolust T, Markov H (2004) Bright OB stars in the Galaxy. I. Mass-loss and wind-momentum rates of O-type stars: A pure $\mathrm{H} \alpha$ analysis accounting for line-blanketing. A\&A 413:693-709

Markova N, Puls J, Scuderi S, Markov H (2005) Bright OB stars in the Galaxy. II. Wind variability in $\mathrm{O}$ supergiants as traced by $\mathrm{H} \alpha$. A\&A 440:1133-1151

Martins F, Schaerer D, Hillier DJ (2002) On the effective temperature scale of O stars. A\&A 382:999-1004

Martins F, Schaerer D, Hillier DJ, Heydari-Malayeri M (2004) Puzzling wind properties of young massive stars in SMC-N81. A\&A 420:1087-1106

Martins F, Schaerer D, Hillier DJ (2005a) A new calibration of stellar parameters of Galactic O stars. A\&A 436:1049-1065

Martins F, Schaerer D, Hillier DJ, Meynadier F, Heydari-Malayeri M, Walborn NR (2005b) O stars with weak winds: the Galactic case. A\&A 441:735-762

Martins F, Genzel R, Hillier DJ, Eisenhauer F, Paumard T, Gillessen S, Ott T, Trippe S (2007) Stellar and wind properties of massive stars in the central parsec of the Galaxy. A\&A 468:233-254

Massa D, Fullerton AW, Nichols JS, Owocki SP, Prinja RK, and 28 co-authors (1995) The IUE MEGA Campaign: Wind Variability and Rotation in Early-Type Stars. ApJL 452:L53

Massey P, Bresolin F, Kudritzki RP, Puls J, Pauldrach AWA (2004) The Physical Properties and Effective Temperature Scale of O-Type Stars as a Function of Metallicity. I. A Sample of 20 Stars in the Magellanic Clouds. ApJ 608:1001-1027

Massey P, Puls J, Pauldrach AWA, Bresolin F, Kudritzki RP, Simon T (2005) The Physical Properties and Effective Temperature Scale of O-Type Stars as a Function of Metallicity. II. Analysis of 20 More Magellanic Cloud Stars and Results from the Complete Sample. ApJ 627:477-519

McErlean ND, Lennon DJ, Dufton PL (1999) Galactic B-supergiants: A non-LTE model atmosphere analysis to estimate atmospheric parameters and chemical compositions. A\&A 349:553-572

Meynet G, Maeder A (2000) Stellar evolution with rotation. V. Changes in all the outputs of massive star models. A\&A 361:101-120

Meynet G, Maeder A, Schaller G, Schaerer D, Charbonnel C (1994) Grids of massive stars with high mass loss rates. V. From 12 to 120 Msun at $\mathrm{Z}=0.001,0.004,0.008,0.020$ and 0.040 . A\&AS 103:97-105

Meynet G, Ekström S, Maeder A (2006) The early star generations: the dominant effect of 
rotation on the CNO yields. A\&A 447:623-639

Milne EA (1926) On the possibility of the emission of high-speed atoms from the sun and stars. MNRAS 86:459-473

Moffat AFJ, Drissen L, Lamontagne R, Robert C (1988) Spectroscopic evidence for rapid blob ejection in Wolf-Rayet stars. ApJ 334:1038-1043

Moffat AFJ, Marchenko SV, Zhilyaev BE, Rowe JF, Muntean V, Chené AN, Matthews JM, Kuschnig R, Guenther DB, Rucinski SM, Sasselov D, Walker GAH, Weiss WW (2008) MOST Finds No Coherent Oscillations in the Hot Carbon-rich Wolf-Rayet Star HD 165763 (WR 111). ApJL 679:L45-L48

Mokiem MR, de Koter A, Puls J, Herrero A, Najarro F, Villamariz MR (2005) Spectral analysis of early-type stars using a genetic algorithm based fitting method. A\&A 441:711-733

Mokiem MR, de Koter A, Evans CJ, Puls J, Smartt SJ, Crowther PA, Herrero A, Langer N, Lennon DJ, Najarro F, Villamariz MR, Yoon SC (2006) The VLT-FLAMES survey of massive stars: mass loss and rotation of early-type stars in the SMC. A\&A 456:1131-1151

Mokiem MR, de Koter A, Evans CJ, Puls J, Smartt SJ, Crowther PA, Herrero A, Langer N, Lennon DJ, Najarro F, Villamariz MR, Vink JS (2007a) The VLT-FLAMES survey of massive stars: wind properties and evolution of hot massive stars in the Large Magellanic Cloud. A\&A 465:1003-1019

Mokiem MR, de Koter A, Vink JS, Puls J, Evans CJ, Smartt SJ, Crowther PA, Herrero A, Langer N, Lennon DJ, Najarro F, Villamariz MR (2007b) The empirical metallicity dependence of the mass-loss rate of O- and early B-type stars. A\&A 473:603-614

Monnier JD, Zhao M, Pedretti E, Thureau N, Ireland M, Muirhead P, Berger JP, Millan-Gabet R, Van Belle G, ten Brummelaar T, McAlister H, Ridgway S, Turner N, Sturmann L, Sturmann J, Berger D (2007) Imaging the Surface of Altair. Science 317:342-345

Morel T, Butler K, Aerts C, Neiner C, Briquet M (2006) Abundance analysis of prime B-type targets for asteroseismology. I. Nitrogen excess in slowly-rotating $\beta$ Cephei stars. A\&A 457:651-663

Morton DC, Underhill AB (1977) The ultraviolet spectrum of Zeta Puppis. ApJS 33:83-99

Moss D (2001) Magnetic Fields in the Ap and Bp Stars: a Theoretical Overview. In: Mathys G, Solanki SK, Wickramasinghe DT (eds) Magnetic Fields Across the Hertzsprung-Russell Diagram, Astronomical Society of the Pacific Conference Series, vol 248, p 305

Mullan DJ (1984) Corotating interaction regions in stellar winds. ApJ 283:303-312

Mullan DJ (1986) Displaced narrow absorption components in the spectra of mass-losing OB stars - Indications of corotating interaction regions? A\&A 165:157-162

Mullan DJ, MacDonald J (2005) Dynamo-generated magnetic fields at the surface of a massive star. MNRAS 356:1139-1148

Najarro F (2001) Spectroscopy of P Cygni. In: de Groot M, Sterken C (eds) P Cygni 2000: 400 Years of Progress, Astronomical Society of the Pacific Conference Series, vol 233, p 133

Najarro F (2006) Metallicity in the GC. Journal of Physics Conference Series 54:224-232

Najarro F (2008) Metallicity studies in the IR: Unveiling Obscured Clusters of our Galaxy. In: Bresolin F, Crowther PA, Puls J (eds) Massive Stars as Cosmic Engines, IAU Symposium, vol 250

Najarro F, Hillier D (2008) The Wind of Eta Carinae and Other LBVs. In: Humphreys R, Davidson K (eds) Eta Carinae

Najarro F, Hillier DJ, Stahl O (1997a) A spectroscopic investigation of P Cygni. I. H and HeI lines. A\&A 326:1117-1134

Najarro F, Krabbe A, Genzel R, Lutz D, Kudritzki RP, Hillier DJ (1997b) Quantitative spectroscopy of the HeI cluster in the Galactic center. A\&A 325:700-708

Najarro F, Kudritzki RP, Hillier DJ, Lamers HJGLM, Voors RHM, Morris PW, Waters LBFM (1998) Ionized Outflows of Hot Stars. Ap\&SS 255:137-144

Najarro F, Figer DF, Hillier DJ, Kudritzki RP (2004) Metallicity in the Galactic Center: The Arches Cluster. ApJL 611:L105-L108

Najarro F, Puls J, Herrero A, Hanson MM, Martin-Pintado J, Hillier DJ (2008) Tracking the Clumping in OB Stars from UV to radio. In: Hamann WR, Oskinova LM, Feldmeier A (eds) Clumping in Hot-Star Winds, pp 43-46

Nugis T, Lamers HJGLM (2000) Mass-loss rates of wolf-rayet stars as a function of stellar parameters. A\&A 360:227-244

Nugis T, Lamers HJGLM (2002) The mass-loss rates of wolf-rayet stars explained by optically thick radiation driven wind models. A\&A 389:162-179 
Nugis T, Crowther P, Willis A (1998) Clumping-corrected mass-loss rates of Wolf-Rayet stars. A\&A 333:956-969

Oskinova L, Ignace R, Hamann WR, Pollock A, Brown J (2003) The conspicuous absence of X-ray emission from carbon-enriched Wolf-Rayet stars. A\&A 402:755-765

Oskinova LM, Feldmeier A, Hamann WR (2004) X-ray emission lines from inhomogeneous stellar winds. A\&A 422:675-691

Oskinova LM, Feldmeier A, Hamann WR (2006) High-resolution X-ray spectroscopy of bright O-type stars. MNRAS 372:313-326

Oskinova LM, Hamann WR, Feldmeier A (2007) Neglecting the porosity of hot-star winds can lead to underestimating mass-loss rates. A\&A 476:1331-1340

Oskinova LM, Hamann WR, Feldmeier A (2008) X-raying clumped stellar winds. In: Hamann WR, Oskinova LM, Feldmeier A (eds) Clumping in Hot-Star Winds, pp 203-208

Osterbrock D, Flather E (1959) Electron Densities in the Orion NEBULA.II. ApJ 129:26

Owocki S, Townsend R, ud-Doula A (2005) Magnetic Channeling of Radiatively Driven HotStar Winds. In: de Gouveia dal Pino EM, Lugones G, Lazarian A (eds) Magnetic Fields in the Universe: From Laboratory and Stars to Primordial Structures., American Institute of Physics Conference Series, vol 784, pp 239-252

Owocki SP (1994a) The Basic Physics of Hot-Star Winds. In: Balona LA, Henrichs HF, Le Contel JM (eds) Pulsation; Rotation; and Mass Loss in Early-Type Stars, IAU Symposium, vol 162, p 475

Owocki SP (1994b) Theory review: Line-driven instability and other causes of structure and variability in hot-star winds. Ap\&SS 221:3-23

Owocki SP (1994c) Theory review: Line-driven instability and other causes of structure and variability in hot-star winds. Ap\&SS 221:3-23

Owocki SP (1999) Co-Rotating Interaction Regions in 2D Hot-Star Wind Models with LineDriven Instability. In: Wolf B, Stahl O, Fullerton AW (eds) IAU Colloq. 169: Variable and Non-spherical Stellar Winds in Luminous Hot Stars, Lecture Notes in Physics, Berlin Springer Verlag, vol 523, p 294

Owocki SP (2008) Dynamical simulation of the velocity-porosity reduction in observed strength of stellar wind lines. In: Hamann WR, Oskinova LM, Feldmeier A (eds) Clumping in HotStar Winds, pp 121-124

Owocki SP, Cohen DH (1999) A Simple Scaling Analysis of X-Ray Emission and Absorption in Hot-Star Winds. ApJ 520:833-840

Owocki SP, Cohen DH (2001) X-Ray Line Profiles from Parameterized Emission within an Accelerating Stellar Wind. ApJ 559:1108-1116

Owocki SP, Cohen DH (2006) The Effect of Porosity on X-Ray Emission-Line Profiles from Hot-Star Winds. ApJ 648:565-571

Owocki SP, Puls J (1996) Nonlocal Escape-Integral Approximations for the Line Force in Structured Line-driven Stellar Winds. ApJ 462:894

Owocki SP, Puls J (1999) Line-driven Stellar Winds: The Dynamical Role of Diffuse Radiation Gradients and Limitations to the Sobolev Approach. ApJ 510:355-368

Owocki SP, Puls J (2002) Ion Runaway Instability in Low-Density, Line-driven Stellar Winds. ApJ 568:965-978

Owocki SP, Rybicki GB (1984) Instabilities in line-driven stellar winds. I - Dependence on perturbation wavelength. ApJ 284:337-350

Owocki SP, Rybicki GB (1985) Instabilities in line-driven stellar winds. II - Effect of scattering. ApJ 299:265-276

Owocki SP, ud-Doula A (2004) The Effect of Magnetic Field Tilt and Divergence on the Mass Flux and Flow Speed in a Line-driven Stellar Wind. ApJ 600:1004-1015

Owocki SP, Castor JI, Rybicki GB (1988) Time-dependent models of radiatively driven stellar winds. I - Nonlinear evolution of instabilities for a pure absorption model. ApJ 335:914-930

Owocki SP, Cranmer SR, Blondin JM (1994) Two-dimensional hydrodynamical simulations of wind-compressed disks around rapidly rotating B stars. ApJ 424:887-904

Owocki SP, Cranmer SR, Fullerton AW (1995) Periodic Variations in Ultraviolet Spectral Lines of the B0.5 Ib Star HD 64760: Evidence for Corotating Wind Streams Rooted in Surface Variations. ApJL 453:L37

Owocki SP, Cranmer SR, Gayley KG (1996) Inhibition FO Wind Compressed Disk Formation by Nonradial Line-Forces in Rotating Hot-Star Winds. ApJL 472:L115

Owocki SP, Cranmer SR, Gayley KG (1998a) Latitudinal Dependence of Radiatively Driven 
Mass Loss from Rapidly Rotating Hot-Stars. In: Hubert AM, Jaschek C (eds) B[e] stars, Astrophysics and Space Science Library, vol 233, p 205

Owocki SP, Gayley KG, Cranmer SR (1998b) Effects of Gravity Darkening on Radiatively Driven Mass Loss from Rapidly Rotating Stars. In: Howarth I (ed) Properties of Hot Luminous Stars, Astronomical Society of the Pacific Conference Series, vol 131, p 237

Owocki SP, Gayley KG, Shaviv NJ (2004) A Porosity-Length Formalism for Photon-Tiringlimited Mass Loss from Stars above the Eddington Limit. ApJ 616:525-541

Panagia N, Felli M (1975) The spectrum of the free-free radiation from extended envelopes. A\&A 39:1-5

Pauldrach A (1987) Radiation driven winds of hot luminous stars. III - Detailed statistical equilibrium calculations for hydrogen to zinc. A\&A 183:295-313

Pauldrach A, Puls J, Kudritzki RP (1986) Radiation-driven winds of hot luminous stars - Improvements of the theory and first results. A\&A 164:86-100

Pauldrach AWA (2005) Radiation Driven Atmospheres of O-type Stars - Synthetic UV-spectra of Consistent Atmospheric Models as a Spectroscopic Tool. In: Szczerba R, Stasińska G, Gorny SK (eds) Planetary Nebulae as Astronomical Tools, American Institute of Physics Conference Series, vol 804, pp 105-116

Pauldrach AWA, Puls J (1990) Radiation-driven winds of hot stars. VIII - The bistable wind of the luminous blue variable P Cygni (B1 Ia/+/). A\&A 237:409-424

Pauldrach AWA, Kudritzki RP, Puls J, Butler K (1990) Radiation driven winds of hot luminous stars. VII - The evolution of massive stars and the morphology of stellar wind spectra. A\&A 228:125-154

Pauldrach AWA, Kudritzki RP, Puls J, Butler K, Hunsinger J (1994) Radiation-driven winds of hot luminous stars. 12: A first step towards detailed UV-line diagnostics of O-stars. A\&A 283:525-560

Pauldrach AWA, Lennon M, Hoffmann TL, Sellmaier F, Kudritzki RP, Puls J (1998) Realistic Models for Expanding Atmospheres. In: Howarth I (ed) Properties of Hot Luminous Stars, Astronomical Society of the Pacific Conference Series, vol 131, p 258

Pauldrach AWA, Hoffmann TL, Lennon M (2001) Radiation-driven winds of hot luminous stars. XIII. A description of NLTE line blocking and blanketing towards realistic models for expanding atmospheres. A\&A 375:161-195

Pauldrach AWA, Hoffmann TL, Méndez RH (2004) Radiation-driven winds of hot luminous stars. XV. Constraints on the mass-luminosity relation of central stars of planetary nebulae. A\&A 419:1111-1122

Pellerin A, Fullerton AW, Robert C, Howk JC, Hutchings JB, Walborn NR, Bianchi L, Crowther PA, Sonneborn G (2002) An Atlas of Galactic OB Spectra Observed with the Far Ultraviolet Spectroscopic Explorer. ApJS 143:159-200

Pelupessy F, Lamers H, Vink J (2000) The radiation driven winds of rotating B[e] supergiants. A\&A 359:695-706

Petit V, Wade GA, Drissen L, Montmerle T, Alecian E (2008) Discovery of two magnetic massive stars in the Orion Nebula Cluster: a clue to the origin of neutron star magnetic fields? MNRAS 387:L23-L27

Petrenz P (1999) Self-consistent models of radiation driven winds of hot stars with rotation (in German). PhD thesis, Ludwig-Maximilians-Universität München

Petrenz P, Puls J (1996) $\mathrm{H} \alpha$ line formation in hot star winds: the influence of rotation. A\&A 312:195-220

Petrenz P, Puls J (2000) 2-D non-LTE models of radiation driven winds from rotating early-type stars. I. Winds with an optically thin continuum. A\&A 358:956-992

Pittard JM, Dougherty SM, Coker RF, O'Connor E, Bolingbroke NJ (2006) Radio emission models of colliding-wind binary systems. Inclusion of IC cooling. A\&A 446:1001-1019

Poe CH, Owocki SP, Castor JI (1990) The steady state solutions of radiatively driven stellar winds for a non-Sobolev, pure absorption model. ApJ 358:199-213

Pollock AMT (1989) Speculation of the origin of X-ray emission in early-type stars. In: Reconnection in Space Plasma, pp 309-311

Prinja BK (1992) UV P Cygni Profile Variability in 0 Stars (Invited Paper). In: Drissen L, Leitherer C, Nota A (eds) Nonisotropic and Variable Outflows from Stars, Astronomical Society of the Pacific Conference Series, vol 22, p 167

Prinja RK (1988) Evidence for rotationally modulated variability in O star winds. MNRAS 231:21P-24P 
Prinja RK (1994) Time-Dependent Phenomena in OB Star Winds. In: Balona LA, Henrichs HF, Le Contel JM (eds) Pulsation; Rotation; and Mass Loss in Early-Type Stars, IAU Symposium, vol 162, p 507

Prinja RK, Smith LJ (1992) Migrating optical depth enhancements in the UV wind lines of the Wolf-Rayet star HD 93131. A\&A 266:377-384

Prinja RK, Barlow MJ, Howarth ID (1990) Terminal velocities for a large sample of O stars, B supergiants, and Wolf-Rayet stars. ApJ 361:607-620

Prinja RK, Balona LA, Bolton CT, Crowe RA, Fieldus MS, Fullerton AW, Gies DR, Howarth ID, McDavid D, Reid AHN (1992) Time series observations of O stars. I - IUE observations of variability in the stellar wind of Zeta Puppis. ApJ 390:266-272

Prinja RK, Massa D, Fullerton AW (1995) The IUE MEGA Campaign: Modulated Structure in the Wind of HD 64760 (B0.5 Ib). ApJL 452:L61

Przybilla N, Butler K, Becker SR, Kudritzki RP (2006) Quantitative spectroscopy of BA-type supergiants. A\&A 445:1099-1126

Puls J (1987) Radiation-driven winds of hot luminous stars. IV - The influence of multi-line effects. A\&A 184:227-248

Puls J (2008) Physical and Wind Properties of OB-Stars. In: Bresolin F, Crowther PA, Puls J (eds) Massive Stars as Cosmic Engines, IAU Symposium, vol 250

Puls J, Owocki SP, Fullerton AW (1993a) On the synthesis of resonance lines in dynamical models of structured hot-star winds. A\&A 279:457-476

Puls J, Pauldrach AWA, Kudritzki RP, Owocki SP, Najarro F (1993b) Radiation Driven Winds of Hot Stars - some Remarks on Stationary Models and Spectrum Synthesis in TimeDependent Simulations. (Ludwig Biermann Award Lecture 1992). In: Klare G (ed) Reviews in Modern Astronomy, Reviews in Modern Astronomy, vol 6, pp 271-300

Puls J, Kudritzki RP, Herrero A, Pauldrach AWA, Haser SM, Lennon DJ, Gabler R, Voels SA, Vilchez JM, Wachter S, Feldmeier A (1996) O-star mass-loss and wind momentum rates in the Galaxy and the Magellanic Clouds Observations and theoretical predictions. A\&A 305:171

Puls J, Kudritzki RP, Santolaya-Rey AE, Herrero A, Owocki SP, McCarthy JK (1998) Spectral Diagnostics of Blue Stars with Winds. In: Howarth I (ed) Properties of Hot Luminous Stars, Astronomical Society of the Pacific Conference Series, vol 131, p 245

Puls J, Petrenz P, Owocki SP (1999) Non-spherical Radiation-Driven Wind Models. In: Wolf B, Stahl O, Fullerton AW (eds) IAU Colloq. 169: Variable and Non-spherical Stellar Winds in Luminous Hot Stars, Lecture Notes in Physics, Berlin Springer Verlag, vol 523, p 131

Puls J, Springmann U, Lennon M (2000) Radiation driven winds of hot luminous stars. XIV. Line statistics and radiative driving. A\&AS 141:23-64

Puls J, Repolust T, Hoffmann TL, Jokuthy A, Venero ROJ (2003) Advances in radiatively driven wind models. In: van der Hucht K, Herrero A, Esteban C (eds) A Massive Star Odyssey: From Main Sequence to Supernova, IAU Symposium, vol 212, p 61

Puls J, Urbaneja MA, Venero R, Repolust T, Springmann U, Jokuthy A, Mokiem MR (2005) Atmospheric NLTE-models for the spectroscopic analysis of blue stars with winds. II. Lineblanketed models. A\&A 435:669-698

Puls J, Markova N, Scuderi S, Stanghellini C, Taranova OG, Burnley AW, Howarth ID (2006) Bright OB stars in the Galaxy. III. Constraints on the radial stratification of the clumping factor in hot star winds from a combined $\mathrm{H}_{\alpha}$, IR and radio analysis. A\&A 454:625-651

Reimer A, Pohl M, Reimer O (2006) Nonthermal High-Energy Emission from Colliding Winds of Massive Stars. ApJ 644:1118-1144

Repolust T, Puls J, Herrero A (2004) Stellar and wind parameters of Galactic O-stars. The influence of line-blocking/blanketing. A\&A 415:349-376

Repolust T, Puls J, Hanson MM, Kudritzki RP, Mokiem MR (2005) Quantitative H and K band spectroscopy of Galactic OB-stars at medium resolution. A\&A 440:261-286

Robert C (1994) Optical spectroscopy of inhomogeneities in the winds of Wolf-Rayet stars. Ap\&SS 221:137-153

Robert C, Moffat A, Bastien P, Drissen L, St-Louis N (1989) Polarization variability among Wolf-Rayet stars. V - Linear polarization of the bright Cygnus stars and an anticorrelation of variability with wind speed. ApJ 347:1034-1054

Rodrigues C, Magalhaes A (2000) Blobs in Wolf-Rayet Winds: Random Photometric and Polarimetric Variability. ApJ 540:412-421

Runacres MC, Owocki SP (2002) The outer evolution of instability-generated structure in radia- 
tively driven stellar winds. A\&A 381:1015-1025

Runacres MC, Owocki SP (2005) A pseudo-planar, periodic-box formalism for modelling the outer evolution of structure in spherically expanding stellar winds. A\&A 429:323-333

Rybicki GB, Owocki SP, Castor JI (1990) Instabilities in line-driven stellar winds. IV - Linear perturbations in three dimensions. ApJ 349:274-285

Sana H, Rauw G, Naze Y, Gosset E, Vreux JM (2006) An XMM-Newton view of the young open cluster NGC 6231 - II. The OB star population. MNRAS 372:661-678

Santolaya-Rey AE, Puls J, Herrero A (1997) Atmospheric NLTE-models for the spectroscopic analysis of luminous blue stars with winds. A\&A 323:488-512

Schmutz W (1995) Hydrodynamnic line-blanketed atmospheres of Wolf-Rayet stars (Invited). In: van der Hucht KA, Williams PM (eds) Wolf-Rayet Stars: Binaries; Colliding Winds; Evolution, IAU Symposium, vol 163, p 127

Schmutz W (1997) Photon loss from the helium Ly $\alpha$ line - the key to the acceleration of WolfRayet winds. A\&A 321:268-287

Schnerr R (2007) Magnetic fields and mass loss in massive stars. PhD thesis, University of Amsterdam

Scuderi S, Bonanno G, di Benedetto R, Spadaro D, Panagia N (1992) H-alpha observations of early-type stars. ApJ 392:201-208

Semel M (1989) Zeeman-Doppler imaging of active stars. I - Basic principles. A\&A 225:456466

Shaviv NJ (1998) The Eddington Luminosity Limit for Multiphased Media. ApJL 494:L193

Shaviv NJ (2001) The theory of steady-state super-Eddington winds and its application to novae. MNRAS 326:126-146

Sim S (2004) Mass-loss rates for hot luminous stars: the influence of line branching. MNRAS 349:899-908

Smith LJ, Crowther PA, Prinja RK (1994) A study of the luminous blue variable candidate He 3-519 and its surrounding nebula. A\&A 281:833-854

Smith N, Owocki SP (2006) On the Role of Continuum-driven Eruptions in the Evolution of Very Massive Stars and Population III Stars. ApJL 645:L45-L48

Smith N, Davidson K, Gull T, Ishibashi K, Hillier D (2003a) Latitude-dependent effects in the stellar wind of eta Carinae. ApJ 586:432-450

Smith N, Gehrz RD, Hinz PM, Hoffmann WF, Hora JL, Mamajek EE, Meyer MR (2003b) Mass and Kinetic Energy of the Homunculus Nebula around $\eta$ Carinae. AJ 125:1458-1466

Smith N, Vink JS, de Koter A (2004) The Missing Luminous Blue Variables and the Bistability Jump. ApJ 615:475-484

Sobolev VV (1960) Moving envelopes of stars. Cambridge: Harvard University Press, 1960

Springmann U (1991) Diploma-Thesis. Kinetic effects in hot star winds (in German). LudwigMaximilians-Universität München

Springmann U (1994) Multiple resonance line scattering and the 'momentum problem' in WolfRayet star winds. A\&A 289:505-523

Springmann U, Puls J (1998) Models of Radiatively Driven Wolf-Rayet-Star Winds. In: Howarth I (ed) Properties of Hot Luminous Stars, Astronomical Society of the Pacific Conference Series, vol 131, p 286

Springmann UWE, Pauldrach AWA (1992) Radiation-driven winds of hot luminous stars. XI Frictional heating in a multicomponent stellar wind plasma and decoupling of radiatively accelerated ions. A\&A 262:515-522

Spruit HC (1999) Differential rotation and magnetic fields in stellar interiors. A\&A 349:189202

Spruit HC (2002) Dynamo action by differential rotation in a stably stratified stellar interior. A\&A 381:923-932

St-Louis N, Moffat AFJ (2008) Do clumping corrections increase with decreasing mass-loss rate? In: Hamann WR, Oskinova LM, Feldmeier A (eds) Clumping in Hot-Star Winds, pp $39-42$

St-Louis N, Drissen L, Moffat AFJ, Bastien P, Tapia S (1987) Polarization variability among Wolf-Rayet stars. I. Linear polarization of a complete sample of southern Galactic WC stars. ApJ 322:870-887

St-Louis N, Moffat A, Lapointe L, Efimov Y, Shakhovskoj N, Fox G, Piirola V (1993) Polarization eclipse model of the Wolf-Rayet binary V444 Cygni with constraints on the stellar radii and an estimate of the Wolf-Rayet mass-loss rate. ApJ 410:342-356 
Stahl O, Wolf B, Aab O, Smolinski J (1991) Stellar wind properties of A-type hypergiants. A\&A 252:693-700

Stahl O, Jankovics I, Kovacs J, Wolf B, Schmutz W, Kaufer A, Rivinius T, Szeifert T (2001) Long-term spectroscopic monitoring of the Luminous Blue Variable AG Carinae. A\&A 375:54-69

Stevens IR (1995) Thermal radio emission from early-type binary systems. MNRAS 277:163172

Taresch G, Kudritzki RP, Hurwitz M, Bowyer S, Pauldrach AWA, Puls J, Butler K, Lennon DJ, Haser SM (1997) Quantitative analysis of the FUV, UV and optical spectrum of the O3 star HD 93129A. A\&A 321:531-548

Taylor M, Nordsieck KH, Schulte-Ladbeck RE, Bjorkman KS (1991) A study of the asymmetric wind of P Cygni. AJ 102:1197-1206

Townsend RHD, Owocki SP (2005) A rigidly rotating magnetosphere model for circumstellar emission from magnetic OB stars. MNRAS 357:251-264

Townsend RHD, Owocki SP, Groote D (2005) The Rigidly Rotating Magnetosphere of $\sigma$ Orionis E. ApJL 630:L81-L84

Townsend RHD, Owocki SP, ud-Doula A (2007) A Rigid-Field Hydrodynamics approach to modelling the magnetospheres of massive stars. MNRAS 382:139-157

Trundle C, Lennon D (2005) Understanding B-type supergiants in the low metallicity environment of the SMC II. A\&A 434:677-689

Trundle C, Dufton PL, Hunter I, Evans CJ, Lennon DJ, Smartt SJ, Ryans RSI (2007) The VLTFLAMES survey of massive stars: evolution of surface $\mathrm{N}$ abundances and effective temperature scales in the Galaxy and Magellanic Clouds. A\&A 471:625-643

Trundle C, Kotak R, Vink JS, Meikle WPS (2008) SN 2005 gj: Evidence for LBV supernovae progenitors? A\&A 483:L47-L50

ud-Doula A, Owocki SP (2002) Dynamical Simulations of Magnetically Channeled Line-driven Stellar Winds. I. Isothermal, Nonrotating, Radially Driven Flow. ApJ 576:413-428

ud-Doula A, Townsend RHD, Owocki SP (2006) Centrifugal Breakout of Magnetically Confined Line-driven Stellar Winds. ApJL 640:L191-L194

ud-Doula A, Owocki SP, Townsend RHD (2008) Dynamical simulations of magnetically channelled line-driven stellar winds - II. The effects of field-aligned rotation. MNRAS 385:97108

Urbaneja M (2004) B Supergiants in the Milky Way and Nearby Galaxies: Models and Quantitative Spectroscopy. PhD thesis, Universidad de la Laguna, La Laguna, Spain

Urbaneja MA, Kudritzki R, Bresolin F, Przybilla N, Gieren W, Pietrzynski G (2008) The Araucaria Project: the Local Group Galaxy WLM-Distance and metallicity from quantitative spectroscopy of blue Supergiants. ArXiv e-prints 805,0805.3555

Vacca WD, Garmany CD, Shull JM (1996) The Lyman-Continuum Fluxes and Stellar Parameters of O and Early B-Type Stars. ApJ 460:914

van Boekel R, Kervella P, Scholler M, Herbst T, Brandner W, de Koter A, Waters LF, Hillier D, Paresce F, Lenzen R, Lagrange AM (2003) Direct measurement of the size and shape of the present-day stellar wind of eta Carinae. A\&A 410:L37-L40

Van Loo S (2005) Non-thermal radio emission from single hit stars. PhD thesis, AA(Royal Observatory of Belgium Ringlaan 3 B-1180 Brussels)

van Loo S, Runacres MC, Blomme R (2006) Can single O stars produce non-thermal radio emission? A\&A 452:1011-1019

van Marle AJ, Owocki SP, Shaviv NJ (2008) Continuum-Driven Winds from Super-Eddington Stars: A Tale of Two Limits. In: First Stars III, American Institute of Physics Conference Series, vol 990, pp 250-253

Verdugo E, Talavera A, Gómez de Castro AI, Henrichs HF (2003) Search for magnetic fields in A-type supergiants. In: van der Hucht K, Herrero A, Esteban C (eds) A Massive Star Odyssey: From Main Sequence to Supernova, IAU Symposium, vol 212, p 255

Villamariz M (2001) . PhD thesis, Universidad de la Laguna, La Laguna, Spain

Vink JS (2006) Massive star feedback - from the first stars to the present. In: Lamers HJGLM, Langer N, Nugis T, Annuk K (eds) ASP Conf. Ser. 353: Stellar Evolution at Low Metallicity: Mass Loss, Explosions, Cosmology, p 113

Vink JS (2007) Constraining GRB progenitor models by probing Wolf-Rayet wind geometries in the Large Magellanic Cloud. A\&A 469:707-711

Vink JS (2009) Eta Carinae and the Luminous Blue Variables. In: Humphreys R, Davidson K 
(eds) Eta Carinae and the supernova imposters

Vink JS, Cassisi S (2002) Hot horizontal branch stars: Predictions for mass loss. Winds, rotation, and the low gravity problem. A\&A 392:553-562

Vink JS, de Koter A (2002) Predictions of variable mass loss for Luminous Blue Variables. A\&A 393:543-553

Vink JS, de Koter A (2005) On the metallicity dependence of Wolf-Rayet winds. A\&A 442:587596

Vink JS, de Koter A, Lamers HJGLM (1999) On the nature of the bi-stability jump in the winds of early-type supergiants. A\&A 350:181-196

Vink JS, de Koter A, Lamers HJGLM (2000) New theoretical mass-loss rates of O and B stars. A\&A 362:295-309

Vink JS, de Koter A, Lamers HJGLM (2001) Mass-loss predictions for O and B stars as a function of metallicity. A\&A 369:574-588

von Zeipel H (1924) The radiative equilibrium of a rotating system of gaseous masses. MNRAS 84:665-683

Walborn NR, Fullerton AW, Crowther PA, Bianchi L, Hutchings JB, Pellerin A, Sonneborn G, Willis AJ (2002) Far Ultraviolet Spectroscopic Explorer Atlas of OB Stars in the Magellanic Clouds. ApJS 141:443-468

Waldron WL, Cassinelli JP (2007) An Extensive Collection of Stellar Wind X-Ray Source Region Emission Line Parameters, Temperatures, Velocities, and Their Radial Distributions as Obtained from Chandra Observations of 17 OB Stars. ApJ 668:456-480

Waldron WL, Cassinelli JP (2001) Chandra Discovers a Very High Density X-Ray Plasma on the O Star $\zeta$ Orionis. ApJL 548:L45-L48

Waters LBFM, Lamers HJGLM (1984) The infrared and radio spectrum of early type stars with mass loss. II - Tables of theoretical curves of growth for IR and radio excess and gaunt factors. A\&AS 57:327-352

Weigelt G, Kraus S, Driebe T, Petrov RG, Hofmann KH, Millour F, Chesneau O, Schertl D, Malbet F, Hillier JD, and 93 coauthors (2007) Near-infrared interferometry of $\eta$ Carinae with spectral resolutions of 1500 and 12000 using AMBER/VLTI. A\&A 464:87-106

Wende S, Glatzel W, Schuh S (2008) Non-linear Pulsations in Wolf-Rayet Stars. In: Werner A, Rauch T (eds) Hydrogen-Deficient Stars, Astronomical Society of the Pacific Conference Series, vol 391, p 319

White RL (1985) Synchrotron emission from chaotic stellar winds. ApJ 289:698-708

White RL, Becker RH (1983) The discovery of a hot stellar wind. ApJL 272:L19-L23

Williams PM, van der Hucht KA, The PS (1987) Infrared photometry of late-type Wolf-Rayet stars. A\&A 182:91-106

Williams PM, van der Hucht KA, Pollock AMT, Florkowski DR, van der Woerd H, Wamsteker WM (1990) Multi-frequency variations of the Wolf-Rayet system HD 193793. I - Infrared, X-ray and radio observations. MNRAS 243:662-684

Williams PM, Dougherty SM, Davis RJ, van der Hucht KA, Bode MF, Setia Gunawan DYA (1997) Radio and infrared structure of the colliding-wind Wolf-Rayet system WR147. MNRAS 289:10-20

Wojdowski PS, Schulz NS (2005) Ion-by-Ion Differential Emission Measure Determination of Collisionally Ionized Plasma. II. Application to Hot Stars. ApJ 627:953-959

Wolf B, Stahl O, Fullerton AW (eds) (1999) Variable and Non-spherical Stellar Winds in Luminous Hot Stars, Lecture Notes in Physics, Berlin Springer Verlag, vol 523

Woosley SE (1993) Gamma-ray bursts from stellar mass accretion disks around black holes. ApJ 405:273-277

Wright AE, Barlow MJ (1975) The radio and infrared spectrum of early-type stars undergoing mass loss. MNRAS 170:41-51

Zahn JP (1992) Circulation and turbulence in rotating stars. A\&A 265:115-132

Zickgraf FJ, Wolf B, Leitherer C, Appenzeller I, Stahl O (1986) B(e)-supergiants of the Magellanic Clouds. A\&A 163:119-134

Zickgraf FJ, Wolf B, Stahl O, Humphreys RM (1989) S 18 - A new B(e) supergiant in the Small Magellanic Cloud with evidence for an excretion disk. A\&A 220:206-214

Zsargó J, Hillier DJ, Bouret JC, Lanz T, Leutenegger MA, Cohen DH (2008a) On the Importance of the Interclump Medium for Superionization: O VI Formation in the Wind of $\zeta$ Puppis. ApJL 685:L149-L152

Zsargó J, Hillier DJ, Georgiev LN (2008b) Axi-symmetric models of B[e] supergiants. I. The ef- 
fective temperature and mass-loss dependence of the hydrogen and helium ionization structure. A\&A 478:543-551 